\title{
HYDROLOGIC DATA FOR THE BIG SPRING BASIN, CLAYTON COUNTY, IOWA, WATER YEAR 1990
}

By S.J. Kalkhoff, R. L. Kuzniar, D.L. Kolpin, and C.A. Harvey

U. S. GEOLOGICAL SURVEY

Open-File Report 92-67

Prepared in cooperation with the IOWA DEPARTMENT OF NATURAL RESOURCES

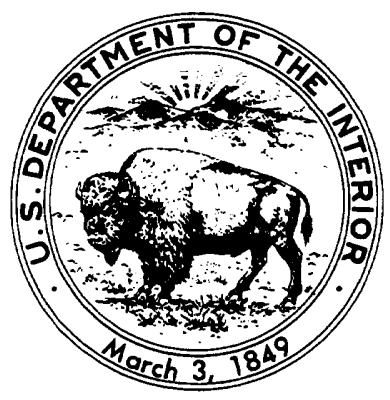
(GEOLOGICAL SURVEY BUREAU) 


\title{
U.S. DEPARTMENT OF THE INTERIOR
}

\author{
MANUEL LUJAN, JR., Secretary
}

\author{
U.S. GEOLOGICAL SURVEY
}

Dallas L. Peck, Director

For additional information write to:

Copies of this report can be purchased from:

District Chief

U.S. Geological Survey

Rm. 269, Federal Building

400 South Clinton Street

Iowa City, Iowa 52244
U.S. Geological Survey

Open-File Reports

Earth Science Information Center

Box 25425

Denver, Colorado 80225 


\section{CONTENTS}

Page

Introduction

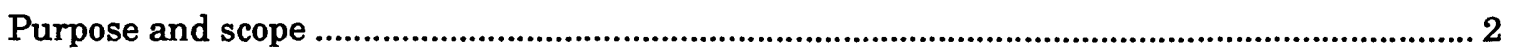

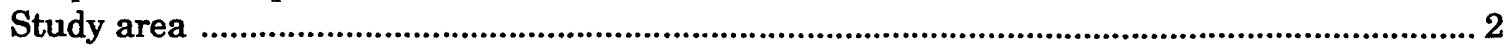

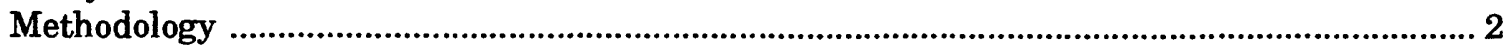

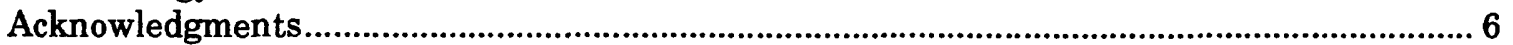

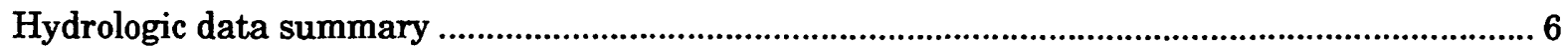

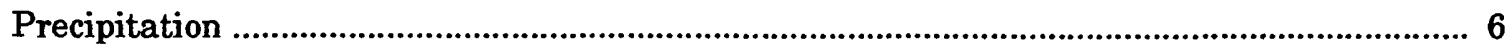

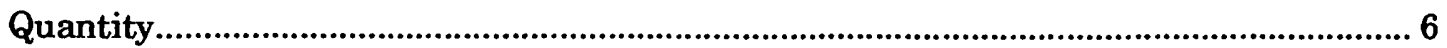

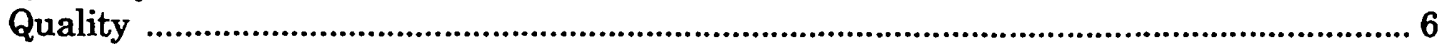

Surface water

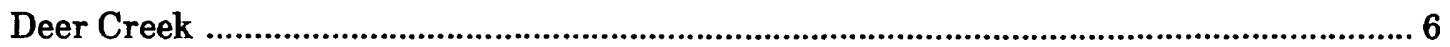

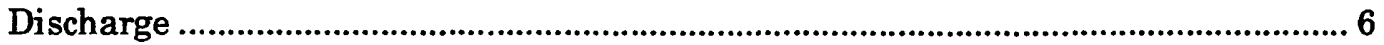

Water quality …………….................................................................................... 7

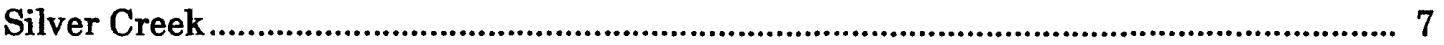

Unnamed Creek ............................................................................................................. 7

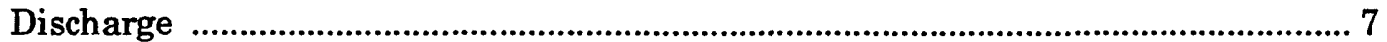

Water quality ................................................................................................. 7

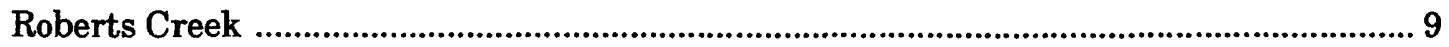

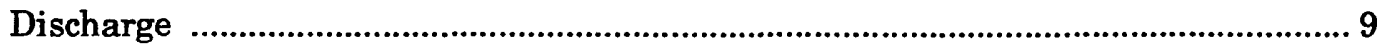

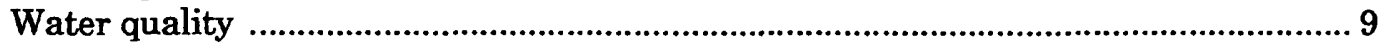

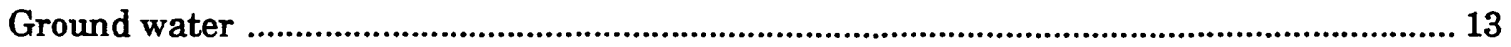

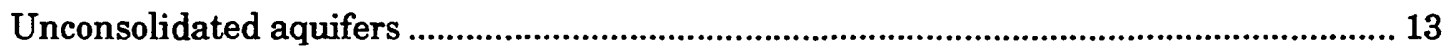

Galena aquifer .................................................................................................. 19

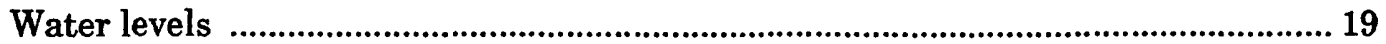

Big Spring ……………............................................................................................. 19

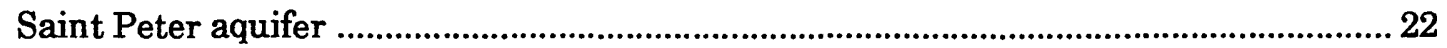

Seepage measurements ........................................................................................................ 22

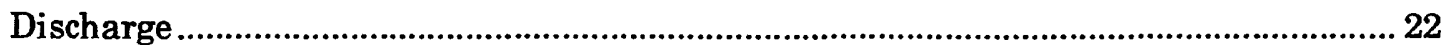

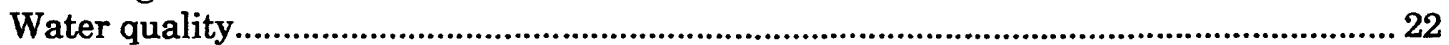

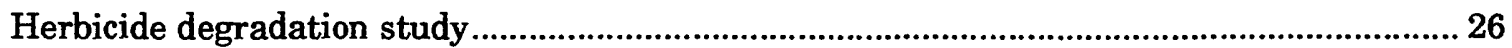

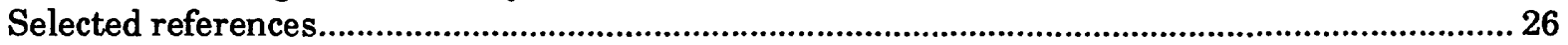

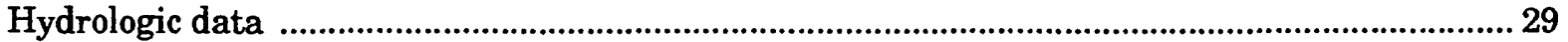




\section{ILLUSTRATIONS}

Figure 1-2. Maps showing:

1. Location of Big Spring study area and rain gages............................................... 3

2. Location of surface- and ground-water-quality monitoring sites ......................... 4

Figure 3-15. Graphs showing:

3. Selected chemical constituents and stream discharge at site DC5, Deer Creek near Postville, Iowa, water year 1990.

4. Relation of deuterium to oxygen-stable isotopes at sites in the Deer Creek watershed, water year 1990

5. Daily mean discharge at site L23S, Silver Creek near Luana, Iowa, water year 1990

6. Flow duration at monitoring sites in the Big Spring basin, Iowa, water year 1990 .

7. Suspended-sediment load and streamflow discharge at three monitoring sites in the Big Spring basin, water year 1990.

8. Daily precipitation, daily mean specific conductance, discharge, water temperature, and daily median $\mathrm{pH}$ at site $\mathrm{RC2}$, Roberts Creek above Saint Olaf, Iowa, water year 1990.

9. Daily mean water levels in unconsolidated aquifers in the Big Spring basin, water year 1990 .

10. Total dissolved-nitrogen concentrations in water from lysimeters and water levels in well DCW1 in the unconsolidated aquifer of the Deer Creek watershed, water year 1990

11. Total-recoverable atrazine concentrations in water from lysimeters and water levels in well DCW1 in the unconsolidated aquifer of the Deer Creek watershed, water year 1990

12. Total-recoverable metolachlor concentrations in water from lysimeters and water levels in well DCW1 in the unconsolidated aquifer of the Deer Creek watershed, water year 1990

13. Daily mean water levels in the Galena aquifer in the Big Spring basin, water year 1990

14. Weekly precipitation, daily mean specific conductance, discharge, water temperature, and daily median $\mathrm{pH}$ at Big Spring, water year 1990.

15. Daily mean water levels in the Saint Peter aquifer in the Big Spring basin, water year 1990

16. Map showing location of seepage-study sampling sites 24 


\section{ILLUSTRATIONS--Continued}

Figure 17. Graph showing stream discharge and total dissolved-nitrogen and totalrecoverable atrazine concentrations and loads in Roberts Creek, May 2930,1990 .

18. Map showing location of degradation-study sampling sites in a selected reach of Roberts Creek

19. Graph showing deethylatrazine plus deisopropylatrazine-to-atrazine ratio in a selected reach of Roberts Creek, April-November 1990

\section{TABLES}

Table 1. Records of monitoring sites in the Big Spring basin, Clayton County, Iowa........ 30

2. Records of selected monitoring wells, lysimeters, and tile line in the Big Spring basin, Clayton County, Iowa

3. Location and drainage area of seepage-study sampling sites, Clayton County, Iowa

4. Sample preparation and analytical methods.

5. Statistical summary of precipitation quantity and quality at Big Spring, water year 1990

6. Accumulated daily precipitation, water year 1990

7. Daily mean discharge at site DC5, Deer Creek near Postville, Iowa, water year 1990

8. Onsite determinations of selected water-quality constituents at surfacewater monitoring sites in the Big Spring basin, Clayton County, Iowa, water year 1990

9. Concentrations of major ions at selected surface-water monitoring sites in the Big Spring basin, Clayton County, Iowa, water year 1990

10. Selected nitrogen, phosphorus, and carbon species at surface-water monitoring sites in the Big Spring basin, Clayton County, Iowa, water year 1990

11. Selected pesticides at surface-water monitoring sites in the Big Spring basin, Clayton County, Iowa, water year 1990.

12. Stable isotopic ratios at sites in the Deer Creek subbasin, Clayton County, Iowa, water year 1990.

13. Daily mean discharge at site L23S, Silver Creek near Luana, Iowa, water year 1990

14. Daily mean discharge at site BOOGD, Unnamed Creek near Luana, Iowa, water year 1990 


\section{TABLES--Continued}

Table 15. Daily mean suspended-sediment concentrations and daily suspendedsediment load at site BOOGD, Unnamed Creek near Luana, Iowa, water year 1990

16. Daily mean discharge and specific conductance at site RC2, Roberts Creek above Saint Olaf, Iowa, water year 1990

17. Daily median pH and mean water temperature at site RC2, Roberts Creek above Saint Olaf, Iowa, water year 1990

18. Daily mean suspended-sediment concentrations and daily suspendedsediment load at site RC2, Roberts Creek above Saint Olaf, Iowa, water year 1990

19. Miscellaneous water-level measurements in the Big Spring basin, Clayton County, Iowa, water year 1990.

20. Daily mean water levels in unconsolidated aquifers, Clayton County, Iowa, water year 1990

21. Onsite measurements of selected water-quality constituents at selected ground-water monitoring sites in the Big Spring basin, Clayton County, Iowa, water year 1990.

22. Concentrations of major ions at selected ground-water monitoring sites in the Big Spring basin, Clayton County, Iowa, water year 1990

23. Selected nitrogen, phosphorus, and carbon species at ground-water monitoring sites in the Big Spring basin, Clayton County, Iowa, water year 1990.

24. Selected pesticides at ground-water monitoring sites in the Big Spring basin, Clayton County, Iowa, water year 1990.

25. Daily mean water levels in the Galena aquifer, Clayton County, Iowa, water year 1990

26. Daily mean discharge and specific conductance at Big Spring, Clayton County, Iowa, water year 1990 .

27. Daily median $\mathrm{pH}$ and daily mean water temperature at Big Spring, Clayton County, Iowa, water year 1990.

28. Daily mean suspended-sediment concentrations and daily suspendedsediment load at Big Spring, Clayton County, Iowa, water year 1990.

29. Daily mean water levels in the Saint Peter aquifer, Clayton County, Iowa, water year 1990

30. Onsite determinations and chemical analyses of samples from streams in the Big Spring basin during low-flow conditions, May 29-30, 1990

31. Selected pesticides in samples from streams in the Big Spring basin during low-flow conditions, May 29-30, 1990 


\section{TABLES--Continued}

Table 32. Onsite determinations, nitrogen species, and suspended sediment in a selected reach of Roberts Creek, Clayton County, Iowa, April-November 1990

33. Herbicide and herbicide metabolite concentrations in a selected reach of Roberts Creek, Clayton County, Iowa, April-November 1990.

34. Herbicide concentrations in a selected reach of Roberts Creek, Clayton County, Iowa, April-November 1990.

\section{CONVERSION FACTORS, ABBREVIATIONS, AND VERTICAL DATUM}

\section{Multiply}

inch (in.)

foot (ft)

mile (mi)

acre

acre

square foot $\left(\mathrm{ft}^{2}\right)$

cubic foot per second $\left(\mathrm{ft}^{3} / \mathrm{s}\right)$

pound (lb)

pound per day (lb/d)

ton, short

ton per day (ton/d)

degree Fahrenheit $\left({ }^{\circ} \mathbf{F}\right)$

$\begin{aligned} 1{ }^{\circ} \mathrm{C} & =5 / 9\left({ }^{\circ} \mathrm{F}-32\right) . \\ { }^{\circ} \mathrm{F} & =9 / 5\left({ }^{\circ} \mathrm{C}\right)+32 .\end{aligned}$
By

\section{To obtain}

\section{Length}

25.4

0.3048

1.609

\section{Area}

4,047

0.4047

929.0

Flow

0.02832

cubic meter per second

Mass

0.454

0.454

0.907

0.907

square meter

hectare

square centimeter

millimeter

meter

kilometer

\section{Temperature}

kilogram
kilogram per day
megagram
megagram per day

degree Celsius $\left({ }^{\circ} \mathrm{C}\right)$

Sea level: In this report, "sea level" refers to the National Geodetic Vertical Datum of 1929--a geodetic datum derived from a general adjustment of the first order level nets of the United States and Canada, formerly called Sea Level Datum of 1929.

Water year: A water year is a 12-month period, from October 1 through September 30, designated by the calendar year in which it ends. Years are water years in this report unless otherwise stated. 


\title{
HYDROLOGIC DATA FOR THE BIG SPRING BASIN, CLAYTON COUNTY, IOWA, WATER YEAR 1990
}

\author{
By S.J. Kalkhoff, R.L. Kuzniar, D.W. Kolpin, and C.A. Harvey
}

\section{ABSTRACT}

Hydrologic data were collected in the Big Spring basin located in Clayton County, lowa, during the 1990 water year. The data were collected by the U.S. Geological Survey in cooperation with the lowa Department of Natural Resources, Geological Survey Bureau, to provide information on variation and movement of agricultural chemicals in the hydrologic cycle in the basin. Precipitation, surface-water, and ground-water data were collected.

Rainfall recorded during water year 1990 at a monitoring site on Roberts Creek totaled 43.67 inches. The greatest monthly rainfall (14.45 inches) occurred in August. Calcium and sulfate were the predominant ions in the rain, and the median concentrations of nitrate and ammonia as nitrogen were 0.35 and 0.48 milligrams per liter, respectively.

Stream discharge, specific conductance, $\mathrm{pH}$, and water temperature were monitored continuously, and monthly water-quality samples were collected at three sites in the basin. The predominant ions in samples from Roberts Creek at the point where it leaves the basin were calcium, magnesium, and bicarbonate. Nitrite plus nitrate as nitrogen concentrations in $\mathbf{4 2}$ samples ranged from less than 0.10 to 18 milligrams per liter. Pesticide concentrations in 34 samples ranged from less than 0.10 to 12 micrograms per liter. Alachlor was detected in 56 percent of the samples; atrazine in 100 percent; cyanazine in 68 percent; and metolachlor in 47 percent.

At Big Spring, the ground-water discharge point, the daily mean specific conductance ranged from 378 to 796 microsiemens per centimeter at 25 degrees Celsius, the daily median $\mathrm{pH}$ ranged from 6.5 to 7.2 , and the daily mean water temperature ranged from 5.5 to 11.5 degrees Celsius. Calcium, magnesium, and bicarbonate generally were the predominant ions in solution. Concentrations of nitrite plus nitrate as nitrogen in 32 samples ranged from 3.4 to 16 milligrams per liter. Alachlor was detected in 22 percent of the samples; atrazine in 100 percent; cyanazine in 44 percent, and metolachlor in 6 percent. The maximum atrazine concentration was 4.5 micrograms per liter.

During a low-flow seepage study, May 29 and 30,1990 , the measured discharge lost by streams in the basin was 8.56 cubic feet per second, the measured dissolved nitrogen load lost was 0.29 ton per day, and the measured atrazine load lost was 0.028 pound per day. The total measured discharge and total dissolved nitrogen load leaving the basin in streams were 3.63 cubic feet per second and about 0.04 ton per day, respectively.

\section{INTRODUCTION}

There is interest nationally, as well as within the State of Iowa, to understand, quantify, and minimize the occurrence of agricultural chemicals in surface and ground water. In response to this interest, the Big Spring ground-water basin in Clayton County, Iowa, has been studied since 1980 and has become a nationally known demonstration area for improving ground-water quality through the modification of agricultural practices. Numerous multidisciplinary studies that deal with agronomy, geology, hydrology, biology, and socioeconomics of the basin currently are being conducted.

The unique ground-water-flow system in the Big Spring basin aids in studying the movement of agricultural chemicals in ground water. Much of the ground water in the basin is intercepted by a karst system within the Galena aquifer and is discharged at Big Spring. The extent of the ground-water drainage basin has been defined by dye tracing, potentiometric-surface mapping, and other hydrologic analyses (Hallberg and others, 1983). Nearly all land in the basin is farmed, and a clear link between agricultural chemicals and ground-water contamination has been established (Hallberg and others, 1983, 1984; Libra and others, 1986). 
Beginning in October 1987, the U.S. Geological Survey, in cooperation with the Iowa Department of Natural Resources, Geological Survey Bureau, collected water quantity and quality data in the Big Spring basin. These data are needed to refine the understanding of the hydrologic cycle in the basin and the agricultural-chemical transport processes in the surface- and ground-water systems. The dynamic nature of surface- and ground-water flow in the basin requires that some facets of water quantity and quality be monitored continuously. The data collected in this study aids in the understanding of the flow system.

This report is the third in a series of data reports that present the data collected by the U.S. Geological Survey in the Big Spring basin. Previous reports document data collected by the U.S. Geological Survey in water years 1988 (Kalkhoff, 1989) and 1989 (Kalkhoff and Kuzniar, 1991)

\section{Purpose and Scope}

The purpose of this report is to present the hydrologic data collected in the Big Spring basin by the U.S. Geological Survey during water year 1990. These data include information on the quantity and quality of precipitation and surface and ground water. The scope of data-collection activities includes measuring the input (precipitation) and the output (stream and spring discharge) from the system. Also included is continuous monitoring of selected water-quality constituents (specific conductance, $\mathrm{pH}$, and temperature) of the water leaving the hydrologic system. In addition, suspended-sediment load leaving the basin is determined.

In support of three studies to define the processes affecting surface-water quality, discharge measurements were made at numerous sites on streams in the Big Spring basin to define areas where seepage from surface water was contributing to the ground-water flow system. Water samples were collected for the analysis of nutrients and selected herbicides immediately after each discharge measurement. Hydrologic data collected in the Deer Creek subbasin to study surface- and ground-water relations in a small drainage basin also are presented in this report along with the results of analyses of water sampled in a losing reach of Roberts Creek to quantify the degradation of agricultural chemicals.

Hydrologic data are summarized and presented graphically in the text and in tables in the "Hydrologic Data" section in the back of the report. Additional water-discharge and chemical data, which are not included in this report, are collected by State, Federal, and university researchers through ongoing studies. A report detailing hydrogeologic observations from bedrock monitoring wells in the Big Spring basin was released in water year 1990 by the Iowa Department of Natural Resources, Geological Survey Bureau (Rowden and Libra, 1990).

\section{Study Area}

The study area (fig. 1), located in Clayton County in northeastern Iowa, corresponds to the 103-mi ${ }^{2}$ ground-water basin that drains through Big Spring (Hallberg and others, 1983). Streams in the study area include Roberts Creek and its major tributary, Silver Creek, which drain approximately 69 percent $\left(70.7 \mathrm{mi}^{2}\right)$ of the area within the ground-water basin. The remaining area is drained by Howard Creek (approximately $18 \mathrm{mi}^{2}$ ), and Hatchery Creek $\left(8.8 \mathrm{mi}^{2}\right)$, and several small intermittent streams. The geology and aquifers in the Big Spring basin are described in detail by Hallberg and others (1983) and will be briefly summarized here. Unconsolidated aquifers are generally found throughout the basin in the loess and alluvial deposits. The Galena aquifer and the Saint Peter aquifer are in bedrock material underlying the entire basin.

\section{Methodology}

Precipitation was measured at three sites (fig. 1). At two sites, RC2 and BOOGD, precipitation was recorded digitally every 15 minutes. At Big Spring, precipitation was recorded continuously.

Precipitation samples were collected automatically at Big Spring with a wet/dry precipitation collector. During periods of precipitation, a container was exposed to catch the rain or snow. Between rains the container 


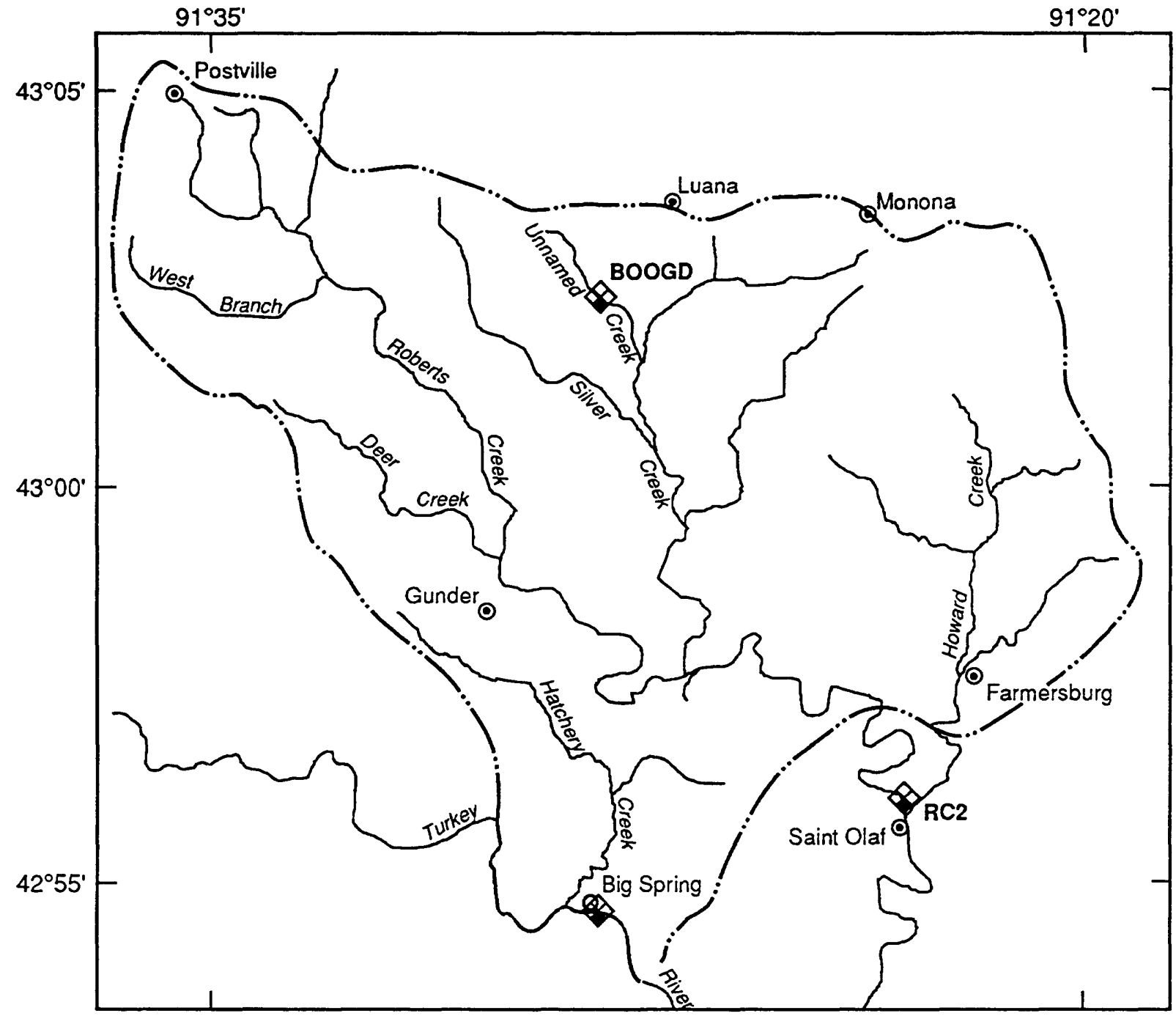

Base from U.S. Geological Survey

Clayton County, 1:100,000,1985

Big Spring ground-water basin location from Hallberg and others, 1983

\section{EXPLANATION}

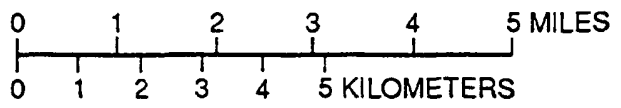

-.. BIG SPRING GROUND-WATER BASIN DIVIDE

9 SPRING

RAIN GAGE AND SITE IDENTIFICATION

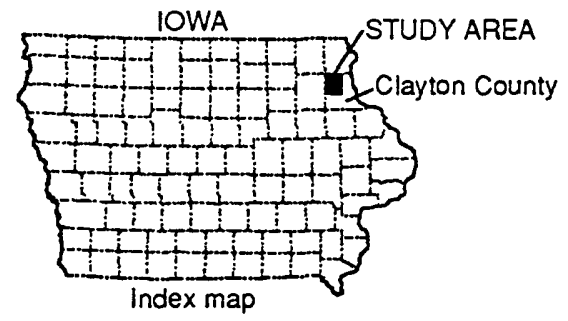

Figure 1. Location of Big Spring study area and rain gages. 
was covered to avoid the collection of particulate matter. Buckets were removed, and the contents analyzed weekly. Site operations are described by the National Atmospheric Deposition Program/National Trends Network (1988).

Water quality was monitored continuously in Roberts Creek above Saint Olaf (site RC2), the primary surface-water discharge point, and at Big Spring, the primary ground-water discharge point (fig. 2). Water samples for chemical analyses were collected monthly at these two sites, Unnamed Creek near Luana (site BOOGD), and Deer Creek near Postville (site DC5). Stream and spring stage also were recorded continuously for later calculation of discharge. Additional samples were collected during periods of snowmelt and intense rainfall. Descriptions of the water-quality monitoring

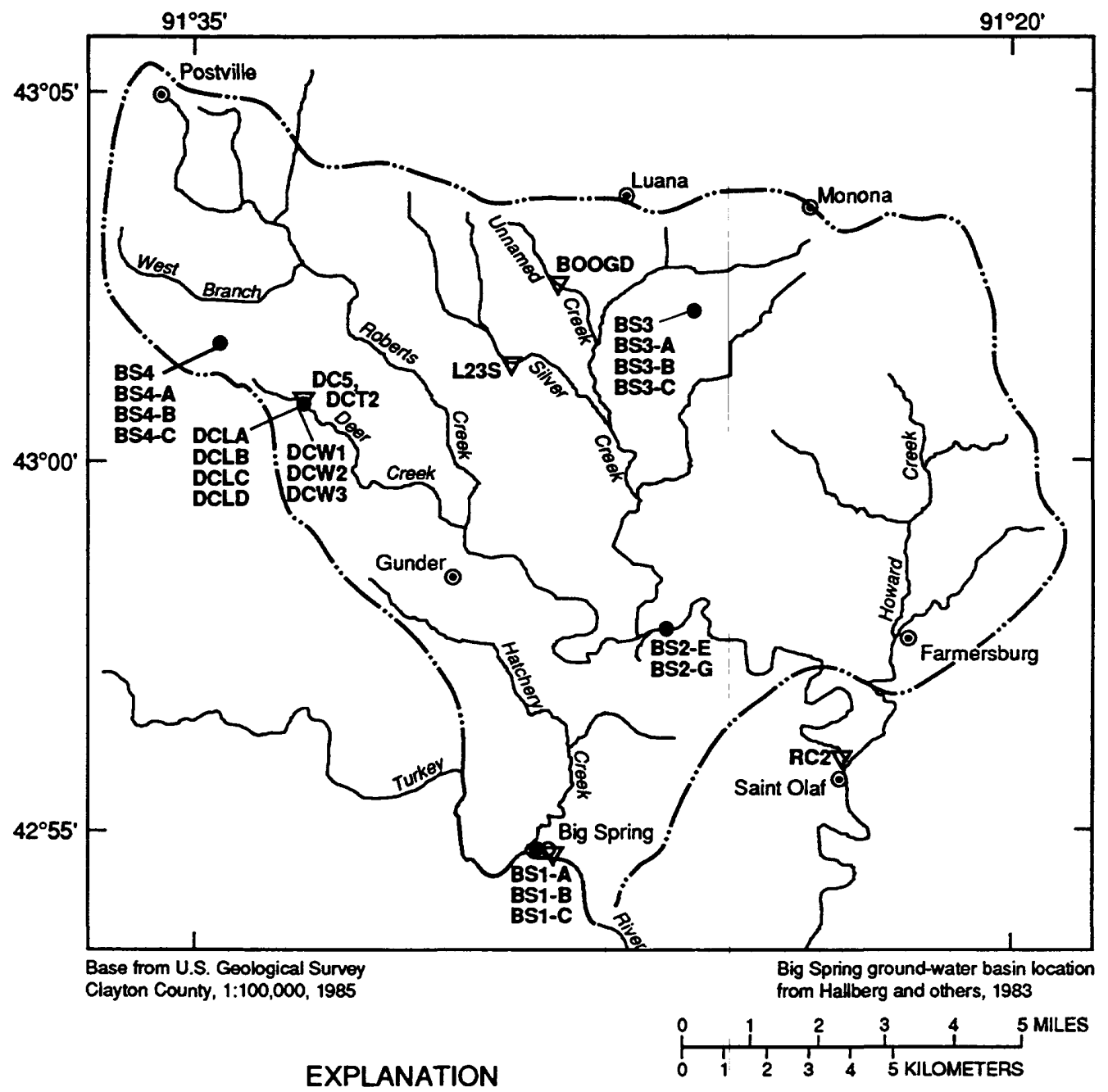

\footnotetext{
—.. BIG SPRING GROUND-WATER BASIN DIVIDE

9 SPRING

${ }^{D C 5} \nabla$ SURFACE-WATER MONITORING SITE AND SITE IDENTIFICATION

DCW1• GROUND-WATER MONITORING SITE AND SITE IDENTIFICATION
}

Figure 2. Location of surface- and ground-water-quality monitoring sites. 
sites are shown in table 1 at the back of the report.

The water-quality constituents continuously monitored in Roberts Creek and Big Spring are specific conductance, $\mathrm{pH}$, and water temperature. These constituents are measured using a multiple-parameter meter and digitally recorded at 15 -minute intervals. The data are retrieved weekly by computer through a telephone modem. Values determined by the multiple-parameter field meter were calibrated weekly against conductance-reference and pH-buffer solutions. Temperature values were calibrated with a mercury thermometer. Stage was recorded continuously at stream sites DC5, RC2, L23S, and BOOGD with bubble-gage sensors and digital recorders (Rantz and others, $1982 a$, p. 32-39). Stage measurements were calibrated by comparison to permanent reference marks. Stream discharge was calculated from stage using stage-discharge relations (Kennedy, 1983, p. 30-32) or by using the theoretical-discharge equation for $V$-notch weirs (Rantz and others, 1982b, p. 305). From the 15-minute observations, daily mean values were calculated and permanently stored in the U.S. Geological Survey National Water Data Storage and Retrieval System (WATSTORE). Stream discharge normally was measured by current-meter methods (Buchanan and Somers, 1969). Because of low-flow conditions with shallow stream depths during most of the 1990 water year, the 0.6 -depth method generally was used.

Water temperatures and dissolved-oxygen concentrations were measured in a flowing section of the stream or spring pool at the time of sample collection. Water temperatures were measured with a standard mercury or alcohol thermometer that had been compared with a laboratory-grade thermometer for accuracy. Dissolved-oxygen concentrations were measured with a dissolved-oxygen meter. Immediately after sample collection, the specific conductance and $\mathrm{pH}$ of the water were measured.

Suspended-sediment samples were collected periodically by local observers, technicians, and by automatic samplers during storms. The observers collected depth- integrated samples at one vertical using techniques described by Guy and Norman (1970). Samples were collected three times per week at Roberts Creek above Saint Olaf (site RC2), intermittently at Unnamed Creek near Luana (site BOOGD), and weekly at Big Spring.

Water levels in the principal water-supply aquifers in the basin were recorded at four well nests (table 2). At each well nest, water levels were recorded for the unconsolidated aquifers, the Galena aquifer, and the Saint Peter aquifer. Water levels were sensed by a float and then recorded hourly for subsequent storage in the WATSTORE data base. Additional wells (DCW1, DCW2, and DCW3) were used to monitor the relation between water levels in the unconsolidated aquifer and flow in Deer Creek.

Water samples for chemical analyses were collected from wells after a minimum of three casing volumes had been pumped from the well or the well had been bailed dry and allowed to recover. At the Deer Creek site, suction lysimeters were installed in the unconsolidated zone. Lysimeters were evacuated by a hand pump and allowed to equilibrate about 24 hours, allowing water to flow into the lysimeter under partial vacuum conditions. The sample was removed by purging with air.

The discharge measurement and waterquality sampling techniques used for the seepage study were the same as used for the routine sampling just described. The location and drainage areas of the seepage study sites are given in table 3. Samples for chemical analyses were prepared for shipment to the laboratory as described in table 4. Analyses of water samples by the University of Iowa Hygienic Laboratories in Iowa City and Des Moines, Iowa, followed the analytical methods listed in table 4. The U.S. Environmental Protection Agency's (1983) method 81.41 for the analyses of pesticides was modified to use dual capillary columns. Water samples collected as part of the degradation study were filtered through a 1-micrometer glass-fiber filter at the time of collection and then were shipped chilled for analysis of herbicide and herbicide metabolite concentrations by the U.S. Geological Survey laboratory in Lawrence, Kans. Samples were analyzed using solid-phase extraction techniques (Thurman and others, 1990). 
Suspended-sediment concentrations were determined by the U.S. Geological Survey laboratory in Iowa City, Iowa, using standard filtration and evaporation methods (Guy,1969).

In this report, a shorthand terminology is used in describing the results of the chemical analyses of nitrogen species and herbicides. The results of the analyses of nitrogen species were reported in concentrations as nitrogen (nitrite plus nitrate as nitrogen, ammonia as nitrogen, and organic nitrogen). To save space and yet show that the results are concentrations as nitrogen, "nitrate- $\mathrm{N}$ " was used for nitrite plus nitrate as nitrogen; "ammonia- $\mathrm{N}$ " is used for ammonia as nitrogen, and "organic- $\mathrm{N}$ " is used for organic nitrogen. A fraction of the herbicides in a sample generally is lost during the extraction procedure. Therefore, results of the analyses are reported in total-recoverable concentrations. No attempt was made to correct herbicide concentrations for recovery rate.

\section{Acknowledgments}

This project was supported, in part, by the Iowa Department of Natural Resources, Geological Survey Bureau, through the Big Spring Basin Demonstration Project, with funds provided from the Iowa Groundwater Protection Act. George Hallberg, Coordinator of the Big Spring Basin Demonstration Project, and other members of the Iowa Department of Natural Resources, Geological Survey Bureau, Robert Libra, John Littke, Debra Quade, and Robert Rowden provided technical advice and field support. Jerry Spykerman, manager of the Big Spring Fish Hatchery, provided support for data collection at the spring and collected precipitation samples. Area residents, Leann Hilgerson, Karen and Eugene Voss, and Jerry Koonze collected sediment samples.

\section{HYDROLOGIC DATA SUMMARY}

\section{Precipitation}

Precipitation was measured and sampled 48 of 52 weeks at Big Spring during water year 1990. Data were not collected from August 21 to September 18 because the rain gage and sampler were damaged by flood waters from the Turkey River. Precipitation data for Roberts Creek above Saint Olaf (site RC2) are complete for the most part, but the precipitation data for Unnamed Creek near Luana (site BOOGD) are incomplete due to malfunctioning equipment in August and September.

\section{Quantity}

Rainfall for the 48 weeks that data were collected at Big Spring totaled 30.36 in., with a median weekly rainfall of 0.26 in. (table 5). An additional $4.19 \mathrm{in}$. were recorded at the nearby National Weather Service station in Elkader, Iowa (National Oceanic and Atmospheric Administration, 1990a, 1990b) during the period the gage was not operational at Big Spring.

Precipitation at Unnamed Creek near Luana (site BOOGD) and at Roberts Creek above Saint Olaf (site RC2) is listed in table 6 . The total rainfall measured for water year 1990 was 43.67 in. at site RC2. The largest monthly rainfall occurred in August when 14.45 in. fell at site RC2. The largest daily rainfall at site RC2 was 5.23 in. on August 25, 1990.

\section{Quality}

Weekly precipitation samples were collected during the 41 weeks when measurable rainfall occurred at Big Spring. Results of chemical analyses of these samples are summarized in table 5. The median concentration of the predominant cation, calcium, was $0.48 \mathrm{mg} / \mathrm{L}$ (milligrams per liter), and the median concentration of the predominant anion, sulfate, was $1.7 \mathrm{mg} / \mathrm{L}$. The median concentrations of nitrate- $\mathrm{N}$ and ammonia- $\mathrm{N}$ were 0.35 and 0.48 $\mathrm{mg} / \mathrm{L}$, respectively. Maximum concentrations of all major ions were less than $10 \mathrm{mg} / \mathrm{L}$.

\section{Surface Water}

\section{Deer Creek}

Discharge and water-quality data were collected at site DC5 on Deer Creek near Postville (fig. 2) to determine the relation between ground and surface water in a small drainage basin.

\section{Discharge}

Stream discharge was continuously recorded from October 1,1989 , to January 4, 
1990 , and from May 3 to the end of the water year (table 7). Daily mean discharge recorded at site DC5 ranged from $0.13 \mathrm{ft}^{3} / \mathrm{s}$ at the end of September 1990 to $2.7 \mathrm{ft}^{3} / \mathrm{s}$ on August 24 after the greatest daily rainfall in the basin. Median monthly discharge ranged from $0.17 \mathrm{ft}^{3} / \mathrm{s}$ in September to $0.30 \mathrm{ft}^{3} / \mathrm{s}$ in June and August 1990.

\section{Water Quality}

Specific conductance of water from Deer Creek ranged from 231 to $680 \mu \mathrm{S} / \mathrm{cm}$ (microsiemens per centimeter at $25^{\circ} \mathrm{C}$ ), $\mathrm{pH}$ from 7.1 to 8.2 units, and dissolved oxygen from 6.7 to $14.5 \mathrm{mg} / \mathrm{L}$ (table 8). Chemical analyses of dissolved constituents in five samples collected from site DC5 on Deer Creek show that calcium and magnesium were the predominant cations in solution (table 9). Calcium concentrations ranged from 68 to $110 \mathrm{mg} / \mathrm{L}$, and magnesium concentrations ranged from 23 to $30 \mathrm{mg} / \mathrm{L}$. Sodium concentrations were less than $6.0 \mathrm{mg} / \mathrm{L}$, and potassium concentrations were $2.1 \mathrm{mg} / \mathrm{L}$ or less. The predominant anion in solution was bicarbonate. Bicarbonate concentrations in three samples ranged from 330 to $380 \mathrm{mg} / \mathrm{L}$. Sulfate concentrations ranged from 3.0 to 34 $\mathrm{mg} / \mathrm{L}$. Chloride concentrations were $12 \mathrm{mg} / \mathrm{L}$ or less. Nitrate- $\mathrm{N}$ was the predominant nitrogen species in 18 of 25 samples, and organic- $N$ was the predominant nitrogen species in 7 samples (table 10). Nitrate-N concentrations ranged from 0.70 to $17 \mathrm{mg} / \mathrm{L}$. Organic-N concentrations ranged from 0.20 to $9.9 \mathrm{mg} / \mathrm{L}$.

Four herbicides, alachlor, atrazine, cyanazine, and metolachlor were detected in 27 samples collected at site DC5 on Deer Creek (fig. 3 and table 11). Alachlor concentrations ranged from less than 0.10 to $0.35 \mu \mathrm{g} / L$ (micrograms per liter) and were greater than $0.10 \mu g / L$ in 7 percent of the samples. Atrazine concentrations ranged from less than 0.10 to $55 \mu \mathrm{g} / L$. Concentrations were equal to or greater than the detection level $(0.10 \mu \mathrm{g} / L)$ in 70 percent of the samples. Cyanazine concentrations ranged from less than 0.10 to $12 \mu \mathrm{g} / L$ and were equal to or greater than $0.10 \mu \mathrm{g} / L$ in about 26 percent of the samples. Metolachlor concentrations ranged from less than 0.10 to $69 \mu \mathrm{g} / L$ and were equal to or greater than $0.10 \mu \mathrm{g} / L$ in about 30 percent of the samples. Metribuzin, butylate, and trifluralin were not detected.
Several samples also were collected for the analysis of the stable isotopes hydrogen 2 (deuterium) and oxygen 18. The results of the analyses are shown graphically in figure 4 and are listed in table 12.

\section{Silver Creek}

Flow in Silver Creek at site L23S ceased from December 12, 1989, through January 7, 1990 , and for 16 days during the period from January 12 to February 4, 1990 (fig. 5 and table 13). The maximum daily mean discharge (107 $\mathrm{ft}^{3} / \mathrm{s}$ ) was recorded on August 25, 1990. Daily mean discharge exceeded $1.0 \mathrm{ft}^{3} / \mathrm{s} 23$ percent of the year and exceeded $0.10 \mathrm{ft}^{3} / \mathrm{s}$ approximately 70 percent of the year (fig. 6).

\section{Unnamed Creek}

\section{Discharge}

Generally there was no streamflow in Unnamed Creek near Luana (site BOOGD) from October 1, 1989, to February 4, 1990, and from March 16 to June 13, 1990 (table 14). The maximum daily mean discharge at site BOOGD was $21 \mathrm{ft}^{3} / \mathrm{s}$ on August 25, 1990. Daily mean discharge exceeded $1.0 \mathrm{ft}^{3} / \mathrm{s} 5$ percent of the year and exceeded $0.10 \mathrm{ft}^{3} / \mathrm{s}$ approximately 22 percent of the year (fig. 6). There was no streamflow 56 percent of the days during water year 1990.

\section{Water Quality}

Collection of water-quality data was limited at Unnamed Creek near Luana (site BOOGD) due to lack of streamflow. Monthly samples were collected in February, March, July, August, and September 1990, and additional samples were collected during snowmelt and five rains. Nitrate- $\mathrm{N}$ concentrations ranged from 1.0 to $30 \mathrm{mg} / \mathrm{L}$ (table 10). Ammonia-N concentrations ranged from less than 0.10 to 5.3 $\mathrm{mg} / \mathrm{L}$. Organic- $\mathrm{N}$ concentrations ranged from 0.8 to $14 \mathrm{mg} / \mathrm{L}$.

Four herbicides were detected in 14 samples from site BOOGD (table 11). Alachlor was detected in about 36 percent of the samples; atrazine in all samples; cyanazine in 64 percent of the samples; and metolachlor in 36 percent of the samples. Alachlor concentrations ranged from less than 0.10 to $8.6 \mu \mathrm{g} / L$; atrazine from 

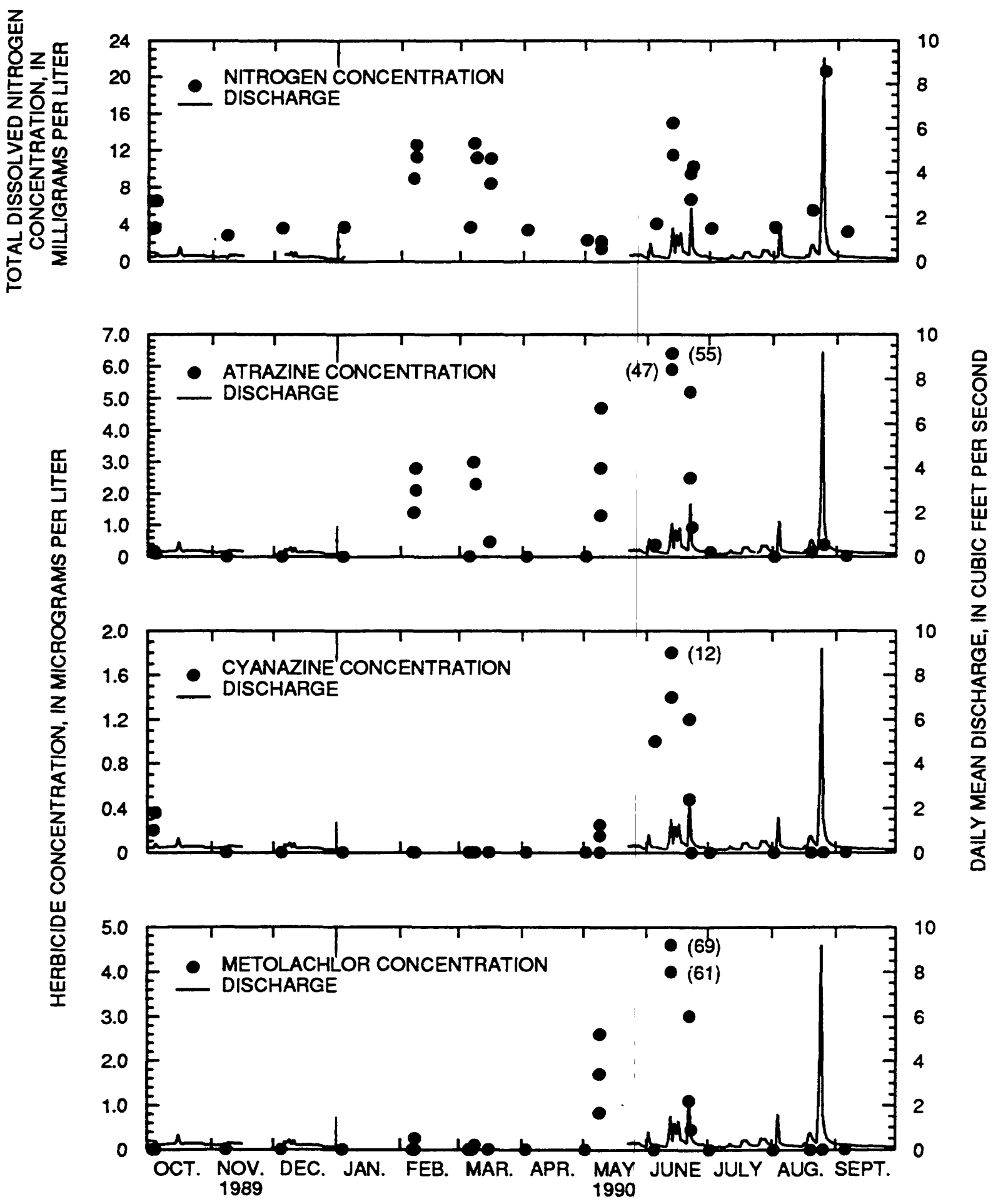

\section{EXPLANATION}

(55) HERBICIDE CONCENTRATIONIn micrograms per liter

Figure 3. Selected chemical constituents and stream discharge at site DC5, Deer Creek near Postville, lowa, water year 1990. 


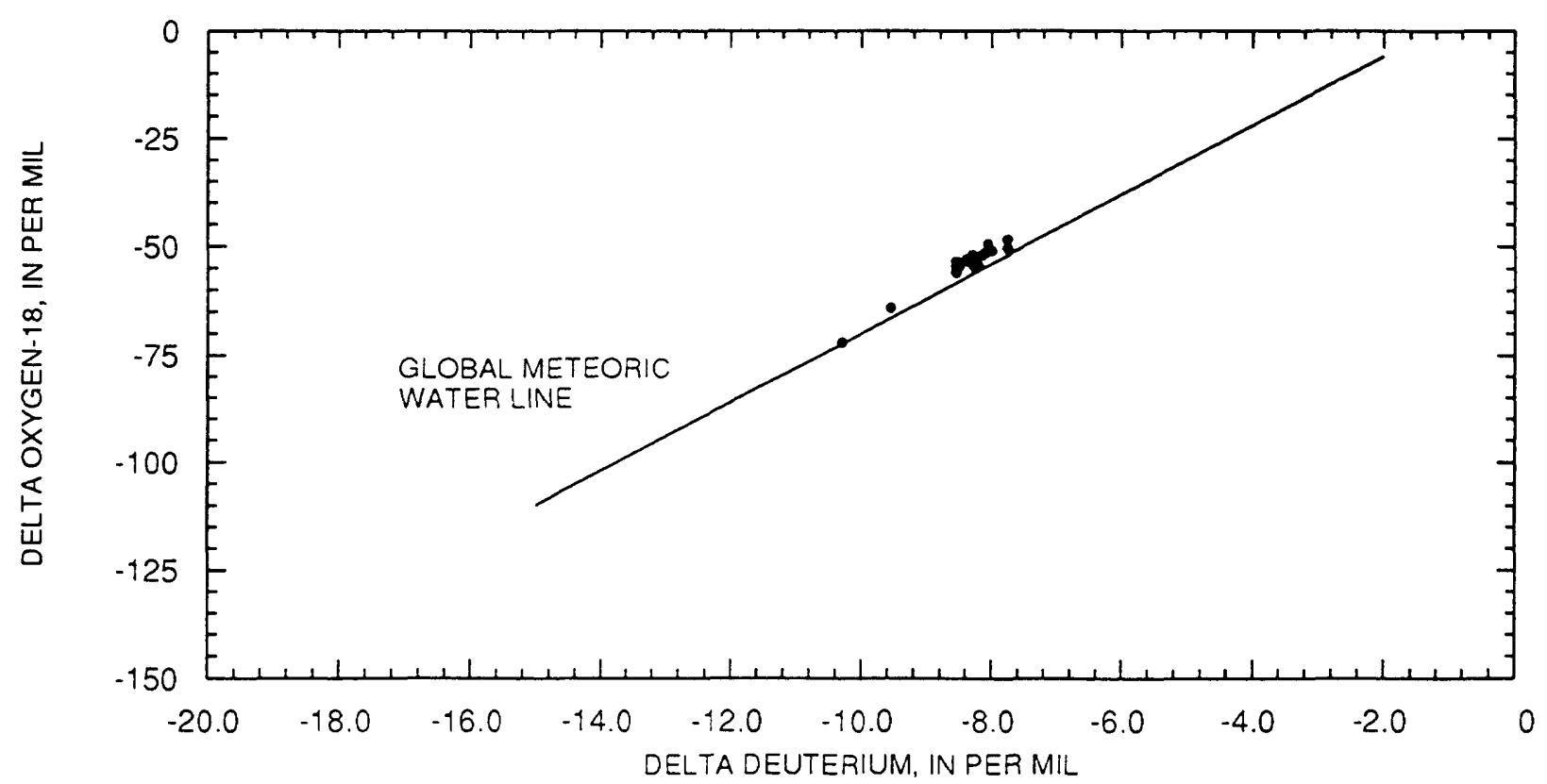

Figure 4. Relation of deuterium to oxygen-stable isotopes at sites in the Deer Creek watershed, water year 1990.

0.46 to $19 \mu \mathrm{g} / L$; cyanazine from less than 0.10 to $7.2 \mu \mathrm{g} / \mathrm{L}$; and metolachlor from less than 0.10 to $3.8 \mu \mathrm{g} / L$.

The daily mean suspended-sediment concentrations ranged from $9 \mathrm{mg} / \mathrm{L}$ on September 14 to $1,140 \mathrm{mg} / \mathrm{L}$ on June 13 and 15 , 1990. The daily suspended-sediment load ranged from 0 on days of no streamflow to 8.7 ton/d on March 11 during snowmelt (fig. 7 and table 15).

\section{Roberts Creek}

\section{Discharge}

Daily mean discharge at Roberts Creek above Saint Olaf (site RC2) is listed in table 16 and illustrated in figure 8 . The median discharge at site RC2 for the 1990 water year was $2.4 \mathrm{ft}^{3} / \mathrm{s}$. Maximum daily mean discharge (426 $\left.\mathrm{ft}^{3} / \mathrm{s}\right)$ occurred on August 25, 1990. Generally there was no recorded discharge from November 2, 1989, through January 9, 1990. No streamflow was recorded 22 percent of the days in water year 1990. Flow duration is shown in figure 6. Mean daily discharge exceeded 0.10 $\mathrm{ft}^{3} / \mathrm{s}$ approximately 71 percent of the time during water year 1990 , exceeded $1.0 \mathrm{ft}^{3} / \mathrm{s}$ approximately 60 percent of the time, and exceeded $10 \mathrm{ft}^{3} / \mathrm{s}$ approximately 16 percent of the time.
Water Quality

The maximum daily mean specific conductance of $797 \mu \mathrm{S} / \mathrm{cm}$ was recorded in Roberts Creek above Saint Olaf (site RC2) on August 29 (table 16). The minimum daily mean specific conductance $(512 \mu \mathrm{S} / \mathrm{cm})$ was recorded on July 20 . The daily mean water temperature varied from $2.0^{\circ} \mathrm{C}$ on March 23,1990 , to $27.5^{\circ} \mathrm{C}$ on July 4. Daily median $\mathrm{pH}$ varied from 6.6 on October 5, 1989, to 9.1 on April 13 and 17 (table 17).

Six samples were collected from site RC2 on Roberts Creek during water year 1990 for analyses of major ions. Calcium and magnesium were the predominant cations. Bicarbonate was the predominant anion in solution (table 9). Sodium and potassium concentrations generally were less than $20 \mathrm{mg} / \mathrm{L}$. Sulfate concentrations ranged from 23 to $46 \mathrm{mg} / \mathrm{L}$, and chloride concentrations ranged from 25 to $35 \mathrm{mg} / \mathrm{L}$. Silica concentrations were $12 \mathrm{mg} / \mathrm{L}$ or less.

Forty-two samples were collected from site RC2 on Roberts Creek for the analyses of nitrogen species, orthophosphorus, and organic-carbon concentrations (table 10). Total nitrate- $\mathrm{N}$ concentrations were greater than the detection level $(0.10 \mathrm{mg} / \mathrm{L})$ in all but one of the samples. Nitrate- $\mathrm{N}$ concentrations ranged from less than 0.10 to $18 \mathrm{mg} / \mathrm{L}$. Ammonia-N was detected in 68 percent of the samples. Total 


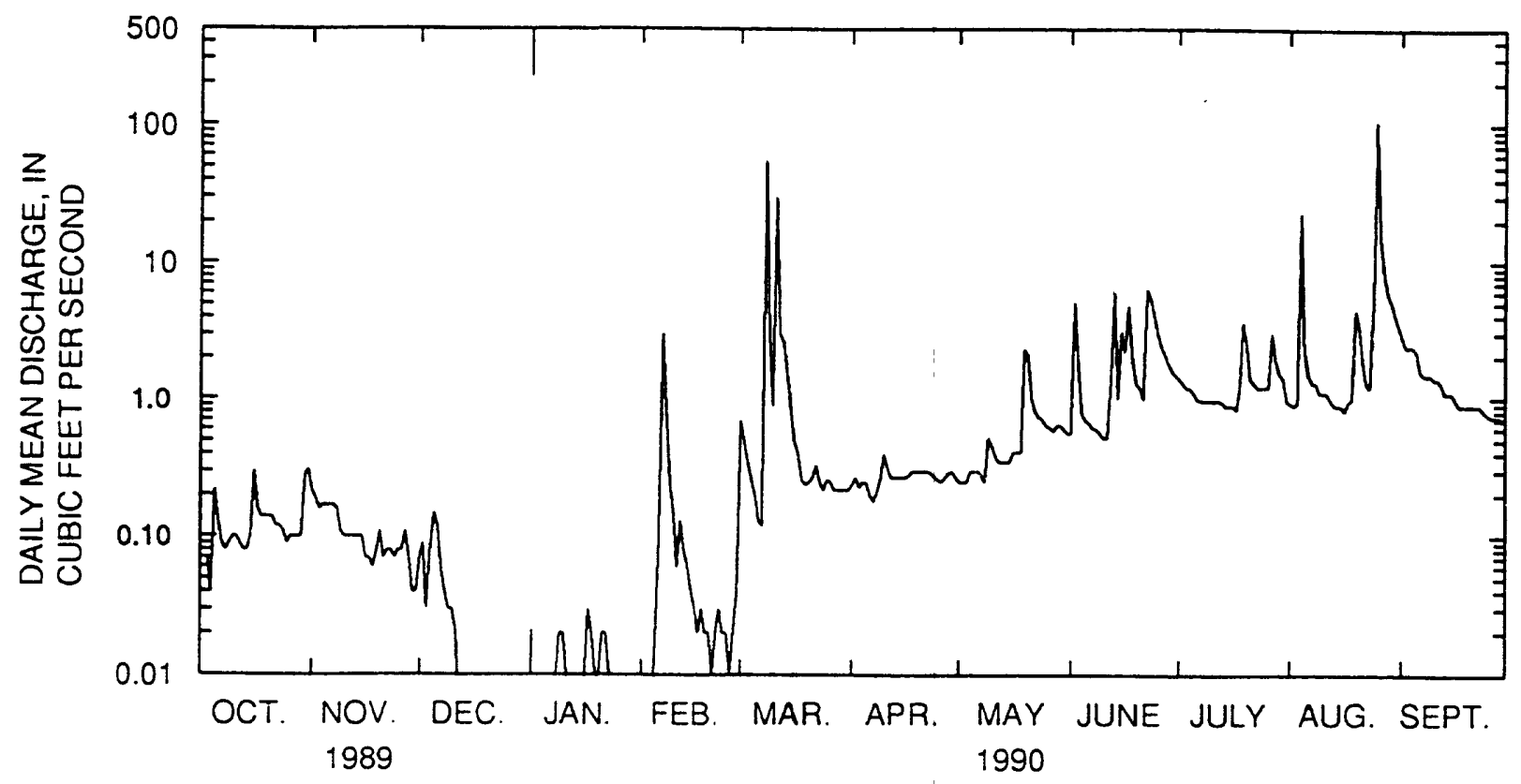

Figure 5. Daily mean discharge at site L23S, Silver Creek near Luana, lowa, water year 1990.

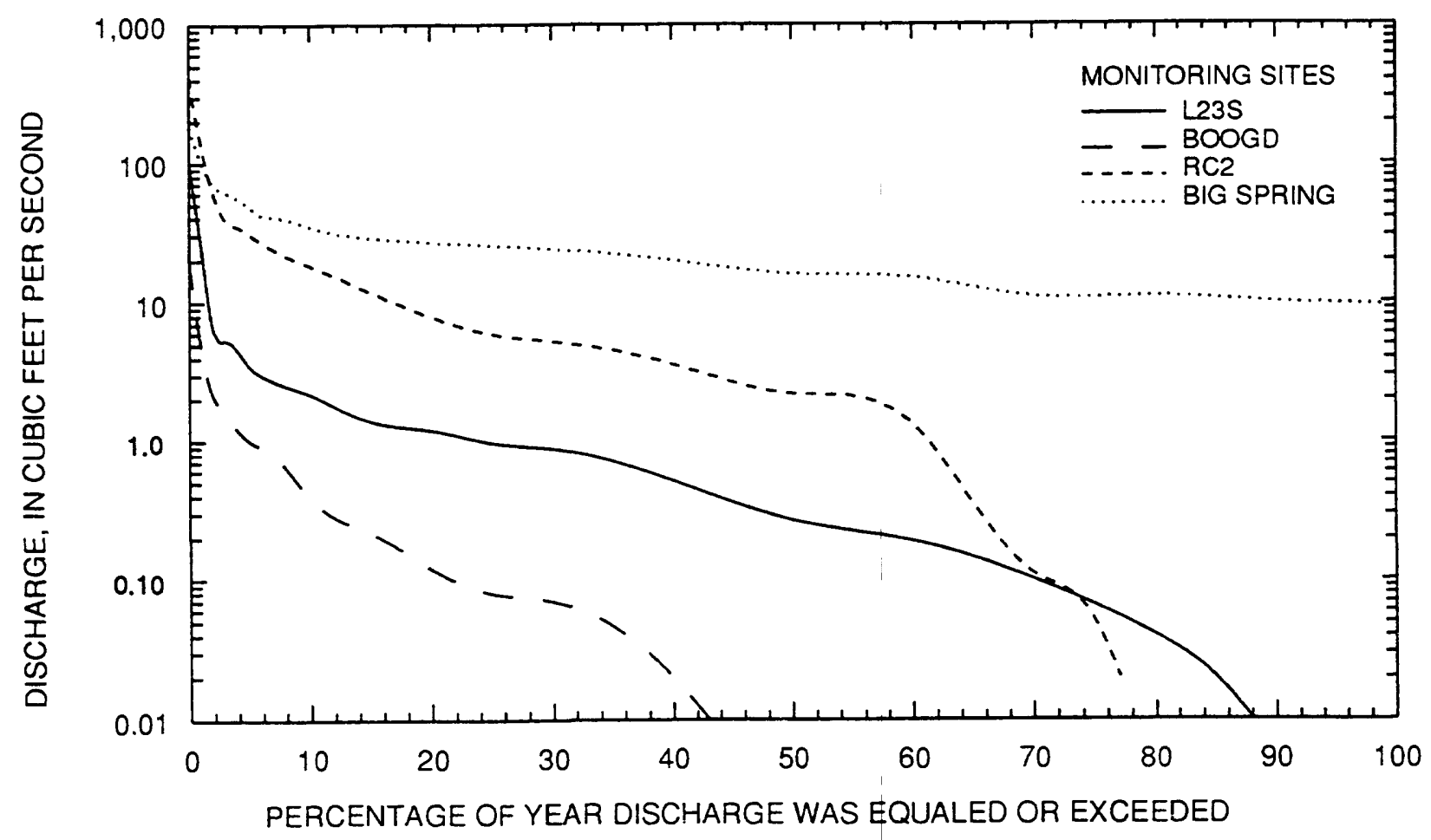

Figure 6. Flow duration at monitoring sites in the Big Spring basin, water year 1990. 


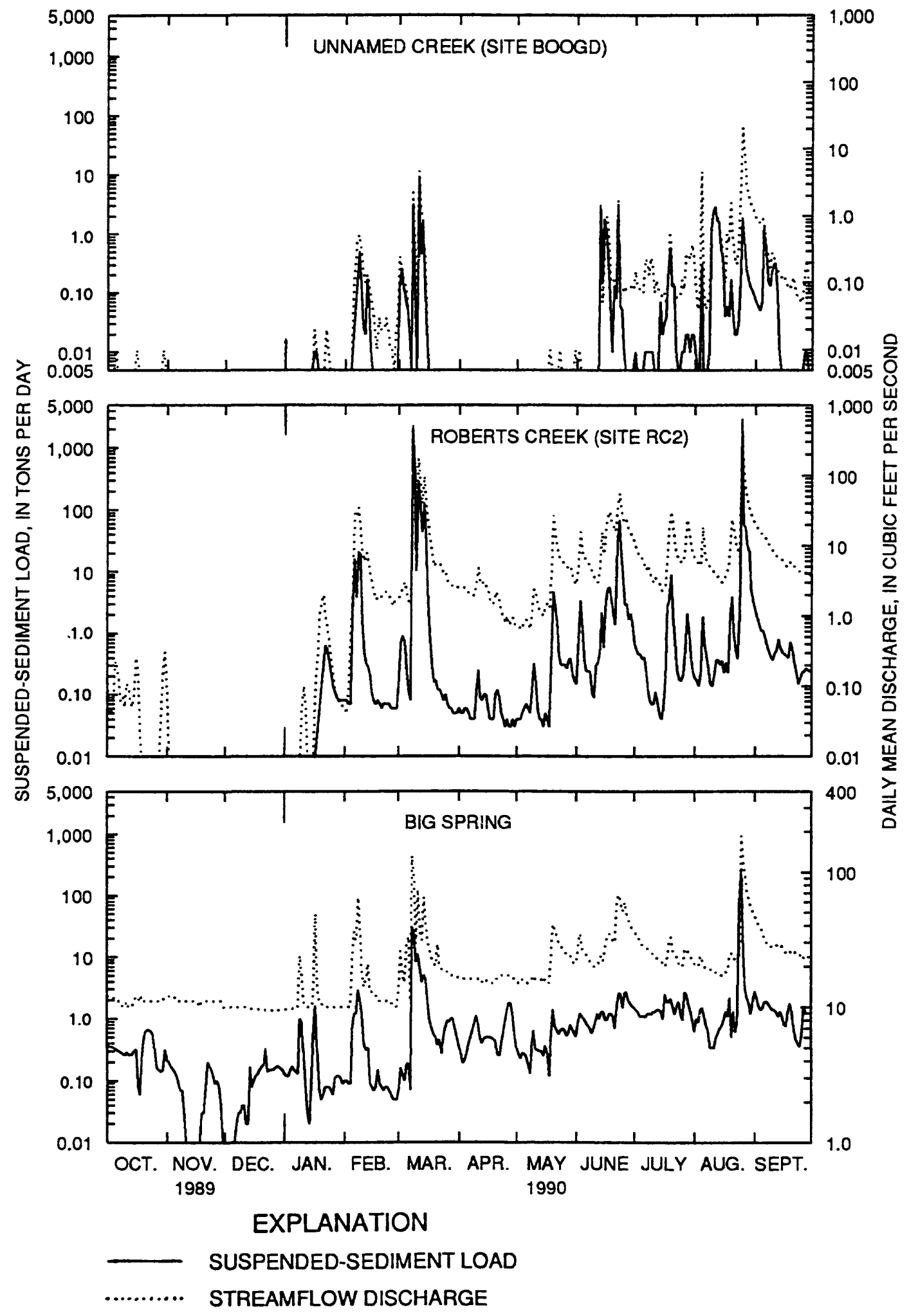

Figure 7. Suspended-sediment load and streamflow discharge at three monitoring sites in the Big Spring basin, water year 1990. 

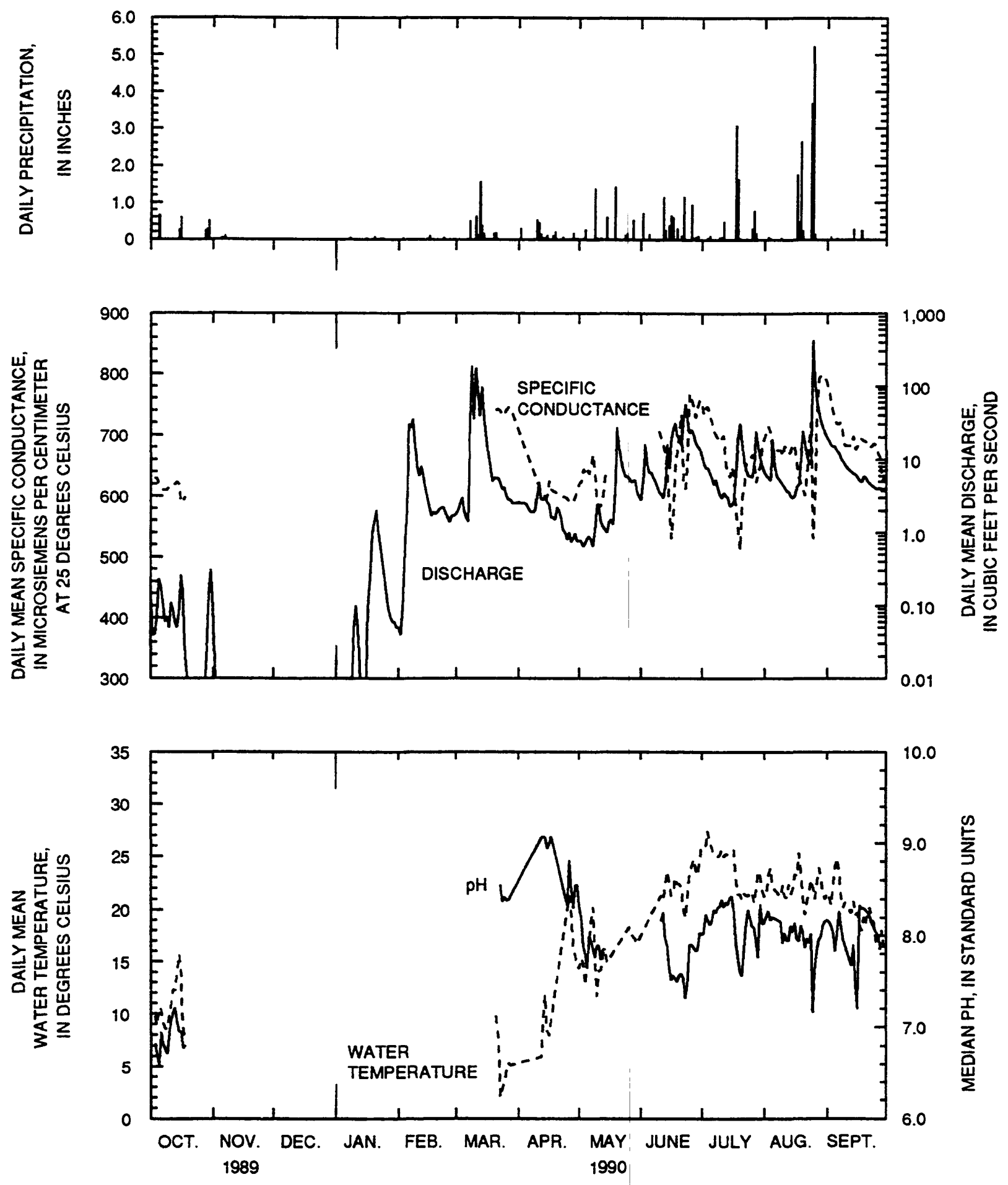

Figure 8. Daily precipitation, daily mean specific conductance, discharge, water temperature, and daily median pH at site RC2, Roberts Creek above Saint Olaf, lowa, water year 1990. 
ammonia- $N$ concentrations ranged from less than 0.10 to $4.0 \mathrm{mg} / \mathrm{L}$. Total organic-N concentrations ranged from 0.50 to $20 \mathrm{mg} / \mathrm{L}$. Total orthophosphorus concentrations ranged from 0.20 to $2.1 \mathrm{mg} / \mathrm{L}$.

Thirty-four samples were collected from site RC2 on Roberts Creek for the analyses of seven herbicides. Five of the seven herbicides were detected (concentration greater than 0.10 $\mu g / L)$. Alachlor was detected in 56 percent of the samples; atrazine in 100 percent; cyanazine in about 68 percent; metolachlor in 47 percent; and metribuzin in 6 percent of the samples (table 11). Butylate and trifluralin were not detected. Alachlor concentrations ranged from less than 0.10 to $8.2 \mu \mathrm{g} / L$; atrazine from 0.16 to $12 \mu \mathrm{g} / L$; cyanazine from less than 0.10 to 8.5 $\mu g / L$, and metolachlor from less than 0.10 to 7.4 $\mu g / L$.

The daily mean suspended-sediment loads at site RC2 ranged from 0 on days of no streamflow to more than 2,900 ton/d on August 25 (fig. 7 and table 18). The largest monthly sediment loads were in March (more than 3,400 tons) and August (more than 3,100 tons).

\section{Ground Water}

Water levels were recorded continuously at five wells screened in unconsolidated material in the Big Spring basin. Well BS1-A is screened in alluvial material in the Turkey River valley; well BS3-C in weathered limestone and loess; wells BS4-B and BS4-C in glacial till of Pre-Illinoian age; and well DCW1 in Deer Creek alluvial material. Water levels in the Galena aquifer were recorded continuously at four monitoring wells (BS1-B, BS2-E, BS3-A, and BS4-A), and water-quality data were collected at the discharge point, Big Spring. Water levels in the Saint Peter aquifer were monitored continuously at two wells (BS2-G and BS4), and two wells (BS1-D and BS3) were measured monthly (table 19).

\section{Unconsolidated Aquifers}

The highest water levels in the unconsolidated aquifers were recorded during August or September 1990 (table 20). The highest recorded water levels in water year 1990 were $2.45 \mathrm{ft}$ below land surface in well BS1-A, $9.88 \mathrm{ft}$ below land surface in well BS3-C, $72.64 \mathrm{ft}$ below land surface in well BS4-B, $55.78 \mathrm{ft}$ below land surface in well BS4-C, and $3.09 \mathrm{ft}$ below land surface in well DCW1. Water levels in well BS1-A (fig. 9) were the highest in August (2.45 $\mathrm{ft}$ below land surface) and the lowest in December 1989 (14.74 ft below land surface). Data were lost from well BS1-A from August 27 through September 5 because the recorder was inundated when the Turkey River flooded. The lowest water level in well BS3-C was $14.84 \mathrm{ft}$ below land surface on October 19, and the lowest water level in well BS4-B was $73.78 \mathrm{ft}$ below land surface on March 6 and 7, 1990. The lowest water level in well BS4-C was $57.06 \mathrm{ft}$ below land surface on February 24, and the lowest water level in well DCW1 was $6.31 \mathrm{ft}$ below land surface on October 3, 1989.

As part of an investigation to study the relation between shallow ground water and surface water, samples were collected for chemical analyses from two wells (DCW2 and DCW3) and four suction lysimeters (DCLA, DCLB, DCLC, and DCLD) completed in unconsolidated material in the Deer Creek watershed. The discharge from a tile line that drains the unconsolidated material in the Deer Creek watershed was also sampled (site DCT2). The location and well-construction data for the wells and lysimeters are given in table 2 . Onsite determinations of selected water-quality constituents in water from wells DCW2 and DCW3 and the tile line are listed in table 21.

Five samples were collected from each well (DCW2 and DCW3) for the analysis of major ions. In samples from both wells, calcium and magnesium were the predominant cations in solution (table 22). Calcium concentrations ranged from 3.4 to $140 \mathrm{mg} / \mathrm{L}$, and magnesium concentrations ranged from 1.6 to $38 \mathrm{mg} / \mathrm{L}$. Sodium and potassium concentrations were less than $10 \mathrm{mg} / \mathrm{L}$. Bicarbonate was the predominant anion. Bicarbonate concentrations ranged from 360 to $460 \mathrm{mg} / \mathrm{L}$. Sulfate concentrations ranged from 8.8 to $96 \mathrm{mg} / \mathrm{L}$, and chloride concentrations ranged from 1.0 to $38 \mathrm{mg} / \mathrm{L}$.

Thirteen samples were collected from each of wells DCW2 and DCW3 for the analysis of nitrogen species, orthophosphorus, and organic carbon and for the analyses of seven herbicides (table 23). Nitrate-N was detected in 62 percent of the samples from well DCW2 and from 31 


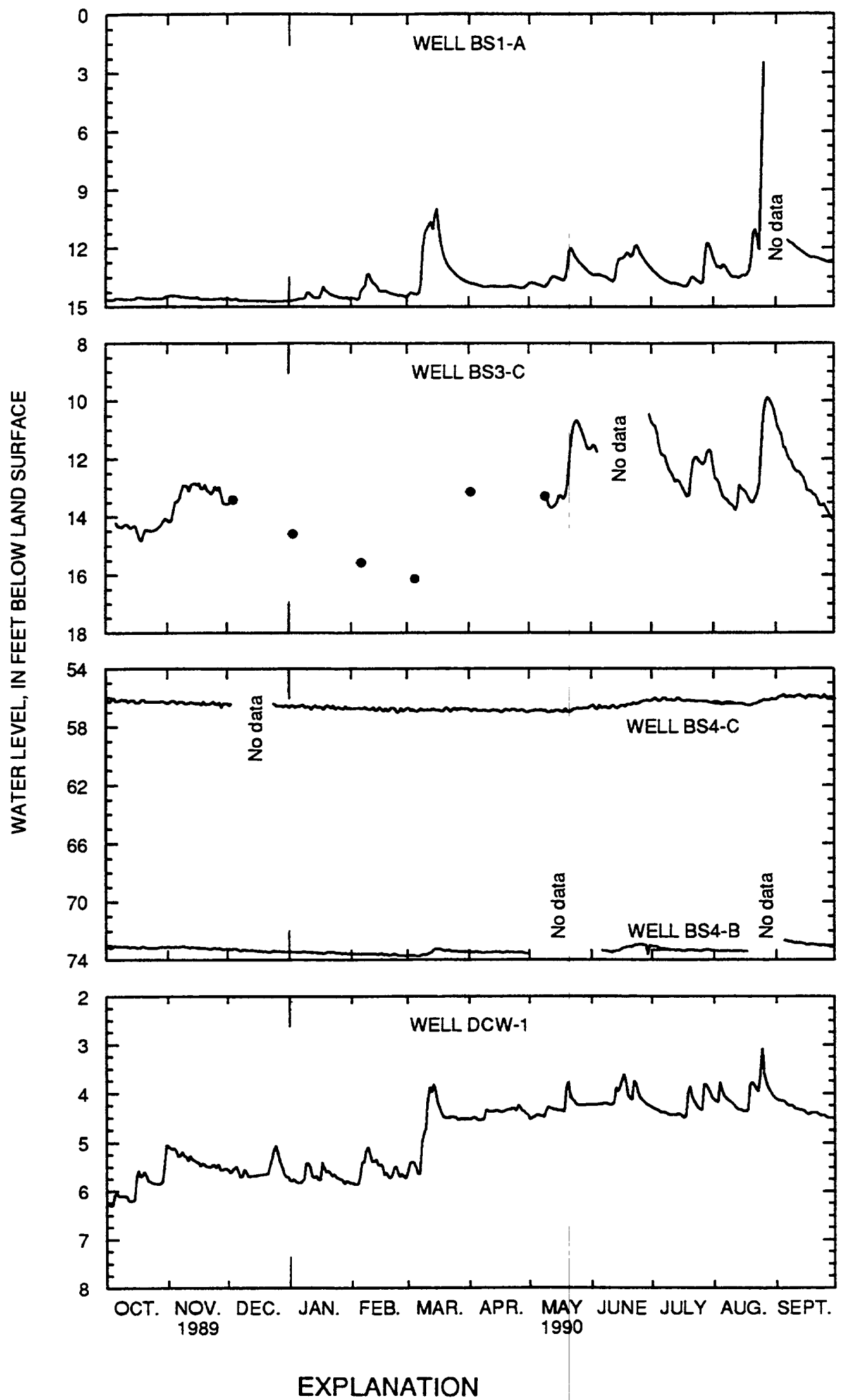

- ISOLATED WATER-LEVEL MEASUREMENT

Figure 9. Daily mean water levels in unconsolidated aquifers in the Big Spring basin, water year 1990. 
percent of the samples from well DCW3. Nitrate- $\mathrm{N}$ concentrations in well DCW2 ranged from less than 0.10 to $16 \mathrm{mg} / \mathrm{L}$ and ranged from less than 0.10 to $0.10 \mathrm{mg} / \mathrm{L}$ in well $\mathrm{DCW} 3$. Ammonia- $N$ concentrations were greater than the detection level $(0.10 \mathrm{mg} / \mathrm{L})$ in 15 percent of samples from well DCW2 and were greater than the detection level in 77 percent of the samples from well DCW3. The largest concentration was $0.80 \mathrm{mg} / \mathrm{L}$ in a sample from well DCW2. Organic- $\mathrm{N}$ concentrations ranged from less than 0.10 to $0.60 \mathrm{mg} / \mathrm{L}$. Orthophosphorus concentrations were less than the detection level $(0.10 \mathrm{mg} / \mathrm{L})$ in 81 percent of the samples. Atrazine, cyanazine, and metolachlor were detected in wells DCW2 and DCW3 (table 24). Atrazine was detected in 46 percent of the samples from well DCW2 and in 15 percent of the samples from well DCW3. Atrazine concentrations ranged from less than 0.10 to 2.4 $\mu g / L$ in well DCW2 and in less than 0.10 to 0.75 $\mu g / L$ in well DCW3. Cyanazine was detected in 15 percent of the samples from well DCW2 and in one sample from well DCW3.

Four lysimeters were used to collect water from the water table or unsaturated zone depending on the water level at the time of sampling. Lysimeters DCLA and DCLB were always in the saturated zone, lysimeter DCLC was in the saturated zone after October 16, 1989 , and DCLD was in the saturated zone from March 10, 1990, through the remainder of the water year.

Nitrate- $N$ concentrations in the deepest lysimeters, DCLA and DCLB, were less than the detection level in all but three samples (table 23). Nitrate- $N$ concentrations in one sample from DCLA and two samples from DCLB were equal to or greater than the detection level. The maximum nitrate- $\mathrm{N}$ concentration in these two lysimeters was $0.70 \mathrm{mg} / \mathrm{L}$. Nitrate-N concentrations in the two shallowest lysimeters, DCLC and DCLD, were greater than the detection level in all but one sample and ranged from less than 0.10 to $36 \mathrm{mg} / \mathrm{L}$. Nitrate-N concentrations in samples from lysimeter DCLC ranged from less than 0.10 to $0.50 \mathrm{mg} / \mathrm{L}$, and concentrations in samples from lysimeter DCLD ranged from 1.8 to $36 \mathrm{mg} / \mathrm{L}$.

Ammonia- $N$ concentrations were less than the detection level $(0.10 \mathrm{mg} / \mathrm{L})$ in all but one sample from all lysimeters. Organic-N concentrations in the four lysimeters ranged from less than 0.10 to $0.90 \mathrm{mg} / \mathrm{L}$. Total nitrogen concentrations (nitrate-N, ammonia- $\mathrm{N}$, and organic-N) in samples from the lysimeters and the water level in well DCW1 are shown in figure 10.

Atrazine was the only herbicide detected in the lysimeter (DCLA) installed $8.5 \mathrm{ft}$ below land surface (table 24). In lysimeter DCLB, which was installed at $7 \mathrm{ft}$ below land surface, only alachlor and cyanazine were detected. Atrazine was the only herbicide detected in samples from lysimeter DCLC, the lysimeter installed at $5.5 \mathrm{ft}$ below land surface. Atrazine concentrations ranged from less than 0.10 to $0.59 \mu \mathrm{g} / L$. Both atrazine and metolachlor were detected in samples from lysimeter DCLD. All samples had detectable atrazine concentrations, and five of nine samples had detectable metolachlor concentrations. Atrazine concentrations ranged from 0.12 to $3.6 \mu \mathrm{g} / L$, and metolachlor concentrations ranged from less than 0.10 to $0.83 \mu \mathrm{g} / L$ in samples from lysimeter DCLD. Atrazine and metolachlor concentrations in lysimeter water samples and the water level in well DCW1 are shown in figures 11 and 12.

Eleven samples were collected from tile-line discharge (site DCT2) in the Deer Creek watershed for the analyses of nitrogen species and for the analyses of seven selected herbicides. Nitrate- $\mathrm{N}$ concentrations ranged from 4.1 to $42 \mathrm{mg} / \mathrm{L}$, and organic-N concentrations ranged from 0.60 to $2.1 \mathrm{mg} / \mathrm{L}$. Ammonia- $\mathrm{N}$ was not detected (concentrations greater than $0.10 \mathrm{mg} / \mathrm{L}$ ). Two of the seven herbicides were detected. Atrazine concentrations were greater than the detection level in 8 of the 11 samples. Atrazine concentrations ranged from less than 0.10 to $0.48 \mu g / L$. Metolachlor concentrations were greater than the detection level $(0.10 \mu g / L)$ in 2 of 11 samples, and the maximum concentration was $0.85 \mu \mathrm{g} / L$.

Several samples also were collected from wells DCW2 and DCW3 and from the tile line (site DCT2), for the analyses of the stable isotopes deuterium and oxygen 18 . The results of the analyses are shown graphically in figure 4 and are listed in table 12. 

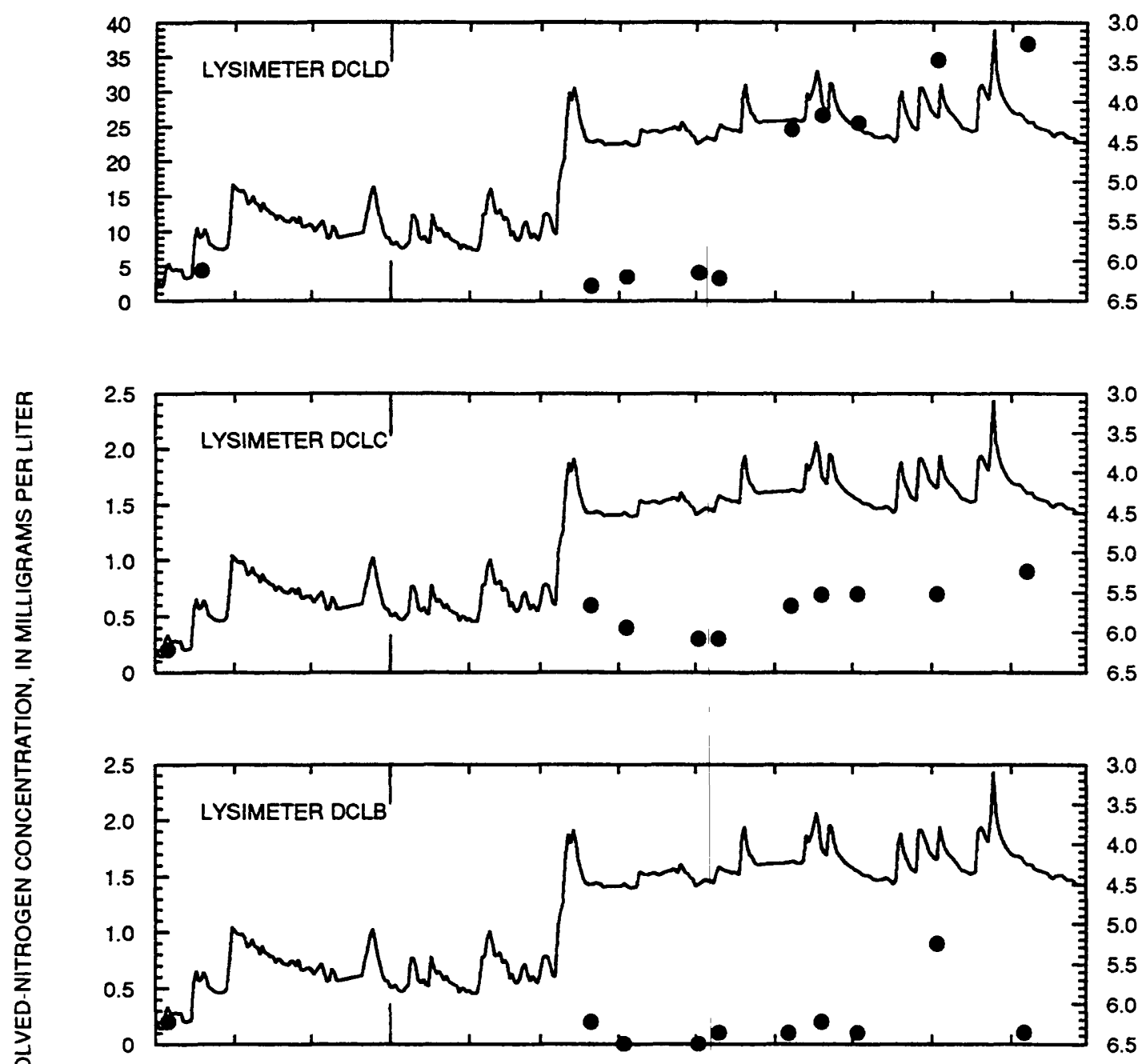

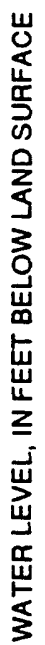

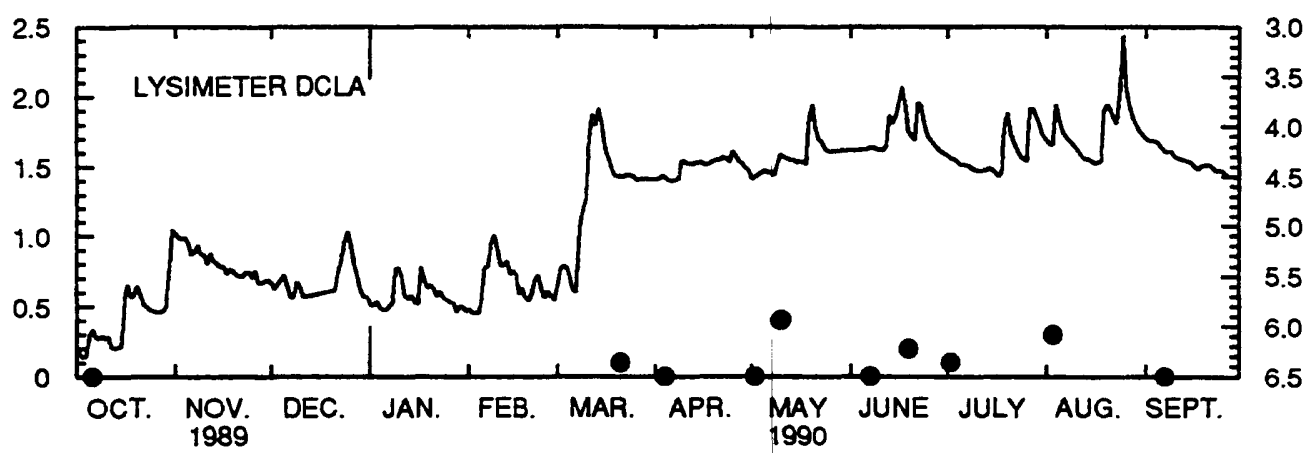

EXPLANATION

WATER LEVEL IN WELL DCW1
TOTAL DISSOLVED-NITROGEN
CONCENTRATION

Figure 10. Total dissolved-nitrogen concentrations in water from lysimeters and water levels in well DCW1 in the unconsolidated aquifer of the Deer Creek watershed, water year 1990. 

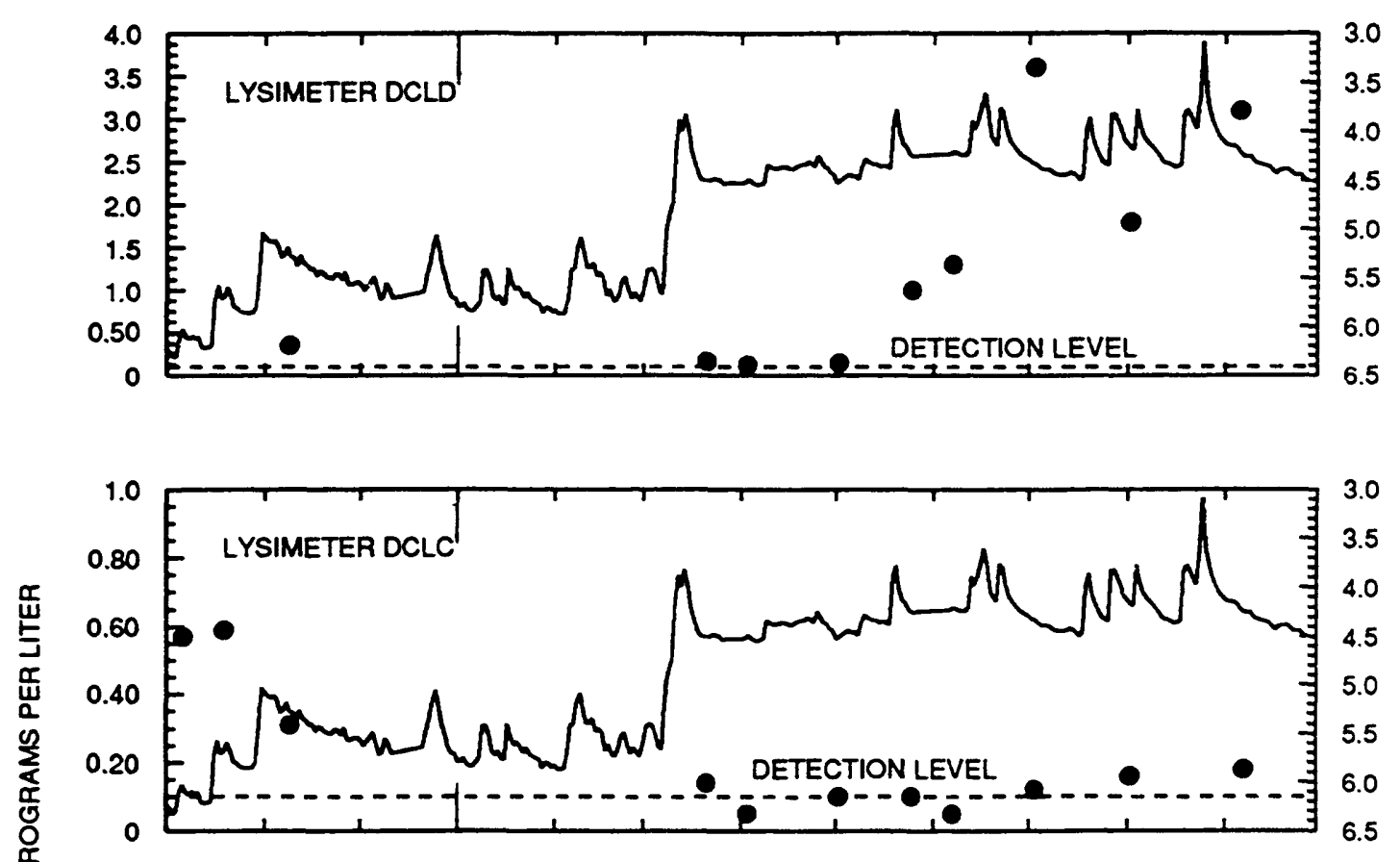

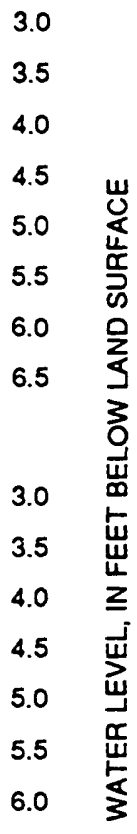

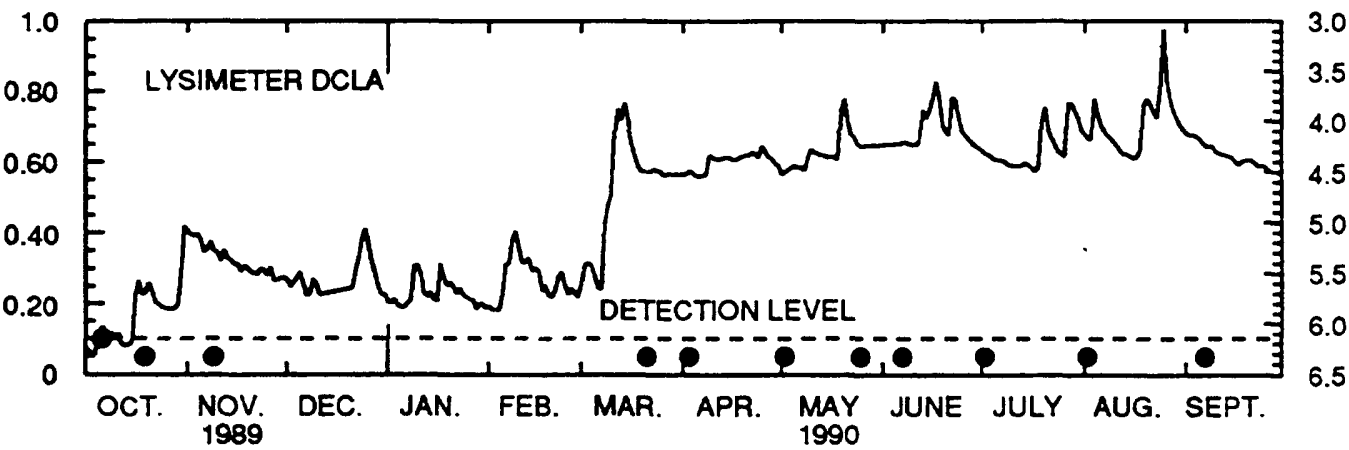

EXPLANATION

WATER LEVEL N NELL DCW 1
TOTAL RECOVERABLE ATRAZINE
CONCENTRATION

Figure 11. Total-recoverable atrazine concentrations in water from lysimeters and water levels in well DCW1 in the unconsolidated aquifer of the Deer Creek watershed, water year 1990. 

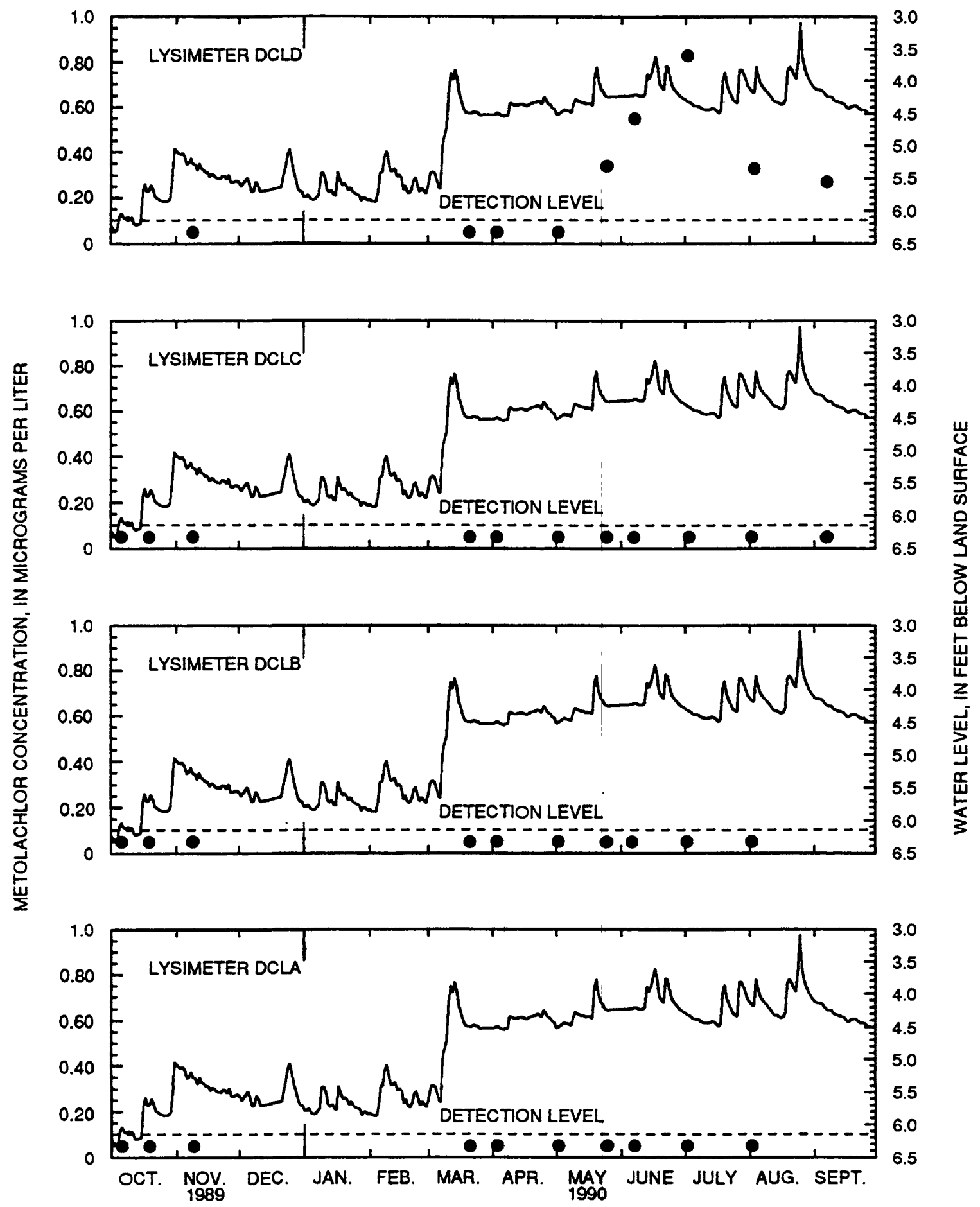

\section{EXPLANATION}

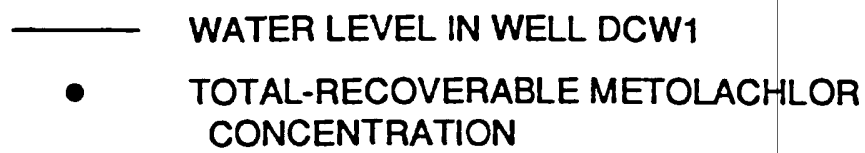

Figure 12. Total-recoverable metolachlor concentrations in water from lysimeters and water levels in well DCW1 in the unconsolidated aquifer of the Deer Creek watershed, water year 1990. 


\section{Galena Aquifer}

Water Levels

The highest water levels in the Galena aquifer were measured in August or September 1990 except for the water levels in well BS1-B (table 25 and fig. 13). The highest daily mean water level in well BS1-B was $3.86 \mathrm{ft}$ below land surface on March 16. The water-level data for this well were lost from August 25 through September 17 because the recorder was damaged by flooding of the Turkey River. The highest daily mean water levels in well BS2-E were $142.54 \mathrm{ft}$ below land surface; $163.58 \mathrm{ft}$ below land surface in well BS3-A, and $215.75 \mathrm{ft}$ below land surface in well BS4-A. The lowest water levels in well BS1-B were $10.34 \mathrm{ft}$ below land surface on January 4, 1990; in well BS2-E, $154.84 \mathrm{ft}$ below land surface on January 4, 1990; in well BS3-A, $168.17 \mathrm{ft}$ below land surface on November 28 and December 7, 1989; and in well BS4-A, $221.88 \mathrm{ft}$ below land surface on February 25, 1990.

\section{Big Spring}

Discharge from Big Spring was continuously recorded and is shown in figure 14 and listed in table 26. The discharge values are the sum of discharge from the main spring and from an auxiliary spring (Back Spring) located about 500 $\mathrm{ft}$ east of the main spring. The maximum daily mean discharge was $187 \mathrm{ft}^{3} / \mathrm{s}$ on August 25, 1990 , and the minimum daily mean discharge was $9.4 \mathrm{ft}^{3} / \mathrm{s}$ on December 26, 1989. Mean daily discharge equaled or exceeded $10 \mathrm{ft}^{3} / \mathrm{s}$ approximately 92 percent of the year, equaled or exceeded $20 \mathrm{ft}^{3} / \mathrm{s}$ approximately 40 percent of the year, and equaled or exceeded $30 \mathrm{ft}^{3} / \mathrm{s}$ approximately 14 percent of the year. The flow duration is shown in figure 6 .

Continuous water-quality data collected at Big Spring are shown as daily mean and median values in figure 14 and are listed in tables 26 and 27. Daily mean specific-conductance values ranged from $378 \mu \mathrm{S} / \mathrm{cm}$ on March 9 during snowmelt to $796 \mu \mathrm{S} / \mathrm{cm}$ on February 6,1990 . The maximum daily median $\mathrm{pH}$ was 7.2 on December 24 through December 26, 1989, and the minimum daily median $\mathrm{pH}$ was 6.5 on March 13, 1990. Daily mean water temperatures ranged from $5.5^{\circ} \mathrm{C}$ on March 9 during snowmelt to $11.5^{\circ} \mathrm{C}$ on August 26 and
27, 1990. Daily mean suspended-sediment concentrations ranged from 0 on 8 days from November 11 through December 5, 1989, to $5,100 \mathrm{mg} / \mathrm{L}$ on August 25, 1990 (table 28). The daily suspended-sediment load ranged from 0 to 275 ton/d. The greatest monthly sediment loads were about 400 tons in August and about 100 tons in March 1990.

The predominant cations in six samples collected at Big Spring generally were calcium and magnesium (table 22). Calcium concentrations ranged from 40 to $91 \mathrm{mg} / \mathrm{L}$, and magnesium concentrations ranged from 16 to 40 $\mathrm{mg} / \mathrm{L}$. Sodium and potassium concentrations were $25 \mathrm{mg} / \mathrm{L}$ or less. The predominant anion, bicarbonate, was present in concentrations that ranged from 330 to $400 \mathrm{mg} / \mathrm{L}$. Sulfate concentrations ranged from 29 to $43 \mathrm{mg} / \mathrm{L}$, and chloride concentrations ranged from 15 to 32 $\mathrm{mg} / \mathrm{L}$. Silica concentrations were $15 \mathrm{mg} / \mathrm{L}$ or less.

Thirty-two samples were collected from Big Spring during water year 1990 for the analyses of nitrogen species, orthophosphorus, and organic carbon. Both the total and dissolved constituents were analyzed. Nitrate- $\mathrm{N}$ generally was the predominant nitrogen species in solution (table 23). Nitrate-N concentrations ranged from 3.0 to $16 \mathrm{mg} / \mathrm{L}$. Ammonia- $\mathrm{N}$ concentrations equaled or exceeded the detection level in about 38 percent of the samples. The maximum ammonia- $\mathrm{N}$ concentration was $1.8 \mathrm{mg} / \mathrm{L}$. Organic-N concentrations ranged from less than 0.10 to 9.1 $\mathrm{mg} / \mathrm{L}$, orthophosphorus concentrations ranged from 0.20 to $1.3 \mathrm{mg} / \mathrm{L}$, and organic-carbon concentrations ranged from less than 0.10 to 36 $\mathrm{mg} / \mathrm{L}$.

Four of seven herbicides analyzed were detected in 32 samples from Big Spring (table 24). Alachlor was detected in 22 percent of the samples; atrazine in 100 percent; cyanazine in 44 percent; and metolachlor in 6 percent. Butylate, metribuzin, and trifluralin were not detected. Alachlor concentrations ranged from less than 0.10 to $0.77 \mu g / L$; atrazine concentrations from 0.14 to $4.5 \mu \mathrm{g} / L$; cyanazine concentrations from less than 0.10 to $0.47 \mu g / L$; and metolachlor concentrations from less than 0.10 to $2.1 \mu \mathrm{g} / L$. 


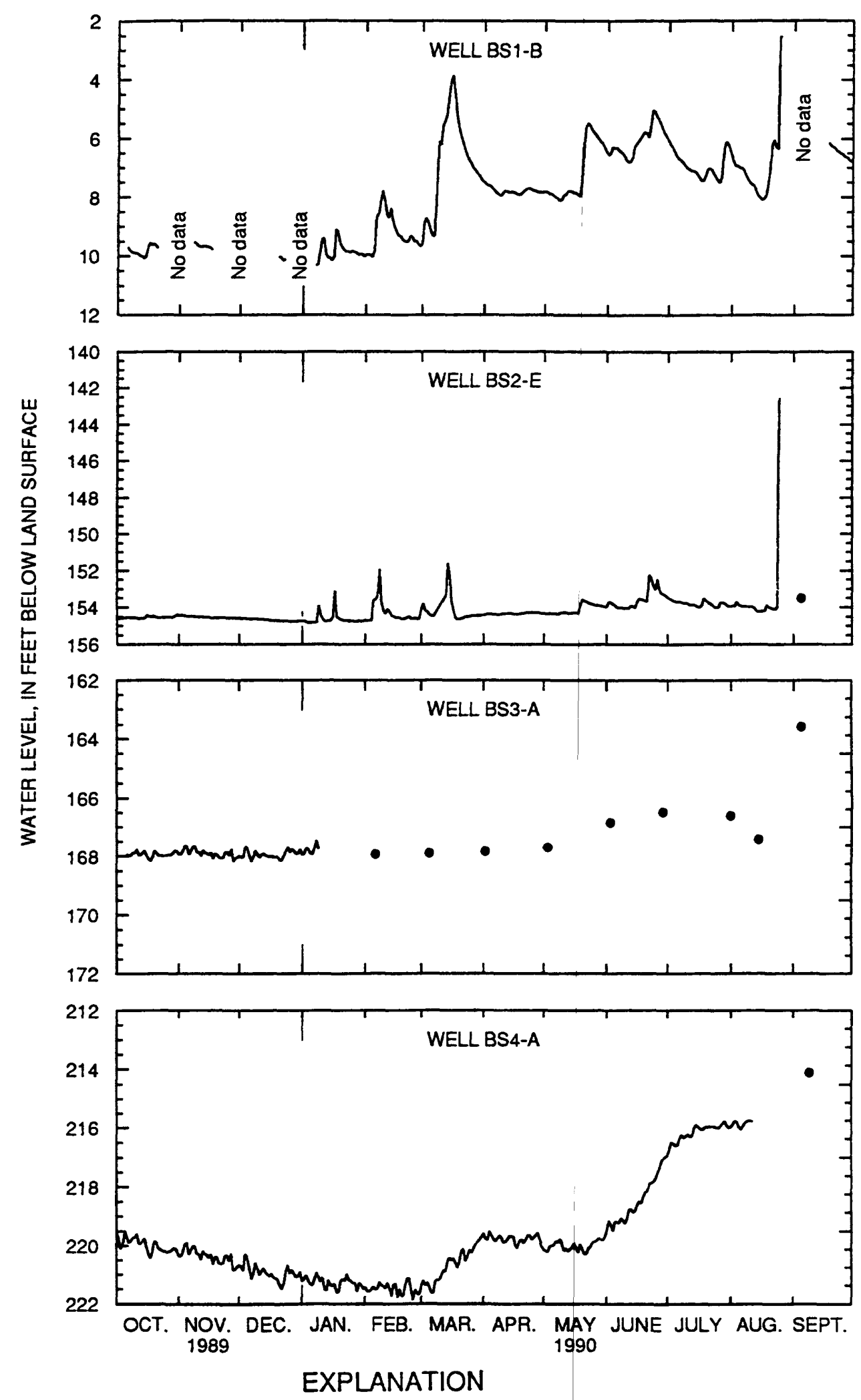

- ISOLATED WATER-LEVEL MEASUREMENT

Figure 13. Daily mean water levels in the Galena aquifer in the Big Spring basin, water year 1990. 

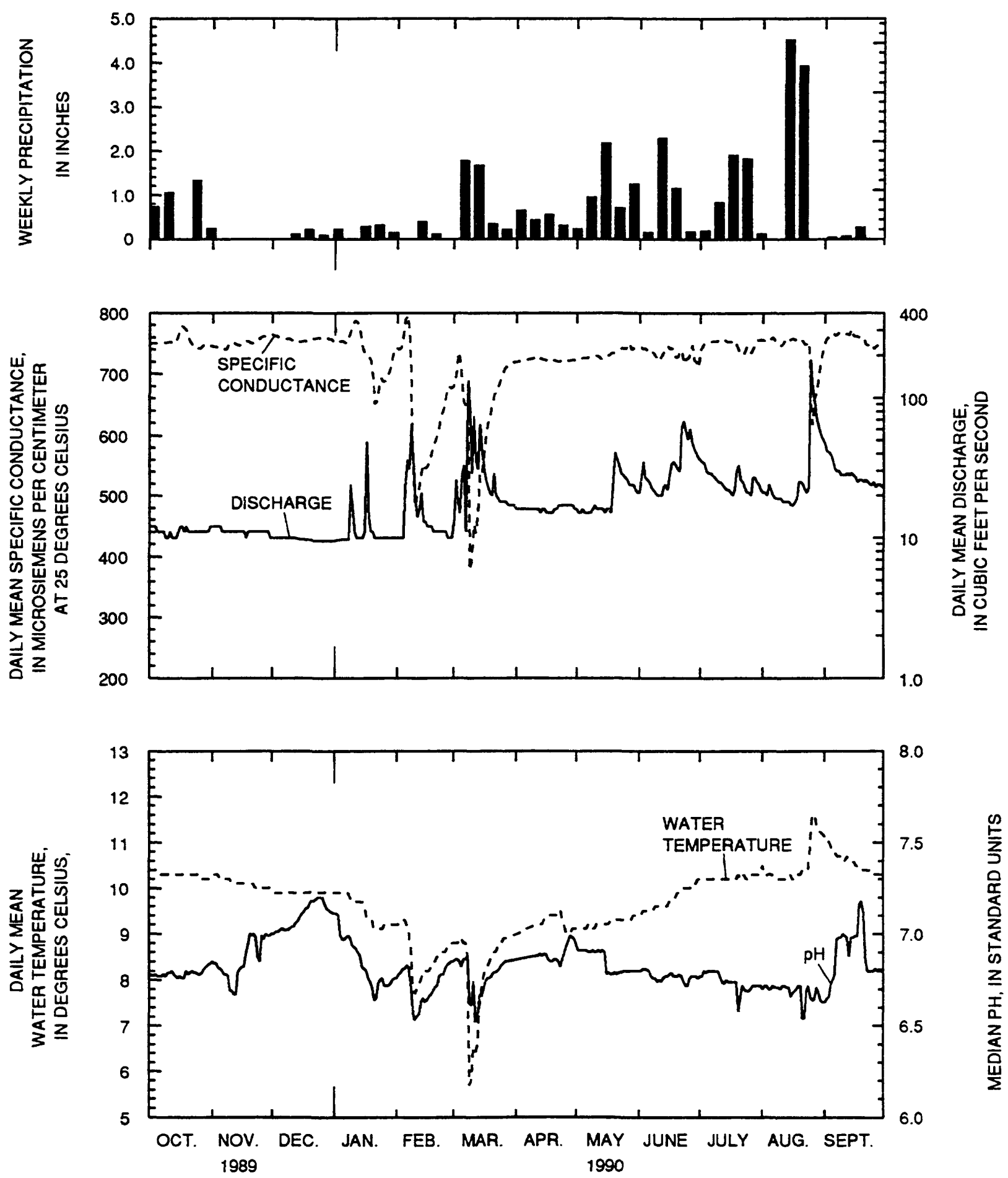

Figure 14. Weekly precipitation, daily mean specific conductance, discharge, water temperature, and daily median pH at Big Spring, water year 1990. 


\section{Saint Peter Aquifer}

The highest water level in well BS2-G was $185.01 \mathrm{ft}$ below land surface on October 11, and the highest water level in well BS4 was 368.45 $\mathrm{ft}$ below land surface on April 28, 1990. The lowest water level in well BS2-G was $186.31 \mathrm{ft}$ below land surface on February 28, and the lowest water level in well BS4 was $371.69 \mathrm{ft}$ below land surface on September 5, 1990. Water levels are shown in figure 15 and are listed in table 29.

\section{Seepage Measurements}

Stream discharge was measured at 39 sites (fig. 16) on May 29 and 30, 1990, to determine areas where streamflow was being lost to the ground-water system through seepage and to determine areas where water was flowing into streams from ground-water discharge. Water samples were collected concurrently to document nutrient and herbicide inflow from ground water and outflow into the underlying aquifer. The results of the data collection are presented in tables 30 and 31 .

\section{Discharge}

Discharge increased in Roberts Creek from $0.35 \mathrm{ft}^{3} / \mathrm{s}$ at site $\mathrm{RC} 13$, the most upstream site, to $5.54 \mathrm{ft}^{3} / \mathrm{s}$ at site $\mathrm{F} 45$, approximately $11 \mathrm{mi}$ downstream (fig. 17). Streamflow in this reach originated partially from two tributaries, West Branch Roberts Creek and Deer Creek, and from industrial discharge in the headwaters. West Branch Roberts Creek (site RC21) contributed $1.07 \mathrm{ft}^{3} / \mathrm{s}$, Deer Creek (site DC2) contributed approximately $0.43 \mathrm{ft}^{3} / \mathrm{s}$, and industrial discharge (site RC26) contributed $0.95 \mathrm{ft}^{3} / \mathrm{s}$.

Discharge increased downstream from 5.54 $\mathrm{ft}^{3} / \mathrm{s}$ at site $\mathrm{F} 45$ to $7.33 \mathrm{ft}^{3} / \mathrm{s}$ at site RC18. Silver Creek joins Roberts Creek in this 5.8-mi reach. The discharge at the first site (SC4) upstream of the mouth of Silver Creek was $1.30 \mathrm{ft}^{3} / \mathrm{s}$. Discharge decreased from $7.33 \mathrm{ft}^{3} / \mathrm{s}$ at site $\mathrm{RC} 18$ to $3.29 \mathrm{ft}^{3} / \mathrm{s}$ at the site (RC2) where Roberts Creek leaves the Big Spring basin. Determination of seepage in Roberts Creek and Silver Creek is complicated by nonuniform point-source discharges in the headwaters of the streams. A municipal wastewater-treatment plant discharges upstream of site $\mathrm{SC10}$, and a creamery discharges wastewater upstream of site SC13. Discharges were 0.42 and $0.95 \mathrm{ft}^{3} / \mathrm{s}$, respectively, at sites SC10 and SC13. Discharge at site L23S on the main stem of Silver Creek was $0.56 \mathrm{ft}^{3} / \mathrm{s}$. Downstream of the confluence of Silver Creek and the East Fork of Silver Creek at site $\mathrm{SC} 1$, the streamflow was $0.70 \mathrm{ft}^{3} / \mathrm{s}$.

The total measured discharge lost from streams in the Big Spring basin during the seepage study was $8.56 \mathrm{ft}^{3} / \mathrm{s}$. This includes a measured loss of $5.1 \mathrm{ft}^{3} / \mathrm{s}$ from Roberts Creek, $1.9 \mathrm{ft}^{3} / \mathrm{s}$ from the Silver Creek subbasin, and $1.55 \mathrm{ft}^{3} / \mathrm{s}$ from Hatchery Creek. The total measured discharge leaving the basin in streams was $3.63 \mathrm{ft}^{3} / \mathrm{s}$.

\section{Water Quality}

Total dissolved-nitrogen concentrations (nitrate-N, ammonia-N, and organic-N) were less than $5.0 \mathrm{mg} / \mathrm{L}$ in samples from 7 sites, were 5.0 to $10 \mathrm{mg} / \mathrm{L}$ in samples from 28 sites, and were greater than $10 \mathrm{mg} / \mathrm{L}$ in samples from 3 sites in the Big Spring basin during the seepage study. The predominant nitrogen species was nitrate. Total dissolved-nitrogen- $\mathrm{N}$ concentrations in water from the main stem of Roberts Creek ranged from $22 \mathrm{mg} / \mathrm{L}$ at site $\mathrm{RC} 13$ to 4.5 $\mathrm{mg} / \mathrm{L}$ at site $\mathrm{RC2}$ (fig. 17) Total dissolved-nitrogen- $\mathrm{N}$ concentrations in water from Silver Creek ranged from 3.7 to $16 \mathrm{mg} / \mathrm{L}$.

Total dissolved-nitrogen loads at sites on the main stem of Roberts Creek ranged from less than 0.01 to 0.14 ton/d. The greatest load $(0.14$ ton/d) was measured at the first site (RC18) downstream of the mouth of Silver Creek. The total measured nitrogen-load lost from streams in the Big Spring basin during the seepage study was 0.29 ton/d. This includes a measured nitrogen-load loss of 0.21 ton/d from Roberts Creek, 0.04 ton/d from the Silver Creek subbasin, and about 0.04 ton/d from Hatchery Creek. The total measured nitrogen load leaving the basin in streams was about $0.04 \mathrm{ton} / \mathrm{d}$.

During the seepage study, samples were collected at 32 sites for the analyses of selected herbicides (table 31). Alachlor was detected in 31 percent of the samples; atrazine in 97 percent; cyanazine in 38 percent; and metolachlor in 19 percent. Alachlor concentrations ranged from less than 0.10 to $0.68 \mu \mathrm{g} / \mathrm{L}$; atrazine ranged from less than 0.10 


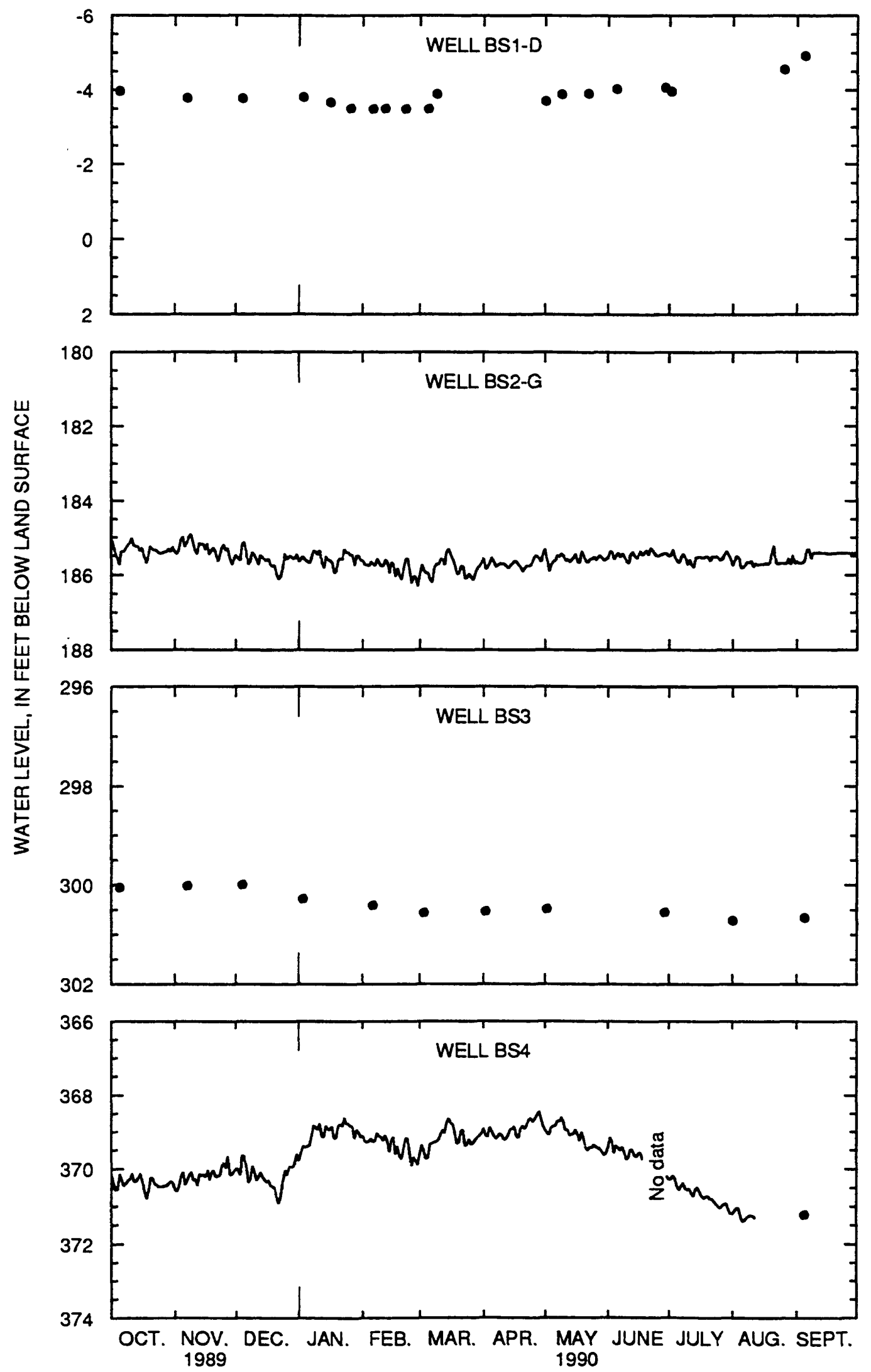

Figure 15. Daily mean water levels in the Saint Peter aquifer in the Big Spring basin, water year 1990. 


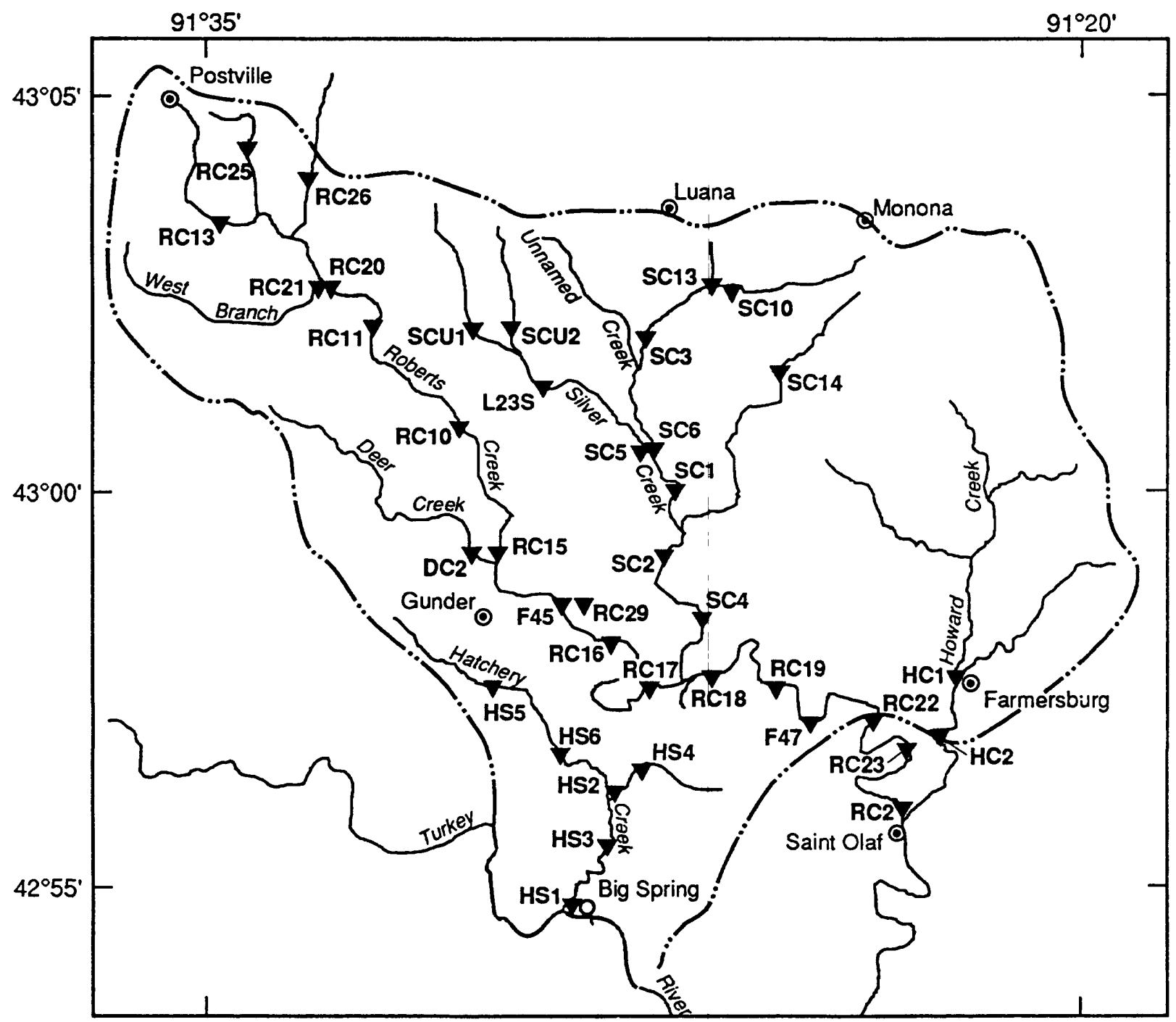

Base from U.S. Geological Survey

Clayton County, 1:100,000, 1985

\section{EXPLANATION}

Big Spring ground-water basin location from Hallberg and others, 1983

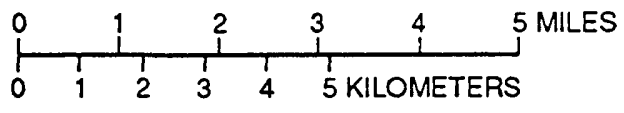

\section{-..- BIG SPRING GROUND-WATER BASIN DIVIDE \\ ${ }^{\text {HS1 }}$ V SEEPAGE-STUDY SAMPLING SITE AND IDENTIFICATION \\ Q SPRING}

Figure 16. Location of seepage-study sampling sites. 


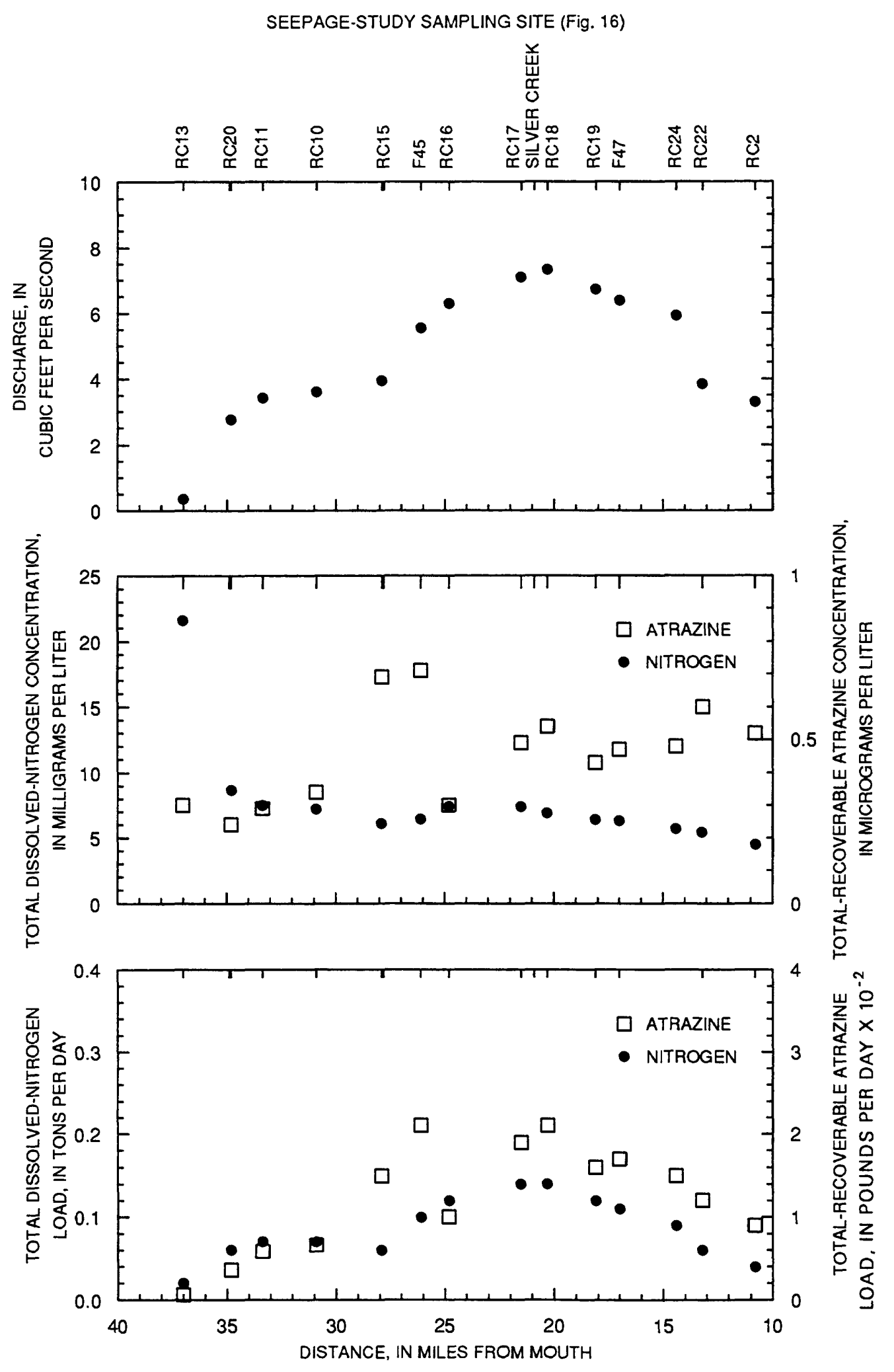

Figure 17. Stream discharge and total dissolved-nitrogen and total-recoverable atrazine concentrations and loads in Roberts Creek, May 29-30, 1990. 
to $1.0 \mu g / L$; cyanazine ranged from less than 0.10 to $0.21 \mu \mathrm{g} / L$; and metolachlor ranged from less than 0.10 to $1.3 \mu \mathrm{g} / L$. Metribuzin was detected in only one sample at $0.23 \mu \mathrm{g} / \mathrm{L}$. Butylate and trifluralin were not detected.

The measured atrazine load in the main stem of Roberts Creek was greatest $(0.021 \mathrm{lb} / \mathrm{d})$ at site RC18, just downstream of the mouth of Silver Creek. Downstream at site RC2, the atrazine load was $0.009 \mathrm{lb} / \mathrm{d}$. The total measured atrazine loss in the main stem of Roberts Creek was about $0.028 \mathrm{lb} / \mathrm{d}$. The total measured atrazine loss in the Big Spring basin during the seepage study was approximately $0.033 \mathrm{lb} / \mathrm{d}$.

\section{Herbicide Degradation Study}

Onsite measurements were made, and water-quality samples were collected at six sites on a losing reach of Roberts Creek (fig. 18) to study the degradation of herbicides in small streams. Data were collected from April through November 1990 during stable flow conditions (tables 32-34). Samples were analyzed for two atrazine metabolites and 10 common herbicides. Using the analyses of atrazine and two of its metabolites, a degradation ratio was calculated. The DDAR (deethylatrazine plus deisopropylatrazine-to-atrazine ratio) was calculated using the following equation.

DDAR = deethylatrazine + deisopropylatrazine/atrazine.

A total of 40 samples were collected at the six sites. Alachlor was detected in 65 percent of the samples; atrazine in 100 percent; cyanazine in about 15 percent; and metolachlor in 52 percent (table 33). Deethylatrazine was detected in all samples, and deisopropylatrazine was detected in 68 percent of the samples. The DDAR ratio was lowest in the June (fig. 19) samples when the atrazine concentrations were the largest. The highest DDAR ratios were in September and November. Prometon was detected in one sample. Propazine and simazine were detected in about 15 percent of the samples (table 34). Ametryn, prometryn, and terbutryn were not detected.

\section{SELECTED REFERENCES}

Buchanan, T.J., and Somers, W.P., 1969, Discharge measurements at gaging stations:
U.S. Geological Survey Techniques of Water-Resources Investigations, Book 3, Chapter A8, 65 p.

Guy, H.P., 1969, Laboratory theory and methods for sediment analysis: U.S. Geological Survey Techniques of Water-Resources Investigations, Book 5, Chapter C1, 58 p.

Guy, H.P., and Norman, V.W., 1970, Field methods for measurement of fluvial sediment: U.S. Geological Survey Techniques of Water-Resources Investigations, Book 3, Chapter C2, 59 p.

Hallberg, G.R., Hoyer, B.E., Bettis, E.A., and Libra, R.D., 1983, Hydrogeology, water quality, and land management in the Big Spring basin, Clayton County, Iowa: Iowa Department of Natural Resources, Geological Survey Bureau Open-File Report 83-3, 191 p.

Hallberg, G.R., Libra, R.D., Bettis, E.A., and Hoyer, B.E., 1984, Hydrogeologic and water quality investigations in the Big Spring basin, Clayton County, Iowa: Iowa Department of Natural Resources, Geological Survey Bureau Open-File Report 84-4, 231 p.

Kalkhoff, S.J., 1989, Hydrologic data for the Big Spring basin, Clayton County, Iowa, water year 1988: U.S. Geological Survey Open-File Report 89-230, 44 p.

Kalkhoff, S.J., and Kuzniar, R.L., 1991, Hydrologic data for the Big Spring basin, Clayton County, Iowa, water year 1989: U.S. Geological Survey Open-File Report 91-63, $66 \mathrm{p}$.

Kennedy, D.J., 1983, Computation of continuous records of streamflow: U.S. Geological Survey Techniques of Water-Resources Investigations, Book 3, Chapter A13, 53 p.

Libra, R.D., Hallberg, G.R., Hoyer, B.E., and Johnson, L.G., 1986, Agricultural impacts on ground water quality--The Big Spring basin study, Iowa: Proceedings of the Agricultural Impacts on Ground Water Conference, p. 253-373. 


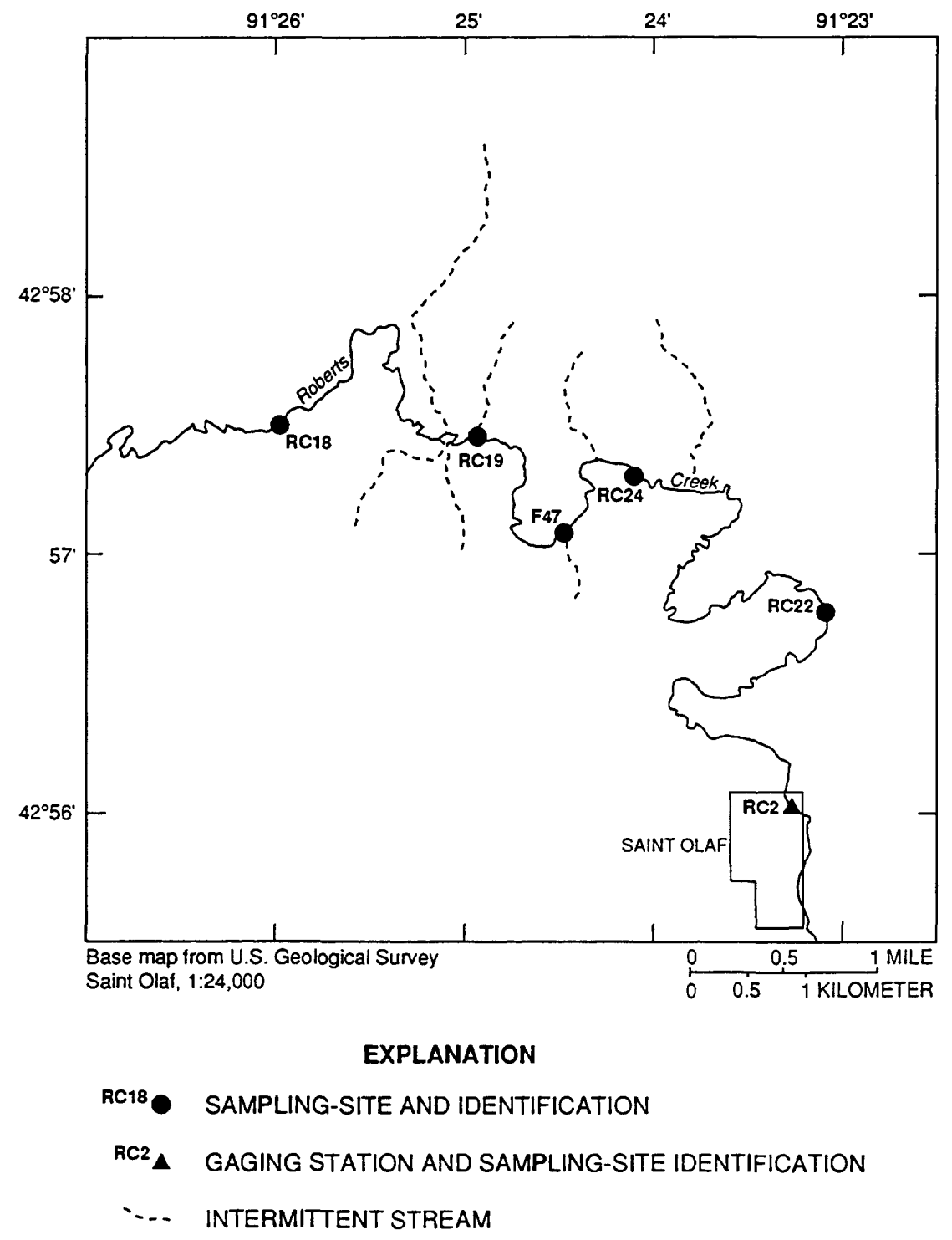

Figure 18. Location of degradation-study sampling sites in a selected reach of Roberts Creek.

National Atmospheric Deposition Program/ National Trends Network, 1988, Instruction manual NADP/NTN site operation: Boulder, Colorado State University Natural Resource Ecology Laboratory.

National Oceanic and Atmospheric Administration, 1990a, Climatological data Iowa August 1990: U.S. Department of Commerce, v. 101, no. 8, 30 p.

1990b, Climatological data Iowa September 1990: U.S. Department of Commerce, v. 101, no. 9, 30 p.

Rantz, S.E., and others, 1982a, Measurement and computation of streamflow--Volume 1. Measurement of stage and discharge: U.S. Geological Survey Water-Supply Paper 2175, p. 1-284.

$1982 \mathrm{~b}$, Measurement and computation of streamflow--Volume 2. Computation of discharge: U.S. Geological Survey Water-Supply Paper 2175, p. 285-631.

Rowden, R.D., and Libra, R.D., 1990, Hydrogeologic observations from bedrock monitoring well nests in the Big Spring basin: Iowa Department of Natural Resources, Geological Survey Bureau Open-File Report 90-1, 27 p. 

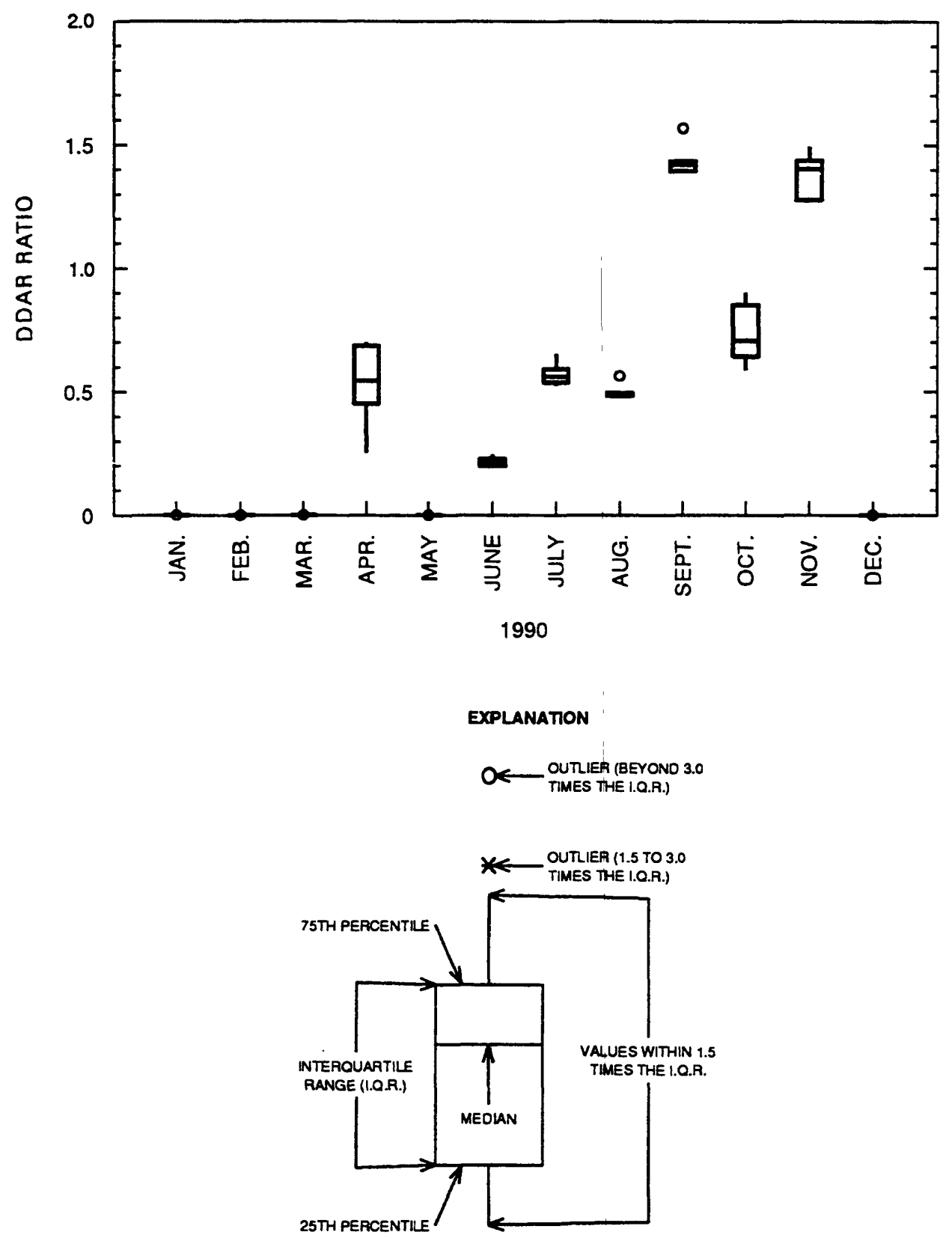

Figure 19. Deethylatrazine plus deisopropylatrazine-to-atrazine ratio (DDAR) in a selected reach of Roberts Creek, April-November 1990.

Thurman, E.M., Meyer, M.T., Pomes, M.L., Perry, C.A., and Schwab, W.P., 1990, Enzyme-linked immunosorbent assay compared with gas chromatography/ mass spectrometry for the determination of triazine herbicides in water: Analytical Chemistry, v. 62, p. 2043-2048.

U.S. Environmental Protection Agency, 1983, Methods for chemical analysis of water and wastes: Cincinnati, Ohio, EPA600/4-79-020.

Wood, W.W., 1976, Guidelines for collection and field analysis of ground-water samples for selected unstable constituents: U.S. Geological Survey Techniques of Water-Resources Investigations, Book 1, Chapter D2, $24 \mathrm{p}$ 


\section{HYDROLOGIC DATA}

HYDROLOGIC DATA 29 
Table 1. Records of monitoring sites in the Big Spring basin, Clayton County, Iowa [Lat., Latitude; Long., Longitude, $\mathbf{m i}^{2}$, square miles]

\begin{tabular}{|c|c|c|c|c|c|c|}
\hline $\begin{array}{l}\text { Site } \\
\text { desig- } \\
\text { nation } \\
\text { number } \\
\text { (fig.2) }\end{array}$ & $\begin{array}{l}\text { Site } \\
\text { identi- } \\
\text { cation } \\
\text { number }\end{array}$ & Site name & $\begin{array}{l}\text { Lat.jati } \\
\text { (degrees, } \\
\text { seconds) }\end{array}$ & $\begin{array}{l}\text { ion } \\
\text { minutes, }\end{array}$ & $\begin{array}{l}\text { Drainage } \\
\text { aregి } \\
\left(\mathrm{mi}^{2}\right)\end{array}$ & Type of $_{\text {record }}$ \\
\hline \multirow{2}{*}{$\begin{array}{l}\text { DC5 } \\
\text { BOOGD } \\
\text { L23S } \\
\text { RC2 } \\
\text { Bis } \\
\text { Spring }\end{array}$} & $\begin{array}{l}430040091325401 \\
05412060 \\
05412070 \\
05412100\end{array}$ & $\begin{array}{l}\text { Deer Creek near Postville } \\
\text { Unnamed Creek near Luana } \\
\text { Silver Creek near Luana } \\
\text { Roberts Creek above Saint olaf }\end{array}$ & $\begin{array}{l}430400 \\
430224 \\
430119 \\
425549\end{array}$ & $\begin{array}{l}0913254 \\
0912807 \\
0912921 \\
0912303\end{array}$ & $\begin{aligned} & 1.1 \\
&-1.15 \\
&- 4.39 \\
& 70.7\end{aligned}$ & $\begin{array}{l}\text { S,QN } \\
\mathbf{S}, P, Q N \\
\text { S } \\
S, P, Q N\end{array}$ \\
\hline & 05411950 & Bis Spring near Elkader & 425433 & 0912801 & $2 i 03$ & $G, P, Q W$ \\
\hline
\end{tabular}
1 S, surface water; G, ground water; P, precipitation; QW, water quality.
2 Ground-water drainage. 
Table 2. Records of selected monitoring wells, lysimeters, and tile line in the Big Spring basin, Clayton County, Iowa

[Sec., section; T., township; R., range; --, no data; well construction data from Iowa Department of Natural Resources, Geological Survey Bureau]

\begin{tabular}{|c|c|c|c|c|c|c|c|c|c|}
\hline $\begin{array}{l}\text { Site } \\
\text { designa- } \\
\text { tion } \\
\text { (fig. 2) }\end{array}$ & $\begin{array}{l}\text { Site } \\
\text { identi- } \\
\text { cation } \\
\text { number }\end{array}$ & $\begin{array}{l}\text { Loc } \\
\text { Sec. }\end{array}$ & $\begin{array}{c}\text { ation } \\
\mathrm{T} \text {. }\end{array}$ & R. & $\begin{array}{l}\text { Alti- } \\
\text { tude } \\
\text { (feet } \\
\text { above } \\
\text { sea } \\
\text { level }\end{array}$ & $\begin{array}{l}\text { Well } \\
\text { depth } \\
\text { ( feet } \\
\text { below } \\
\text { land } \\
\text { surface) }\end{array}$ & $\begin{array}{l}\text { Open } \\
\text { interval } \\
\text { (feet } \\
\text { below } \\
\text { land } \\
\text { ) surface) }\end{array}$ & $\begin{array}{c}\text { Aquifer } \\
-\end{array}$ & $\begin{array}{c}\text { Frequency of } \\
\text { measurement }\end{array}$ \\
\hline \multicolumn{10}{|c|}{ We11 nest BS1 } \\
\hline $\begin{array}{l}B S 1-A \\
B S 1-B \\
B S 1-D\end{array}$ & $\begin{array}{l}425433091285001 \\
425433091285002 \\
425433091285004\end{array}$ & $\begin{array}{l}\text { NWSE31 } \\
\text { NWSE31 } \\
\text { NWSE31 }\end{array}$ & $\begin{array}{l}94 N \\
94 N \\
94 N\end{array}$ & $\begin{array}{l}05 W \\
05 W \\
05 W\end{array}$ & $\begin{array}{l}855 \\
855 \\
855\end{array}$ & $\begin{array}{r}36 \\
85 \\
215\end{array}$ & $\begin{array}{c}33-36 \\
61-85 \\
173-215\end{array}$ & $\begin{array}{l}\text { Unconsolidated } \\
\text { Galena } \\
\text { Saint Peter }\end{array}$ & $\begin{array}{l}\text { Continuous } \\
\text { Continuous } \\
\text { Monthiy }\end{array}$ \\
\hline $\begin{array}{l}\text { BS2-A } \\
\text { BS2-B } \\
\text { BS2-C } \\
\text { BS2-D } \\
\text { BS2-E } \\
\text { BS2-F } \\
\text { BS2-G }\end{array}$ & $\begin{array}{c}\overline{-} \\
-- \\
-- \\
-- \\
425736091260302 \\
425736091260303\end{array}$ & $\begin{array}{l}\text { SENW16 } \\
\text { SENW16 } \\
\text { SENW16 } \\
\text { SENW16 } \\
\text { SENW16 } \\
\text { SENW16 } \\
\text { SENW16 }\end{array}$ & $\begin{array}{l}94 N \\
94 N \\
94 N \\
94 N \\
94 N \\
94 N \\
94 N\end{array}$ & $\begin{array}{l}05 W \\
05 W \\
05 W \\
05 W \\
05 W \\
05 W \\
05 W\end{array}$ & $\begin{array}{l}\text { We11 nest } \\
950 \\
950 \\
950 \\
950 \\
950 \\
950 \\
950\end{array}$ & $\begin{array}{r}\text { BS2 } \\
60 \\
127 \\
128 \\
151 \\
180 \\
286 \\
335\end{array}$ & $\begin{array}{l}50-60 \\
122-127 \\
118-128 \\
134-151 \\
165-180 \\
272-286 \\
300-335\end{array}$ & $\begin{array}{l}\text { Galena } \\
\text { Galena } \\
\text { Galena } \\
\text { Galena } \\
\text { Galena } \\
\text { Saint Peter }\end{array}$ & $\begin{array}{l}\text { Intermittent } \\
\text { Intermittent } \\
\text { Intermittent } \\
\text { Intermittent } \\
\text { Continuous } \\
\text { Intermittent } \\
\text { Continuous }\end{array}$ \\
\hline \multirow[b]{2}{*}{$\begin{array}{l}\text { BS3 } \\
\text { BS3-A } \\
\text { BS3-B } \\
\text { BS3-C }\end{array}$} & & & & $\cdot$ & \multicolumn{3}{|c|}{ We1l nest BS3 } & & \\
\hline & $\begin{array}{l}430145091253001 \\
430145091253002 \\
430145091253003 \\
430145091253004\end{array}$ & $\begin{array}{l}\text { SWNW22 } \\
\text { SWNW22 } \\
\text { SWNW22 } \\
\text { SWNW22 }\end{array}$ & $\begin{array}{l}95 N \\
95 N \\
95 N \\
95 N\end{array}$ & $\begin{array}{l}05 W \\
05 W \\
05 W \\
05 W\end{array}$ & $\begin{array}{l}1,080 \\
1,080 \\
1,080 \\
1,080\end{array}$ & $\begin{array}{r}397 \\
185 \\
60 \\
26\end{array}$ & $\begin{array}{c}351-397 \\
165-185 \\
-- \\
11-26\end{array}$ & $\begin{array}{l}\text { Saint Peter } \\
\text { Galena } \\
\text { Galena } \\
\text { Unconsolidated }\end{array}$ & $\begin{array}{l}\text { Monthly } \\
\text { Continuous } \\
\text { Intermittent } \\
\text { Continuous }\end{array}$ \\
\hline \multicolumn{10}{|c|}{ We11 nest BS4 } \\
\hline $\begin{array}{l}\text { BS4 } \\
\text { BS4-A } \\
\text { BS4-B } \\
\text { BS4-C }\end{array}$ & $\begin{array}{l}430133091344801 \\
430133091344802 \\
430133091344803 \\
430133091344804\end{array}$ & $\begin{array}{l}\text { NWSE20 } \\
\text { NWSE20 } \\
\text { NWSE20 } \\
\text { NWSE20 }\end{array}$ & $\begin{array}{l}95 N \\
95 N \\
95 N \\
95 N\end{array}$ & $\begin{array}{l}06 W \\
06 W \\
06 W \\
06 W\end{array}$ & $\begin{array}{l}1,160 \\
1,160 \\
1,160 \\
1,160\end{array}$ & $\begin{array}{r}580 \\
361 \\
139 \\
61\end{array}$ & $\begin{array}{l}550-580 \\
261-361 \\
130-139 \\
50-61\end{array}$ & $\begin{array}{l}\text { Saint Peter } \\
\text { Galena } \\
\text { Unconsolidated } \\
\text { Unconsolidated }\end{array}$ & $\begin{array}{l}\text { Continuous } \\
\text { Continuous } \\
\text { Continuous } \\
\text { Continuous }\end{array}$ \\
\hline \multicolumn{10}{|c|}{ Single wells } \\
\hline $\begin{array}{l}\text { DCW1 } \\
\text { DCW2 } \\
\text { DCW3 }\end{array}$ & $\begin{array}{l}430040091325402 \\
430040091325403 \\
430040091325410\end{array}$ & $\begin{array}{l}\text { SESE28 } \\
\text { SESE28 } \\
\text { SESE28 }\end{array}$ & $\begin{array}{l}95 N \\
95 N \\
95 N\end{array}$ & $\begin{array}{l}06 W \\
06 W \\
06 W\end{array}$ & $\begin{array}{l}1,070 \\
1,070 \\
1,100\end{array}$ & $\begin{array}{l}13 \\
11 \\
15\end{array}$ & $\begin{array}{r}11-13 \\
8-11 \\
13-15\end{array}$ & $\begin{array}{l}\text { Unconsolidated } \\
\text { Unconsolidated } \\
\text { Unconsolidated }\end{array}$ & $\begin{array}{l}\text { Continuous } \\
\text { Monthiy } \\
\text { Monthly }\end{array}$ \\
\hline \multicolumn{10}{|c|}{ Lysimeters } \\
\hline $\begin{array}{l}\text { DCLA } \\
\text { DCLB } \\
\text { DCLC } \\
\text { DCLD }\end{array}$ & $\begin{array}{l}430040091325404 \\
430040091325405 \\
430040091325406 \\
430040091325407\end{array}$ & $\begin{array}{l}\text { SESE28 } \\
\text { SESE28 } \\
\text { SESE28 } \\
\text { SESE28 }\end{array}$ & $\begin{array}{l}95 N \\
95 N \\
95 N \\
95 N\end{array}$ & $\begin{array}{l}06 W \\
06 W \\
06 W \\
06 W\end{array}$ & $\begin{array}{l}1,070 \\
1,070 \\
1,070 \\
1,070\end{array}$ & $\begin{array}{l}8.5 \\
7.0 \\
5.5 \\
4.0\end{array}$ & $\begin{array}{l}-- \\
-- \\
-- \\
--\end{array}$ & $\begin{array}{l}\text { Unconsolidated } \\
\text { Unconsolidated } \\
\text { Unconsolidated } \\
\text { Unconsolidated }\end{array}$ & $\begin{array}{l}\text { Monthly } \\
\text { Monthly } \\
\text { Monthly } \\
\text { Monthly }\end{array}$ \\
\hline \multicolumn{10}{|c|}{ Tile line } \\
\hline DCT2 & 430040091325408 & SESE28 & 95N & $06 W$ & 1,070 & -- & -- & -- & Monthly \\
\hline
\end{tabular}


Table 3. Location and drainage area of seepage-study sampling sites, Clayton County, Iowa

[Lat., latitude; Long., longitude; $\mathrm{mi}^{2}$, square miles; --, no data]

\begin{tabular}{|c|c|c|c|c|c|}
\hline \multirow{2}{*}{$\begin{array}{l}\text { Site } \\
\text { desig- } \\
\text { nation } \\
\text { (fig. } \\
\text { HS5 } \\
\text { BS6 } \\
\text { BS4 } \\
\text { BS2 } \\
\text { BS3 }\end{array}$} & $\begin{array}{l}\text { Site } \\
-\begin{array}{l}\text { Sidenti- } \\
\text { idetion } \\
\text { cation } \\
\text { number }\end{array} \\
\text { 16) }\end{array}$ & Site name & \multicolumn{2}{|c|}{$\begin{array}{l}\text { Location } \\
\text { Lat. Long. } \\
\text { (minutes, degrees } \\
\text { seconds) }\end{array}$} & \multirow{2}{*}{$\begin{array}{c}\begin{array}{c}\text { Drainage } \\
\text { areg } \\
\text { (mi }\end{array} \\
1.28 \\
2.84 \\
1.36 \\
1.85 \\
7.02\end{array}$} \\
\hline & $\begin{array}{l}-- \\
-- \\
-- \\
--\end{array}$ & $\begin{array}{l}\text { Hatchery Creek near Gunder } \\
\text { Batchery Creek southeast of Gunder } \\
\text { Batchery Creek tributary north of Big Spring } \\
\text { Batchery Creek tributary near Bis Spring } \\
\text { Batchery Creek near Big Spring }\end{array}$ & $\begin{array}{l}425734 \\
425647 \\
425629 \\
425606 \\
425536\end{array}$ & $\begin{array}{l}0913012 \\
0912859 \\
0912737 \\
0912806 \\
0912806\end{array}$ & \\
\hline $\begin{array}{l}\mathrm{BS} 1 \\
\mathrm{RC} 13 \\
\mathrm{RC25} \\
\mathrm{RC26} \\
\mathrm{RC} 21\end{array}$ & $\begin{array}{l}-- \\
\overline{--} \\
\overline{--}\end{array}$ & $\begin{array}{l}\text { Batchery Creek at Bis Spring } \\
\text { Roberts Creek tributary near Postville } \\
\text { Roberts Creek at Postvilie } \\
\text { Roberts Creek tributary at Bwy } 52 \\
\text { West Branch Roberts Creek at mouth }\end{array}$ & $\begin{array}{l}425446 \\
430327 \\
430419 \\
430409 \\
430244\end{array}$ & $\begin{array}{l}0912853 \\
0913440 \\
0913413 \\
0913312 \\
0913300\end{array}$ & $\begin{array}{l}8.80 \\
2.28 \\
--- \\
--14\end{array}$ \\
\hline $\begin{array}{l}\mathrm{RC} 20 \\
\mathrm{RC} 11 \\
\mathrm{RC} 10 \\
\mathrm{RC} 15 \\
\mathrm{DC} 2\end{array}$ & $\begin{array}{l}-- \\
=- \\
=- \\
--\end{array}$ & $\begin{array}{l}\text { Roberts Creek near Postville } \\
\text { Roberts Creek southeast of Postville } \\
\text { Roberts Creek near Luana } \\
\text { Roberts Creek at Gunder } \\
\text { Deer Creek at Gunder }\end{array}$ & $\begin{array}{l}430240 \\
430211 \\
430057 \\
425908 \\
425908\end{array}$ & $\begin{array}{l}0913253 \\
0913216 \\
0913042 \\
0913002 \\
0913025\end{array}$ & $\begin{array}{r}11.1 \\
13.2 \\
15.9 \\
18.2 \\
5.56\end{array}$ \\
\hline $\begin{array}{l}\text { F45 } \\
\text { RC29 } \\
\text { RC16 } \\
\text { RC17 } \\
\text { SC10 }\end{array}$ & $\begin{array}{l}-- \\
\overline{--} \\
=- \\
--\end{array}$ & $\begin{array}{l}\text { Roberts Creek east of Gunder } \\
\text { Roberts Creek tributary } 2 \text { East of Gunder } \\
\text { Roberts Creek north of Big Spring } \\
\text { Roberts Creek near Big Spring } \\
\text { East Fork Silver Creek near Monona }\end{array}$ & $\begin{array}{l}425830 \\
425830 \\
425806 \\
425735 \\
430240\end{array}$ & $\begin{array}{l}0912858 \\
0912837 \\
0912805 \\
0912722 \\
0912620\end{array}$ & $\begin{array}{l}26.0 \\
28.8 \\
30.4 \\
3.05\end{array}$ \\
\hline $\begin{array}{l}\text { SC13 } \\
\text { SC3 } \\
\text { SC6 } \\
\text { SCU1 } \\
\text { SCU2 }\end{array}$ & $\begin{array}{l}=- \\
=- \\
=- \\
--\end{array}$ & $\begin{array}{l}\text { East Fork Silver Creek tributary near Monona } \\
\text { East Fork Silver Creek near Luana. } \\
\text { East Fork Silver Creek south of Luana } \\
\text { Silver Creek southwest of Luana } \\
\text { Silver Creek tributary southwest of Luena }\end{array}$ & $\begin{array}{l}430240 \\
430203 \\
430054 \\
430210 \\
430201\end{array}$ & $\begin{array}{l}0912606 \\
0912730 \\
0912730 \\
0913033 \\
0912949\end{array}$ & $\begin{array}{r}.28 \\
4.28 \\
9.46 \\
1.36 \\
.70\end{array}$ \\
\hline $\begin{array}{l}\text { L23S } \\
\text { SC5 } \\
\text { SC1 } \\
\text { SC14 } \\
\text { SC2 }\end{array}$ & $\begin{array}{c}05412070 \\
-- \\
-- \\
--\end{array}$ & $\begin{array}{l}\text { Silver Creek near Luana } \\
\text { Silver Creek South of Luana } \\
\text { Silver Creek northeast of Gunder } \\
\text { Silver Creek tributary near Monona } \\
\text { Silver Creek near Gunder }\end{array}$ & $\begin{array}{l}430119 \\
430049 \\
430002 \\
430140 \\
425916\end{array}$ & $\begin{array}{l}0912921 \\
0912744 \\
0912653 \\
0912510 \\
0912712\end{array}$ & $\begin{array}{r}4.39 \\
5.59 \\
17.3 \\
1.13 \\
25.2\end{array}$ \\
\hline $\begin{array}{l}\text { SC4 } \\
\operatorname{RC} 18 \\
\operatorname{RC} 19 \\
\text { F47 } \\
\operatorname{RC} 24\end{array}$ & $\begin{array}{l}-- \\
\overline{--} \\
= \\
--\end{array}$ & $\begin{array}{l}\text { Silver Creek East of Gunder } \\
\text { Roberts Creek northeast of Big Spring } \\
\text { Roberts Creek northwest of Saint Olaf } \\
\text { Roberts Creek west of Farmersburg } \\
\text { Roberts Creek near Farmersburg }\end{array}$ & $\begin{array}{l}425824 \\
425736 \\
425733 \\
425706 \\
425724\end{array}$ & $\begin{array}{l}0912630 \\
0912603 \\
0912510 \\
0912434 \\
0912358\end{array}$ & $\begin{array}{l}28.8 \\
61.8 \\
63.6 \\
64.3 \\
65.2\end{array}$ \\
\hline $\begin{array}{l}\mathrm{RC22} \\
\mathrm{RC2} \\
\mathrm{BC1} \\
\mathrm{BC2} 2\end{array}$ & $054 \overline{12100}$ & $\begin{array}{l}\text { Roberts Creek southwest of Farmersburg } \\
\text { Roberts Creek above Saint olaf } \\
\text { Howard Creek at Farmersburg } \\
\text { Howard Creek near Farmersburg }\end{array}$ & $\begin{array}{l}425641 \\
425549 \\
425744 \\
425648\end{array}$ & $\begin{array}{l}0912226 \\
0912303 \\
0912209 \\
0912223\end{array}$ & $\begin{array}{l}66.6 \\
70.7 \\
13.8 \\
17.8\end{array}$ \\
\hline
\end{tabular}


Table 4. Sample preparation and analytical methods

[EPA methods from U.S. Environmental Protection Agency (1983). mg/L, milligrams per liter; $\mu \mathrm{g} /$, microgram per liter; --, no data]

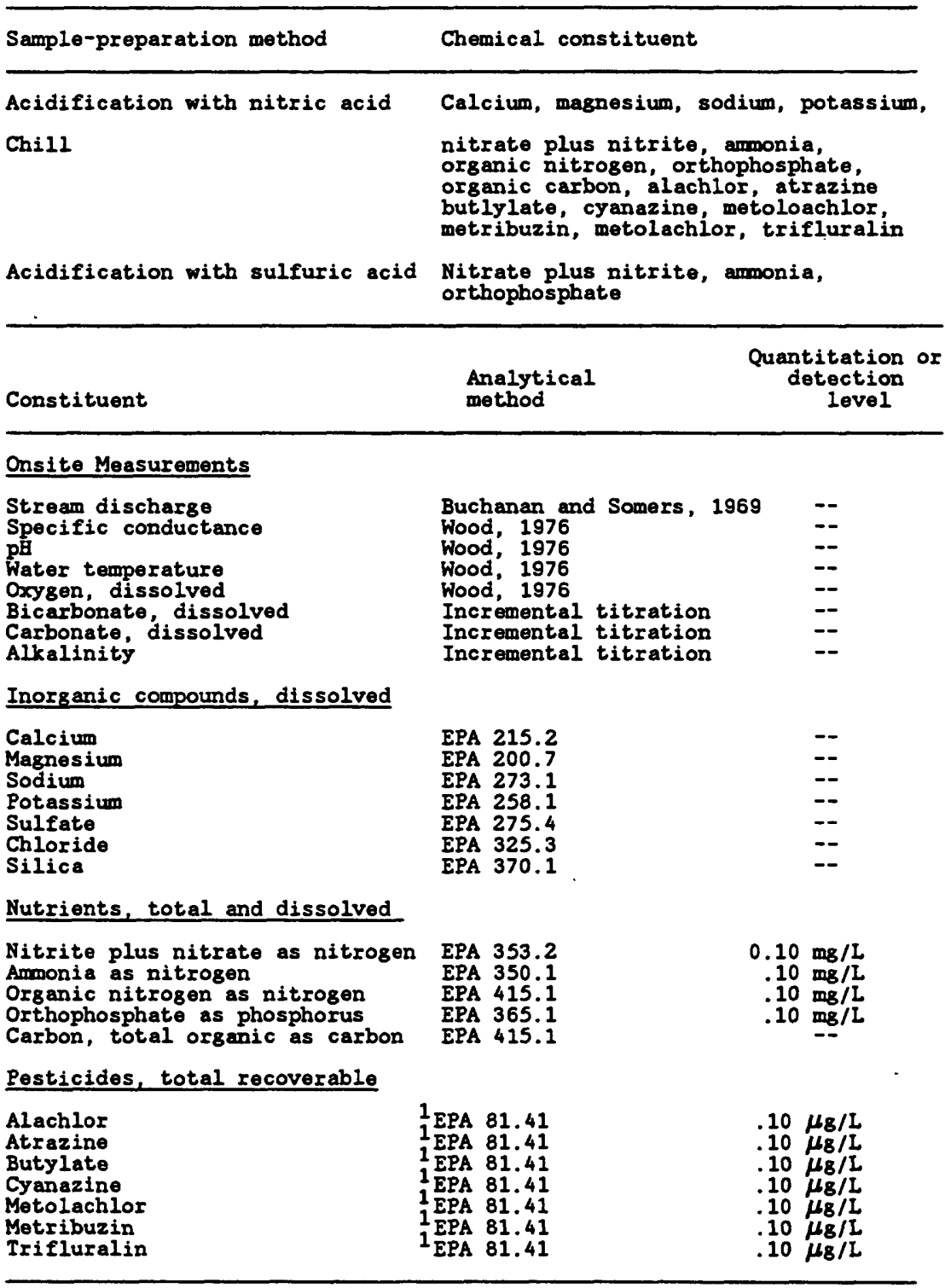

${ }^{1}$ Modified for use of dual-capillary colums. 
Table 5. Statistical summary of precipitation quantity and quality at Big Spring, water year 1990

[Constituents in milligrams per liter unless noted otherwise; $\mu \mathrm{S} / \mathrm{cm}$, microsiemens per centimeter at 25 degrees Celsius; $N$, nitrogen; <, less than detection level indicated]

\begin{tabular}{|c|c|c|c|c|}
\hline Constituent & $\begin{array}{l}\text { Number of } \\
\text { measurable } \\
\text { weekly } \\
\text { samples }\end{array}$ & $\frac{\text { Quantity }}{\text { Median }}$ & $\frac{\text { or concent }}{\text { Minimum }}$ & $\frac{\text { ation }}{\text { Maximum }}$ \\
\hline $\begin{array}{l}\text { Precipitation (inches) } \\
\text { pH (standard units) } \\
\text { Specific conductance ( } \mu \text { S/ } \\
\text { Calcium } \\
\text { Magnesium }\end{array}$ & $\begin{array}{r}48 \\
34 \\
\text { /cm } 34 \\
38 \\
38\end{array}$ & $\begin{array}{r}0.26 \\
5.89 \\
13 \\
.48 \\
.08\end{array}$ & $\begin{array}{l}0 \\
4.19 \\
5.8 \\
.09 \\
.01\end{array}$ & $\begin{array}{r}4.53 \\
6.98 \\
40 \\
5.3 \\
1.0\end{array}$ \\
\hline $\begin{array}{l}\text { Sodium } \\
\text { Potassium } \\
\text { Sulfate } \\
\text { Chloride } \\
\text { Nitrate as } \mathrm{N} \\
\text { Ammonia as } \mathrm{N}\end{array}$ & $\begin{array}{l}38 \\
38 \\
38 \\
38 \\
41 \\
41\end{array}$ & $\begin{array}{l}.08 \\
.03 \\
1.7 \\
.14 \\
.35 \\
.48\end{array}$ & $\begin{array}{l}.01 \\
.004 \\
.46 \\
.06 \\
<.01 \\
<.01\end{array}$ & $\begin{array}{l}.75 \\
.36 \\
6.8 \\
.52 \\
1.2 \\
2.2\end{array}$ \\
\hline
\end{tabular}


Table 6. Accumulated daily precipitation, water year 1990

[---, missing data]

\begin{tabular}{|c|c|c|c|c|c|c|c|c|c|c|c|c|}
\hline Day & Oct. & Nov. & Dec. & Jan. & Feb. & Mar. & Apr. & May & June & July & Aug. & Sept \\
\hline \multicolumn{13}{|c|}{ Unnamed Creek at Luana (site BOOGD, fig. 2) } \\
\hline $\begin{array}{l}1 \\
2 \\
3 \\
4 \\
5\end{array}$ & $\begin{array}{l}0 \\
0 \\
0 \\
0 \\
.89\end{array}$ & $\begin{array}{l}0 \\
0 \\
0 \\
0 \\
.05\end{array}$ & $\begin{array}{l}0 \\
0 \\
0 \\
0 \\
0\end{array}$ & $\begin{array}{l}0 \\
0 \\
0 \\
0 \\
0\end{array}$ & $\begin{array}{l}0 \\
.01 \\
.04 \\
0 \\
0\end{array}$ & $\begin{array}{l}0 \\
0 \\
0 \\
0 \\
0\end{array}$ & $\begin{array}{l}.02 \\
.22 \\
0 \\
0 \\
0\end{array}$ & $\begin{array}{l}0 \\
0 \\
0 \\
0^{.30}\end{array}$ & $\begin{array}{l}0 \\
1.08 \\
0 \\
0 \\
.14\end{array}$ & $\begin{array}{l}0 \\
0 \\
0 \\
.06 \\
.04\end{array}$ & $\begin{array}{l}--- \\
--- \\
--- \\
---\end{array}$ & $\begin{array}{l}--- \\
=-- \\
--- \\
---\end{array}$ \\
\hline $\begin{array}{r}6 \\
7 \\
8 \\
9 \\
10\end{array}$ & $\begin{array}{l}0 \\
0 \\
0 \\
0^{.04}\end{array}$ & $0_{0}^{.08} .09$ & $\begin{array}{l}0 \\
0 \\
0 \\
0 \\
0\end{array}$ & $\begin{array}{l}0 \\
0 \\
.07 \\
.09\end{array}$ & $\begin{array}{l}0 \\
0 \\
0 \\
0 \\
0\end{array}$ & $\begin{array}{l}0 \\
0 \\
.08 \\
. .66\end{array}$ & $\begin{array}{l}0 \\
0 \\
0 \\
0 \\
.71\end{array}$ & $\begin{array}{l}0 \\
0 \\
0 \\
1.11 \\
.08\end{array}$ & $\begin{array}{l}.01 \\
.09 \\
.01 \\
0_{0}^{.01}\end{array}$ & $\begin{array}{l}0_{0}^{0} .07 \\
0_{0} \\
.09\end{array}$ & $\begin{array}{l}--- \\
--- \\
--- \\
---\end{array}$ & $\begin{array}{l}--- \\
--- \\
--- \\
---\end{array}$ \\
\hline $\begin{array}{l}11 \\
12 \\
13 \\
14 \\
15\end{array}$ & $\begin{array}{l}0 \\
0 \\
0 \\
0 \\
.32\end{array}$ & $\begin{array}{l}0 \\
0 \\
0 \\
0 \\
0\end{array}$ & $\begin{array}{l}0 \\
0 \\
0 \\
0 \\
0\end{array}$ & $\begin{array}{l}0 \\
0 \\
0 \\
0 \\
0\end{array}$ & $\begin{array}{l}0 \\
0 \\
0 \\
0 \\
.03\end{array}$ & $\begin{array}{r}.57 \\
.14 \\
1.52 \\
. .54 \\
0\end{array}$ & $\begin{array}{l}.62 \\
.11 \\
.06 \\
.01 \\
.13\end{array}$ & $\begin{array}{l}.03 \\
0_{0}^{.02} \\
0 \\
.35\end{array}$ & $\begin{array}{r}.03 \\
.87 \\
. .44 \\
0.62\end{array}$ & $\begin{array}{l}.15 \\
0_{0}^{.35} \\
0^{.03}\end{array}$ & $\begin{array}{l}--- \\
--- \\
--\overline{-} \\
---\end{array}$ & $\begin{array}{l}--- \\
=- \\
--- \\
--- \\
---\end{array}$ \\
\hline $\begin{array}{l}16 \\
17 \\
18 \\
19 \\
20\end{array}$ & $\begin{array}{l}0^{.39} \\
0 \\
0 \\
0\end{array}$ & $\begin{array}{l}0 \\
0 \\
0 \\
0 \\
0\end{array}$ & $\begin{array}{l}0 \\
0 \\
0 \\
0^{.15}\end{array}$ & $\begin{array}{l}.03 \\
0^{.04} \\
0 \\
.07\end{array}$ & $\begin{array}{l}0^{.10} \\
0 \\
0 \\
0\end{array}$ & $\begin{array}{l}0 \\
0 \\
0 \\
0 \\
.18\end{array}$ & $\begin{array}{l}0 \\
0 \\
.21 \\
.16 \\
.01\end{array}$ & $\begin{array}{l}0^{.04} \\
0 \\
1.68 \\
0\end{array}$ & $0_{0}^{.45}$ & $\begin{array}{l}0 \\
0 \\
.96 \\
1.47 \\
.04\end{array}$ & $\begin{array}{l}--- \\
--- \\
--- \\
--- \\
--\end{array}$ & $\begin{array}{l}--- \\
=- \\
--- \\
---\end{array}$ \\
\hline $\begin{array}{l}21 \\
22 \\
23 \\
24 \\
25\end{array}$ & $\begin{array}{l}0 \\
0 \\
0 \\
0 \\
0\end{array}$ & $\begin{array}{l}0 \\
0 \\
0 \\
0 \\
0\end{array}$ & $\begin{array}{l}0 \\
0 \\
0 \\
0 \\
.01\end{array}$ & $\begin{array}{l}0 \\
0 \\
0_{0}^{.03}\end{array}$ & $\begin{array}{l}0 \\
0 \\
0_{0}^{.05}\end{array}$ & $\begin{array}{l}0 \\
0^{.26} \\
0 \\
0\end{array}$ & $0_{0}^{.01}$ & $\begin{array}{l}0 \\
0 \\
0 \\
0 \\
\end{array}$ & $\begin{array}{l}.03 \\
1.34 \\
0 \\
0 \\
0\end{array}$ & $\begin{array}{l}0 \\
0 \\
0 \\
0^{.01}\end{array}$ & $\begin{array}{l}--- \\
--- \\
--- \\
--- \\
\end{array}$ & $\begin{array}{l}--- \\
=-- \\
--- \\
---\end{array}$ \\
\hline $\begin{array}{l}26 \\
27 \\
28 \\
29 \\
30 \\
31\end{array}$ & $\begin{array}{l}0 \\
.02 \\
.17 \\
.17 \\
.61 \\
.01\end{array}$ & $\begin{array}{l}0 \\
0 \\
0 \\
0 \\
0\end{array}$ & $\begin{array}{l}0 \\
0_{0}^{.01} \\
0\end{array}$ & $\begin{array}{l}0 \\
0 \\
0 \\
0 \\
0 \\
0\end{array}$ & $\begin{array}{l}0 \\
0 \\
0\end{array}$ & $\begin{array}{l}0 \\
0 \\
0 \\
0 \\
0 \\
0\end{array}$ & $0_{0}^{.07} 0_{0}^{.19}$ & $\begin{array}{l}0 \\
.04 \\
0^{.42} \\
0 \\
0\end{array}$ & $\begin{array}{l}.41 \\
.07 \\
.04 \\
.09 \\
.01\end{array}$ & $\begin{array}{l}0^{.46} \\
0 \\
0 \\
0 \\
0\end{array}$ & $\begin{array}{l}--- \\
=-- \\
--- \\
---\end{array}$ & $\begin{array}{l}=- \\
=- \\
=- \\
---\end{array}$ \\
\hline
\end{tabular}

measured 2.62

.40

.17

.33

.23

$3.95 \quad 2.58$

4.07

6.45

3.73

Roberts Creek above Saint Olaf (site RC2, fig. 2)

\begin{tabular}{|c|c|c|c|c|c|c|c|c|c|c|c|c|}
\hline $\begin{array}{l}1 \\
2 \\
3 \\
4 \\
5\end{array}$ & $\begin{array}{l}0 \\
0 \\
0 \\
0 \\
.67\end{array}$ & $\begin{array}{l}0 \\
0 \\
0 \\
0 \\
.05\end{array}$ & $\begin{array}{l}0 \\
0 \\
0 \\
0 \\
0\end{array}$ & $\begin{array}{l}0 \\
0 \\
0 \\
0.01 \\
0.0\end{array}$ & $\begin{array}{l}0 \\
.01 \\
0.06 \\
0\end{array}$ & $\begin{array}{l}0 \\
0 \\
0 \\
0 \\
0\end{array}$ & $\begin{array}{l}.01 \\
0^{.31} \\
0 \\
0\end{array}$ & $\begin{array}{l}0 \\
0 \\
.04 \\
.29 \\
.02\end{array}$ & $\begin{array}{l}.02 \\
.73 \\
.01 \\
.02 \\
.16\end{array}$ & $\begin{array}{l}0 \\
0 \\
0 \\
.05 \\
.12\end{array}$ & $\begin{array}{l}0 \\
0 \\
.08 \\
.04\end{array}$ & $\begin{array}{l}0 \\
0 \\
0_{0}^{.08} \\
0\end{array}$ \\
\hline $\begin{array}{r}6 \\
7 \\
8 \\
9 \\
10\end{array}$ & $\begin{array}{l}0 \\
0 \\
0 \\
0 \\
0\end{array}$ & $0_{0}^{.06}$ & $\begin{array}{l}0 \\
0 \\
0 \\
0 \\
0\end{array}$ & $\begin{array}{l}0 \\
0 \\
.05 \\
.02 \\
0.02\end{array}$ & $\begin{array}{l}0 \\
0 \\
0 \\
0 \\
0\end{array}$ & $\begin{array}{l}0 \\
0 \\
.51 \\
.02\end{array}$ & $\begin{array}{l}0 \\
0 \\
0 \\
0 \\
.54\end{array}$ & $\begin{array}{l}0 \\
0.01 \\
1.38 \\
.06\end{array}$ & $\begin{array}{l}--- \\
--- \\
0--\end{array}$ & $\begin{array}{l}0 \\
0^{0} .02 \\
.07\end{array}$ & $\begin{array}{l}--- \\
--- \\
.05 \\
.01\end{array}$ & $\begin{array}{l}0_{0}^{.04} \\
0 \\
0 \\
0\end{array}$ \\
\hline $\begin{array}{l}11 \\
12 \\
13 \\
14 \\
15\end{array}$ & $\begin{array}{l}0 \\
0 \\
0 \\
0 \\
.29\end{array}$ & $\begin{array}{l}0 \\
0 \\
0 \\
0 \\
0\end{array}$ & $\begin{array}{l}0 \\
0 \\
0 \\
0 \\
0\end{array}$ & $\begin{array}{l}0 \\
0 \\
0 \\
0 \\
0\end{array}$ & $\begin{array}{l}0 \\
0 \\
0 \\
.01 \\
.04\end{array}$ & $\begin{array}{r}.64 \\
.13 \\
1.56 \\
.39 \\
.17\end{array}$ & $\begin{array}{l}.47 \\
.16 \\
.05 \\
.05 \\
.12\end{array}$ & $\begin{array}{r}.03 \\
.04 \\
0.01 \\
.61\end{array}$ & $\begin{array}{l}0 \\
1.15 \\
.27 \\
.02 \\
.40\end{array}$ & $\begin{array}{l}.09 \\
.50 \\
.01 \\
.02 \\
.01\end{array}$ & $\begin{array}{l}0^{.02} \\
0 \\
0 \\
0\end{array}$ & $\begin{array}{l}.-- \\
.02 \\
.30\end{array}$ \\
\hline $\begin{array}{l}16 \\
17 \\
18 \\
19 \\
20\end{array}$ & $\begin{array}{l}0^{.61} \\
0 \\
0 \\
0\end{array}$ & $\begin{array}{l}0^{.01} \\
0 \\
0 \\
0\end{array}$ & $\begin{array}{l}0 \\
0 \\
0 \\
0 \\
0\end{array}$ & $\begin{array}{l}0^{.04} \\
0 \\
0 \\
.09\end{array}$ & $\begin{array}{l}0^{.11} \\
0 \\
0 \\
0\end{array}$ & $\begin{array}{l}.01 \\
0^{.01} \\
0 \\
.17\end{array}$ & $\begin{array}{l}0 \\
0 \\
.11 \\
.21 \\
.02\end{array}$ & $\begin{array}{l}0^{.03} \\
0 \\
1.44 \\
0\end{array}$ & $\begin{array}{r}.66 \\
.61 \\
.02 \\
.31 \\
0^{.31}\end{array}$ & $\begin{array}{r}.02 \\
.03 \\
3.09 \\
1.64 \\
.04\end{array}$ & $\begin{array}{r}.01 \\
1.76 \\
.50 \\
2.65 \\
.26\end{array}$ & $\begin{array}{l}0 \\
0 \\
0.25 \\
0.01\end{array}$ \\
\hline $\begin{array}{l}21 \\
22 \\
23 \\
24 \\
25\end{array}$ & $\begin{array}{l}0 \\
0 \\
0 \\
0 \\
0\end{array}$ & $\begin{array}{l}0 \\
0 \\
0 \\
0 \\
0\end{array}$ & $\begin{array}{l}0 \\
0 \\
0 \\
0 \\
0\end{array}$ & $\begin{array}{l}0 \\
0 \\
.04 \\
.04\end{array}$ & $\begin{array}{l}0 \\
0 \\
.07 \\
.01\end{array}$ & $0_{0}^{.01}$ & $\begin{array}{l}0 \\
0 \\
0.07 \\
0 \\
0\end{array}$ & $\begin{array}{l}0 \\
0 \\
0 \\
0 \\
.13 \\
.17\end{array}$ & $\begin{array}{l}.11 \\
1.16 \\
0 \\
0 \\
0\end{array}$ & $\begin{array}{l}0 \\
0 \\
0_{0}^{.03}\end{array}$ & $\begin{array}{l}0 \\
0 \\
.01 \\
3.67 \\
5.23\end{array}$ & $\begin{array}{l}0_{0}^{.03} \\
0 \\
0 \\
0\end{array}$ \\
\hline $\begin{array}{l}26 \\
27 \\
28 \\
29 \\
30 \\
31\end{array}$ & $\begin{array}{l}0 \\
0 \\
.25 \\
.30 \\
.52 \\
.03\end{array}$ & $\begin{array}{l}0 \\
0 \\
0 \\
0 \\
0\end{array}$ & $\begin{array}{l}0 \\
0 \\
0 \\
0 \\
0\end{array}$ & $\begin{array}{l}0 \\
0 \\
0 \\
0 \\
0 \\
0\end{array}$ & $\begin{array}{l}0 \\
0 \\
0\end{array}$ & $\begin{array}{l}0 \\
0 \\
0 \\
0 \\
0 \\
0\end{array}$ & $\begin{array}{l}.03 \\
.03 \\
.18 \\
.01 \\
.04\end{array}$ & $\begin{array}{l}0 \\
.03 \\
.54 \\
0 \\
0 \\
0\end{array}$ & $\begin{array}{r}.95 \\
.05 \\
.09 \\
.12 \\
0^{.12}\end{array}$ & $\begin{array}{r}.31 \\
.79 \\
.18 \\
0.02 \\
0^{.02}\end{array}$ & $\begin{array}{l}0^{.16} \\
0 \\
0 \\
0\end{array}$ & $\begin{array}{l}0.01 \\
0.02\end{array}$ \\
\hline $\begin{array}{l}\text { tal } \\
\text { asured }\end{array}$ & 2.67 & .25 & 0 & .29 & .31 & 3.80 & 2.41 & 4.83 & 6.86 & 7.04 & 14.45 & .76 \\
\hline
\end{tabular}


Table 7. Daily mean discharge at site DC5, Deer Creek near Postville, Iowa, water year 1990

[Discharge in cubic feet per second; ---, data not collected]

\begin{tabular}{|c|c|c|c|c|c|c|c|c|c|c|c|c|}
\hline Day & Oct. & Nov. & Dec. & Jan. & Feb. & Mar. & Apr. & May & June & July & Aug. & Sept. \\
\hline $\begin{array}{l}1 \\
2 \\
3 \\
4 \\
5\end{array}$ & $\begin{array}{r}0.24 \\
.20 \\
.20 \\
.21 \\
.39\end{array}$ & $\begin{array}{r}0.37 \\
.34 \\
.34 \\
.35 \\
.32\end{array}$ & $\begin{array}{r}0.27 \\
.23 \\
.23 \\
.23 \\
.26\end{array}$ & $\begin{array}{r}0.19 \\
.19 \\
.18 \\
.19 \\
-1-\end{array}$ & $\begin{array}{l}--- \\
--- \\
--- \\
---\end{array}$ & $\begin{array}{l}--- \\
=-- \\
--- \\
---\end{array}$ & $\begin{array}{l}--- \\
--- \\
--- \\
---\end{array}$ & $\begin{array}{l}--- \\
--19 \\
.19 \\
.21 \\
.23\end{array}$ & $\begin{array}{r}0.17 \\
.83 \\
.31 \\
.24 \\
.25\end{array}$ & $\begin{array}{r}0.17 \\
.17 \\
.17 \\
.15 \\
.14\end{array}$ & $\begin{array}{r}0.25 \\
.21 \\
.21 \\
1.6 \\
.34\end{array}$ & $\begin{array}{r}0.25 \\
.24 \\
.24 \\
.22 \\
.20\end{array}$ \\
\hline $\begin{array}{r}6 \\
7 \\
8 \\
9 \\
10\end{array}$ & $\begin{array}{l}.25 \\
.22 \\
.23 \\
.23 \\
.23\end{array}$ & $\begin{array}{l}.32 \\
.36 \\
.31 \\
.29 \\
.27\end{array}$ & $\begin{array}{l}.24 \\
.32 \\
.24 \\
.23 \\
.21\end{array}$ & $\begin{array}{l}--- \\
=-- \\
--- \\
---\end{array}$ & $\begin{array}{l}--- \\
-\because- \\
--- \\
---\end{array}$ & $\begin{array}{l}--- \\
-\because- \\
--- \\
---\end{array}$ & $\begin{array}{l}--- \\
-\because \\
--- \\
---\end{array}$ & $\begin{array}{l}.21 \\
.19 \\
.18 \\
.33 \\
.23\end{array}$ & $\begin{array}{l}.24 \\
.21 \\
.19 \\
.17 \\
.15\end{array}$ & $\begin{array}{l}.13 \\
.15 \\
.13 \\
.12 \\
.14\end{array}$ & $\begin{array}{l}.28 \\
.24 \\
.22 \\
.20 \\
.20\end{array}$ & $\begin{array}{l}.20 \\
.20 \\
.20 \\
.19 \\
.18\end{array}$ \\
\hline $\begin{array}{l}11 \\
12 \\
13 \\
14 \\
15\end{array}$ & $\begin{array}{l}.24 \\
.25 \\
.25 \\
.25 \\
.30\end{array}$ & $\begin{array}{l}.27 \\
.27 \\
.27 \\
.27 \\
.27\end{array}$ & $\begin{array}{l}.23 \\
.22 \\
.23 \\
.21 \\
.20\end{array}$ & $\begin{array}{l}--- \\
--- \\
--- \\
---\end{array}$ & $\begin{array}{l}--- \\
--- \\
--- \\
---\end{array}$ & $\begin{array}{l}--- \\
--- \\
--- \\
---\end{array}$ & $\begin{array}{l}--- \\
--- \\
--- \\
---\end{array}$ & $\begin{array}{l}.25 \\
.23 \\
.25 \\
.26 \\
.31\end{array}$ & $\begin{array}{l}.15 \\
. .55 \\
1.5 \\
.38 \\
1.2\end{array}$ & $\begin{array}{l}.16 \\
.28 \\
.19 \\
.17 \\
.16\end{array}$ & $\begin{array}{l}.20 \\
.18 \\
.18 \\
.17 \\
.15\end{array}$ & $\begin{array}{l}.18 \\
.17 \\
.17 \\
.17 \\
.16\end{array}$ \\
\hline $\begin{array}{l}16 \\
17 \\
18 \\
19 \\
20\end{array}$ & $\begin{array}{l}.65 \\
.31 \\
.31 \\
.32 \\
.32\end{array}$ & $\begin{array}{l}.26 \\
.27 \\
.28 \\
.29 \\
.30\end{array}$ & $\begin{array}{l}.19 \\
.19 \\
.19 \\
.26 \\
.20\end{array}$ & $\begin{array}{l}--- \\
--- \\
--- \\
--\end{array}$ & $\begin{array}{l}--- \\
--- \\
--- \\
---\end{array}$ & $\begin{array}{l}--- \\
=- \\
--- \\
---\end{array}$ & $\begin{array}{l}--- \\
--- \\
--- \\
---\end{array}$ & $\begin{array}{l}.28 \\
.30 \\
.30 \\
.29 \\
.30\end{array}$ & $\begin{array}{r}.70 \\
1.3 \\
.47 \\
.44 \\
.36\end{array}$ & $\begin{array}{l}.15 \\
.14 \\
.41 \\
.55 \\
.43\end{array}$ & $\begin{array}{l}.15 \\
.31 \\
.46 \\
.68 \\
.75\end{array}$ & $\begin{array}{r}.16 \\
.16 \\
.17 \\
.18 \\
.17\end{array}$ \\
\hline $\begin{array}{l}21 \\
22 \\
23 \\
24 \\
25\end{array}$ & $\begin{array}{l}.31 \\
.29 \\
.29 \\
.29 \\
.29\end{array}$ & $\begin{array}{l}.29 \\
.29 \\
.27 \\
.32 \\
.31\end{array}$ & $\begin{array}{l}.18 \\
.177 \\
.16 \\
.17 \\
.19\end{array}$ & $\begin{array}{l}-- \\
-- \\
--- \\
--\end{array}$ & $\begin{array}{l}--- \\
--- \\
--- \\
--\end{array}$ & $\begin{array}{l}--- \\
=- \\
--- \\
--\end{array}$ & $\begin{array}{l}--- \\
--- \\
--- \\
--\end{array}$ & $\begin{array}{l}.29 \\
.28 \\
.30 \\
.26 \\
.33\end{array}$ & $\begin{array}{r}.30 \\
2.4 \\
.60 \\
.43 \\
.33\end{array}$ & $\begin{array}{l}.27 \\
.22 \\
.20 \\
.20 \\
.18\end{array}$ & $\begin{array}{r}.49 \\
.38 \\
.30 \\
2.7 \\
1.1\end{array}$ & $\begin{array}{l}.16 \\
.16 \\
.16 \\
.16 \\
.14\end{array}$ \\
\hline $\begin{array}{l}26 \\
27 \\
28 \\
29 \\
30 \\
31\end{array}$ & $\begin{array}{l}.29 \\
.29 \\
.29 \\
.35 \\
. .59 \\
.45\end{array}$ & $\begin{array}{l}.30 \\
.28 \\
.27 \\
.27 \\
.25\end{array}$ & $\begin{array}{l}.17 \\
.17 \\
.18 \\
.18 \\
.19 \\
.20\end{array}$ & $\begin{array}{l}--- \\
--- \\
--- \\
--- \\
---\end{array}$ & \begin{tabular}{l}
--- \\
\hdashline-
\end{tabular} & $\begin{array}{l}--- \\
=- \\
z-- \\
--- \\
--\end{array}$ & $\begin{array}{l}--- \\
--- \\
--- \\
---\end{array}$ & $\begin{array}{l}.31 \\
.27 \\
.33 \\
.28 \\
.20 \\
.18\end{array}$ & $\begin{array}{l}.29 \\
.25 \\
.25 \\
.28 \\
.21\end{array}$ & $\begin{array}{l}.22 \\
.65 \\
.47 \\
.50 \\
.33 \\
.27\end{array}$ & $\begin{array}{c}1.5 \\
.80 \\
.51 \\
.42 \\
.34 \\
.29\end{array}$ & $\begin{array}{l}.14 \\
.13 \\
.13 \\
.13 \\
.13\end{array}$ \\
\hline
\end{tabular}


Table 8. Onsite determinations of selected water-quality constituents at surface-water monitoring sites in the Big Spring basin, Clayton County, Iowa, water year 1990

$\left[\mathrm{ft}^{3} / \mathrm{s}\right.$, cubic feet per second; $\mu \mathrm{S} / \mathrm{cm}$, microsiemens per centimeter at 25 degrees Celsius; ${ }^{\circ} \mathrm{C}$, degrees Celsius; $\mathrm{mg} / \mathrm{L}$, milligrams per liter; --, missing data]

\begin{tabular}{|c|c|c|c|c|c|c|}
\hline Date & $\begin{array}{c}\text { Time } \\
\text { (24-hour) }\end{array}$ & $\begin{array}{l}\text { Instan- } \\
\text { taneous } \\
\text { dis- } \\
\text { charge } \\
\left(\mathrm{ft}^{3} / \mathrm{s}\right)\end{array}$ & $\begin{array}{l}\text { Spe- } \\
\text { cific } \\
\text { con- } \\
\text { duct- } \\
\text { ance } \\
(\mu \mathrm{S} / \mathrm{cm})\end{array}$ & $\begin{array}{c}\mathrm{pH} \\
\text { (standard } \\
\text { units) }\end{array}$ & $\begin{array}{l}\text { Water } \\
\text { temper- } \\
\text { ature } \\
\left({ }^{\circ} \mathrm{C}\right)\end{array}$ & $\begin{array}{l}\text { Dissolve } \\
\text { oxygen } \\
\text { (mg/L) }\end{array}$ \\
\hline & \multicolumn{6}{|c|}{ Site DC5, Deer Creek (fig. 2) } \\
\hline $\begin{array}{l}10-04-89 \\
10-05-89 \\
11-08-89 \\
12-05-89 \\
01-04-90\end{array}$ & $\begin{array}{l}1145 \\
1300 \\
0800 \\
1000 \\
0900\end{array}$ & $\begin{array}{r}0.25 \\
.80 \\
.18 \\
.17 \\
.-\end{array}$ & $\begin{array}{l}554 \\
540 \\
550 \\
525 \\
553\end{array}$ & $\begin{array}{l}8.2 \\
7.4 \\
7.9 \\
8.0 \\
7.8\end{array}$ & $\begin{array}{l}7.0 \\
9.0 \\
7.5 \\
1.0 \\
1.0\end{array}$ & $\begin{array}{l}14.3 \\
-- \\
12.3 \\
14.5 \\
11.2\end{array}$ \\
\hline $\begin{array}{l}02-07-90 \\
02-08-90 \\
02-08-90 \\
03-06-90 \\
03-08-90\end{array}$ & $\begin{array}{l}1015 \\
1200 \\
1425 \\
0845 \\
2000\end{array}$ & $\begin{array}{l}.26 \\
.59 \\
-- \\
3.0\end{array}$ & $\begin{array}{l}528 \\
400 \\
248 \\
578 \\
315\end{array}$ & $\begin{array}{l}7.7 \\
7.7 \\
7.6 \\
7.9 \\
7.6\end{array}$ & $\begin{array}{l}0 \\
0.5 \\
.5 \\
0.0 \\
1.0\end{array}$ & $\begin{array}{l}11.4 \\
-. \\
-- \\
13.3 \\
--\end{array}$ \\
\hline $\begin{array}{l}03-09-90 \\
03-16-90 \\
04-03-90 \\
05-02-90 \\
05-09-90\end{array}$ & $\begin{array}{l}1325 \\
1115 \\
0840 \\
0900 \\
0415\end{array}$ & $\begin{array}{l}.82 \\
-- \\
-. \\
.18 \\
.31\end{array}$ & $\begin{array}{l}435 \\
648 \\
567 \\
573 \\
572\end{array}$ & $\begin{array}{l}7.6 \\
7.8 \\
8.0 \\
8.2 \\
7.6\end{array}$ & $\begin{array}{r}2.0 \\
6.0 \\
2.0 \\
7.0 \\
13.0\end{array}$ & $\begin{array}{l}13.2 \\
-- \\
-- \\
13.8 \\
--\end{array}$ \\
\hline $\begin{array}{l}05-09-90 \\
05-09-90 \\
06-05-90 \\
06-13-90 \\
06-13-90\end{array}$ & $\begin{array}{l}0830 \\
1200 \\
1045 \\
1045 \\
1115\end{array}$ & $\begin{array}{l}.68 \\
.47 \\
.21 \\
2.8 \\
7.1\end{array}$ & $\begin{array}{l}490 \\
460 \\
585 \\
300 \\
231\end{array}$ & $\begin{array}{l}7.8 \\
7.7 \\
7.7 \\
7.2 \\
7.2\end{array}$ & $\begin{array}{l}12.0 \\
12.0 \\
11.0 \\
17.0 \\
17.0\end{array}$ & $\begin{array}{l}7.9 \\
-- \\
-- \\
-- \\
--\end{array}$ \\
\hline $\begin{array}{l}06-22-90 \\
06-22-90 \\
06-23-90 \\
07-02-90 \\
08-02-90\end{array}$ & $\begin{array}{l}1130 \\
1445 \\
0900 \\
0900 \\
0850\end{array}$ & $\begin{array}{l}4.1 \\
2.0 \\
.62 \\
.17 \\
.21\end{array}$ & $\begin{array}{l}425 \\
500 \\
615 \\
600 \\
592\end{array}$ & $\begin{array}{l}7.1 \\
7.3 \\
7.4 \\
7.4 \\
-.\end{array}$ & $\begin{array}{l}15.0 \\
16.0 \\
15.0 \\
17.0 \\
13.5\end{array}$ & $\begin{array}{l}\ldots \\
\ldots \\
\cdots \\
9.5 \\
10.1\end{array}$ \\
\hline $\begin{array}{l}08-20-90 \\
08-26-90 \\
09-06-90\end{array}$ & $\begin{array}{l}1410 \\
1340 \\
0750\end{array}$ & $\begin{array}{l}.43 \\
.88 \\
.21\end{array}$ & $\begin{array}{l}615 \\
680 \\
615\end{array}$ & $\begin{array}{l}-- \\
7.5 \\
7.8\end{array}$ & $\begin{array}{l}18.0 \\
22.0 \\
17.0\end{array}$ & $\begin{array}{l}-- \\
-- \\
6.7\end{array}$ \\
\hline
\end{tabular}


Table 8. Onsite determinations of selected water-quality constituents at surface-water monitoring sites in the Big Spring basin, Clayton County, Iowa, water year 1990--Continued

\begin{tabular}{|c|c|c|c|c|c|c|}
\hline & & Instan- & $\begin{array}{l}\text { Spe- } \\
\text { cific }\end{array}$ & & & \\
\hline Date & $\begin{array}{c}\text { Time } \\
\text { (24-hour) }\end{array}$ & $\begin{array}{l}\text { taneous } \\
\text { dis- } \\
\text { charge } \\
\left(\mathrm{ft} t^{3} / \mathrm{s}\right)\end{array}$ & $\begin{array}{l}\text { con- } \\
\text { duct- } \\
\text { ance } \\
(\mu S / c m)\end{array}$ & $\begin{array}{c}\mathrm{pH} \\
\text { (standard } \\
\text { units) }\end{array}$ & $\begin{array}{l}\text { Water } \\
\text { temper- } \\
\text { ature } \\
\left({ }^{\circ} \mathrm{C}\right)\end{array}$ & $\begin{array}{l}\text { Dissolved } \\
\text { oxygen } \\
(\mathrm{mg} / \mathrm{L})\end{array}$ \\
\hline
\end{tabular}

Site BOOGD, Unnamed Creek near Luana (fig. 2)

\begin{tabular}{|c|c|c|c|c|c|c|}
\hline $\begin{array}{l}02-06-90 \\
03-08-90 \\
03-08-90 \\
06-13-90 \\
06-22-90\end{array}$ & $\begin{array}{l}1200 \\
1300 \\
2250 \\
1305 \\
1150\end{array}$ & $\begin{array}{c}-- \\
24 \\
1.3 \\
5.0 \\
4.7\end{array}$ & $\begin{array}{l}345 \\
280 \\
360 \\
220 \\
340\end{array}$ & $\begin{array}{l}7.8 \\
7.6 \\
7.6 \\
7.0 \\
7.1\end{array}$ & $\begin{array}{r}0 \\
.5 \\
1.0 \\
19.0 \\
15.0\end{array}$ & $\begin{array}{l}13.3 \\
-- \\
-- \\
-- \\
--\end{array}$ \\
\hline $\begin{array}{l}06-22-90 \\
06-23-90 \\
07-20-90 \\
08-01-90 \\
08-07-90\end{array}$ & $\begin{array}{l}1600 \\
1000 \\
1400 \\
1625 \\
0815\end{array}$ & $\begin{array}{r}1.3 \\
.09 \\
.09 \\
.05 \\
.04\end{array}$ & $\begin{array}{r}540 \\
880 \\
1,000 \\
1,100 \\
-\end{array}$ & $\begin{array}{r}7.1 \\
7.8 \\
-- \\
7.9 \\
-.\end{array}$ & $\begin{array}{l}18.0 \\
17.0 \\
30.0 \\
28.5 \\
15.0\end{array}$ & $\begin{array}{l}\ldots \\
6.0 \\
6.8 \\
\ldots\end{array}$ \\
\hline $\begin{array}{l}08-20-90 \\
08-26-90 \\
09-05-90\end{array}$ & $\begin{array}{l}1215 \\
1445 \\
1540\end{array}$ & $13^{.24}$ & $\begin{array}{l}765 \\
790 \\
870\end{array}$ & $\begin{array}{l}8.4 \\
7.6 \\
7.6\end{array}$ & $\begin{array}{l}20.0 \\
23.0 \\
27.5\end{array}$ & $\begin{array}{l}\cdots \\
4.0\end{array}$ \\
\hline
\end{tabular}

Site RC2, Roberts Creek above Saint Olaf (fig. 2)

\begin{tabular}{|c|c|c|c|c|c|c|}
\hline $\begin{array}{l}01-22-90 \\
01-31-90 \\
02-06-90 \\
02-07-90 \\
02-07-90\end{array}$ & $\begin{array}{l}1230 \\
1415 \\
0930 \\
1320 \\
1430\end{array}$ & $\begin{array}{r}7.5 \\
3.1 \\
191 \\
38 \\
37\end{array}$ & $\begin{array}{r}500 \\
672 \\
350 \\
-- \\
297\end{array}$ & $\begin{array}{r}7.4 \\
7.7 \\
7.7 \\
7.7\end{array}$ & $\begin{array}{l}0.5 \\
2.0 \\
0 \\
0\end{array}$ & $\begin{array}{l}\cdots \\
- \\
\cdots \\
\cdots \\
12.2\end{array}$ \\
\hline $\begin{array}{l}02-08-90 \\
03-06-90 \\
03-08-90 \\
03-08-90 \\
03-08-90\end{array}$ & $\begin{array}{l}1500 \\
1300 \\
1630 \\
1915 \\
1950\end{array}$ & $\begin{array}{r}191 \\
3.5 \\
483 \\
1,210 \\
1,180\end{array}$ & $\begin{array}{l}365 \\
450 \\
255 \\
315 \\
290\end{array}$ & $\begin{array}{l}7.7 \\
7.7 \\
7.7 \\
7.6 \\
7.7\end{array}$ & $\begin{array}{l}0^{.5} \\
5.0 \\
1.0 \\
1.5\end{array}$ & $\begin{array}{l}-- \\
12.0 \\
-- \\
-- \\
12.0\end{array}$ \\
\hline $\begin{array}{l}03-09-90 \\
03-10-90 \\
03-12-90 \\
03-16-90 \\
04-03-90\end{array}$ & $\begin{array}{l}0845 \\
0720 \\
1000 \\
1430 \\
1345\end{array}$ & $\begin{array}{c}153 \\
34 \\
71 \\
22 \\
2.6\end{array}$ & $\begin{array}{l}320 \\
352 \\
446 \\
690 \\
640\end{array}$ & $\begin{array}{l}7.6 \\
7.6 \\
7.7 \\
8.2 \\
9.1\end{array}$ & $\begin{array}{l}0 \\
1.5 \\
2.0 \\
7.0 \\
8.5\end{array}$ & $\begin{array}{l}13.2 \\
12 \\
\ldots \\
\ldots \\
\ldots\end{array}$ \\
\hline
\end{tabular}


Table 8. Onsite determinations of selected water-quality constituents at surface-water monitoring sites in the Big Spring basin, Clayton County, Iowa, water year 1990-Continued

\begin{tabular}{|c|c|c|c|c|c|c|}
\hline & & Instan- & $\begin{array}{l}\text { Spe- } \\
\text { cific }\end{array}$ & & & \\
\hline Date & $\begin{array}{c}\text { Time } \\
(24-\text { hour })\end{array}$ & $\begin{array}{l}\text { taneous } \\
\text { dis- } \\
\text { charge } \\
\left(f t^{3} / \mathrm{s}\right)\end{array}$ & $\begin{array}{l}\text { con- } \\
\text { duct- } \\
\text { ance } \\
(\mu S / \mathrm{cm})\end{array}$ & $\begin{array}{c}\mathrm{pH} \\
\text { (standard } \\
\text { units) }\end{array}$ & $\begin{array}{l}\text { Water } \\
\text { temper- } \\
\text { ature } \\
\left({ }^{\circ} \mathrm{C}\right)\end{array}$ & $\begin{array}{l}\text { Dissolved } \\
\text { oxygen } \\
\text { (mg/L) }\end{array}$ \\
\hline
\end{tabular}

Site RC2, Roberts Creek above Saint 0laf--continued

\begin{tabular}{|c|c|c|c|c|c|c|}
\hline $\begin{array}{l}04-14-90 \\
05-01-90 \\
05-20-90 \\
06-06-90 \\
06-17-90\end{array}$ & $\begin{array}{l}0110 \\
1030 \\
1200 \\
1320 \\
1410\end{array}$ & $\begin{array}{l}2.9 \\
.84 \\
31 \\
6.9 \\
28\end{array}$ & $\begin{array}{l}523 \\
621 \\
-- \\
630 \\
572\end{array}$ & $\begin{array}{l}8.6 \\
8.2 \\
8.2 \\
7.8 \\
7.8\end{array}$ & $\begin{array}{r}5.5 \\
12.5 \\
12.0 \\
16.0 \\
22.0\end{array}$ & $\begin{array}{c}-- \\
9.6 \\
-- \\
9.2 \\
--\end{array}$ \\
\hline $\begin{array}{l}06-22-90 \\
06-22-90 \\
06-23-90 \\
07-03-90 \\
07-16-90\end{array}$ & $\begin{array}{l}1300 \\
1730 \\
0630 \\
0730 \\
1330\end{array}$ & $\begin{array}{l}41 \\
46 \\
73 \\
8.2 \\
2.6\end{array}$ & $\begin{array}{l}666 \\
534 \\
608 \\
742 \\
606\end{array}$ & $\begin{array}{l}7.6 \\
7.6 \\
7.3 \\
8.1 \\
8.0\end{array}$ & $\begin{array}{l}21.0 \\
19.5 \\
17.0 \\
23.0 \\
28.0\end{array}$ & $\begin{array}{l}\ldots \\
\ldots \\
\cdots \\
6.0 \\
\ldots\end{array}$ \\
\hline $\begin{array}{l}07-20-90 \\
08-02-90 \\
08-08-90 \\
08-19-90 \\
08-20-90\end{array}$ & $\begin{array}{l}1000 \\
1330 \\
1910 \\
0255 \\
0115\end{array}$ & $\begin{array}{l}37 \\
6.1 \\
5.3 \\
15 \\
19\end{array}$ & $\begin{array}{l}516 \\
690 \\
603 \\
553 \\
577\end{array}$ & $\begin{array}{l}7.7 \\
8.4 \\
8.6 \\
8.1 \\
8.1\end{array}$ & $\begin{array}{l}20.5 \\
24.0 \\
25.0 \\
24.0 \\
22.5\end{array}$ & $\begin{array}{l}7.0 \\
12.1 \\
-- \\
-- \\
--\end{array}$ \\
\hline $\begin{array}{l}08-20-90 \\
08-20-90 \\
08-24-90 \\
08-25-90 \\
08-25-90\end{array}$ & $\begin{array}{l}0515 \\
1115 \\
2050 \\
0755 \\
1205\end{array}$ & $\begin{array}{r}27 \\
23 \\
19 \\
573 \\
437\end{array}$ & $\begin{array}{l}631 \\
596 \\
667 \\
588 \\
462\end{array}$ & $\begin{array}{l}8.1 \\
8.0 \\
8.1 \\
5.4 \\
7.1\end{array}$ & $\begin{array}{l}21.5 \\
21.0 \\
23.5 \\
25.5 \\
19.5\end{array}$ & $\begin{array}{l}-- \\
\ldots \\
- \\
-- \\
--\end{array}$ \\
\hline $\begin{array}{l}08-25-90 \\
08-25-90 \\
08-26-90 \\
08-26-90 \\
08-26-90\end{array}$ & $\begin{array}{l}1455 \\
2135 \\
0135 \\
1015 \\
1610\end{array}$ & $\begin{array}{r}751 \\
262 \\
176 \\
113 \\
97\end{array}$ & $\begin{array}{l}480 \\
508 \\
545 \\
588 \\
629\end{array}$ & $\begin{array}{l}7.2 \\
7.3 \\
7.4 \\
7.7 \\
7.8\end{array}$ & $\begin{array}{l}19.5 \\
20.0 \\
20.0 \\
20.0 \\
22.0\end{array}$ & $\begin{array}{l}- \\
- \\
-- \\
-- \\
--\end{array}$ \\
\hline $\begin{array}{l}08-27-90 \\
08-27-90 \\
08-28-90 \\
08-30-90 \\
09-06-90\end{array}$ & $\begin{array}{l}0535 \\
1340 \\
1320 \\
1050 \\
1210\end{array}$ & $\begin{array}{l}72 \\
62 \\
42 \\
27 \\
\quad .42\end{array}$ & $\begin{array}{l}693 \\
775 \\
787 \\
796 \\
736\end{array}$ & $\begin{array}{l}7.7 \\
8.0 \\
8.0 \\
8.0 \\
8.2\end{array}$ & $\begin{array}{l}21.0 \\
23.0 \\
25.0 \\
20.0 \\
26.0\end{array}$ & $\begin{array}{l}\cdots \\
\cdots \\
\cdots \\
11.8\end{array}$ \\
\hline
\end{tabular}


Table 9. Concentrations of major ions at selected surface-water monitoring sites in the Big Spring basin, Clayton County, Iowa, water year 1990

[Dissolved constituents are in milligrams per liter; --, data not collected; <, less than]

\begin{tabular}{|c|c|c|c|c|c|c|c|c|c|c|}
\hline Date & $\begin{array}{c}\text { Time } \\
\text { (24-hour) }\end{array}$ & Calcium & $\begin{array}{l}\text { Mag- } \\
\text { ne- } \\
\text { sium }\end{array}$ & $\begin{array}{l}\text { So- } \\
\text { dium }\end{array}$ & $\begin{array}{l}\text { Pot- } \\
\text { tas- } \\
\text { sium }\end{array}$ & $\begin{array}{l}\text { Bi- } \\
\text { carbo- } \\
\text { nate }\end{array}$ & $\begin{array}{l}\text { Car- } \\
\text { bo- } \\
\text { nate }\end{array}$ & $\begin{array}{l}\text { Sul- } \\
\text { fate }\end{array}$ & $\begin{array}{l}\text { Chlo- } \\
\text { ride }\end{array}$ & Silica \\
\hline
\end{tabular}

Site DC5, Deer Creek (fig. 2)

$\begin{array}{rrrrrrlllll}10-04-89 & 1145 & 68 & 23 & 4.0 & 1.2 & - & -- & 26 & 11 & 24 \\ 11-08-89 & 0800 & 85 & 28 & 4.7 & 1.6 & 360 & 0 & 31 & 12 & 18 \\ 12-05-89 & 1000 & 80 & 28 & 4.5 & 0.7 & 330 & 0 & 26 & 9.5 & -- \\ 08-02-90 & 0850 & 110 & 30 & 5.3 & 1.0 & -- & -- & 34 & 11 & 24 \\ 09-06-90 & 0750 & 83 & 30 & 5.7 & 2.1 & 380 & 0 & 3.0 & 11 & 25\end{array}$

Site RC2, Roberts Creek above Saint Olaf (fig. 2)

$\begin{array}{rrrrrrrrrrr}03-12-90 & 1000 & 33 & 12 & 4.6 & 27 & -- & \ldots & 23 & 34 & 12 \\ 04-03-90 & 1345 & 73 & 31 & 16 & 6.7 & -- & -- & 46 & 35 & 4.1 \\ 06-06-90 & 1320 & 66 & 27 & 11 & 12 & 270 & 0 & 41 & 28 & -- \\ 07-20-90 & 1000 & 54 & 25 & 11 & 13 & -- & -- & -- & 25 & 7.4 \\ 08-02-90 & 1330 & 110 & 39 & 10 & 9.4 & -- & -- & 46 & 31 & 6.6 \\ 09-06-90 & 1210 & 80 & 40 & 11 & 4.8 & 260 & 50 & 44 & 28 & 8.5\end{array}$


Table 10. Selected nitrogen, phosphorus, and carbon species at surface-water monitoring sites in the Big Spring basin, Clayton County, Iowa, water year 1990

[Total constituents in milligrams per liter; N, nitrogen; P, phosphorus; C, carbon; --, data not collected; $<$, less than detection level indicated]

\begin{tabular}{|c|c|c|c|c|c|c|}
\hline Date & $\begin{array}{c}\text { Time } \\
\text { (24-hour) }\end{array}$ & $\begin{array}{l}\text { Nitrite } \\
\text { plus } \\
\text { nitrate, } \\
\text { total } \\
\text { (as } N)\end{array}$ & $\begin{array}{l}\text { Ammonia, } \\
\text { total } \\
\text { (as } N \text { ) }\end{array}$ & $\begin{array}{l}\text { Organic } \\
\text { nitrogen, } \\
\text { total } \\
\text { (as N) }\end{array}$ & $\begin{array}{l}\text { Ortho- } \\
\text { phos- } \\
\text { phorus, } \\
\text { total } \\
\text { (as P) }\end{array}$ & $\begin{array}{l}\text { Organic } \\
\text { carbon, } \\
\text { total } \\
\text { (as C) }\end{array}$ \\
\hline
\end{tabular}

Site DC5, Deer Creek (fig. 2)

\begin{tabular}{|c|c|c|c|c|c|c|}
\hline $\begin{array}{l}12-05-89 \\
01-04-90 \\
02-07-90 \\
02-08-90 \\
02-08-90\end{array}$ & $\begin{array}{l}1000 \\
0900 \\
1015 \\
1200 \\
1425\end{array}$ & $\begin{array}{r}3.3 \\
3.4 \\
2.7 \\
1.8 \\
.9\end{array}$ & $\begin{array}{c}<0.10 \\
.10 \\
2.8 \\
3.4 \\
3.1\end{array}$ & $\begin{array}{l}0.3 \\
.2 \\
3.4 \\
6.1 \\
8.6\end{array}$ & $\begin{array}{c}<0.10 \\
<\quad .10 \\
.40 \\
1.1 \\
2.0\end{array}$ & $\begin{array}{l}1.4 \\
2.0 \\
19 \\
28 \\
25\end{array}$ \\
\hline $\begin{array}{l}03-06-90 \\
03-08-90 \\
03-09-90 \\
03-16-90 \\
04-03-90\end{array}$ & $\begin{array}{l}0845 \\
2000 \\
1325 \\
1115 \\
0840\end{array}$ & $\begin{array}{l}3.3 \\
1.6 \\
2.8 \\
8.5 \\
3.1\end{array}$ & $\begin{array}{c}<.10 \\
3.8 \\
3.0 \\
.40 \\
<.10\end{array}$ & $\begin{array}{l}.4 \\
7.4 \\
5.4 \\
1.2 \\
.30\end{array}$ & $\begin{aligned}<.10 \\
1.8 \\
1.0 \\
.10 \\
<.10\end{aligned}$ & $\begin{array}{l}2.4 \\
43 \\
43 \\
8.0 \\
3.1\end{array}$ \\
\hline $\begin{array}{l}05-02-90 \\
05-09-90 \\
05-09-90 \\
05-09-90 \\
06-05-90\end{array}$ & $\begin{array}{l}0900 \\
0415 \\
0830 \\
1200 \\
1045\end{array}$ & $\begin{array}{r}2.1 \\
1.4 \\
.90 \\
.70 \\
3.5\end{array}$ & $\begin{array}{l}<.10 \\
<.10 \\
<.10 \\
<.10 \\
<.10\end{array}$ & $\begin{array}{r}.20 \\
.80 \\
1.5 \\
1.3 \\
.60\end{array}$ & $\begin{array}{r}<.10 \\
<.10 \\
.20 \\
<.10 \\
.20\end{array}$ & $\begin{array}{c}5.3 \\
5.3 \\
12 \\
12 \\
4.0\end{array}$ \\
\hline $\begin{array}{l}06-13-90 \\
06-13-90 \\
06-22-90 \\
06-22-90 \\
06-23-90\end{array}$ & $\begin{array}{l}1045 \\
1115 \\
1130 \\
1445 \\
0900\end{array}$ & $\begin{array}{l}1.5 \\
11 \\
3.5 \\
6.6 \\
9.0\end{array}$ & $\begin{array}{r}.30 \\
.40 \\
.20 \\
.20 \\
<.10\end{array}$ & $\begin{array}{l}-. \\
9.9 \\
3.0 \\
2.7 \\
1.3\end{array}$ & $\begin{aligned} & .80 \\
& 1.5 \\
& .60 \\
& .60 \\
&<.10\end{aligned}$ & $\begin{array}{l}41 \\
74 \\
18 \\
16 \\
10\end{array}$ \\
\hline $\begin{array}{l}07-02-90 \\
08-02-90 \\
08-20-90 \\
08-26-90 \\
09-06-90\end{array}$ & $\begin{array}{l}1000 \\
0850 \\
1410 \\
1340 \\
0750\end{array}$ & $\begin{array}{r}3.3 \\
2.8 \\
3.7 \\
17 \\
2.7\end{array}$ & $\begin{array}{r}<.10 \\
<.10 \\
.10 \\
.10 \\
<.10\end{array}$ & $\begin{array}{r}.30 \\
.90 \\
1.7 \\
2.5 \\
.50\end{array}$ & $\begin{array}{l}<.10 \\
<.10 \\
.- \\
.10 \\
.10\end{array}$ & $\begin{array}{c}3.5 \\
3.7 \\
-- \\
17 \\
4.7\end{array}$ \\
\hline
\end{tabular}


Table 10. Selected nitrogen, phosphorus, and carbon species at surface-water monitoring sites in the Big Spring basin, Clayton County, Iowa, water year 1990--Continued

\begin{tabular}{|c|c|c|c|c|c|c|}
\hline Date & $\begin{array}{c}\text { Time } \\
(24-\text { hour })\end{array}$ & $\begin{array}{l}\text { Nitrite } \\
\text { plus } \\
\text { nitrate, } \\
\text { total } \\
\text { (as N) }\end{array}$ & $\begin{array}{l}\text { Ammonia, } \\
\text { total } \\
\text { (as } N \text { ) }\end{array}$ & $\begin{array}{l}\text { Organic } \\
\text { nitrogen, } \\
\text { total } \\
\text { (as } N \text { ) }\end{array}$ & $\begin{array}{l}\text { Ortho- } \\
\text { phos- } \\
\text { phorus, } \\
\text { total } \\
\text { (as P) }\end{array}$ & $\begin{array}{l}\text { Organic } \\
\text { carbon, } \\
\text { total } \\
\text { (as C) }\end{array}$ \\
\hline
\end{tabular}

Site BOOGD, Unnamed Creek near Luana (fig. 2)

$\begin{array}{lcccccc}02-06-90 & 1200 & 2.5 & 3.0 & 7.1 & 1.6 & 29 \\ 02-08-90 & 1300 & 1.5 & 2.8 & 8.7 & 1.7 & 33 \\ 02-22-90 & 1120 & 2.0 & 2.3 & 4.4 & .9 & 35 \\ 03-08-90 & 2250 & 5.3 & 2.6 & 7.5 & 1.4 & 46 \\ 06-13-90 & 1305 & 9.4 & .20 & 7.9 & 1.2 & 48 \\ 06-22-90 & 1150 & 12 & .10 & 3.6 & .50 & 22 \\ 06-22-90 & 1600 & 7.0 & <. .10 & 1.8 & .40 & 15 \\ 06-23-90 & 1000 & 9.0 & <.10 & .9 & .20 & 4.0 \\ 07-02-90 & 1415 & 6.0 & 1.7 & 2.9 & .20 & 40 \\ 07-20-90 & 1400 & 5.0 & 2.9 & 14 & -- & 21 \\ 08-01-90 & 1625 & 14 & 5.3 & 7.1 & .20 & 9.8 \\ 08-07-90 & 0815 & 1.0 & .10 & .80 & -. & -- \\ 08-20-90 & 1215 & 6.0 & .10 & 1.0 & -. & -. \\ 08-26-90 & 1445 & 30 & .30 & 3.8 & .10 & 6.0 \\ 09-05-90 & 1540 & 9.0 & 1.8 & 11 & .10 & 9.8\end{array}$

Site RC2, Roberts Creek above Saint Olaf (fig. 2)

$\begin{array}{lllllll}01-22-90 & 1230 & 1.9 & 3.3 & -- & 1.6 & 40 \\ 01-31-90 & 1415 & .60 & 2.3 & 1.9 & -- & -- \\ 02-06-90 & 0930 & 2.0 & 3.7 & 8.6 & 2.10 & 22 \\ 02-07-90 & 1430 & 2.1 & 3.2 & 6.2 & 1.5 & 23 \\ 02-08-90 & 1500 & 2.1 & 4.0 & 7.1 & 1.9 & 27 \\ & & & & & & \\ 03-06-90 & 1300 & 1.3 & 2.5 & 2.6 & .60 & 17 \\ 03-08-90 & 1630 & 2.0 & 2.4 & 7.3 & 1.2 & 38 \\ 03-08-90 & 1915 & 2.2 & 3.2 & 13 & 1.2 & 93 \\ 03-08-90 & 1950 & 2.3 & 3.3 & 16 & .90 & 86 \\ 03-09-90 & 0845 & 3.0 & 3.4 & 10 & 1.9 & 47\end{array}$


Table 10. Selected nitrogen, phosphorus, and carbon species at surface-water monitoring sites in the Big Spring basin, Clayton County, Iowa, water year 1990--Continued

\begin{tabular}{|c|c|c|c|c|c|c|}
\hline Date & $\begin{array}{c}\text { Time } \\
\text { (24-hour) }\end{array}$ & $\begin{array}{l}\text { Nitrite } \\
\text { plus } \\
\text { nitrate, } \\
\text { total } \\
(\text { as } N)\end{array}$ & $\begin{array}{l}\text { Ammonia, } \\
\text { total } \\
\text { (as } N \text { ) }\end{array}$ & $\begin{array}{l}\text { Organic } \\
\text { nitrogen, } \\
\text { total } \\
\text { (as } N \text { ) }\end{array}$ & $\begin{array}{l}\text { Ortho- } \\
\text { phos- } \\
\text { phorus, } \\
\text { total } \\
\text { (as P) }\end{array}$ & $\begin{array}{l}\text { Organic } \\
\text { carbon, } \\
\text { total } \\
\text { (as C) }\end{array}$ \\
\hline
\end{tabular}

Site RC2, Roberts Creek above Saint Olaf--Continued

\begin{tabular}{|c|c|c|c|c|c|c|}
\hline $\begin{array}{l}03-10-90 \\
03-12-90 \\
03-16-90 \\
04-03-90 \\
04-14-90\end{array}$ & $\begin{array}{l}0720 \\
1000 \\
1430 \\
1345 \\
0110\end{array}$ & $\begin{array}{c}3.1 \\
6.4 \\
10 \\
2.7 \\
2.1\end{array}$ & $\begin{array}{r}3.6 \\
2.8 \\
1.8 \\
<\quad .10 \\
<.10\end{array}$ & $\begin{array}{l}8.0 \\
8.8 \\
2.9 \\
.50 \\
.80\end{array}$ & $\begin{array}{l}1.8 \\
1.0 \\
1.0 \\
.20 \\
-.\end{array}$ & $\begin{array}{c}48 \\
31 \\
15 \\
4.0 \\
\ldots\end{array}$ \\
\hline $\begin{array}{l}05-01-90 \\
05-20-90 \\
06-06-90 \\
06-17-90 \\
06-22-90\end{array}$ & $\begin{array}{l}1030 \\
1200 \\
1320 \\
1410 \\
1300\end{array}$ & $\begin{array}{c}<.10 \\
1.9 \\
7.0 \\
6.8 \\
12\end{array}$ & $\begin{array}{r}<.10 \\
.50 \\
.60 \\
.80 \\
<.10\end{array}$ & $\begin{array}{l}1.1 \\
1.7 \\
1.7 \\
2.4 \\
1.1\end{array}$ & $\begin{array}{l}.20 \\
-. \\
.60 \\
.90 \\
.30\end{array}$ & $\begin{array}{c}4.7 \\
-- \\
8.6 \\
12 \\
6.8\end{array}$ \\
\hline $\begin{array}{l}06-22-90 \\
06-23-90 \\
07-03-90 \\
07-16-90 \\
07-20-90\end{array}$ & $\begin{array}{l}1730 \\
0630 \\
0730 \\
1330 \\
1000\end{array}$ & $\begin{array}{c}9.1 \\
13 \\
9.5 \\
2.0 \\
2.8\end{array}$ & $\begin{array}{l}<.10 \\
<.4 \\
<.10 \\
<.10 \\
.4\end{array}$ & $\begin{array}{r}1.6 \\
2.0 \\
.8 \\
.8 \\
2.0\end{array}$ & $\begin{array}{r}.30 \\
.80 \\
.30 \\
-- \\
.70\end{array}$ & $\begin{array}{c}12 \\
16 \\
4.9 \\
-- \\
12\end{array}$ \\
\hline $\begin{array}{l}08-02-90 \\
08-08-90 \\
08-20-90 \\
08-20-90 \\
08-24-90\end{array}$ & $\begin{array}{l}1330 \\
1910 \\
0115 \\
1115 \\
2050\end{array}$ & $\begin{array}{l}4.3 \\
4.4 \\
1.3 \\
1.9 \\
4.8\end{array}$ & $\begin{array}{l}<.10 \\
-. \\
<.10 \\
.30 \\
<.10\end{array}$ & $\begin{array}{l}1.1 \\
1.7 \\
1.1 \\
1.9 \\
1.7\end{array}$ & $\begin{array}{l}.40 \\
-- \\
-. \\
.60\end{array}$ & $\begin{array}{l}6.8 \\
-- \\
-- \\
-- \\
8.3\end{array}$ \\
\hline $\begin{array}{l}08-25-90 \\
08-25-90 \\
08-25-90 \\
08-25-90 \\
08-26-90\end{array}$ & $\begin{array}{l}0755 \\
1205 \\
1455 \\
2135 \\
0135\end{array}$ & $\begin{array}{l}2.4 \\
2.6 \\
4.8 \\
7.7 \\
3.0\end{array}$ & $\begin{array}{l}.70 \\
1.2 \\
2.6 \\
1.0 \\
.70\end{array}$ & $\begin{array}{l}12 \\
15 \\
20 \\
7.1 \\
2.7\end{array}$ & $\begin{array}{l}.30 \\
.50 \\
.20 \\
.30 \\
.40\end{array}$ & $\begin{array}{l}6.7 \\
7.9 \\
9.4 \\
9.1 \\
8.6\end{array}$ \\
\hline $\begin{array}{l}08-26-90 \\
08-26-90 \\
08-27-90 \\
08-27-90 \\
08-28-90\end{array}$ & $\begin{array}{l}1015 \\
1610 \\
0535 \\
1340 \\
1320\end{array}$ & $\begin{array}{r}18 \\
2.0 \\
4.0 \\
3.3 \\
3.0\end{array}$ & $\begin{array}{r}.60 \\
.40 \\
.30 \\
.20 \\
<.10\end{array}$ & $\begin{array}{l}1.8 \\
1.8 \\
1.4 \\
1.2 \\
1.2\end{array}$ & $\begin{array}{l}.40 \\
.40 \\
.30 \\
.30 \\
.40\end{array}$ & $\begin{array}{r}8.2 \\
7.9 \\
7.5 \\
6.3 \\
--\end{array}$ \\
\hline $\begin{array}{l}09-06-90 \\
09-13-90\end{array}$ & $\begin{array}{l}1210 \\
0030\end{array}$ & $\begin{array}{l}9.8 \\
5.5\end{array}$ & $\begin{array}{l}<.10 \\
<.10\end{array}$ & $\begin{array}{l}.80 \\
.90\end{array}$ & .20 & 3.9 \\
\hline
\end{tabular}


Table 11. Selected pesticides at surface-water monitoring sites in the Big Spring basin, Clayton County, Iowa, water year 1990

[Total-recoverable constituents in micrograms per liter; $<$, less than detection level indicated]

\begin{tabular}{|c|c|c|c|c|c|c|c|c|}
\hline Date & $\begin{array}{c}\text { Time } \\
\text { (24-hour) }\end{array}$ & $\begin{array}{l}\text { Ala- } \\
\text { chlor }\end{array}$ & $\begin{array}{l}\text { Atra- } \\
\text { zine }\end{array}$ & $\begin{array}{l}\text { Buty- } \\
\text { late }\end{array}$ & $\begin{array}{l}\text { Cyana- } \\
\text { zine }\end{array}$ & $\begin{array}{l}\text { Metola- } \\
\text { chlor }\end{array}$ & $\begin{array}{l}\text { Metri- } \\
\text { buzin }\end{array}$ & $\begin{array}{l}\text { Tri- } \\
\text { flur- } \\
\text { alin }\end{array}$ \\
\hline
\end{tabular}

Site DC5, Deer Creek (fig. 2)

\begin{tabular}{|c|c|c|c|c|c|c|c|c|c|}
\hline $\begin{array}{l}10-04-89 \\
10-05-89 \\
11-08-89 \\
12-05-89 \\
01-04-90\end{array}$ & $\begin{array}{l}1145 \\
1300 \\
0800 \\
1000 \\
0900\end{array}$ & $\begin{array}{l}<0.10 \\
<.10 \\
<.10 \\
<.10 \\
<.10\end{array}$ & $\begin{array}{r}0.18 \\
<.10 \\
<.10 \\
<.10 \\
<.10\end{array}$ & $\begin{array}{l}<0.10 \\
<.10 \\
<.10 \\
<.10 \\
<.10\end{array}$ & $\begin{array}{r}0.20 \\
.36 \\
<.10 \\
<.10 \\
<.10\end{array}$ & $\begin{array}{l}<0.10 \\
<.10 \\
<.10 \\
<.10 \\
<.10\end{array}$ & $\begin{array}{l}<0 \\
< \\
< \\
< \\
<\end{array}$ & $\begin{array}{l}.10 \\
.10 \\
.10 \\
.10 \\
.10\end{array}$ & $\begin{array}{l}<0 \\
< \\
< \\
< \\
<\end{array}$ \\
\hline $\begin{array}{l}02-07-90 \\
02-08-90 \\
02-08-90 \\
03-06-90 \\
03-08-90\end{array}$ & $\begin{array}{l}1015 \\
1200 \\
1425 \\
0845 \\
2000\end{array}$ & $\begin{array}{l}<.10 \\
<.10 \\
<.10 \\
<.10 \\
<.10\end{array}$ & $\begin{aligned} & 1.4 \\
& 2.8 \\
& 2.1 \\
&<.10 \\
& 3.0\end{aligned}$ & $\begin{array}{l}<.10 \\
<.10 \\
<.10 \\
<.10 \\
<.10\end{array}$ & $\begin{array}{l}<.10 \\
<.10 \\
<.10 \\
<.10 \\
<.10\end{array}$ & $\begin{array}{l}<.10 \\
<.10 \\
.25 \\
<.10 \\
<.10\end{array}$ & $\begin{array}{l}< \\
< \\
< \\
< \\
<\end{array}$ & $\begin{array}{l}.10 \\
.10 \\
.10 \\
.10 \\
.10\end{array}$ & $\begin{array}{l}< \\
< \\
< \\
< \\
<\end{array}$ \\
\hline $\begin{array}{l}03-09-90 \\
03-16-90 \\
04-03-90 \\
05-02-90 \\
05-09-90\end{array}$ & $\begin{array}{l}1325 \\
1115 \\
0840 \\
0900 \\
0415\end{array}$ & $\begin{array}{l}<.10 \\
<.10 \\
<.10 \\
<.10 \\
<.10\end{array}$ & $\begin{aligned} & 2.3 \\
& .46 \\
&< .10 \\
&< .10 \\
& 4.7\end{aligned}$ & $\begin{array}{l}<.10 \\
<.10 \\
<.10 \\
<.10 \\
<.10\end{array}$ & $\begin{array}{l}<.10 \\
<.10 \\
<.10 \\
<.10 \\
.25\end{array}$ & $\begin{aligned}<.10 \\
<.10 \\
<.10 \\
<.10 \\
\\
2.6\end{aligned}$ & $\begin{array}{l}< \\
< \\
< \\
< \\
<\end{array}$ & $\begin{array}{l}.10 \\
.10 \\
.10 \\
.10 \\
.10\end{array}$ & $\begin{array}{l}< \\
< \\
< \\
< \\
<\end{array}$ \\
\hline $\begin{array}{l}05-09-90 \\
06-05-90 \\
06-13-90 \\
06-13-90 \\
06-22-90\end{array}$ & $\begin{array}{l}1200 \\
1045 \\
1045 \\
1115 \\
1130\end{array}$ & $\begin{array}{r}.35 \\
<.10 \\
\quad .15 \\
<.10 \\
<.10\end{array}$ & $\begin{array}{l}1.3 \\
.3 \\
47 \\
55 \\
5.2\end{array}$ & $\begin{array}{l}<.10 \\
<.10 \\
<.10 \\
<.10 \\
<.10\end{array}$ & $\begin{array}{c}<.10 \\
<.10 \\
1.4 \\
12.2 \\
1.2\end{array}$ & $\begin{array}{l}\quad .83 \\
<. .10 \\
61 \\
69 \\
3.0\end{array}$ & $\begin{array}{l}< \\
< \\
< \\
< \\
<\end{array}$ & $\begin{array}{l}.10 \\
.10 \\
.10 \\
.10 \\
.10\end{array}$ & $\begin{array}{l}< \\
< \\
< \\
< \\
<\end{array}$ \\
\hline $\begin{array}{l}06-22-90 \\
06-23-90 \\
07-02-90 \\
08-02-90 \\
08-20-90\end{array}$ & $\begin{array}{l}1445 \\
0900 \\
0900 \\
0850 \\
1410\end{array}$ & $\begin{array}{l}<.10 \\
<.10 \\
<.10 \\
<.10 \\
<.10\end{array}$ & $\begin{array}{r}2.5 \\
.93 \\
.15 \\
<.10 \\
.14\end{array}$ & $\begin{array}{l}<.10 \\
<.10 \\
<.10 \\
<.10 \\
<.10\end{array}$ & $\begin{array}{l}\quad .48 \\
<.10 \\
<.10 \\
<.10 \\
<.10\end{array}$ & $\begin{array}{r}1.1 \\
<.40 \\
<.10 \\
<.10 \\
<.10\end{array}$ & $\begin{array}{l}< \\
< \\
< \\
< \\
<\end{array}$ & $\begin{array}{l}.10 \\
.10 \\
.10 \\
.10 \\
.10\end{array}$ & $\begin{array}{l}< \\
< \\
< \\
< \\
<\end{array}$ \\
\hline $\begin{array}{l}08-26-90 \\
09-06-90\end{array}$ & $\begin{array}{l}1340 \\
0750\end{array}$ & $\begin{array}{l}<.10 \\
<.10\end{array}$ & $\begin{array}{r}.37 \\
<. .10\end{array}$ & $\begin{array}{l}<.10 \\
<.10\end{array}$ & $\begin{array}{l}<.10 \\
<.10\end{array}$ & $\begin{array}{l}<.10 \\
<.10\end{array}$ & $\begin{array}{l}< \\
<\end{array}$ & $\begin{array}{l}.10 \\
.10\end{array}$ & $<$ \\
\hline
\end{tabular}


Table 11. Selected pesticides at surface-water monitoring sites in the Big Spring basin, Clayton County, Iowa, water year 1990--Continued

\begin{tabular}{|c|c|c|c|c|c|c|c|c|}
\hline Date & $\begin{array}{c}\text { Time } \\
\text { (24-hour) }\end{array}$ & $\begin{array}{l}\text { Ala- } \\
\text { chlor }\end{array}$ & $\begin{array}{l}\text { Atra- } \\
\text { zine }\end{array}$ & $\begin{array}{l}\text { Buty- } \\
\text { late }\end{array}$ & $\begin{array}{l}\text { Cyana- } \\
\text { zine }\end{array}$ & $\begin{array}{l}\text { Metola- } \\
\text { chlor }\end{array}$ & $\begin{array}{l}\text { Metri- } \\
\text { buzin }\end{array}$ & $\begin{array}{l}\text { Tri- } \\
\text { flur- } \\
\text { alin }\end{array}$ \\
\hline
\end{tabular}

Site BOOGD Unnamed Creek near Luana (fig. 2)

$\begin{array}{lllllllll}02-06-90 & 1200 & <0.10 & 3.6 & <0.10 & 0.35 & <0.10 & <0.10 & <0.10 \\ 02-08-90 & 1300 & <.10 & 4.7 & <.10 & .41 & .46 & <.10 & <.10 \\ 02-22-90 & 1120 & .13 & 1.2 & <.10 & <.10 & <.10 & <.10 & <.10 \\ 03-08-90 & 2250 & <.10 & 5.2 & <.10 & .18 & <.10 & <.10 & <.10 \\ 06-13-90 & 1305 & 8.6 & 19 & <.10 & 2.4 & 2.1 & <.10 & <.10 \\ & & & & & & & <.1 \\ 06-22-90 & 1150 & 1.6 & 7.0 & <.10 & 1.4 & 3.8 & <.10 & <.10 \\ 06-22-90 & 1600 & 1.6 & 13 & <.10 & 7.2 & .30 & <.10 & <.10 \\ 06-23-90 & 1000 & .15 & 1.8 & <.10 & .22 & .12 & <.10 & <.10 \\ 07-02-90 & 1415 & <.10 & 1.5 & <.10 & .23 & <.10 & <.10 & <.10 \\ 07-20-90 & 1400 & <.10 & 1.2 & <.10 & .15 & <.10 & <.10 & <.10 \\ 08-01-90 & 1625 & <.10 & .57 & <.10 & <.10 & <.10 & <.10 & <.10 \\ 08-20-90 & 1215 & <.10 & .63 & <.10 & <.10 & <.10 & <.10 & <.10 \\ 08-26-90 & 1445 & <.10 & 1.4 & <.10 & <.10 & <.10 & <.10 & <.10 \\ 09-05-90 & 1540 & <.10 & .46 & <.10 & <.10 & <.10 & <.10 & <.10\end{array}$

Site RC2, Roberts Creek above Saint Olaf (fig. 2)

$\begin{array}{rrrrrrrrr}01-22-90 & 1230 & 0.44 & 3.6 & <0.10 & 0.30 & 0.47 & <0.10 & <0.10 \\ 01-31-90 & 1415 & .14 & 1.4 & <.10 & <.10 & .23 & <.10 & <.10 \\ 02-06-90 & 0930 & <.10 & 1.6 & <.10 & .35 & .23 & <.10 & <.10 \\ 02-07-90 & 1430 & <.10 & 1.9 & <.10 & <.10 & .38 & <.10 & <.10 \\ 02-08-90 & 1500 & <.10 & 2.1 & <.10 & .21 & .25 & <.10 & <.10 \\ & & & & & & .10 & .10 \\ 03-06-90 & 1300 & <.10 & 1.3 & <.10 & <.10 & <.10 & <.10 & <.10 \\ 03-08-90 & 1630 & <.10 & .81 & <.10 & .35 & <.10 & <.10 & <.10 \\ 03-08-90 & 1915 & .13 & 2.0 & <.10 & .17 & <.10 & <.10 & <.10 \\ 03-08-90 & 1950 & .18 & 2.5 & <.10 & .14 & <.10 & <.10 & <.10 \\ 03-09-90 & 0845 & .21 & 3.7 & <.10 & .27 & .34 & <.10 & <.10\end{array}$


Table 11. Selected pesticides at surface-water monitoring sites in the Big Spring basin, Clayton County, Iowa, water year 1990--Continued

\begin{tabular}{|c|c|c|c|c|c|c|c|c|}
\hline Date & $\begin{array}{c}\text { Time } \\
\text { (24-hour) }\end{array}$ & $\begin{array}{l}\text { Ala- } \\
\text { chlor }\end{array}$ & $\begin{array}{l}\text { Atra- } \\
\text { zine }\end{array}$ & $\begin{array}{l}\text { Buty- } \\
\text { late }\end{array}$ & $\begin{array}{l}\text { Cyana- } \\
\text { zine }\end{array}$ & $\begin{array}{l}\text { Metola- } \\
\text { chlor }\end{array}$ & $\begin{array}{l}\text { Metri- } \\
\text { buzin }\end{array}$ & $\begin{array}{l}\text { Tri- } \\
\text { flur- } \\
\text { alin }\end{array}$ \\
\hline & \multicolumn{8}{|c|}{ Roberts Creek } \\
\hline $\begin{array}{l}03-10-90 \\
03-12-90 \\
03-16-90 \\
04-03-90 \\
05-01-90\end{array}$ & $\begin{array}{l}0720 \\
1000 \\
1430 \\
1345 \\
1030\end{array}$ & $\begin{array}{r}0.17 \\
.40 \\
1.2 \\
<.10 \\
<.10\end{array}$ & $\begin{array}{l}3.7 \\
5.5 \\
3.9 \\
.19 \\
.21\end{array}$ & $\begin{array}{l}<0.10 \\
<.10 \\
<.10 \\
<.10 \\
<.10\end{array}$ & $\begin{array}{r}0.25 \\
.40 \\
.25 \\
<.10 \\
<.10\end{array}$ & $\begin{array}{r}0.29 \\
.97 \\
.17 \\
<.10 \\
<.10\end{array}$ & $\begin{array}{l}1.2 \\
<.10 \\
<.10 \\
<.10 \\
<.10\end{array}$ & $\begin{array}{l}<0.10 \\
<.10 \\
<.10 \\
<.10 \\
<.10\end{array}$ \\
\hline $\begin{array}{l}06-06-90 \\
06-17-90 \\
06-22-90 \\
06-22-90 \\
06-23-90\end{array}$ & $\begin{array}{l}1320 \\
1410 \\
1600 \\
1730 \\
0630\end{array}$ & $\begin{aligned} & 6.6 \\
&< .10 \\
& 1.6 \\
& 8.2 \\
& .83\end{aligned}$ & $\begin{array}{l}3.0 \\
11 \\
11 \\
12 \\
7.0\end{array}$ & $\begin{array}{l}<.10 \\
<.10 \\
<.10 \\
<.10 \\
<.10\end{array}$ & $\begin{array}{l}2.6 \\
3.4 \\
3.4 \\
8.5 \\
.46\end{array}$ & $\begin{array}{l}7.4 \\
3.4 \\
3.4 \\
<.10 \\
.20\end{array}$ & $\begin{array}{c}<.10 \\
1.6 \\
<.10 \\
<.10 \\
<.10\end{array}$ & $\begin{array}{l}<.10 \\
<.10 \\
<.10 \\
<.10 \\
<.10\end{array}$ \\
\hline $\begin{array}{l}07-03-90 \\
07-20-90 \\
08-02-90 \\
08-19-90 \\
08-20-90\end{array}$ & $\begin{array}{l}0730 \\
1000 \\
1330 \\
0255 \\
0515\end{array}$ & $\begin{array}{l}<.10 \\
<.26 \\
<.10 \\
<.10 \\
<.10\end{array}$ & $\begin{array}{c}.90 \\
.91 \\
1.4 \\
.25 \\
.16\end{array}$ & $\begin{array}{l}<.10 \\
<.10 \\
<.10 \\
<.10 \\
<.10\end{array}$ & $\begin{array}{r}.14 \\
.34 \\
.20 \\
<.10 \\
<.10\end{array}$ & $\begin{array}{l}<.10 \\
<.10 \\
<.10 \\
<.10 \\
<.10\end{array}$ & $\begin{array}{l}<.10 \\
<.10 \\
<.10 \\
<.10 \\
<.10\end{array}$ & $\begin{array}{l}<.10 \\
<.10 \\
<.10 \\
<.10 \\
<.10\end{array}$ \\
\hline $\begin{array}{l}08-25-90 \\
08-25-90 \\
08-25-90 \\
08-26-90 \\
08-26-90\end{array}$ & $\begin{array}{l}0150 \\
1045 \\
1815 \\
1015 \\
1535\end{array}$ & $\begin{array}{r}.20 \\
<.10 \\
.75 \\
.74 \\
.50\end{array}$ & $\begin{array}{l}.91 \\
1.2 \\
1.7 \\
1.7 \\
1.7\end{array}$ & $\begin{array}{l}<.10 \\
<.10 \\
<.10 \\
<.10 \\
<.10\end{array}$ & $\begin{array}{r}.11 \\
.10 \\
.22 \\
.20 \\
.19\end{array}$ & $\begin{array}{l}<.10 \\
<.10 \\
.18 \\
.17 \\
.14\end{array}$ & $\begin{array}{l}<.10 \\
<.10 \\
<.10 \\
<.10 \\
<.10\end{array}$ & $\begin{array}{l}<.10 \\
<.10 \\
<.10 \\
<.10 \\
<.10\end{array}$ \\
\hline $\begin{array}{l}08-27-90 \\
08-27-90 \\
08-28-90 \\
09-06-90\end{array}$ & $\begin{array}{l}0335 \\
1340 \\
1320 \\
1210\end{array}$ & $\begin{array}{r}.35 \\
.20 \\
<.10 \\
<.10\end{array}$ & $\begin{array}{c}1.2 \\
.94 \\
1.1 \\
.26\end{array}$ & $\begin{array}{l}<.10 \\
<.10 \\
<.10 \\
<.10\end{array}$ & $\begin{array}{l}\quad .12 \\
<.10 \\
<.10 \\
<.10\end{array}$ & $\begin{array}{l}<.10 \\
<.10 \\
<.10 \\
<.10\end{array}$ & $\begin{array}{l}<.10 \\
<.10 \\
<.10 \\
<.10\end{array}$ & $\begin{array}{l}<.10 \\
<.10 \\
<.10 \\
<.10\end{array}$ \\
\hline
\end{tabular}


Table 12. Stable isotopic ratios at sites in the Deer Creek subbasin, Clayton County, Iowa, water year 1990

[units are per mil]

\begin{tabular}{|c|c|c|}
\hline Date & $\begin{array}{l}\text { Hydrogen, } \\
2: 1 \text { ratio }\end{array}$ & $\begin{array}{l}\text { Oxygen, } \\
18: 16 \text { ratio }\end{array}$ \\
\hline \multicolumn{3}{|c|}{ Deer Creek (Site DC5, fig. 2) } \\
\hline $\begin{array}{r}10-04-89 \\
10-05-89 \\
11-08-89 \\
12-05-89 \\
5-09-90 \\
5-09-90 \\
5-09-90 \\
6-22-90 \\
6-22-90 \\
7-02-90 \\
8-02-90\end{array}$ & $\begin{array}{l}-54.0 \\
-48.5 \\
-54.5 \\
-53.0 \\
-53.5 \\
-51.5 \\
-51.0 \\
-72.0 \\
-64.0 \\
-53.5 \\
-54.0\end{array}$ & $\begin{array}{r}-8.30 \\
-7.75 \\
-8.55 \\
-8.40 \\
-8.25 \\
-8.10 \\
-8.00 \\
-10.30 \\
-9.55 \\
-8.35 \\
-8.25\end{array}$ \\
\hline \multicolumn{3}{|c|}{ Deer Creek (we11 DCW2,fig. 2) } \\
\hline $\begin{array}{r}10-04-89 \\
11-08-89 \\
12-05-89 \\
7-02-90 \\
8-02-90\end{array}$ & $\begin{array}{l}-54.5 \\
-55.0 \\
-52.0 \\
-53.0 \\
-52.0\end{array}$ & $\begin{array}{l}-8.20 \\
-8.25 \\
-8.30 \\
-8.35 \\
-8.15\end{array}$ \\
\hline \multicolumn{3}{|c|}{ Deer Creek (we11 DCW3, fig. 2) } \\
\hline $\begin{array}{r}11-08-89 \\
12-05-89 \\
7-02-90 \\
8-02-90\end{array}$ & $\begin{array}{l}-56.0 \\
-54.5 \\
-53.5 \\
-54.0\end{array}$ & $\begin{array}{l}-8.55 \\
-8.50 \\
-8.55 \\
-8.50\end{array}$ \\
\hline Deer $\mathrm{Cr}$ & (tilelin & OCT2, fig. 2) \\
\hline $\begin{array}{l}7-02-90 \\
8-02-90\end{array}$ & $\begin{array}{l}-49.5 \\
-50.5\end{array}$ & $\begin{array}{l}-8.05 \\
-7.75\end{array}$ \\
\hline
\end{tabular}


Table 13. Daily mean discharge at site L23S, Silver Creek near Luana, Iowa, water year 1990

[Discharge in cubic feet per second]

\begin{tabular}{|c|c|c|c|c|c|c|c|c|c|c|c|c|}
\hline Day & Oct. & Nov. & Dec. & Jan. & Feb. & Mar. & Apr. & May & June & July & Aus. & Sept. \\
\hline $\begin{array}{l}1 \\
2 \\
3 \\
4 \\
5\end{array}$ & $\begin{array}{l}0.07 \\
.07 \\
.07 \\
.04 \\
.22\end{array}$ & $\begin{array}{r}0.22 \\
.19 \\
.16 \\
.17 \\
.17\end{array}$ & $\begin{array}{r}0.07 \\
.09 \\
.03 \\
.08 \\
.15\end{array}$ & $\begin{array}{l}0 \\
0 \\
0 \\
0 \\
0\end{array}$ & $\begin{array}{l}0 \\
0 \\
0 \\
0 \\
.02\end{array}$ & $\begin{array}{r}0.70 \\
.50 \\
.35 \\
.26 \\
.20\end{array}$ & $\begin{array}{r}0.24 \\
.27 \\
.23 \\
.25 \\
.25\end{array}$ & $\begin{array}{r}0.25 \\
.25 \\
.25 \\
.30 \\
.30\end{array}$ & $\begin{array}{l}0.56 \\
5.1 \\
1.8 \\
.79 \\
.70\end{array}$ & $\begin{array}{l}1.4 \\
1.3 \\
1.2 \\
1.2 \\
1.1\end{array}$ & $\begin{array}{r}0.92 \\
.88 \\
.93 \\
23 \\
2.3\end{array}$ & $\begin{array}{l}2.8 \\
2.4 \\
2.4 \\
2.4 \\
2.2\end{array}$ \\
\hline $\begin{array}{r}6 \\
7 \\
8 \\
9 \\
10\end{array}$ & $\begin{array}{l}.13 \\
.09 \\
.08 \\
.09 \\
.10\end{array}$ & $\begin{array}{l}.17 \\
.17 \\
.16 \\
.11 \\
.10\end{array}$ & $\begin{array}{l}.12 \\
.06 \\
.04 \\
.03 \\
.03\end{array}$ & $\begin{array}{l}0 \\
0 \\
.01 \\
.02 \\
.02\end{array}$ & $\begin{array}{r}.10 \\
3.0 \\
.80 \\
.25 \\
.15\end{array}$ & $\begin{array}{r}.13 \\
55 \\
.12 \\
2.9 \\
.89\end{array}$ & $\begin{array}{l}.20 \\
.18 \\
.21 \\
.26 \\
.40\end{array}$ & $\begin{array}{l}.30 \\
.28 \\
.25 \\
.52 \\
.46\end{array}$ & $\begin{array}{l}.67 \\
.61 \\
.60 \\
.56 \\
.52\end{array}$ & $\begin{array}{l}.99 \\
.97 \\
.97 \\
.97 \\
.97\end{array}$ & $\begin{array}{l}1.5 \\
1.3 \\
1.3 \\
1.1 \\
1.1\end{array}$ & $\begin{array}{l}1.6 \\
1.5 \\
1.5 \\
1.5 \\
1.4\end{array}$ \\
\hline $\begin{array}{l}11 \\
12 \\
13 \\
14 \\
15\end{array}$ & $\begin{array}{l}.10 \\
.09 \\
.08 \\
.08 \\
.10\end{array}$ & $\begin{array}{l}.10 \\
.10 \\
.10 \\
.10 \\
.10\end{array}$ & $\begin{array}{l}0^{.02} \\
0 \\
0 \\
0\end{array}$ & $\begin{array}{l}0^{.01} \\
0 \\
0 \\
0\end{array}$ & $\begin{array}{l}.06 \\
.13 \\
.08 \\
.06 \\
.04\end{array}$ & $\begin{array}{c}30 \\
3.0 \\
2.6 \\
1.5 \\
.81\end{array}$ & $\begin{array}{l}.32 \\
.27 \\
.27 \\
.27 \\
.27\end{array}$ & $\begin{array}{l}.38 \\
.35 \\
.35 \\
.35 \\
.35\end{array}$ & $\begin{array}{l}.52 \\
1.3 \\
6.3 \\
1.0 \\
3.1\end{array}$ & $\begin{array}{l}.97 \\
.97 \\
.94 \\
.88 \\
.88\end{array}$ & $\begin{array}{r}1.1 \\
.98 \\
.90 \\
.88 \\
.88\end{array}$ & $\begin{array}{l}1.4 \\
1.3 \\
1.1 \\
1.1 \\
1.1\end{array}$ \\
\hline $\begin{array}{l}16 \\
17 \\
18 \\
19 \\
20\end{array}$ & $\begin{array}{l}.30 \\
.16 \\
.14 \\
.14 \\
.14\end{array}$ & $\begin{array}{l}.07 \\
.07 \\
.06 \\
.08 \\
.11\end{array}$ & $\begin{array}{l}0 \\
0 \\
0 \\
0 \\
0\end{array}$ & $\begin{array}{l}.01 \\
.03 \\
.02 \\
.01 \\
.01\end{array}$ & $\begin{array}{l}.03 \\
.02 \\
.03 \\
.02 \\
.02\end{array}$ & $\begin{array}{l}.49 \\
.40 \\
.26 \\
.24 \\
.25\end{array}$ & $\begin{array}{l}.27 \\
.28 \\
.30 \\
.30 \\
.30\end{array}$ & $\begin{array}{r}.41 \\
.41 \\
.41 \\
2.3 \\
2.1\end{array}$ & $\begin{array}{l}2.2 \\
4.8 \\
2.0 \\
1.3 \\
1.2\end{array}$ & $\begin{array}{c}.88 \\
.83 \\
1.3 \\
3.6 \\
2.6\end{array}$ & $\begin{array}{r}.81 \\
.94 \\
1.0 \\
4.5 \\
3.4\end{array}$ & $\begin{array}{l}.98 \\
.89 \\
.88 \\
.88 \\
.88\end{array}$ \\
\hline $\begin{array}{l}21 \\
22 \\
23 \\
24 \\
25\end{array}$ & $\begin{array}{l}.14 \\
.12 \\
.12 \\
.11 \\
.09\end{array}$ & $\begin{array}{l}.07 \\
.08 \\
.08 \\
.07 \\
.08\end{array}$ & $\begin{array}{l}0 \\
0 \\
0 \\
0 \\
0\end{array}$ & $\begin{array}{l}.02 \\
.02 \\
.01 \\
0 \\
0\end{array}$ & $\begin{array}{l}.01 \\
.02 \\
.03 \\
.02 \\
.02\end{array}$ & $\begin{array}{l}.27 \\
.33 \\
.25 \\
.22 \\
.26\end{array}$ & $\begin{array}{l}.30 \\
.30 \\
.29 \\
.27 \\
.26\end{array}$ & $\begin{array}{c}1.0 \\
.81 \\
.74 \\
.71 \\
.64\end{array}$ & $\begin{array}{l}.99 \\
6.4 \\
5.5 \\
4.1 \\
3.0\end{array}$ & $\begin{array}{l}1.4 \\
1.3 \\
1.2 \\
1.2 \\
1.2\end{array}$ & $\begin{array}{r}1.9 \\
1.3 \\
1.2 \\
5.4 \\
107\end{array}$ & $\begin{array}{l}.88 \\
.88 \\
.88 \\
.81 \\
.77\end{array}$ \\
\hline $\begin{array}{l}26 \\
27 \\
28 \\
29 \\
30 \\
31\end{array}$ & $\begin{array}{l}.10 \\
.10 \\
.10 \\
.10 \\
.28 \\
.31\end{array}$ & $\begin{array}{l}.08 \\
.11 \\
.07 \\
.04 \\
.04\end{array}$ & $\begin{array}{l}0 \\
0 \\
0 \\
0 \\
0 \\
0\end{array}$ & $\begin{array}{l}0 \\
0 \\
0 \\
0 \\
0 \\
0\end{array}$ & $\begin{array}{l}.01 \\
.02 \\
.04\end{array}$ & $\begin{array}{l}.25 \\
.22 \\
.22 \\
.22 \\
.22 \\
.22\end{array}$ & $\begin{array}{l}.25 \\
.27 \\
.29 \\
.30 \\
.27\end{array}$ & $\begin{array}{l}.62 \\
.58 \\
.64 \\
.64 \\
.60 \\
.56\end{array}$ & $\begin{array}{l}2.4 \\
2.1 \\
1.8 \\
1.6 \\
1.5\end{array}$ & $\begin{array}{c}1.2 \\
3.0 \\
1.9 \\
1.5 \\
1.4 \\
.96\end{array}$ & $\begin{array}{c}15 \\
8.3 \\
6.0 \\
5.1 \\
4.1 \\
3.4\end{array}$ & $\begin{array}{l}.74 \\
.74 \\
.74 \\
.72 \\
.69\end{array}$ \\
\hline
\end{tabular}


Table 14. Daily mean discharge at site BOOGD, Unnamed Creek near Luana, Iowa, water year 1990

[Discharge in cubic feet per second]

\begin{tabular}{|c|c|c|c|c|c|c|c|c|c|c|c|c|}
\hline Day & Oct. & Nov. & Dec. & Jan. & Feb. & Mar. & Apr. & May & June & July & Aug. & Sept. \\
\hline $\begin{array}{l}1 \\
2 \\
3 \\
4 \\
5\end{array}$ & $\begin{array}{l}0 \\
0 \\
0 \\
0 \\
\\
\\
.01\end{array}$ & $\begin{array}{l}0 \\
0 \\
0 \\
0 \\
0\end{array}$ & $\begin{array}{l}0 \\
0 \\
0 \\
0 \\
0\end{array}$ & $\begin{array}{l}0 \\
0 \\
0 \\
0 \\
0\end{array}$ & $\begin{array}{l}0 \\
0 \\
0 \\
0 \\
.07\end{array}$ & $\begin{array}{r}0.25 \\
.15 \\
.11 \\
.08 \\
.05\end{array}$ & $\begin{array}{l}0 \\
0 \\
0 \\
0 \\
0\end{array}$ & $\begin{array}{l}0 \\
0 \\
0 \\
0 \\
0\end{array}$ & $\begin{array}{l}0 \\
.01 \\
0 \\
0 \\
0\end{array}$ & $\begin{array}{r}0.15 \\
.09 \\
.08 \\
.08 \\
.07\end{array}$ & $\begin{array}{r}0.09 \\
.04 \\
.06 \\
4.7 \\
.07\end{array}$ & $\begin{array}{r}0.94 \\
.80 \\
.80 \\
.94 \\
.86\end{array}$ \\
\hline $\begin{array}{r}6 \\
7 \\
8 \\
9 \\
10\end{array}$ & $\begin{array}{l}0 \\
0 \\
0 \\
0 \\
0\end{array}$ & $\begin{array}{l}0 \\
0 \\
0 \\
0 \\
0\end{array}$ & $\begin{array}{l}0 \\
0 \\
0 \\
0 \\
0\end{array}$ & $\begin{array}{l}0 \\
0 \\
0 \\
0 \\
0\end{array}$ & $\begin{array}{l}.22 \\
.4 \\
.52 \\
.3 \\
.15\end{array}$ & $\begin{array}{l}.02 \\
0^{.02} \\
.32 \\
.04\end{array}$ & $\begin{array}{l}0 \\
0 \\
0 \\
0 \\
0\end{array}$ & $\begin{array}{l}0 \\
0 \\
0 \\
0 \\
0\end{array}$ & $\begin{array}{l}0 \\
0 \\
0 \\
0 \\
0\end{array}$ & $\begin{array}{l}.23 \\
.20 \\
.13 \\
.24 \\
.17\end{array}$ & $\begin{array}{r}.04 \\
.05 \\
.07 \\
.52 \\
1.2\end{array}$ & $\begin{array}{l}.35 \\
.24 \\
.17 \\
.28 \\
.28\end{array}$ \\
\hline $\begin{array}{l}11 \\
12 \\
13 \\
14 \\
15\end{array}$ & $\begin{array}{l}0 \\
0 \\
0 \\
0 \\
0\end{array}$ & $\begin{array}{l}0 \\
0 \\
0 \\
0 \\
0\end{array}$ & $\begin{array}{l}0 \\
0 \\
0 \\
0 \\
0\end{array}$ & $\begin{array}{l}0 \\
0 \\
0 \\
0 \\
0\end{array}$ & $\begin{array}{l}.1 \\
.13 \\
.09 \\
.06 \\
.04\end{array}$ & $\begin{array}{l}4.7 \\
1.4 \\
.89 \\
.23 \\
.04\end{array}$ & $\begin{array}{l}0 \\
0 \\
0 \\
0 \\
0\end{array}$ & $\begin{array}{l}0 \\
0 \\
0 \\
0 \\
0\end{array}$ & $\begin{array}{l}0 \\
0 \\
1.0 \\
.05 \\
.57\end{array}$ & $\begin{array}{l}.07 \\
.11 \\
.08 \\
.07 \\
.06\end{array}$ & $\begin{array}{l}1.1 \\
1.0 \\
.79 \\
.48 \\
.17\end{array}$ & $\begin{array}{l}.16 \\
.15 \\
.14 \\
.12 \\
.13\end{array}$ \\
\hline $\begin{array}{l}16 \\
17 \\
18 \\
19 \\
20\end{array}$ & $\begin{array}{l}0^{.01} \\
0 \\
0 \\
0\end{array}$ & $\begin{array}{l}0 \\
0 \\
0 \\
0 \\
0\end{array}$ & $\begin{array}{l}0 \\
0 \\
0 \\
0 \\
0\end{array}$ & $\begin{array}{l}.02 \\
0.01 \\
0 \\
0\end{array}$ & $\begin{array}{l}.02 \\
.01 \\
.03 \\
.02 \\
.02\end{array}$ & $\begin{array}{l}0 \\
0 \\
0 \\
0 \\
0\end{array}$ & $\begin{array}{l}0 \\
0 \\
0 \\
0 \\
0\end{array}$ & $\begin{array}{l}0 \\
0 \\
0 \\
0 \\
0\end{array}$ & $\begin{array}{l}1.0 \\
.62 \\
.11 \\
.10 \\
.11\end{array}$ & $\begin{array}{l}.07 \\
.05 \\
.12 \\
.59 \\
.11\end{array}$ & $\begin{array}{r}.10 \\
.54 \\
.40 \\
1.6 \\
.46\end{array}$ & $\begin{array}{l}.11 \\
.09 \\
.08 \\
.09 \\
.12\end{array}$ \\
\hline $\begin{array}{l}21 \\
22 \\
23 \\
24 \\
25\end{array}$ & $\begin{array}{l}0 \\
0 \\
0 \\
0 \\
0\end{array}$ & $\begin{array}{l}0 \\
0 \\
0 \\
0 \\
0\end{array}$ & $\begin{array}{l}0 \\
0 \\
0 \\
0 \\
0\end{array}$ & $\begin{array}{l}0 \\
.02 \\
.01 \\
0 \\
0\end{array}$ & $\begin{array}{l}.02 \\
.03 \\
.02 \\
.01 \\
.01\end{array}$ & $\begin{array}{l}0 \\
0 \\
0 \\
0 \\
0\end{array}$ & $\begin{array}{l}0 \\
0 \\
0 \\
0 \\
0\end{array}$ & $\begin{array}{l}0 \\
0 \\
0 \\
0\end{array}$ & $\begin{array}{c}.07 \\
1.7 \\
.09 \\
.09 \\
.07\end{array}$ & $\begin{array}{l}.08 \\
.06 \\
.06 \\
.06 \\
.11\end{array}$ & $\begin{array}{r}.24 \\
.19 \\
.34 \\
1.9 \\
21\end{array}$ & $\begin{array}{l}.08 \\
.06 \\
.06 \\
.05 \\
.06\end{array}$ \\
\hline $\begin{array}{l}26 \\
27 \\
28 \\
29 \\
30 \\
31\end{array}$ & $\begin{array}{l}0 \\
0 \\
0 \\
0 \\
0^{.01}\end{array}$ & $\begin{array}{l}0 \\
0 \\
0 \\
0 \\
0\end{array}$ & $\begin{array}{l}0 \\
0 \\
0 \\
0 \\
0 \\
0\end{array}$ & $\begin{array}{l}0 \\
0 \\
0 \\
0 \\
0 \\
0\end{array}$ & $\begin{array}{r}0 \\
.02 \\
.03\end{array}$ & $\begin{array}{l}0 \\
0 \\
0 \\
0 \\
0 \\
0\end{array}$ & $\begin{array}{l}0 \\
0 \\
0 \\
0 \\
0\end{array}$ & $\begin{array}{l}0 \\
0 \\
0 \\
0 \\
0 \\
.01\end{array}$ & $\begin{array}{l}.08 \\
.08 \\
.08 \\
.09 \\
.08\end{array}$ & $\begin{array}{l}.08 \\
.27 \\
.27 \\
.23 \\
.38 \\
.25\end{array}$ & $\begin{array}{l}9.1 \\
3.0 \\
2.2 \\
1.6 \\
1.4 \\
1.0\end{array}$ & $\begin{array}{l}.15 \\
.22 \\
.07 \\
.07 \\
.07\end{array}$ \\
\hline
\end{tabular}


Table 15. Daily mean suspended-sediment concentrations and daily suspended-sediment load at site BOOGD, Unnamed Creek near Luana, Iowa, water year 1990

\begin{tabular}{|c|c|c|c|c|c|c|c|c|c|c|c|c|}
\hline Day & Oct. & Nov. & Dec. & Jan. & Feb. & Mar. & Apr. & May & June & July & Aug. & Sept \\
\hline \multicolumn{13}{|c|}{ Daily mean suspended-sediment concentration, in milligrams per liter } \\
\hline $\begin{array}{l}1 \\
2 \\
3 \\
4 \\
5\end{array}$ & $\begin{array}{l}--- \\
=-- \\
--- \\
---\end{array}$ & $\begin{array}{l}--- \\
=-- \\
--- \\
---\end{array}$ & $\begin{array}{l}--- \\
=-- \\
--- \\
---\end{array}$ & $\begin{array}{l}--- \\
=-- \\
--- \\
---\end{array}$ & $\begin{array}{l}--- \\
=-- \\
=-- \\
---\end{array}$ & $\begin{array}{l}192 \\
649 \\
405 \\
383 \\
382\end{array}$ & $\begin{array}{l}--- \\
--- \\
--- \\
---\end{array}$ & $\begin{array}{l}--- \\
=-- \\
--: \\
---\end{array}$ & $\begin{array}{l}--- \\
-- \\
-- \\
---\end{array}$ & $\begin{array}{l}13 \\
12 \\
13 \\
13 \\
13\end{array}$ & $\begin{array}{l}25 \\
25 \\
25 \\
24 \\
24\end{array}$ & $\begin{array}{r}27 \\
26 \\
25 \\
26 \\
575\end{array}$ \\
\hline $\begin{array}{r}6 \\
7 \\
8 \\
9 \\
10\end{array}$ & $\begin{array}{l}--- \\
--- \\
--- \\
---\end{array}$ & $\begin{array}{l}--- \\
--- \\
--- \\
---\end{array}$ & $\begin{array}{l}--- \\
--- \\
--- \\
--- \\
---\end{array}$ & $\begin{array}{l}--- \\
--- \\
--- \\
---\end{array}$ & $\begin{array}{r}--- \\
354 \\
168 \\
81\end{array}$ & $\begin{array}{r}417 \\
394 \\
479 \\
98 \\
10\end{array}$ & $\begin{array}{l}--- \\
=-- \\
--- \\
---\end{array}$ & $\begin{array}{l}=-- \\
=-- \\
=-- \\
--\end{array}$ & $\begin{array}{l}--- \\
--- \\
--- \\
---\end{array}$ & $\begin{array}{l}14 \\
14 \\
14 \\
15 \\
15\end{array}$ & $\begin{array}{r}23 \\
24 \\
27 \\
879 \\
736\end{array}$ & $\begin{array}{l}581 \\
250 \\
277 \\
299 \\
396\end{array}$ \\
\hline $\begin{array}{l}11 \\
12 \\
13 \\
14 \\
15\end{array}$ & $\begin{array}{l}--- \\
--- \\
--- \\
---\end{array}$ & $\begin{array}{l}--- \\
--- \\
--- \\
--- \\
---\end{array}$ & $\begin{array}{l}--- \\
--- \\
--- \\
--- \\
---\end{array}$ & $\begin{array}{l}--- \\
--- \\
--- \\
--- \\
---\end{array}$ & $\begin{array}{r}81 \\
498 \\
252 \\
40 \\
37\end{array}$ & $\begin{array}{l}689 \\
119 \\
696 \\
240 \\
---\end{array}$ & $\begin{array}{l}--- \\
-- \\
-- \\
--- \\
--\end{array}$ & $\begin{array}{l}\because-- \\
\because-- \\
\because-- \\
--\end{array}$ & $\begin{array}{r}--- \\
\overline{1,140} \\
1,130 \\
1,140\end{array}$ & $\begin{array}{r}15 \\
15 \\
16 \\
384 \\
139\end{array}$ & $\begin{array}{l}963 \\
642 \\
772 \\
413 \\
824\end{array}$ & $\begin{array}{r}742 \\
242 \\
23 \\
9 \\
10\end{array}$ \\
\hline $\begin{array}{l}16 \\
17 \\
18 \\
19 \\
20\end{array}$ & $\begin{array}{l}--- \\
=-- \\
--- \\
---\end{array}$ & $\begin{array}{l}--- \\
=-- \\
=-- \\
---\end{array}$ & $\begin{array}{l}--- \\
--- \\
--- \\
--- \\
---\end{array}$ & $\begin{array}{r}227 \\
240 \\
162 \\
82 \\
53\end{array}$ & $\begin{array}{l}40 \\
60 \\
51 \\
47 \\
49\end{array}$ & $\begin{array}{l}--- \\
-- \\
--- \\
--- \\
--\end{array}$ & $\begin{array}{l}--- \\
\overline{--} \\
--- \\
---\end{array}$ & $\begin{array}{l}--- \\
=-- \\
--- \\
---\end{array}$ & $\begin{array}{l}414 \\
-- \\
55 \\
450\end{array}$ & $\begin{array}{l}138 \\
316 \\
775 \\
368 \\
447\end{array}$ & $\begin{array}{r}160 \\
40 \\
39 \\
39 \\
38\end{array}$ & $\begin{array}{l}14 \\
15 \\
13 \\
12 \\
11\end{array}$ \\
\hline $\begin{array}{l}21 \\
22 \\
23 \\
24 \\
25\end{array}$ & $\begin{array}{l}--- \\
-- \\
-- \\
---\end{array}$ & $\begin{array}{l}=-- \\
=-- \\
=-- \\
---\end{array}$ & $\begin{array}{l}=-- \\
=-- \\
=-- \\
---\end{array}$ & $\begin{array}{r}44 \\
42 \\
39 \\
--- \\
---\end{array}$ & $\begin{array}{r}47 \\
-34 \\
32 \\
30\end{array}$ & $\begin{array}{l}--- \\
-- \\
--- \\
--- \\
--\end{array}$ & $\begin{array}{l}--- \\
--- \\
--- \\
--- \\
--\end{array}$ & $\begin{array}{l}=- \\
=-- \\
=-- \\
---\end{array}$ & $\begin{array}{r}407 \\
689 \\
256 \\
198 \\
64\end{array}$ & $\begin{array}{r}597 \\
37 \\
26 \\
26 \\
26\end{array}$ & $\begin{array}{l}36 \\
35 \\
34 \\
34 \\
33\end{array}$ & $\begin{array}{l}11 \\
11 \\
12 \\
12 \\
13\end{array}$ \\
\hline $\begin{array}{l}26 \\
27 \\
28 \\
29 \\
30 \\
31\end{array}$ & $\begin{array}{l}--- \\
=-- \\
=-- \\
--- \\
---\end{array}$ & $\begin{array}{l}--- \\
=-- \\
--- \\
---\end{array}$ & $\begin{array}{l}--- \\
--- \\
--- \\
--- \\
---\end{array}$ & $\begin{array}{l}--- \\
=-- \\
--- \\
--- \\
---\end{array}$ & $\begin{array}{r}--- \\
28 \\
29\end{array}$ & $\begin{array}{l}--- \\
--- \\
--- \\
--- \\
---\end{array}$ & $\begin{array}{l}--- \\
--- \\
--- \\
---\end{array}$ & $\begin{array}{l}=- \\
=-- \\
--- \\
--- \\
--\end{array}$ & $\begin{array}{l}19 \\
10 \\
10 \\
12 \\
13\end{array}$ & $\begin{array}{l}25 \\
24 \\
24 \\
24 \\
24 \\
25\end{array}$ & $\begin{array}{l}32 \\
32 \\
31 \\
30 \\
30 \\
29\end{array}$ & $\begin{array}{l}13 \\
12 \\
13 \\
15 \\
15\end{array}$ \\
\hline
\end{tabular}

Daily suspended-sediment load, in tons

\begin{tabular}{|c|c|c|c|c|c|c|c|c|c|c|c|c|}
\hline $\begin{array}{l}1 \\
2 \\
3 \\
4 \\
5\end{array}$ & $\begin{array}{l}0 \\
0 \\
0 \\
0 \\
0\end{array}$ & $\begin{array}{l}0 \\
0 \\
0 \\
0 \\
0\end{array}$ & $\begin{array}{l}0 \\
0 \\
0 \\
0 \\
0\end{array}$ & $\begin{array}{l}0 \\
0 \\
0 \\
0 \\
0\end{array}$ & $\begin{array}{l}0 \\
0 \\
0 \\
0 \\
0\end{array}$ & $\begin{array}{r}0.13 \\
.26 \\
.12 \\
.08 \\
.05\end{array}$ & $\begin{array}{l}0 \\
0 \\
0 \\
0 \\
0\end{array}$ & $\begin{array}{l}0 \\
0 \\
0 \\
0 \\
0\end{array}$ & $\begin{array}{l}0 \\
0 \\
0 \\
0 \\
0\end{array}$ & $\begin{array}{l}0.01 \\
0 \\
0 \\
0 \\
0\end{array}$ & $\begin{array}{l}0.01 \\
0 \\
0 \\
0^{.31}\end{array}$ & $\begin{array}{r}0.07 \\
.06 \\
.05 \\
.07 \\
1.3\end{array}$ \\
\hline $\begin{array}{r}6 \\
7 \\
8 \\
9 \\
10\end{array}$ & $\begin{array}{l}0 \\
0 \\
0 \\
0 \\
0\end{array}$ & $\begin{array}{l}0 \\
0 \\
0 \\
0 \\
0\end{array}$ & $\begin{array}{l}0 \\
0 \\
0 \\
0 \\
0\end{array}$ & $\begin{array}{l}0 \\
0 \\
0 \\
0 \\
0\end{array}$ & $\begin{array}{l}--- \\
--.5 \\
.50 \\
.14 \\
.03\end{array}$ & $\begin{array}{l}0^{.02} \\
3.2 \\
0^{.08}\end{array}$ & $\begin{array}{l}0 \\
0 \\
0 \\
0 \\
0\end{array}$ & $\begin{array}{l}0 \\
0 \\
0 \\
0 \\
0\end{array}$ & $\begin{array}{l}0 \\
0 \\
0 \\
0 \\
0\end{array}$ & $\begin{array}{l}.01 \\
.01 \\
.01 \\
.01 \\
.01\end{array}$ & $\begin{array}{l}0 \\
0 \\
1.01 \\
2.2\end{array}$ & $\begin{array}{l}.55 \\
.16 \\
.13 \\
.23 \\
.30\end{array}$ \\
\hline 1 & $\begin{array}{l}0 \\
0 \\
0 \\
0 \\
0\end{array}$ & $\begin{array}{l}0 \\
0 \\
0 \\
0 \\
0\end{array}$ & $\begin{array}{l}0 \\
0 \\
0 \\
0 \\
0\end{array}$ & $\begin{array}{l}0 \\
0 \\
0 \\
0 \\
0\end{array}$ & $\begin{array}{r}.02 \\
.17 \\
.06 \\
.01 \\
0^{.01}\end{array}$ & $\begin{array}{l}8.7 \\
1.45 \\
0.75 \\
0.15\end{array}$ & $\begin{array}{l}0 \\
0 \\
0 \\
0 \\
0\end{array}$ & $\begin{array}{l}0 \\
0 \\
0 \\
0 \\
0\end{array}$ & $\begin{array}{l}0 \\
0 \\
3.1 \\
.15 \\
1.8\end{array}$ & $\begin{array}{l}0 \\
0 \\
0 \\
.07 \\
.02\end{array}$ & $\begin{array}{c}2.9 \\
1.7 \\
1.6 \\
.54 \\
.38\end{array}$ & $\begin{array}{l}.32 \\
.10 \\
.01 \\
0^{.01} \\
0\end{array}$ \\
\hline 16 & $\begin{array}{l}0 \\
0 \\
0 \\
0 \\
0\end{array}$ & $\begin{array}{l}0 \\
0 \\
0 \\
0 \\
0\end{array}$ & $\begin{array}{l}0 \\
0 \\
0 \\
0 \\
0\end{array}$ & $\begin{array}{l}.01 \\
0^{.01} \\
0 \\
0\end{array}$ & $\begin{array}{l}0 \\
0 \\
0 \\
0 \\
0\end{array}$ & $\begin{array}{l}0 \\
0 \\
0 \\
0 \\
0\end{array}$ & $\begin{array}{l}0 \\
0 \\
0 \\
0 \\
0\end{array}$ & $\begin{array}{l}0 \\
0 \\
0 \\
0 \\
0\end{array}$ & $\begin{array}{c}1.1 \\
-.- \\
-01 \\
.01\end{array}$ & $\begin{array}{l}.03 \\
.04 \\
.25 \\
.59 \\
.13\end{array}$ & $\begin{array}{l}.04 \\
.06 \\
.04 \\
.17 \\
.05\end{array}$ & $\begin{array}{l}0 \\
0 \\
0 \\
0 \\
0\end{array}$ \\
\hline 2 & $\begin{array}{l}0 \\
0 \\
0 \\
0 \\
0\end{array}$ & $\begin{array}{l}0 \\
0 \\
0 \\
0 \\
0\end{array}$ & $\begin{array}{l}0 \\
0 \\
0 \\
0 \\
0\end{array}$ & $\begin{array}{l}0 \\
0 \\
0 \\
0 \\
0\end{array}$ & $\begin{array}{l}0 \\
0_{0}^{---} \\
0 \\
0\end{array}$ & $\begin{array}{l}0 \\
0 \\
0 \\
0 \\
0\end{array}$ & $\begin{array}{l}0 \\
0 \\
0 \\
0 \\
0\end{array}$ & $\begin{array}{l}0 \\
0 \\
0 \\
0 \\
0\end{array}$ & $\begin{array}{r}.08 \\
3.2 \\
.06 \\
.05 \\
.01\end{array}$ & $\begin{array}{l}.13 \\
0_{0}^{.01} \\
.01\end{array}$ & $\begin{array}{r}.02 \\
.02 \\
.03 \\
.17 \\
1.9\end{array}$ & $\begin{array}{l}0 \\
0 \\
0 \\
0 \\
0\end{array}$ \\
\hline $\begin{array}{l}27 \\
28 \\
29\end{array}$ & $\begin{array}{l}0 \\
0 \\
0 \\
0 \\
0 \\
0\end{array}$ & $\begin{array}{l}0 \\
0 \\
0 \\
0 \\
0\end{array}$ & $\begin{array}{l}0 \\
0 \\
0 \\
0 \\
0 \\
0\end{array}$ & $\begin{array}{l}0 \\
0 \\
0 \\
0 \\
0 \\
0\end{array}$ & $\begin{array}{l}0 \\
0 \\
0\end{array}$ & $\begin{array}{l}0 \\
0 \\
0 \\
0 \\
0 \\
0\end{array}$ & $\begin{array}{l}0 \\
0 \\
0 \\
0 \\
0\end{array}$ & $\begin{array}{l}0 \\
0 \\
0 \\
0 \\
0 \\
0\end{array}$ & $\begin{array}{l}0 \\
0 \\
0 \\
0 \\
0\end{array}$ & $\begin{array}{l}.01 \\
.02 \\
.02 \\
.01 \\
.02 \\
.02\end{array}$ & $\begin{array}{l}.79 \\
.26 \\
.18 \\
.13 \\
.11 \\
.09\end{array}$ & $\begin{array}{l}.01 \\
0.01 \\
0 \\
0\end{array}$ \\
\hline $\begin{array}{l}\text { ured } \\
\text { und }\end{array}$ & 0.00 & 0.00 & 0.00 & 0.02 & 0.93 & 14.94 & 0.00 & 0.00 & 9.69 & 1.44 & 15.11 & 3.37 \\
\hline
\end{tabular}


Table 16. Daily mean discharge and specific conductance at site RC2, Roberts Creek above Saint Olaf, Iowa, water year 1990

[---, data not available to calculate mean values; water-quality instrumentation was removed from November 27 to March 20]

\begin{tabular}{|c|c|c|c|c|c|c|c|c|c|c|c|c|}
\hline Day & Oct. & Nov. & Dec. & Jan. & Feb. & Mar. & Apr. & May & June & July & Aus. & Sept. \\
\hline \multicolumn{13}{|c|}{ Daily mean discharge, in cubic feet per second } \\
\hline $\begin{array}{l}1 \\
2 \\
3 \\
4 \\
5\end{array}$ & $\begin{array}{r}0.14 \\
.04 \\
.04 \\
.07 \\
.23\end{array}$ & $\begin{array}{l}0.09 \\
0 \\
0 \\
0 \\
0\end{array}$ & $\begin{array}{l}0 \\
0 \\
0 \\
0 \\
0\end{array}$ & $\begin{array}{l}0 \\
0 \\
0 \\
0 \\
0\end{array}$ & $\begin{array}{r}0.05 \\
.04 \\
.10 \\
.60 \\
2.0\end{array}$ & $\begin{array}{l}1.8 \\
2.0 \\
2.5 \\
3.0 \\
2.0\end{array}$ & $\begin{array}{l}2.6 \\
2.5 \\
2.5 \\
2.5 \\
2.4\end{array}$ & $\begin{array}{r}0.77 \\
.76 \\
.64 \\
.71 \\
.82\end{array}$ & $\begin{array}{r}2.8 \\
5.2 \\
16 \\
8.5 \\
6.6\end{array}$ & $\begin{array}{r}11 \\
8.8 \\
7.6 \\
7.6 \\
6.4\end{array}$ & $\begin{array}{r}6.6 \\
6.0 \\
5.6 \\
5.2 \\
19\end{array}$ & $\begin{array}{l}20 \\
18 \\
16 \\
15 \\
15\end{array}$ \\
\hline $\begin{array}{r}6 \\
7 \\
8 \\
9 \\
10\end{array}$ & $\begin{array}{l}.18 \\
.11 \\
.06 \\
.07 \\
.05\end{array}$ & $\begin{array}{l}0 \\
0 \\
0 \\
0 \\
0\end{array}$ & $\begin{array}{l}0 \\
0 \\
0 \\
0 \\
0\end{array}$ & $\begin{array}{l}0 \\
0 \\
0 \\
0 \\
.05\end{array}$ & $\begin{array}{c}30 \\
27 \\
35 \\
17 \\
8.0\end{array}$ & $\begin{array}{c}1.6 \\
1.4 \\
35 \\
187 \\
35\end{array}$ & $\begin{array}{l}2.1 \\
1.9 \\
1.9 \\
2.0 \\
3.0\end{array}$ & $\begin{array}{l}.88 \\
.79 \\
.65 \\
1.1 \\
2.4\end{array}$ & $\begin{array}{l}6.6 \\
5.7 \\
5.0 \\
4.2 \\
3.6\end{array}$ & $\begin{array}{l}5.5 \\
4.5 \\
5.3 \\
4.1 \\
3.3\end{array}$ & $\begin{array}{l}8.1 \\
5.9 . \\
5.4 \\
4.9 \\
4.5\end{array}$ & $\begin{array}{c}13 \\
12 \\
10 \\
9.3 \\
8.4\end{array}$ \\
\hline $\begin{array}{l}11 \\
12 \\
13 \\
14 \\
15\end{array}$ & $\begin{array}{l}.11 \\
.09 \\
.06 \\
.05 \\
.08\end{array}$ & $\begin{array}{l}0 \\
0 \\
0 \\
0 \\
0\end{array}$ & $\begin{array}{l}0 \\
0 \\
0 \\
0 \\
0\end{array}$ & $\begin{array}{l}.10 \\
.05 \\
0_{0}^{.01}\end{array}$ & $\begin{array}{l}6.0 \\
8.0 \\
6.0 \\
4.0 \\
3.0\end{array}$ & $\begin{array}{r}175 \\
80 \\
39 \\
97 \\
41\end{array}$ & $\begin{array}{l}4.9 \\
2.9 \\
2.8 \\
3.1 \\
2.6\end{array}$ & $\begin{array}{l}2.2 \\
1.4 \\
1.2 \\
1.1 \\
1.0\end{array}$ & $\begin{array}{r}3.3 \\
3.0 \\
4.6 \\
16 \\
7.6\end{array}$ & $\begin{array}{l}3.0 \\
3.5 \\
3.2 \\
3.0 \\
2.3\end{array}$ & $\begin{array}{l}4.1 \\
3.7 \\
3.6 \\
3.1 \\
3.0\end{array}$ & $\begin{array}{l}7.7 \\
7.2 \\
6.7 \\
6.4 \\
6.2\end{array}$ \\
\hline $\begin{array}{l}16 \\
17 \\
18 \\
19 \\
20\end{array}$ & $\begin{array}{l}.26 \\
.16 \\
.02 \\
0\end{array}$ & $\begin{array}{l}0 \\
0 \\
0 \\
0 \\
0\end{array}$ & $\begin{array}{l}0 \\
0 \\
0 \\
0 \\
0\end{array}$ & $\begin{array}{l}0 \\
.10 \\
.25 \\
1.0 \\
1.5\end{array}$ & $\begin{array}{l}2.2 \\
1.7 \\
1.9 \\
1.8 \\
1.9\end{array}$ & $\begin{array}{r}22 \\
12 \\
8.0 \\
5.0 \\
5.6\end{array}$ & $\begin{array}{l}2.4 \\
1.6 \\
1.6 \\
1.5 \\
2.2\end{array}$ & $\begin{array}{r}1.4 \\
1.5 \\
1.3 \\
3.6 \\
27\end{array}$ & $\begin{array}{l}18 \\
26 \\
31 \\
21 \\
19\end{array}$ & $\begin{array}{c}2.4 \\
2.7 \\
7.1 \\
19 \\
31\end{array}$ & $\begin{array}{l}3.2 \\
4.2 \\
4.6 \\
11 \\
24\end{array}$ & $\begin{array}{l}5.7 \\
5.1 \\
5.0 \\
5.9 \\
5.6\end{array}$ \\
\hline $\begin{array}{l}21 \\
22 \\
23 \\
24 \\
25\end{array}$ & $\begin{array}{l}0 \\
0 \\
0 \\
0 \\
0\end{array}$ & $\begin{array}{l}0 \\
0 \\
0 \\
0 \\
0\end{array}$ & $\begin{array}{l}0 \\
0 \\
0 \\
0 \\
0\end{array}$ & $\begin{array}{l}2.0 \\
1.0 \\
.65 \\
.40 \\
.25\end{array}$ & $\begin{array}{l}2.1 \\
2.2 \\
2.2 \\
1.9 \\
1.6\end{array}$ & $\begin{array}{l}5.5 \\
5.5 \\
4.7 \\
3.9 \\
4.1\end{array}$ & $\begin{array}{l}2.0 \\
1.6 \\
1.1 \\
1.0 \\
.80\end{array}$ & $\begin{array}{c}15 \\
9.3 \\
7.1 \\
5.9 \\
5.8\end{array}$ & $\begin{array}{l}16 \\
35 \\
56 \\
33 \\
23\end{array}$ & $\begin{array}{r}17 \\
9.3 \\
7.1 \\
6.0 \\
5.9\end{array}$ & $\begin{array}{c}17 \\
11 \\
8.6 \\
9.5 \\
426\end{array}$ & $\begin{array}{l}5.0 \\
4.7 \\
4.4 \\
4.2 \\
4.0\end{array}$ \\
\hline $\begin{array}{l}26 \\
27 \\
28 \\
29 \\
30 \\
31\end{array}$ & $\begin{array}{l}0 \\
0 \\
0 \\
.03 \\
.17 \\
.31\end{array}$ & $\begin{array}{l}0 \\
0_{0}^{.01} \\
0 \\
0\end{array}$ & $\begin{array}{l}0 \\
0 \\
0 \\
0 \\
0 \\
0\end{array}$ & $\begin{array}{l}.15 \\
.09 \\
.07 \\
.06 \\
.06 \\
.05\end{array}$ & $\begin{array}{l}1.4 \\
1.7 \\
1.7\end{array}$ & $\begin{array}{l}3.1 \\
2.8 \\
2.8 \\
2.5 \\
2.5 \\
2.5\end{array}$ & $\begin{array}{c}1.0 \\
.74 \\
.83 \\
.96 \\
.76\end{array}$ & $\begin{array}{l}5.5 \\
4.9 \\
5.1 \\
5.2 \\
3.6 \\
3.1\end{array}$ & $\begin{array}{l}25 \\
22 \\
17 \\
15 \\
14\end{array}$ & $\begin{array}{c}5.8 \\
8.9 \\
24 \\
15 \\
10 \\
7.9\end{array}$ & $\begin{array}{r}117 \\
64 \\
44 \\
33 \\
27 \\
23\end{array}$ & $\begin{array}{l}4.0 \\
4.1 \\
3.7 \\
3.7 \\
4.0\end{array}$ \\
\hline \multicolumn{13}{|c|}{ Daily mean specific conductance, in microsiemens per centimeter at 25 degrees Celsius } \\
\hline $\begin{array}{l}1 \\
2 \\
3 \\
4 \\
5\end{array}$ & $\begin{array}{l}--. \\
629 \\
629 \\
618\end{array}$ & $\begin{array}{l}602 \\
-\therefore \\
-- \\
--\end{array}$ & $\begin{array}{l}=- \\
=- \\
=- \\
\cdots\end{array}$ & $\begin{array}{l}\cdots \\
\cdots \\
\cdots \\
\cdots \\
\cdots\end{array}$ & $\begin{array}{l}a- \\
=- \\
\square- \\
\cdots\end{array}$ & $\begin{array}{l}-. \\
\cdots \\
\cdots \\
\cdots\end{array}$ & $\begin{array}{l}a \\
\cdots \\
\cdots \\
-\infty\end{array}$ & $\begin{array}{l}620 \\
623 \\
644 \\
638 \\
645\end{array}$ & $\begin{array}{l}\cdots \\
\cdots \\
\cdots\end{array}$ & $\begin{array}{l}753 \\
733 \\
744 \\
746 \\
730\end{array}$ & $\begin{array}{l}694 \\
703 \\
714 \\
704 \\
674\end{array}$ & $\begin{array}{l}785 \\
773 \\
755 \\
738 \\
727\end{array}$ \\
\hline $\begin{array}{r}6 \\
7 \\
8 \\
9 \\
10\end{array}$ & $\begin{array}{l}613 \\
610 \\
609 \\
610 \\
615\end{array}$ & $\begin{array}{l}\cdots \\
\cdots \\
\cdots- \\
\cdots- \\
\cdots-\end{array}$ & $\begin{array}{l}\cdots \\
\cdots \\
\cdots \\
\cdots\end{array}$ & $\begin{array}{c}-- \\
=- \\
\square- \\
--- \\
--\end{array}$ & \begin{tabular}{l}
--- \\
\hdashline- \\
\hdashline- \\
-- \\
--
\end{tabular} & $\begin{array}{l}-. . \\
-:- \\
\cdots-. \\
-\cdots\end{array}$ & $\begin{array}{l}\cdots \\
\cdots \\
\cdots \\
\cdots \\
\cdots\end{array}$ & $\begin{array}{l}636 \\
651 \\
668 \\
632 \\
594\end{array}$ & \begin{tabular}{l}
$\cdots$ \\
\hdashline- \\
- \\
06
\end{tabular} & $\begin{array}{l}723 \\
707 \\
701 \\
695 \\
689\end{array}$ & $\begin{array}{l}--- \\
-- \\
675 \\
666\end{array}$ & $\begin{array}{l}717 \\
718 \\
721 \\
700 \\
683\end{array}$ \\
\hline $\begin{array}{l}11 \\
12 \\
13 \\
14 \\
15\end{array}$ & $\begin{array}{l}616 \\
616 \\
619 \\
623 \\
619\end{array}$ & $\begin{array}{c}\cdots \\
\cdots \\
\cdots \\
\cdots- \\
\cdots\end{array}$ & $\begin{array}{l}=- \\
\because-- \\
\square-- \\
--\end{array}$ & $\begin{array}{l}\cdots \\
\because- \\
-\cdots \\
--\end{array}$ & $\begin{array}{l}-. \\
\because- \\
\cdots \\
\cdots\end{array}$ & \begin{tabular}{l}
..- \\
\hdashline.- \\
\hdashline.- \\
-
\end{tabular} & $\begin{array}{l}-707 \\
605 \\
597 \\
599\end{array}$ & $\begin{array}{l}582 \\
584 \\
600 \\
631 \\
645\end{array}$ & $\begin{array}{l}695 \\
687 \\
667 \\
633 \\
587\end{array}$ & $\begin{array}{l}694 \\
699 \\
650 \\
640 \\
631\end{array}$ & $\begin{array}{l}682 \\
677 \\
669 \\
674 \\
685\end{array}$ & $\begin{array}{l}-\ldots \\
-382 \\
698 \\
676\end{array}$ \\
\hline $\begin{array}{l}16 \\
17 \\
18 \\
19 \\
20\end{array}$ & \begin{tabular}{l}
589 \\
591 \\
598 \\
\hdashline- \\
-
\end{tabular} & \begin{tabular}{c}
--- \\
$=-$ \\
\hdashline-- \\
---
\end{tabular} & $\begin{array}{l}=- \\
=- \\
\square- \\
-\cdots\end{array}$ & \begin{tabular}{l}
.- \\
$\because-$ \\
\hdashline- \\
--
\end{tabular} & $\begin{array}{l}\ldots \\
\cdots \\
\cdots \\
\cdots \\
\cdots-\end{array}$ & $\begin{array}{l}\cdots \\
\cdots \\
\cdots- \\
\cdots- \\
--\end{array}$ & \begin{tabular}{l}
625 \\
610 \\
-- \\
\hdashline- \\
--
\end{tabular} & $\begin{array}{l}\cdots \\
\cdots \\
\cdots \\
\cdots \\
\cdots-\end{array}$ & $\begin{array}{l}530 \\
572 \\
657 \\
672 \\
698\end{array}$ & $\begin{array}{l}648 \\
631 \\
577 \\
547 \\
512\end{array}$ & $\begin{array}{l}682 \\
650 \\
629 \\
619 \\
612\end{array}$ & $\begin{array}{l}561 \\
630 \\
694 \\
690 \\
690\end{array}$ \\
\hline $\begin{array}{l}21 \\
22 \\
23 \\
24 \\
25\end{array}$ & $\begin{array}{c}\ldots- \\
\cdots \\
\cdots- \\
\cdots- \\
-\cdots\end{array}$ & \begin{tabular}{c}
-- \\
\hdashline- \\
\hdashline- \\
-- \\
-
\end{tabular} & $\begin{array}{l}=- \\
=- \\
=- \\
--\end{array}$ & $\begin{array}{l}--- \\
-\because- \\
--- \\
-\cdots\end{array}$ & $\begin{array}{l}\cdots \\
\cdots \\
\cdots \\
\cdots \\
\cdots\end{array}$ & $\begin{array}{l}740 \\
742 \\
739 \\
731 \\
733\end{array}$ & \begin{tabular}{l}
..- \\
\hdashline$-:$ \\
-- \\
596
\end{tabular} & $\begin{array}{l}\ldots \\
\cdots \\
\cdots \\
\cdots\end{array}$ & $\begin{array}{l}734 \\
619 \\
612 \\
664 \\
768\end{array}$ & $\begin{array}{l}598 \\
616 \\
653 \\
655 \\
-\end{array}$ & $\begin{array}{l}601 \\
636 \\
683 \\
721 \\
530\end{array}$ & $\begin{array}{l}680 \\
681 \\
681 \\
686 \\
684\end{array}$ \\
\hline $\begin{array}{l}26 \\
27 \\
28 \\
29 \\
30 \\
31\end{array}$ & \begin{tabular}{l}
$\cdots-$ \\
$\cdots-$ \\
\hdashline- \\
612 \\
632
\end{tabular} & \begin{tabular}{c}
--- \\
-- \\
\hdashline- \\
-- \\
--- \\
--
\end{tabular} & $\begin{array}{c}--- \\
--- \\
--- \\
-- \\
--\end{array}$ & \begin{tabular}{l}
--- \\
-- \\
\hdashline- \\
$\square-$ \\
-- \\
---
\end{tabular} & $\begin{array}{l}\cdots \\
=- \\
\cdots- \\
\cdots \\
\cdots-\end{array}$ & \begin{tabular}{l}
740 \\
745 \\
744 \\
$-\square$ \\
\hdashline- \\
--
\end{tabular} & $\begin{array}{l}590 \\
591 \\
590 \\
599 \\
608 \\
-\end{array}$ & \begin{tabular}{l}
$=-$ \\
$\because-:$ \\
\hdashline$-:$ \\
\hdashline$-:$ \\
\hdashline-
\end{tabular} & $\begin{array}{l}758 \\
747 \\
728 \\
746 \\
757 \\
--\end{array}$ & $\begin{array}{l}668 \\
652 \\
623 \\
641 \\
650 \\
684\end{array}$ & $\begin{array}{l}600 \\
733 \\
793 \\
797 \\
795 \\
796\end{array}$ & $\begin{array}{l}670 \\
663 \\
626 \\
630 \\
--- \\
---\end{array}$ \\
\hline
\end{tabular}


Table 17. Daily median pH and mean water temperature at site RC2, Roberts Creek above Saint Olaf, Iowa, water year 1990

[---, data not available to calculate mean or median values; water-quality instrumentation was removed from November 27 to March 20]

\begin{tabular}{|c|c|c|c|c|c|c|c|c|c|c|c|c|}
\hline Day & oct. & Nov. & Dec. & Jan. & Feb. & Mar. & Apr. & May & June & July & Aug. & Sept \\
\hline \multicolumn{13}{|c|}{ Daily median $\mathrm{pH}$} \\
\hline $\begin{array}{l}1 \\
2 \\
3 \\
4 \\
5\end{array}$ & $\begin{array}{l}7.9 \\
7.9 \\
6.7 \\
6.6\end{array}$ & \begin{tabular}{c}
7.0 \\
\hdashline- \\
\hdashline- \\
\hdashline
\end{tabular} & $\begin{array}{l}=- \\
=- \\
=- \\
=-\end{array}$ & $\begin{array}{l}--- \\
=-- \\
--- \\
---\end{array}$ & $\begin{array}{l}=- \\
=-- \\
=-- \\
=-\end{array}$ & $\begin{array}{l}--- \\
-- \\
--- \\
---\end{array}$ & $\begin{array}{l}-- \\
-- \\
-- \\
--\end{array}$ & $\begin{array}{l}8.3 \\
8.2 \\
7.9 \\
7.8 \\
7.6\end{array}$ & $\begin{array}{l}--- \\
-\square \\
--- \\
---\end{array}$ & $\begin{array}{l}8.0 \\
8.0 \\
8.2 \\
8.2 \\
8.1\end{array}$ & $\begin{array}{l}8.1 \\
8.2 \\
8.3 \\
8.2 \\
8.2\end{array}$ & $\begin{array}{l}8.2 \\
8.2 \\
8.1 \\
8.0 \\
7.8\end{array}$ \\
\hline $\begin{array}{r}6 \\
7 \\
8 \\
9 \\
10\end{array}$ & $\begin{array}{l}6.9 \\
6.8 \\
6.8 \\
6.7 \\
6.9\end{array}$ & 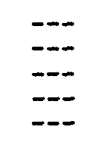 & $\begin{array}{l}=- \\
=- \\
=- \\
=-\end{array}$ & $\begin{array}{l}--- \\
=- \\
--- \\
---\end{array}$ & $\begin{array}{l}=- \\
\overline{--} \\
\overline{-\square} \\
=-\end{array}$ & $\begin{array}{l}=- \\
=- \\
=- \\
--\end{array}$ & $\begin{array}{l}=- \\
=- \\
=- \\
=-\end{array}$ & $\begin{array}{l}8.0 \\
7.9 \\
7.8 \\
7.8 \\
7.9\end{array}$ & 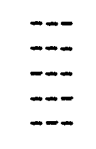 & $\begin{array}{l}8.2 \\
8.3 \\
8.2 \\
8.3 \\
8.3\end{array}$ & \begin{tabular}{c}
$\cdots$ \\
$\cdots$ \\
\hdashline .1 \\
8.0
\end{tabular} & $\begin{array}{l}8.0 \\
8.3 \\
8.1 \\
8.0 \\
7.9\end{array}$ \\
\hline $\begin{array}{l}11 \\
12 \\
13 \\
14 \\
15\end{array}$ & $\begin{array}{l}7.1 \\
7.2 \\
7.2 \\
7.1 \\
7.0\end{array}$ & 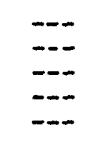 & $\begin{array}{l}=- \\
=- \\
=-- \\
---\end{array}$ & $\begin{array}{l}=- \\
=- \\
a- \\
\square-\end{array}$ & $\begin{array}{l}--- \\
=-- \\
=-- \\
---\end{array}$ & $\begin{array}{l}=- \\
=- \\
-- \\
--\end{array}$ & $\begin{array}{l}.--- \\
9.1 \\
9.1 \\
9.1 \\
8.9\end{array}$ & $\begin{array}{l}7.9 \\
7.7 \\
7.8 \\
7.8 \\
-9\end{array}$ & $\begin{array}{l}8.2 \\
8.2 \\
8.0 \\
7.9 \\
7.7\end{array}$ & $\begin{array}{l}8.4 \\
8.3 \\
8.4 \\
8.3 \\
8.4\end{array}$ & $\begin{array}{l}8.1 \\
8.0 \\
8.0 \\
8.1 \\
8.0\end{array}$ & $\begin{array}{c}--- \\
7.6 \\
7.7 \\
7.8\end{array}$ \\
\hline $\begin{array}{l}16 \\
17 \\
18 \\
19 \\
20\end{array}$ & $\begin{array}{r}7.0 \\
5.8 \\
6.8 \\
--. \\
-\cdots\end{array}$ & \begin{tabular}{l}
$=-$ \\
\hdashline- \\
$=-$ \\
-- \\
-
\end{tabular} & $\begin{array}{l}=- \\
\overline{--} \\
--- \\
---\end{array}$ & $\begin{array}{l}--- \\
--- \\
--- \\
---\end{array}$ & $\begin{array}{l}--- \\
=- \\
=- \\
=-\end{array}$ & $\begin{array}{l}\square- \\
-- \\
-- \\
-\cdots\end{array}$ & \begin{tabular}{l}
$\mathbf{9 . 0}$ \\
$\mathbf{9 . 1}$ \\
\hdashline.- \\
\hdashline-
\end{tabular} & $\begin{array}{l}=- \\
=-- \\
-- \\
---\end{array}$ & $\begin{array}{l}7.5 \\
7.6 \\
7.5 \\
7.5 \\
7.6\end{array}$ & $\begin{array}{l}8.4 \\
8.3 \\
7.9 \\
7.8 \\
7.6\end{array}$ & $\begin{array}{l}8.1 \\
8.0 \\
8.1 \\
8.1 \\
8.0\end{array}$ & $\begin{array}{l}7.9 \\
8.3 \\
8.3 \\
8.3 \\
8.3\end{array}$ \\
\hline $\begin{array}{l}21 \\
22 \\
23 \\
24 \\
25\end{array}$ & 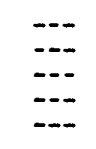 & 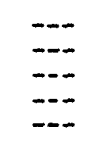 & $\begin{array}{l}--- \\
-\square \\
--- \\
---\end{array}$ & $\begin{array}{l}--- \\
--- \\
--- \\
---\end{array}$ & $\begin{array}{l}=- \\
=-- \\
=-- \\
=--\end{array}$ & $\begin{array}{l}--.- \\
-8.6 \\
8.4 \\
8.4\end{array}$ & $\begin{array}{c}=- \\
=- \\
8.3\end{array}$ & 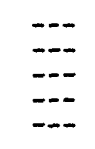 & $\begin{array}{l}7.6 \\
7.5 \\
7.3 \\
7.5 \\
7.9\end{array}$ & $\begin{array}{l}7.6 \\
7.8 \\
8.1 \\
8.3 \\
8.2\end{array}$ & $\begin{array}{l}7.9 \\
8.0 \\
7.9 \\
8.0 \\
7.2\end{array}$ & $\begin{array}{l}8.3 \\
8.2 \\
8.1 \\
8.1 \\
8.0\end{array}$ \\
\hline $\begin{array}{l}26 \\
27 \\
28 \\
29 \\
30 \\
31\end{array}$ & \begin{tabular}{c}
$=-$ \\
\hdashline- \\
$7 \because-1$ \\
7.0
\end{tabular} & \begin{tabular}{l}
-- \\
\hdashline- \\
\hdashline- \\
--
\end{tabular} & $\begin{array}{l}=- \\
=--\end{array}$ & \begin{tabular}{l}
$a--$ \\
$=--$ \\
\hdashline-- \\
$-\cdots$ \\
$-\cdots$
\end{tabular} & $\begin{array}{l}=-- \\
=-- \\
=-- \\
=-- \\
=-\end{array}$ & \begin{tabular}{l}
8.4 \\
8.4 \\
8.4 \\
\hdashline- \\
--
\end{tabular} & $\begin{array}{l}8.8 \\
8.5 \\
8.3 \\
8.5 \\
8.6\end{array}$ & 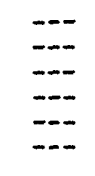 & $\begin{array}{l}7.9 \\
7.9 \\
7.8 \\
7.8 \\
8.0\end{array}$ & $\begin{array}{l}8.1 \\
8.1 \\
8.0 \\
7.8 \\
8.3 \\
8.1\end{array}$ & $\begin{array}{l}7.6 \\
7.8 \\
8.0 \\
8.0 \\
8.1 \\
8.2\end{array}$ & \begin{tabular}{c}
8.0 \\
\hdashline- \\
\hdashline- \\
--
\end{tabular} \\
\hline \multicolumn{13}{|c|}{ Daily mean water temperature, in degrees Celsius } \\
\hline $\begin{array}{l}1 \\
2 \\
3 \\
4 \\
5\end{array}$ & $\begin{array}{r}-.- \\
10.0 \\
9.0 \\
10.5\end{array}$ & \begin{tabular}{l}
7.5 \\
\hdashline- \\
- \\
\hdashline-
\end{tabular} & $\begin{array}{l}=- \\
=- \\
=- \\
=-\end{array}$ & $\begin{array}{l}--- \\
-- \\
--- \\
---\end{array}$ & $\begin{array}{c}=- \\
=- \\
\square=- \\
-\square\end{array}$ & \begin{tabular}{l}
-- \\
\hdashline- \\
--
\end{tabular} & \begin{tabular}{l}
$=-$ \\
\hdashline- \\
\hdashline- \\
--
\end{tabular} & $\begin{array}{l}14.5 \\
15.5 \\
15.0 \\
12.5 \\
14.5\end{array}$ & $\begin{array}{l}-- \\
\because- \\
\because- \\
\cdots-\end{array}$ & $\begin{array}{l}26.0 \\
26.0 \\
26.0 \\
27.5 \\
26.5\end{array}$ & $\begin{array}{l}21.0 \\
21.5 \\
23.0 \\
24.0 \\
22.0\end{array}$ & $\begin{array}{l}21.5 \\
21.0 \\
20.5 \\
22.0 \\
24.0\end{array}$ \\
\hline $\begin{array}{r}6 \\
7 \\
8 \\
9 \\
10\end{array}$ & $\begin{array}{r}10.5 \\
9.0 \\
8.5 \\
8.5 \\
10.0\end{array}$ & $\begin{array}{l}-.- \\
-:- \\
-:- \\
-\cdots\end{array}$ & $\begin{array}{l}=- \\
=- \\
=- \\
=-\end{array}$ & $\begin{array}{l}--- \\
--- \\
--- \\
---\end{array}$ & \begin{tabular}{l}
$=-$ \\
$=-$ \\
\hdashline-- \\
$-\cdots$
\end{tabular} & $\begin{array}{l}-- \\
-- \\
-- \\
--\end{array}$ & $\begin{array}{l}-- \\
\square- \\
\square- \\
-- \\
--\end{array}$ & $\begin{array}{l}17.0 \\
19.0 \\
20.0 \\
15.5 \\
11.5\end{array}$ & $\begin{array}{c}=- \\
\because=- \\
21.0\end{array}$ & $\begin{array}{l}25.5 \\
25.5 \\
25.0 \\
25.0 \\
25.0\end{array}$ & \begin{tabular}{r}
$\cdots-$. \\
\hdashline..- \\
21.0 \\
22.5
\end{tabular} & $\begin{array}{l}25.0 \\
23.5 \\
21.5 \\
20.0 \\
20.5\end{array}$ \\
\hline $\begin{array}{l}11 \\
12 \\
13 \\
14 \\
15\end{array}$ & $\begin{array}{l}11.0 \\
12.5 \\
12.0 \\
14.0 \\
15.5\end{array}$ & 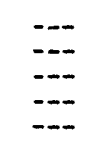 & $\begin{array}{l}=- \\
=- \\
=- \\
=-\end{array}$ & $\begin{array}{l}\square-- \\
=- \\
\square=- \\
---\end{array}$ & $\begin{array}{l}=- \\
=- \\
=- \\
=-\end{array}$ & \begin{tabular}{l}
-- \\
-- \\
\hdashline- \\
--
\end{tabular} & $\begin{array}{r}--- \\
6.0 \\
9.5 \\
12.0 \\
8.5\end{array}$ & $\begin{array}{l}14.0 \\
14.0 \\
15.5 \\
16.0 \\
15.0\end{array}$ & $\begin{array}{l}21.5 \\
21.0 \\
22.5 \\
23.5 \\
22.5\end{array}$ & $\begin{array}{l}25.5 \\
25.0 \\
25.0 \\
25.0 \\
25.5\end{array}$ & $\begin{array}{l}21.0 \\
21.0 \\
22.5 \\
21.5 \\
22.0\end{array}$ & $\begin{array}{r}-.- \\
19.5 \\
21.0 \\
19.5\end{array}$ \\
\hline $\begin{array}{l}16 \\
17 \\
18 \\
19 \\
20\end{array}$ & $\begin{array}{r}13.5 \\
9.0 \\
7.5 \\
-- \\
--\end{array}$ & \begin{tabular}{l}
--- \\
\hdashline$-\overline{-}$ \\
\hdashline- \\
$-\cdots$
\end{tabular} & $\begin{array}{c}=- \\
=- \\
\overline{-} \\
\overline{-}\end{array}$ & $\begin{array}{l}--- \\
--- \\
--- \\
---\end{array}$ & $\begin{array}{l}=-- \\
=-- \\
=-- \\
-\cdots\end{array}$ & $\begin{array}{l}-- \\
=- \\
--- \\
--\end{array}$ & $\begin{array}{l}8.0 \\
9.5 \\
- \\
- \\
-\end{array}$ & $\begin{array}{l}=- \\
=- \\
\square= \\
\square\end{array}$ & $\begin{array}{l}21.5 \\
21.5 \\
23.0 \\
22.5 \\
22.5\end{array}$ & $\begin{array}{l}25.5 \\
25.5 \\
23.0 \\
21.5 \\
21.5\end{array}$ & $\begin{array}{l}23.5 \\
23.0 \\
25.5 \\
23.5 \\
21.0\end{array}$ & $\begin{array}{l}13.5 \\
14.5 \\
14.5 \\
15.5 \\
15.5\end{array}$ \\
\hline $\begin{array}{l}21 \\
22 \\
23 \\
24 \\
25\end{array}$ & \begin{tabular}{l}
--- \\
$\cdots-$ \\
\hdashline- \\
-- \\
-
\end{tabular} & \begin{tabular}{l}
--- \\
-- \\
\hdashline- \\
--
\end{tabular} & $\begin{array}{l}\cdots \\
\square \\
\cdots \\
\cdots\end{array}$ & \begin{tabular}{l}
--- \\
\hdashline$-:$ \\
$-\because-$ \\
$-\cdots$
\end{tabular} & $\begin{array}{l}-\cdots \\
\because-- \\
-\because- \\
-\cdots\end{array}$ & $\begin{array}{r}10.0 \\
7.5 \\
2.0 \\
3.0 \\
3.5\end{array}$ & $\begin{array}{c}=- \\
\because- \\
\because- \\
--\end{array}$ & \begin{tabular}{l}
$=-$ \\
\hdashline- \\
$\cdots$ \\
$\cdots$
\end{tabular} & $\begin{array}{l}22.0 \\
20.0 \\
19.0 \\
21.5 \\
23.5\end{array}$ & $\begin{array}{l}21.0 \\
21.5 \\
21.5 \\
21.5 \\
-.-\end{array}$ & $\begin{array}{l}19.5 \\
21.0 \\
21.5 \\
23.0 \\
22.0\end{array}$ & $\begin{array}{r}17.5 \\
16.0 \\
-\therefore \\
\hdashline-\end{array}$ \\
\hline $\begin{array}{l}26 \\
27 \\
28 \\
29 \\
30 \\
31\end{array}$ & \begin{tabular}{r}
..- \\
\hdashline$\overline{12.5}$ \\
9.0
\end{tabular} & \begin{tabular}{l}
.-- \\
$-:-$ \\
\hdashline- \\
--
\end{tabular} & $\begin{array}{l}--- \\
=-- \\
=-- \\
-\square \\
---\end{array}$ & $\begin{array}{c}--- \\
=-- \\
=-- \\
-a \\
-\square\end{array}$ & $\begin{array}{l}--- \\
-\cdots\end{array}$ & $\begin{array}{l}4.5 \\
5.5 \\
5.0 \\
-- \\
-.\end{array}$ & $\begin{array}{l}21.5 \\
19.5 \\
16.0 \\
15.5 \\
14.5\end{array}$ & $\begin{array}{l}18.5 \\
17.5 \\
17.5 \\
17.0 \\
16.5\end{array}$ & $\begin{array}{l}24.0 \\
24.5 \\
23.5 \\
23.0 \\
25.0\end{array}$ & $\begin{array}{l}21.5 \\
20.5 \\
22.0 \\
23.5 \\
23.0 \\
22.0\end{array}$ & $\begin{array}{l}21.0 \\
22.5 \\
24.0 \\
23.0 \\
21.5 \\
21.0\end{array}$ & $\begin{array}{l}16.5 \\
16.0 \\
18.0 \\
16.0\end{array}$ \\
\hline
\end{tabular}


Table 18. Daily mean suspended-sediment concentrations and daily suspended-sediment load at site RC2, Roberts Creek above Saint Olaf, Iowa, water year 1990

[--, data not available to calculate values]

Day Oct. Nov. Dec. Jan. Feb. Mar. Apr. May June Jugt.

Daily mean suspended-sediment concentration, in milligrams per liter

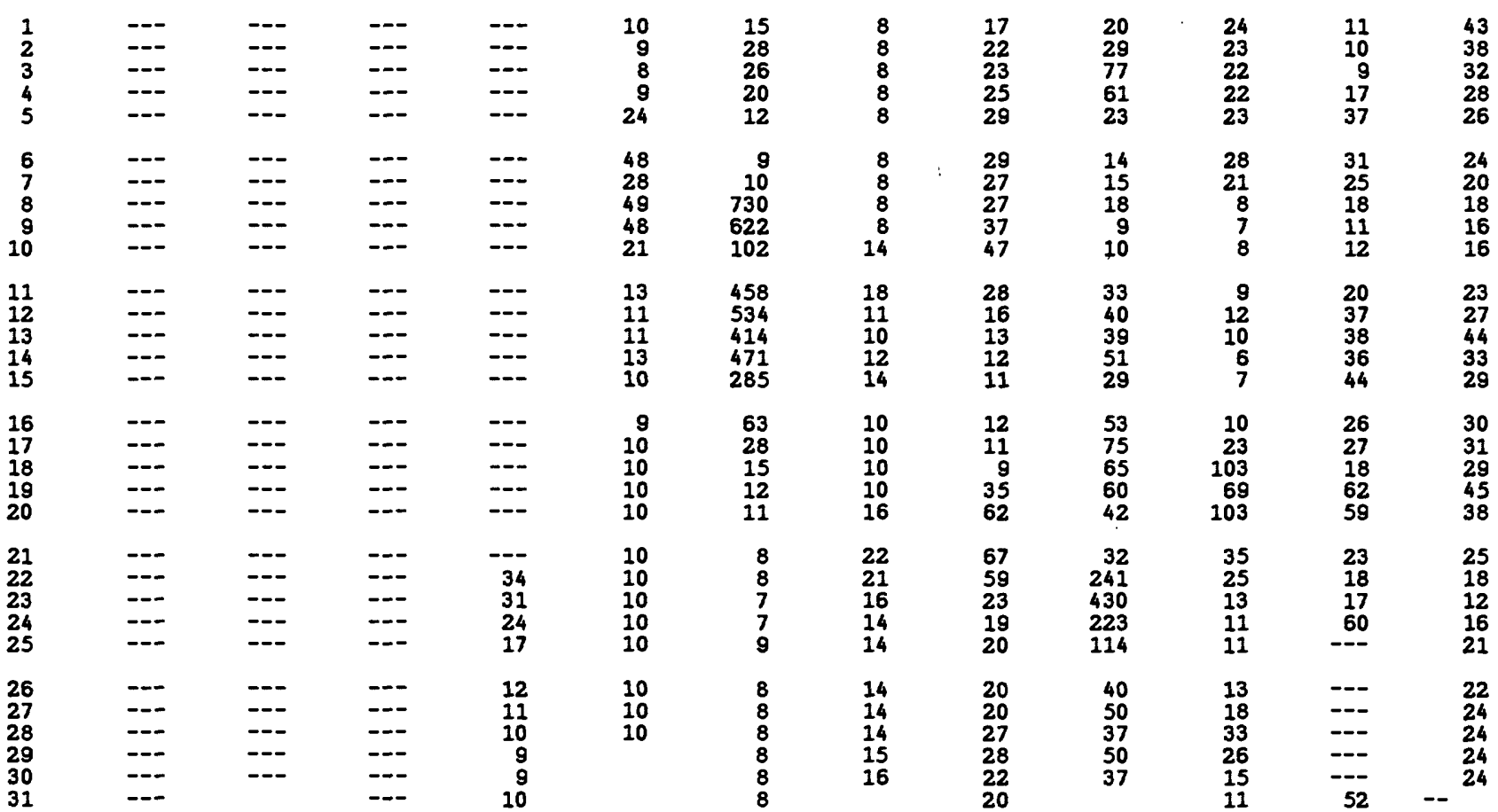

Daily suspended-sediment load, in tons

\begin{tabular}{|c|c|c|c|c|c|c|c|c|c|c|c|c|}
\hline $\begin{array}{l}1 \\
2 \\
3 \\
4 \\
5\end{array}$ & $\begin{array}{l}--- \\
--- \\
--- \\
--- \\
---\end{array}$ & $\begin{array}{l}0^{---} \\
0 \\
0 \\
0\end{array}$ & $\begin{array}{l}0 \\
0 \\
0 \\
0 \\
0\end{array}$ & $\begin{array}{l}0 \\
0 \\
0 \\
0 \\
0\end{array}$ & $\begin{array}{l}0.08 \\
.08 \\
.07 \\
.07 \\
2.7\end{array}$ & $\begin{array}{r}0.16 \\
.70 \\
.90 \\
.72 \\
.23\end{array}$ & $\begin{array}{l}0.06 \\
.05 \\
.05 \\
.06 \\
.06\end{array}$ & $\begin{array}{l}0.04 \\
.04 \\
.04 \\
.05 \\
.06\end{array}$ & $\begin{array}{l}0.15 \\
.72 \\
3.4 \\
1.4 \\
.42\end{array}$ & $\begin{array}{r}0.69 \\
.54 \\
.46 \\
.46 \\
.39\end{array}$ & $\begin{array}{r}0.19 \\
.17 \\
.14 \\
.24 \\
1.9\end{array}$ & $\begin{array}{l}2.4 \\
1.8 \\
1.4 \\
1.1 \\
1.1\end{array}$ \\
\hline $\begin{array}{r}6 \\
7 \\
8 \\
9 \\
10\end{array}$ & $\begin{array}{l}--- \\
--- \\
--- \\
--- \\
---\end{array}$ & $\begin{array}{l}0 \\
0 \\
0 \\
0 \\
0\end{array}$ & $\begin{array}{l}0 \\
0 \\
0 \\
0 \\
0\end{array}$ & $\begin{array}{l}0 \\
0 \\
0 \\
0 \\
0\end{array}$ & $\begin{array}{l}16 \\
3.8 \\
21 \\
19 \\
1.7\end{array}$ & $2,270^{.08} 54$ & $\begin{array}{l}.05 \\
.04 \\
.04 \\
.04 \\
.12\end{array}$ & $\begin{array}{l}.07 \\
.06 \\
.05 \\
.11 \\
.32\end{array}$ & $\begin{array}{l}.25 \\
.24 \\
.24 \\
.11 \\
.09\end{array}$ & $\begin{array}{l}.41 \\
.25 \\
.12 \\
.08 \\
.07\end{array}$ & $\begin{array}{l}.67 \\
.41 \\
.26 \\
.15 \\
.14\end{array}$ & $\begin{array}{l}.85 \\
.62 \\
.49 \\
.40 \\
.37\end{array}$ \\
\hline $\begin{array}{l}11 \\
12 \\
13 \\
14 \\
15\end{array}$ & $\begin{array}{l}--- \\
--- \\
--- \\
--- \\
--\infty\end{array}$ & $\begin{array}{l}0 \\
0 \\
0 \\
0 \\
0\end{array}$ & $\begin{array}{l}0 \\
0 \\
0 \\
0 \\
0\end{array}$ & $\begin{array}{l}0 \\
0 \\
0 \\
0 \\
0\end{array}$ & $\begin{array}{l}.51 \\
.32 \\
.28 \\
.21 \\
.11\end{array}$ & $\begin{array}{r}290 \\
117 \\
44 \\
129 \\
33\end{array}$ & $\begin{array}{l}.25 \\
.09 \\
.08 \\
.10 \\
.10\end{array}$ & $\begin{array}{l}.17 \\
.06 \\
.04 \\
.04 \\
.03\end{array}$ & $\begin{array}{r}.30 \\
.33 \\
.50 \\
2.2 \\
.60\end{array}$ & $\begin{array}{l}.07 \\
.11 \\
.08 \\
.05 \\
.04\end{array}$ & $\begin{array}{l}.22 \\
.37 \\
.37 \\
.31 \\
.36\end{array}$ & $\begin{array}{l}.47 \\
.53 \\
.80 \\
.57 \\
.49\end{array}$ \\
\hline $\begin{array}{l}16 \\
17 \\
18 \\
19 \\
20\end{array}$ & $\begin{array}{l}--- \\
\overline{---} \\
0\end{array}$ & $\begin{array}{l}0 \\
0 \\
0 \\
0 \\
0\end{array}$ & $\begin{array}{l}0 \\
0 \\
0 \\
0 \\
0\end{array}$ & $\begin{array}{l}0 \\
0 \\
0 \\
0 \\
0\end{array}$ & $\begin{array}{l}.08 \\
.07 \\
.08 \\
.07 \\
.06\end{array}$ & $\begin{array}{l}4.0 \\
.92 \\
.32 \\
.16 \\
.17\end{array}$ & $\begin{array}{l}.06 \\
.04 \\
.04 \\
.04 \\
.10\end{array}$ & $\begin{array}{l}.05 \\
.04 \\
.03 \\
.40 \\
4.7\end{array}$ & $\begin{array}{l}3.3 \\
5.3 \\
5.6 \\
3.4 \\
2.2\end{array}$ & $\begin{array}{l}.06 \\
.17 \\
2.7 \\
3.6 \\
8.9\end{array}$ & $\begin{array}{r}.23 \\
.33 \\
.23 \\
1.9 \\
3.9\end{array}$ & $\begin{array}{l}.46 \\
.43 \\
.40 \\
.73 \\
.57\end{array}$ \\
\hline $\begin{array}{l}21 \\
22 \\
23 \\
24 \\
25\end{array}$ & $\begin{array}{l}0 \\
0 \\
0 \\
0 \\
0\end{array}$ & $\begin{array}{l}0 \\
0 \\
0 \\
0 \\
0\end{array}$ & $\begin{array}{l}0 \\
0 \\
0 \\
0 \\
0\end{array}$ & $\begin{array}{l}0 \\
.64 \\
.54 \\
.35 \\
.22\end{array}$ & $\begin{array}{l}.07 \\
.07 \\
.07 \\
.07 \\
.06\end{array}$ & $\begin{array}{l}.12 \\
.12 \\
.10 \\
.08 \\
.10\end{array}$ & $\begin{array}{l}.12 \\
.09 \\
.05 \\
.04 \\
.03\end{array}$ & $\begin{array}{l}2.7 \\
1.5 \\
.44 \\
.31 \\
.31\end{array}$ & $\begin{array}{l}1.4 \\
30 \\
68 \\
20 \\
7.2\end{array}$ & $\begin{array}{r}1.7 \\
.65 \\
.25 \\
.18 \\
.17\end{array}$ & $\begin{array}{r}1.0 \\
.56 \\
.39 \\
2.1 \\
960^{.1}\end{array}$ & $\begin{array}{l}.33 \\
.23 \\
.15 \\
.18 \\
.23\end{array}$ \\
\hline $\begin{array}{l}26 \\
27 \\
28 \\
29 \\
30 \\
31\end{array}$ & $\begin{array}{l}0 \\
0 \\
0 \\
-\ldots- \\
---\end{array}$ & $\begin{array}{l}0 \\
0 \\
0 \\
0 \\
0\end{array}$ & $\begin{array}{l}0 \\
0 \\
0 \\
0 \\
0 \\
0\end{array}$ & $\begin{array}{l}.14 \\
.11 \\
.09 \\
.08 \\
.08 \\
.08\end{array}$ & $\begin{array}{l}.06 \\
.06 \\
.06\end{array}$ & $\begin{array}{l}.07 \\
.06 \\
.06 \\
.05 \\
.05 \\
.05\end{array}$ & $\begin{array}{l}.04 \\
.03 \\
.03 \\
.04 \\
.03\end{array}$ & $\begin{array}{l}.30 \\
.27 \\
.38 \\
.39 \\
.22 \\
.17\end{array}$ & $\begin{array}{l}2.9 \\
3.0 \\
1.7 \\
2.1 \\
1.4\end{array}$ & $\begin{array}{l}.21 \\
.51 \\
2.1 \\
1.1 \\
.42 \\
.24\end{array}$ & $\begin{array}{r}57 \\
52 \\
26 \\
21 \\
5.0 \\
3.3\end{array}$ & $\begin{array}{l}.24 \\
.27 \\
.25 \\
.24 \\
.26\end{array}$ \\
\hline
\end{tabular}

Total

measured 0

0

0

$2.33 \quad 66.8 \quad 3,450$

1.97

13.4

168

$26.83,140$

18.4 
Table 19. Miscellaneous water-level measurements in the Big Spring basin, Clayton County, Iowa, water year 1990

[Water levels in feet below land surface; negative value is water level above land surface]

\begin{tabular}{|c|c|c|c|}
\hline Date & Water level & Date & Water level \\
\hline \multicolumn{4}{|c|}{ Well BS1-D } \\
\hline $\begin{array}{r}10-05-89 \\
11-07-89 \\
12-04-89 \\
1-03-90 \\
1-16-90 \\
1-26-90 \\
2-06-90 \\
2-12-90 \\
2-22-90\end{array}$ & $\begin{array}{l}-4.10 \\
-3.87 \\
-3.80 \\
-3.31 \\
-3.64 \\
-3.38 \\
-3.36 \\
-3.38 \\
-3.36\end{array}$ & $\begin{array}{l}3-05-90 \\
3-09-90 \\
5-01-90 \\
5-09-90 \\
5-22-90 \\
6-05-90 \\
5-29-90 \\
7-02-90 \\
8-26-90 \\
9-05-90\end{array}$ & $\begin{array}{l}-3.35 \\
-3.36 \\
-3.71 \\
-3.99 \\
-4.01 \\
-4.22 \\
-4.26 \\
-4.10 \\
-5.03 \\
-4.52\end{array}$ \\
\hline \multicolumn{4}{|c|}{ Well BS2-A } \\
\hline $\begin{array}{l}5-02-90 \\
5-05-90 \\
6-29-90\end{array}$ & $\begin{array}{l}55.22 \\
55.29 \\
55.30\end{array}$ & $\begin{array}{l}8-01-90 \\
9-05-90\end{array}$ & $\begin{array}{l}55.30 \\
55.33\end{array}$ \\
\hline \multicolumn{4}{|c|}{ We1I BS2-B } \\
\hline $\begin{array}{l}5-02-90 \\
6-05-90 \\
6-29-90\end{array}$ & $\begin{array}{l}126.06 \\
126.04 \\
126.10\end{array}$ & $\begin{array}{l}8-01-90 \\
9-05-90\end{array}$ & $\begin{array}{l}126.07 \\
126.05\end{array}$ \\
\hline \multicolumn{4}{|c|}{ Well BS2-C } \\
\hline $\begin{array}{l}5-02-90 \\
6-05-90 \\
6-29-90\end{array}$ & $\begin{array}{l}124.47 \\
124.98 \\
123.70\end{array}$ & $\begin{array}{l}8-01-90 \\
9-05-90\end{array}$ & $\begin{array}{l}123.71 \\
123.40\end{array}$ \\
\hline \multicolumn{4}{|c|}{ Well BS2-D } \\
\hline $\begin{array}{l}5-02-90 \\
5-05-90 \\
6-29-90\end{array}$ & $\begin{array}{l}136.71 \\
135.14 \\
135.02\end{array}$ & $\begin{array}{l}8-01-90 \\
9-05-90\end{array}$ & $\begin{array}{l}134.83 \\
134.69\end{array}$ \\
\hline \multicolumn{4}{|c|}{ Well BS2-F } \\
\hline \multirow[t]{2}{*}{$\begin{array}{l}5-02-90 \\
6-05-90 \\
6-29-90\end{array}$} & $\begin{array}{l}282.44 \\
282.07 \\
281.80\end{array}$ & $\begin{array}{l}8-01-90 \\
9-05-90\end{array}$ & $\begin{array}{l}281.69 \\
281.64\end{array}$ \\
\hline & \multicolumn{3}{|c|}{ Well BS3 } \\
\hline $\begin{array}{r}10-05-89 \\
11-07-89 \\
12-04-89 \\
1-03-90 \\
2-06-90 \\
3-05-90\end{array}$ & $\begin{array}{l}300.04 \\
300.01 \\
299.98 \\
300.28 \\
300.28 \\
300.55\end{array}$ & $\begin{array}{l}4-02-90 \\
5-02-90 \\
6-29-90 \\
8-01-90 \\
8-13-90 \\
9-05-90\end{array}$ & $\begin{array}{l}300.52 \\
300.48 \\
300.54 \\
300.71 \\
300.77 \\
300.65\end{array}$ \\
\hline
\end{tabular}


Table 20. Daily mean water levels in unconsolidated aquifers, Clayton County, Iowa, water year 1990

[Water levels in feet below land surface; --, data not available to calculate mean values]

\begin{tabular}{|c|c|c|c|c|c|c|c|c|c|c|c|c|}
\hline Day & Oct. & Nov. & Dec. & Jan. & Feb. & Mar. & Apr. & May & June & July & Aus. & Sept \\
\hline & & \multicolumn{9}{|c|}{ Well BS1-A (fig. 2) } & \multicolumn{2}{|r|}{ - } \\
\hline $\begin{array}{l}1 \\
2 \\
3 \\
4 \\
5\end{array}$ & $\begin{array}{l}14.61 \\
14.64 \\
14.65 \\
14.66 \\
14.65\end{array}$ & $\begin{array}{l}14.46 \\
14.45 \\
14.45 \\
14.45 \\
14.45\end{array}$ & $\begin{array}{l}14.60 \\
14.58 \\
14.66 \\
14.62 \\
14.61\end{array}$ & $\begin{array}{l}14.70 \\
14.70 \\
14.68 \\
14.66 \\
14.64\end{array}$ & $\begin{array}{l}14.57 \\
14.58 \\
14.59 \\
14.65 \\
14.59\end{array}$ & $\begin{array}{l}14.50 \\
14.39 \\
14.28 \\
14.31 \\
14.35\end{array}$ & $\begin{array}{l}13.78 \\
13.81 \\
13.83 \\
13.85 \\
13.88\end{array}$ & $\begin{array}{l}13.81 \\
13.78 \\
13.79 \\
13.81 \\
13.85\end{array}$ & $\begin{array}{l}13.33 \\
13.38 \\
13.38 \\
13.38 \\
13.36\end{array}$ & $\begin{array}{l}13.02 \\
13.15 \\
13.23 \\
13.33 \\
13.44\end{array}$ & $\begin{array}{l}12.46 \\
12.74 \\
12.97 \\
12.94 \\
13.06\end{array}$ & $\begin{array}{l}--- \\
\overline{--} \\
-\cdots \\
---\end{array}$ \\
\hline $\begin{array}{r}6 \\
7 \\
8 \\
9 \\
10\end{array}$ & $\begin{array}{l}14.59 \\
14.57 \\
14.59 \\
14.60 \\
14.62\end{array}$ & $\begin{array}{l}14.45 \\
14.47 \\
14.50 \\
14.50 \\
14.52\end{array}$ & $\begin{array}{l}14.61 \\
14.70 \\
14.67 \\
14.67 \\
14.67\end{array}$ & $\begin{array}{l}14.60 \\
14.59 \\
14.59 \\
14.56 \\
14.29\end{array}$ & $\begin{array}{l}14.19 \\
14.05 \\
13.94 \\
13.38 \\
13.33\end{array}$ & $\begin{array}{l}14.38 \\
14.30 \\
13.88 \\
12.00 \\
11.26\end{array}$ & $\begin{array}{l}13.92 \\
13.95 \\
13.98 \\
14.00 \\
14.00\end{array}$ & $\begin{array}{l}13.89 \\
13.93 \\
13.98 \\
13.99 \\
13.88\end{array}$ & $\begin{array}{l}13.40 \\
13.44 \\
13.48 \\
13.53 \\
13.60\end{array}$ & $\begin{array}{l}13.52 \\
13.58 \\
13.65 \\
13.70 \\
13.76\end{array}$ & $\begin{array}{l}12.85 \\
12.93 \\
13.13 \\
13.30 \\
13.43\end{array}$ & $\begin{array}{l}11.42 \\
11.57 \\
11.69 \\
11.73 \\
11.82\end{array}$ \\
\hline $\begin{array}{l}11 \\
12 \\
13 \\
14 \\
15\end{array}$ & $\begin{array}{l}14.62 \\
14.63 \\
14.63 \\
14.64 \\
14.64\end{array}$ & $\begin{array}{l}14.53 \\
14.54 \\
14.54 \\
14.55 \\
14.56\end{array}$ & $\begin{array}{l}14.69 \\
14.72 \\
14.71 \\
14.71 \\
14.71\end{array}$ & $\begin{array}{l}14.28 \\
14.41 \\
14.51 \\
14.54 \\
14.55\end{array}$ & $\begin{array}{l}13.64 \\
13.80 \\
13.86 \\
13.98 \\
14.19\end{array}$ & $\begin{array}{l}11.03 \\
10.81 \\
10.66 \\
11.05 \\
10.35\end{array}$ & $\begin{array}{l}13.97 \\
13.96 \\
13.97 \\
13.97 \\
13.98\end{array}$ & $\begin{array}{l}13.74 \\
13.55 \\
13.47 \\
13.47 \\
13.54\end{array}$ & $\begin{array}{l}13.66 \\
13.72 \\
13.54 \\
12.87 \\
12.59\end{array}$ & $\begin{array}{l}13.79 \\
13.81 \\
13.79 \\
13.82 \\
13.86\end{array}$ & $\begin{array}{l}13.50 \\
13.47 \\
13.50 \\
13.55 \\
13.44\end{array}$ & $\begin{array}{l}11.93 \\
12.01 \\
12.10 \\
12.17 \\
12.24\end{array}$ \\
\hline $\begin{array}{l}16 \\
17 \\
18 \\
19 \\
20\end{array}$ & $\begin{array}{l}14.57 \\
14.51 \\
14.53 \\
14.54 \\
14.56\end{array}$ & $\begin{array}{l}14.56 \\
14.60 \\
14.62 \\
14.62 \\
14.62\end{array}$ & $\begin{array}{l}14.71 \\
14.71 \\
14.71 \\
14.71 \\
14.72\end{array}$ & $\begin{array}{l}14.56 \\
14.31 \\
13.98 \\
14.17 \\
14.24\end{array}$ & $\begin{array}{l}14.19 \\
14.20 \\
14.20 \\
14.24 \\
14.29\end{array}$ & $\begin{array}{r}9.97 \\
10.95 \\
11.70 \\
12.19 \\
12.53\end{array}$ & $\begin{array}{l}13.98 \\
13.98 \\
13.99 \\
14.00 \\
13.99\end{array}$ & $\begin{array}{l}13.57 \\
13.62 \\
13.67 \\
13.58 \\
13.06\end{array}$ & $\begin{array}{l}12.53 \\
12.52 \\
12.39 \\
12.23 \\
12.29\end{array}$ & $\begin{array}{l}13.90 \\
13.93 \\
13.97 \\
13.94 \\
13.74\end{array}$ & $\begin{array}{l}13.40 \\
13.42 \\
13.35 \\
13.17 \\
12.63\end{array}$ & $\begin{array}{l}12.32 \\
12.38 \\
12.45 \\
12.49 \\
12.48\end{array}$ \\
\hline $\begin{array}{l}21 \\
22 \\
23 \\
24 \\
25\end{array}$ & $\begin{array}{l}14.57 \\
14.58 \\
14.59 \\
14.59 \\
14.59\end{array}$ & $\begin{array}{l}14.61 \\
14.62 \\
14.62 \\
14.63 \\
14.60\end{array}$ & $\begin{array}{l}14.72 \\
14.73 \\
14.73 \\
14.74 \\
14.74\end{array}$ & $\begin{array}{l}14.32 \\
14.39 \\
14.43 \\
14.46 \\
14.48\end{array}$ & $\begin{array}{l}14.33 \\
14.34 \\
14.38 \\
14.40 \\
14.43\end{array}$ & $\begin{array}{l}12.76 \\
12.95 \\
13.08 \\
13.20 \\
13.32\end{array}$ & $\begin{array}{l}13.94 \\
13.94 \\
13.97 \\
14.00 \\
14.02\end{array}$ & $\begin{array}{l}12.13 \\
12.00 \\
12.20 \\
12.41 \\
12.58\end{array}$ & $\begin{array}{l}12.43 \\
12.29 \\
11.90 \\
11.86 \\
12.10\end{array}$ & $\begin{array}{l}13.52 \\
13.47 \\
13.58 \\
13.67 \\
13.73\end{array}$ & $\begin{array}{r}11.24 \\
11.03 \\
11.48 \\
12.09 \\
7.75\end{array}$ & $\begin{array}{l}12.49 \\
12.52 \\
12.57 \\
12.61 \\
12.64\end{array}$ \\
\hline $\begin{array}{l}26 \\
27 \\
28 \\
29 \\
30 \\
31\end{array}$ & $\begin{array}{l}14.59 \\
14.59 \\
14.60 \\
14.60 \\
14.56 \\
14.48\end{array}$ & $\begin{array}{l}14.59 \\
14.59 \\
14.57 \\
14.62 \\
14.61\end{array}$ & $\begin{array}{l}14.74 \\
14.74 \\
14.74 \\
14.72 \\
14.70 \\
14.70\end{array}$ & $\begin{array}{l}14.54 \\
14.52 \\
14.58 \\
14.55 \\
14.52 \\
14.62\end{array}$ & $\begin{array}{l}14.41 \\
14.43 \\
14.47\end{array}$ & $\begin{array}{l}13.41 \\
13.49 \\
13.57 \\
13.63 \\
13.69 \\
13.74\end{array}$ & $\begin{array}{l}14.03 \\
14.05 \\
14.05 \\
14.00 \\
13.90\end{array}$ & $\begin{array}{l}12.72 \\
12.84 \\
12.95 \\
13.05 \\
13.15 \\
13.24\end{array}$ & $\begin{array}{l}12.32 \\
12.49 \\
12.65 \\
12.80 \\
12.92\end{array}$ & $\begin{array}{l}13.81 \\
13.73 \\
12.36 \\
11.72 \\
11.82 \\
12.11\end{array}$ & \begin{tabular}{c}
2.45 \\
\hdashline-- \\
--- \\
--- \\
--
\end{tabular} & $\begin{array}{l}12.70 \\
12.72 \\
12.69 \\
12.67 \\
12.68\end{array}$ \\
\hline
\end{tabular}

Well BS3-C (fig. 2)

\begin{tabular}{|c|c|c|c|c|c|c|c|c|c|c|c|}
\hline $\begin{array}{l}--- \\
--- \\
--- \\
---\end{array}$ & $\begin{array}{l}14.12 \\
14.20 \\
14.14 \\
13.84 \\
13.48\end{array}$ & $\begin{array}{r}13.57 \\
13.54 \\
- \\
- \\
--\end{array}$ & 14.62 & $\overline{--}$ & $\begin{array}{l}--- \\
--- \\
--- \\
--\end{array}$ & $\begin{array}{l}--- \\
--- \\
--- \\
---\end{array}$ & $\begin{array}{l}--- \\
--- \\
--- \\
---\end{array}$ & $\begin{array}{r}11.64 \\
11.51 \\
11.57 \\
11.76 \\
-\end{array}$ & $\begin{array}{l}10.68 \\
10.81 \\
10.86 \\
11.09 \\
11.52\end{array}$ & $\begin{array}{l}12.25 \\
12.66 \\
12.71 \\
12.84 \\
13.09\end{array}$ & $\begin{array}{l}10.54 \\
10.88 \\
11.03 \\
11.17 \\
11.58\end{array}$ \\
\hline $\begin{array}{l}14 \cdot 20 \\
14 \cdot 32 \\
14 \cdot 36 \\
14 \cdot 38 \\
14.31\end{array}$ & $\begin{array}{l}13.48 \\
13.40 \\
13.13 \\
12.91 \\
12.94\end{array}$ & $\begin{array}{l}--- \\
--- \\
--- \\
---\end{array}$ & $\begin{array}{l}--- \\
--- \\
--- \\
---\end{array}$ & $\begin{array}{l}--- \\
\overline{--} \\
\overline{--} \\
\overline{--}\end{array}$ & $\begin{array}{l}--- \\
--- \\
--- \\
---\end{array}$ & $\begin{array}{c}--- \\
--- \\
--- \\
--- \\
\end{array}$ & 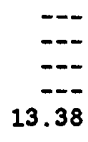 & $\begin{array}{l}m- \\
-\cdots \\
--\end{array}$ & $\begin{array}{l}11.80 \\
11.87 \\
11.90 \\
12.15 \\
12.37\end{array}$ & $\begin{array}{l}13.30 \\
13.37 \\
13.42 \\
13.52 \\
13.58\end{array}$ & $\begin{array}{l}11.62 \\
11.88 \\
12.00 \\
12.08 \\
12.28\end{array}$ \\
\hline $\begin{array}{l}14.28 \\
14.29 \\
14.39 \\
14.39 \\
14.33\end{array}$ & $\begin{array}{l}12.93 \\
13.12 \\
12.90 \\
12.86 \\
12.84\end{array}$ & $\begin{array}{l}-- \\
--- \\
---\end{array}$ & $=-$ & $\overline{-\square}$ & $\begin{array}{l}--- \\
--- \\
--- \\
--\end{array}$ & $\begin{array}{l}--- \\
--- \\
--- \\
---\end{array}$ & $\begin{array}{l}13.64 \\
13.71 \\
13.68 \\
13.61 \\
13.49\end{array}$ & $\begin{array}{l}--- \\
-- \\
--- \\
--\end{array}$ & $\begin{array}{l}12.47 \\
12.61 \\
12.78 \\
12.73 \\
12.76\end{array}$ & $\begin{array}{l}13.65 \\
13.79 \\
13.61 \\
12.90 \\
12.99\end{array}$ & $\begin{array}{l}12.38 \\
12.44 \\
12.44 \\
12.55 \\
12.64\end{array}$ \\
\hline $\begin{array}{l}14.34 \\
14.54 \\
14.76 \\
14.84 \\
14.64\end{array}$ & $\begin{array}{l}12.91 \\
12.84 \\
13.03 \\
13.03 \\
12.89\end{array}$ & $\begin{array}{l}--- \\
--- \\
--- \\
--\end{array}$ & $\begin{array}{l}--- \\
--- \\
--- \\
--\end{array}$ & $\begin{array}{l}\overline{-} \\
\overline{-} \\
\overline{-} \\
\overline{-}\end{array}$ & $\begin{array}{l}--- \\
--- \\
--- \\
--\end{array}$ & $\begin{array}{c}--- \\
-\cdots \\
-\cdots \\
--- \\
--\end{array}$ & $\begin{array}{l}13.28 \\
13.27 \\
13.39 \\
13.29 \\
12.90\end{array}$ & $\begin{array}{l}--- \\
--- \\
---\end{array}$ & $\begin{array}{l}12.90 \\
13.03 \\
13.16 \\
13.30 \\
13.24\end{array}$ & $\begin{array}{l}13.06 \\
13.11 \\
13.23 \\
13.37 \\
13.49\end{array}$ & $\begin{array}{l}12.84 \\
13.08 \\
13.09 \\
13.13 \\
13.23\end{array}$ \\
\hline $\begin{array}{l}14.46 \\
14.47 \\
14.48 \\
14.50 \\
14.49\end{array}$ & $\begin{array}{l}13.07 \\
13.14 \\
13.22 \\
13.13 \\
12.95\end{array}$ & $\begin{array}{l}--. \\
--- \\
--- \\
--\end{array}$ & $\cdots$ & $\begin{array}{l}-- \\
\overline{-} \\
\overline{-} \\
\overline{-}\end{array}$ & $\begin{array}{l}--- \\
--- \\
--- \\
---\end{array}$ & $\begin{array}{l}--- \\
--- \\
-\cdots \\
--- \\
--\end{array}$ & $\begin{array}{l}12.07 \\
11.39 \\
10.90 \\
10.73 \\
10.67\end{array}$ & $\begin{array}{l}--- \\
--- \\
--- \\
--\end{array}$ & $\begin{array}{l}12.56 \\
12.07 \\
11.95 \\
11.95 \\
12.07\end{array}$ & $\begin{array}{l}13.50 \\
13.35 \\
13.17 \\
12.85 \\
11.58\end{array}$ & $\begin{array}{l}13.25 \\
13.39 \\
13.60 \\
13.62 \\
13.55\end{array}$ \\
\hline $\begin{array}{l}14.46 \\
14.42 \\
14.36 \\
14.27 \\
14.16 \\
14.07\end{array}$ & $\begin{array}{l}13.08 \\
12.96 \\
13.23 \\
13.54 \\
13.56\end{array}$ & $\begin{array}{l}--- \\
=-- \\
=-- \\
--- \\
---\end{array}$ & $\begin{array}{l}--- \\
=- \\
=- \\
--- \\
---\end{array}$ & $\overline{--}$ & $\begin{array}{l}--- \\
=- \\
--- \\
---\end{array}$ & $\begin{array}{l}--- \\
\overline{--} \\
\overline{-} \\
-\end{array}$ & $\begin{array}{l}10.81 \\
10.99 \\
11.18 \\
11.39 \\
11.60 \\
11.67\end{array}$ & 10.44 & $\begin{array}{l}12.14 \\
12.19 \\
12.09 \\
11.78 \\
11.68 \\
11.76\end{array}$ & $\begin{array}{r}10.47 \\
10.00 \\
9.88 \\
9.96 \\
10.10 \\
10.25\end{array}$ & $\begin{array}{l}13.69 \\
13.82 \\
13.94 \\
14.05 \\
14.13\end{array}$ \\
\hline
\end{tabular}


Table 20. Daily mean water levels in unconsolidated aquifers, Clayton County, Iowa, water year 1990--Continued

\begin{tabular}{|c|c|c|c|c|c|c|c|c|c|c|c|c|}
\hline Day & Oct. & Nov. & Dec. & Jan. & Fab. & Mar. & Apr. & May & June & July & Aug. & Sept \\
\hline \multicolumn{13}{|c|}{ Well BS4-B (fig. 2) } \\
\hline $\begin{array}{l}1 \\
2 \\
3 \\
4 \\
5\end{array}$ & $\begin{array}{l}73.08 \\
73.12 \\
73.19 \\
73.17 \\
73.03\end{array}$ & $\begin{array}{l}73.09 \\
73.04 \\
73.04 \\
73.12 \\
73.07\end{array}$ & $\begin{array}{l}73.27 \\
73.27 \\
73.32 \\
73.21 \\
73.24\end{array}$ & $\begin{array}{l}73.49 \\
73.47 \\
73.46 \\
73.48 \\
73.50\end{array}$ & $\begin{array}{r}73.61 \\
73.62 \\
73.62 \\
73.61 \\
-.-\end{array}$ & $\begin{array}{l}73.72 \\
73.68 \\
73.71 \\
73.71 \\
73.74\end{array}$ & $\begin{array}{l}73.46 \\
73.47 \\
73.50 \\
73.45 \\
73.47\end{array}$ & $\begin{array}{l}--- \\
-- \\
--- \\
--\end{array}$ & $\overline{--}$ & $\begin{array}{l}73.05 \\
73.07 \\
73.06 \\
73.12 \\
73.21\end{array}$ & $\begin{array}{l}-- \\
-- \\
-- \\
--\end{array}$ & $\begin{array}{r}-- \\
\overline{--} \\
\overline{--} \\
72.64\end{array}$ \\
\hline $\begin{array}{r}6 \\
7 \\
8 \\
9 \\
10\end{array}$ & $\begin{array}{l}73.04 \\
73.09 \\
73.09 \\
73.07 \\
73.07\end{array}$ & $\begin{array}{l}73.12 \\
73.09 \\
73.06 \\
73.05 \\
73.12\end{array}$ & $\begin{array}{l}73.30 \\
73.38 \\
73.38 \\
73.29 \\
73.33\end{array}$ & $\begin{array}{l}73.50 \\
73.47 \\
73.44 \\
73.45 \\
73.49\end{array}$ & $\begin{array}{r}--- \\
73.62 \\
73.59 \\
73.60 \\
73.61\end{array}$ & $\begin{array}{l}73.78 \\
73.78 \\
73.69 \\
73.65 \\
73.67\end{array}$ & $\begin{array}{l}73.50 \\
73.54 \\
73.53 \\
73.52 \\
73.49\end{array}$ & $\begin{array}{l}--- \\
--- \\
---\end{array}$ & $\begin{array}{l}73.36 \\
73.40 \\
73.39 \\
73.42 \\
73.47\end{array}$ & $\begin{array}{l}73.26 \\
73.23 \\
73.24 \\
73.29 \\
73.31\end{array}$ & $\overline{--}$ & $\begin{array}{l}72.68 \\
72.73 \\
72.75 \\
72.77 \\
72.83\end{array}$ \\
\hline $\begin{array}{l}11 \\
12 \\
13 \\
14 \\
15\end{array}$ & $\begin{array}{l}73.06 \\
73.12 \\
73.14 \\
73.12 \\
73.11\end{array}$ & $\begin{array}{l}73.12 \\
73.19 \\
73.11 \\
73.15 \\
73.15\end{array}$ & $\begin{array}{l}73.38 \\
73.37 \\
73.34 \\
73.36 \\
73.39\end{array}$ & $\begin{array}{l}73.46 \\
73.53 \\
73.51 \\
73.49 \\
73.54\end{array}$ & $\begin{array}{l}73.61 \\
73.59 \\
73.61 \\
73.67 \\
73.60\end{array}$ & $\begin{array}{l}73.64 \\
73.56 \\
73.53 \\
73.38 \\
73.23\end{array}$ & $\begin{array}{l}73.50 \\
73.51 \\
73.46 \\
73.45 \\
73.47\end{array}$ & 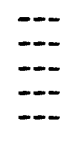 & $\begin{array}{l}73.50 \\
73.44 \\
73.40 \\
73.40 \\
73.35\end{array}$ & $\begin{array}{l}73.32 \\
73.33 \\
73.36 \\
73.32 \\
73.34\end{array}$ & \begin{tabular}{r}
73.34 \\
-0. \\
\hdashline- \\
--
\end{tabular} & $\begin{array}{l}72.84 \\
72.85 \\
72.84 \\
72.88 \\
72.89\end{array}$ \\
\hline $\begin{array}{l}16 \\
17 \\
18 \\
19 \\
20\end{array}$ & $\begin{array}{r}73.14 \\
73.20 \\
73.17 \\
73.09 \\
73.08\end{array}$ & $\begin{array}{l}73.18 \\
73.16 \\
73.23 \\
73.18 \\
73.21\end{array}$ & $\begin{array}{l}73.42 \\
73.41 \\
73.42 \\
73.42 \\
73.44\end{array}$ & $\begin{array}{l}73.52 \\
73.55 \\
73.59 \\
73.54 \\
73.47\end{array}$ & $\begin{array}{l}73.58 \\
73.68 \\
73.63 \\
73.68 \\
73.72\end{array}$ & $\begin{array}{l}73.25 \\
73.25 \\
73.29 \\
73.37 \\
73.40\end{array}$ & $\begin{array}{l}73.46 \\
73.52 \\
73.53 \\
73.47 \\
73.48\end{array}$ & $\begin{array}{l}--- \\
-- \\
--\end{array}$ & $\begin{array}{l}73.25 \\
73.16 \\
73.14 \\
73.09 \\
73.07\end{array}$ & $\begin{array}{l}73.38 \\
73.41 \\
73.40 \\
73.31 \\
73.31\end{array}$ & $\bar{m}=-$ & $\begin{array}{l}72.96 \\
72.97 \\
72.92 \\
72.95 \\
72.95\end{array}$ \\
\hline $\begin{array}{l}21 \\
22 \\
23 \\
24 \\
25\end{array}$ & $\begin{array}{l}73.15 \\
73.17 \\
73.20 \\
73.20 \\
73.20\end{array}$ & $\begin{array}{l}73.24 \\
73.24 \\
73.24 \\
73.19 \\
73.20\end{array}$ & $\begin{array}{l}73.49 \\
73.50 \\
73.45 \\
73.40 \\
73.41\end{array}$ & $\begin{array}{l}73.48 \\
73.50 \\
73.47 \\
73.53 \\
73.54\end{array}$ & $\begin{array}{l}73.66 \\
73.62 \\
73.63 \\
73.69 \\
73.77\end{array}$ & $\begin{array}{l}73.36 \\
73.34 \\
73.42 \\
73.45 \\
73.43\end{array}$ & $\begin{array}{l}73.49 \\
73.47 \\
73.50 \\
73.52 \\
73.51\end{array}$ & $\begin{array}{l}--- \\
--- \\
---\end{array}$ & $\begin{array}{l}73.11 \\
73.03 \\
72.97 \\
72.96 \\
72.94\end{array}$ & $\begin{array}{l}73.33 \\
73.34 \\
73.36 \\
73.38 \\
73.40\end{array}$ & $\begin{array}{l}--- \\
-- \\
-- \\
--\end{array}$ & $\begin{array}{l}72.96 \\
72.99 \\
73.02 \\
72.98 \\
72.99\end{array}$ \\
\hline $\begin{array}{l}26 \\
27 \\
28 \\
29 \\
30 \\
31\end{array}$ & $\begin{array}{l}73.20 \\
73.17 \\
73.12 \\
73.09 \\
73.11 \\
73.12\end{array}$ & $\begin{array}{l}73.25 \\
73.18 \\
73.29 \\
73.28 \\
73.27\end{array}$ & $\begin{array}{l}73.43 \\
73.44 \\
73.45 \\
73.47 \\
73.46 \\
73.45\end{array}$ & $\begin{array}{l}73.53 \\
73.60 \\
73.58 \\
73.55 \\
73.61 \\
73.58\end{array}$ & $\begin{array}{l}73.72 \\
73.72 \\
73.75\end{array}$ & $\begin{array}{l}73.44 \\
73.47 \\
73.46 \\
73.46 \\
73.46 \\
73.46\end{array}$ & $\begin{array}{l}73.48 \\
73.50 \\
73.53 \\
73.59 \\
73.59\end{array}$ & $\begin{array}{l}--- \\
-- \\
--- \\
-- \\
--\end{array}$ & $\begin{array}{l}72.95 \\
72.96 \\
72.96 \\
73.64 \\
73.01\end{array}$ & $\begin{array}{l}73.40 \\
73.34 \\
73.31 \\
73.29 \\
73.33 \\
73.37\end{array}$ & $\begin{array}{l}--- \\
-- \\
-- \\
--\end{array}$ & $\begin{array}{l}73.05 \\
73.06 \\
73.10 \\
73.12 \\
73.13\end{array}$ \\
\hline
\end{tabular}

Well BS4-C (fig. 2)

\begin{tabular}{|c|c|c|}
\hline $\begin{array}{l}56.01 \\
56.25 \\
56.28 \\
56.12 \\
56.01\end{array}$ & $\begin{array}{l}56.39 \\
56.32 \\
56.21 \\
56.18 \\
56.29\end{array}$ & $\begin{array}{r}56.39 \\
56.52 \\
56.40 \\
-\ldots\end{array}$ \\
\hline $\begin{array}{l}56.25 \\
56.22 \\
56.17 \\
56.10 \\
56.19\end{array}$ & $\begin{array}{l}56.41 \\
56.27 \\
56.25 \\
56.31 \\
56.41\end{array}$ & $\begin{array}{l}-- \\
\cdots- \\
\cdots- \\
\cdots\end{array}$ \\
\hline $\begin{array}{l}56.11 \\
56.33 \\
56.20 \\
56.17 \\
56.16\end{array}$ & $\begin{array}{l}56.39 \\
56.36 \\
56.28 \\
56.36 \\
56.39\end{array}$ & $\begin{array}{l}--- \\
\overline{--} \\
\overline{-} \\
--\end{array}$ \\
\hline $\begin{array}{l}56.35 \\
56.36 \\
56.31 \\
56.13 \\
56.09\end{array}$ & $\begin{array}{l}56.40 \\
56.35 \\
56.50 \\
56.24 \\
56.45\end{array}$ & $\begin{array}{l}\overline{-} \\
\overline{-} \\
-- \\
--\end{array}$ \\
\hline $\begin{array}{l}56.27 \\
56.29 \\
56.27 \\
56.28 \\
56.26\end{array}$ & $\begin{array}{l}56.43 \\
56.44 \\
56.37 \\
56.25 \\
56.43\end{array}$ & $\begin{array}{r}--- \\
56.43 \\
56.39 \\
56.44\end{array}$ \\
\hline $\begin{array}{l}56.27 \\
56.26 \\
56.26 \\
56.24 \\
56.25 \\
56.32\end{array}$ & $\begin{array}{l}56.45 \\
56.32 \\
56.69 \\
56.39 \\
56.42\end{array}$ & $\begin{array}{l}56.58 \\
56.56 \\
56.55 \\
56.64 \\
56.53 \\
56.54\end{array}$ \\
\hline
\end{tabular}

$56.77 \quad 56.71 \quad 56.77$

$56.78 \quad 56.74$

$\begin{array}{ll}56.73 & 56.81 \\ 56.69 & 56.94\end{array}$

$56.77 \quad 56.97$

56.74
56.63

56.80

56.79

56.82

56.64

56.90

56.63

56.81

56.94
56.73

56.92

56.85

56.69

56.69

57.06

56.96

56.72

56.87
$56.73 \quad 56.91$

$\begin{array}{ll}56.77 & 56.94 \\ 56.93 & 56.91 \\ 56.84 & 56.91 \\ 56.74 & 56.82 \\ 56.88 & 56.88 \\ 56.92 & 56.88 \\ 56.92 & 56.82 \\ 56.91 & 56.87 \\ 56.85 & 56.84 \\ 56.91 & 56.99 \\ 56.95 & 57.03 \\ 56.88 & 56.90 \\ 56.84 & 56.97 \\ 56.83 & 56.87 \\ 56.90 & 56.85 \\ 56.88 & 56.87 \\ 57.03 & 56.96 \\ 56.94 & 57.00 \\ 56.79 & 56.75 \\ 56.83 & 56.97 \\ 56.91 & 57.00 \\ 56.83 & 56.89 \\ 56.78 & 56.78 \\ 56.84 & 56.79 \\ 56.93 & 56.70 \\ 56.87 & 56.77 \\ 56.83 & 56.75 \\ 56.84 & 56.71 \\ 56.96 & 56.74 \\ 57.03 & 56.74 \\ & 56.65\end{array}$

56.55 56.47 56.76 56.79 56.50

56.69 56.66 56.66
56.62 56.62

56.73

56.58

56.52

56.67

56.64

56.53

56.50

56.62

56.43

56.48

56.34

56.41

56.40

56.33

56.25

56.21

56.14

56.20
56.18

56.08

56.00

56.14

56.23

56.15

56.01

56.01

56.17

56.12

56.20

56.15

55.99

56.05

56.17

56.20

56.17

56.17

56.21

56.20

56.24

56.25

56.25

56.24

56.22

56.24

56.34
56.34
56.30

56.26

56.26

56.32

56.46

56.42

56.32

56.29

56.31

56.33

56.36

56.39

56.42

56.41

56.39

.56 .40

56.51

56.52

56.50

56.40

56.43

56.34

56.2

56.22

56.13

56.17

56.14

56.04

56.12
56.11

55.98

55.91

55.88
55.78

55.97

55.96

55.87

55.94
55.97

55.89

55.84

55.82

55.96

56.09

55.90

55.83

55.89

55.91

56.04

55.79

55.97

56.11

56.08

56.13

56.05 
Table 20. Daily mean water levels in unconsolidated aquifers, Clayton County, Iowa, water year 1990--Continued

\begin{tabular}{|c|c|c|c|c|c|c|c|c|c|c|c|c|}
\hline Day & Oct. & Nov. & Dec. & Jan. & Feb. & Mar. & Apr. & May & June & July & Aug. & Sept. \\
\hline \multicolumn{13}{|c|}{ Well DCWI (fig. 2) } \\
\hline $\begin{array}{l}1 \\
2 \\
3 \\
4 \\
5\end{array}$ & $\begin{array}{l}6.13 \\
6.23 \\
6.31 \\
6.30 \\
6.10\end{array}$ & $\begin{array}{l}5.08 \\
5.12 \\
5.13 \\
5.12 \\
5.17\end{array}$ & $\begin{array}{r}5.57 \\
5.63 \\
-0 \\
5.49\end{array}$ & $\begin{array}{l}5.79 \\
5.79 \\
5.76 \\
5.82 \\
5.84\end{array}$ & $\begin{array}{l}5.83 \\
5.87 \\
5.87 \\
5.87 \\
5.71\end{array}$ & $\begin{array}{l}5.61 \\
5.43 \\
5.40 \\
5.42 \\
5.51\end{array}$ & $\begin{array}{l}4.53 \\
4.52 \\
4.49 \\
4.51 \\
4.54\end{array}$ & $\begin{array}{r}--- \\
--5 \\
4.53 \\
4.46 \\
4.44\end{array}$ & $\begin{array}{l}--- \\
--- \\
--- \\
---\end{array}$ & $\begin{array}{l}4.29 \\
4.32 \\
4.33 \\
4.35 \\
4.38\end{array}$ & $\begin{array}{l}4.13 \\
4.17 \\
4.19 \\
3.78 \\
3.93\end{array}$ & $\begin{array}{l}4.12 \\
4.14 \\
4.15 \\
4.15 \\
4.17\end{array}$ \\
\hline $\begin{array}{r}6 \\
7 \\
8 \\
9 \\
10\end{array}$ & $\begin{array}{l}6.03 \\
6.11 \\
6.12 \\
6.10 \\
6.12\end{array}$ & $\begin{array}{l}5.29 \\
5.26 \\
5.19 \\
5.28 \\
5.29\end{array}$ & $\begin{array}{l}5.60 \\
5.72 \\
5.70 \\
5.56 \\
5.61\end{array}$ & $\begin{array}{l}5.84 \\
5.80 \\
5.76 \\
5.43 \\
5.42\end{array}$ & $\begin{array}{l}5.41 \\
5.41 \\
5.17 \\
5.09 \\
5.24\end{array}$ & $\begin{array}{l}5.64 \\
5.66 \\
5.02 \\
4.84 \\
4.73\end{array}$ & $\begin{array}{l}4.55 \\
4.54 \\
4.53 \\
4.34 \\
4.36\end{array}$ & $\begin{array}{l}4.46 \\
4.46 \\
4.49 \\
4.37 \\
4.28\end{array}$ & $\begin{array}{l}4.23 \\
4.21 \\
4.21 \\
4.23 \\
4.24\end{array}$ & $\begin{array}{l}4.39 \\
4.39 \\
4.40 \\
4.43 \\
4.44\end{array}$ & $\begin{array}{l}4.05 \\
4.10 \\
4.14 \\
4.17 \\
4.21\end{array}$ & $\begin{array}{l}4.22 \\
4.25 \\
4.25 \\
4.25 \\
4.30\end{array}$ \\
\hline $\begin{array}{l}11 \\
12 \\
13 \\
14 \\
15\end{array}$ & $\begin{array}{l}6.11 \\
6.21 \\
6.22 \\
6.21 \\
6.20\end{array}$ & $\begin{array}{l}5.38 \\
5.27 \\
5.35 \\
5.37 \\
5.41\end{array}$ & $\begin{array}{c}5.71 \\
--- \\
--- \\
---\end{array}$ & $\begin{array}{l}5.51 \\
5.70 \\
5.73 \\
5.69 \\
5.76\end{array}$ & $\begin{array}{l}5.40 \\
5.39 \\
5.35 \\
5.48 \\
5.45\end{array}$ & $\begin{array}{l}4.16 \\
3.88 \\
3.99 \\
3.82 \\
3.96\end{array}$ & $\begin{array}{l}4.38 \\
4.38 \\
4.37 \\
4.36 \\
4.36\end{array}$ & $\begin{array}{l}4.31 \\
4.32 \\
4.34 \\
4.34 \\
4.36\end{array}$ & $\begin{array}{l}4.24 \\
4.20 \\
3.89 \\
3.97 \\
3.89\end{array}$ & $\begin{array}{l}4.44 \\
4.44 \\
4.44 \\
4.42 \\
4.43\end{array}$ & $\begin{array}{l}4.25 \\
4.30 \\
4.33 \\
4.33 \\
4.35\end{array}$ & $\begin{array}{l}4.32 \\
4.33 \\
4.34 \\
4.35 \\
4.36\end{array}$ \\
\hline $\begin{array}{l}16 \\
17 \\
18 \\
19 \\
20\end{array}$ & $\begin{array}{l}5.72 \\
5.58 \\
5.71 \\
5.69 \\
5.60\end{array}$ & $\begin{array}{l}5.40 \\
5.48 \\
5.43 \\
5.45 \\
5.49\end{array}$ & $\begin{array}{c}--- \\
-\cdots \\
0.05\end{array}$ & $\begin{array}{l}5.78 \\
5.41 \\
5.53 \\
5.62 \\
5.59\end{array}$ & $\begin{array}{l}5.49 \\
5.68 \\
5.62 \\
5.72 \\
5.74\end{array}$ & $\begin{array}{l}4.20 \\
4.31 \\
4.42 \\
4.49 \\
4.50\end{array}$ & $\begin{array}{l}4.38 \\
4.39 \\
4.37 \\
4.35 \\
4.34\end{array}$ & $\begin{array}{l}4.35 \\
4.36 \\
4.38 \\
3.90 \\
3.78\end{array}$ & $\begin{array}{l}3.76 \\
3.61 \\
3.76 \\
4.04 \\
4.10\end{array}$ & $\begin{array}{l}4.46 \\
4.50 \\
4.46 \\
3.99 \\
3.86\end{array}$ & $\begin{array}{l}4.37 \\
4.36 \\
4.35 \\
3.84 \\
3.78\end{array}$ & $\begin{array}{l}4.40 \\
4.43 \\
4.40 \\
4.39 \\
4.39\end{array}$ \\
\hline $\begin{array}{l}21 \\
22 \\
23 \\
24 \\
25\end{array}$ & $\begin{array}{l}5.68 \\
5.79 \\
5.81 \\
5.84 \\
5.85\end{array}$ & $\begin{array}{l}5.50 \\
5.51 \\
5.46 \\
5.46 \\
5.52\end{array}$ & $\begin{array}{l}5.64 \\
5.47 \\
5.34 \\
5.16 \\
5.06\end{array}$ & $\begin{array}{l}5.63 \\
5.70 \\
5.65 \\
5.72 \\
5.74\end{array}$ & $\begin{array}{l}5.68 \\
5.54 \\
5.49 \\
5.62 \\
5.71\end{array}$ & $\begin{array}{l}4.50 \\
4.50 \\
4.48 \\
4.49 \\
4.50\end{array}$ & $\begin{array}{l}4.34 \\
4.31 \\
4.32 \\
4.36 \\
4.25\end{array}$ & $\begin{array}{l}4.02 \\
4.13 \\
4.16 \\
4.23 \\
4.25\end{array}$ & $\begin{array}{l}4.14 \\
3.76 \\
3.80 \\
3.98 \\
4.09\end{array}$ & $\begin{array}{l}4.07 \\
4.16 \\
4.23 \\
4.30 \\
4.33\end{array}$ & $\begin{array}{l}3.83 \\
3.91 \\
3.97 \\
3.63 \\
3.09\end{array}$ & $\begin{array}{l}4.39 \\
4.42 \\
4.45 \\
4.44 \\
4.45\end{array}$ \\
\hline $\begin{array}{l}26 \\
27 \\
28 \\
29 \\
30 \\
31\end{array}$ & $\begin{array}{l}5.86 \\
5.86 \\
5.85 \\
5.81 \\
5.48 \\
5.04\end{array}$ & $\begin{array}{l}5.44 \\
5.57 \\
5.57 \\
5.55 \\
5.54\end{array}$ & $\begin{array}{l}5.21 \\
5.39 \\
5.51 \\
5.65 \\
5.71 \\
5.71\end{array}$ & $\begin{array}{l}5.76 \\
5.77 \\
5.86 \\
5.80 \\
5.81 \\
5.85\end{array}$ & $\begin{array}{l}5.65 \\
5.70 \\
5.74\end{array}$ & $\begin{array}{l}4.54 \\
4.53 \\
4.53 \\
4.53 \\
4.53 \\
4.53\end{array}$ & $\begin{array}{l}4.29 \\
4.36 \\
4.37 \\
4.42 \\
4.44\end{array}$ & $\begin{array}{r}4.25 \\
- \\
- \\
- \\
-\end{array}$ & $\begin{array}{l}4.14 \\
4.18 \\
4.22 \\
4.25 \\
4.27\end{array}$ & $\begin{array}{l}4.35 \\
3.82 \\
3.82 \\
3.90 \\
3.98 \\
4.09\end{array}$ & $\begin{array}{l}3.61 \\
3.79 \\
3.90 \\
3.97 \\
4.04 \\
4.08\end{array}$ & $\begin{array}{l}4.49 \\
4.50 \\
4.50 \\
4.51 \\
4.50\end{array}$ \\
\hline
\end{tabular}


Table 21. Onsite measurements of selected water-quality constituents at selected ground-water monitoring sites in the Big Spring basin, Clayton County, Iowa, water year 1990

$\left[\mu \mathrm{S} / \mathrm{cm}\right.$, microsiemens per centimeter at 25 degrees Celsius; ${ }^{\circ} \mathrm{C}$, degrees Celsius; mg/L, milligrams per liter; $\mathrm{ft}^{3} / \mathrm{s}$, cubic feet per second; --, missing data]

\begin{tabular}{|c|c|c|c|c|c|c|}
\hline Date & $\begin{array}{c}\text { Time } \\
(24-\text { hour })\end{array}$ & $\begin{array}{l}\text { Water } \\
\text { level, - } \\
\text { (feet } \\
\text { below } \\
\text { land } \\
\text { surface) }\end{array}$ & $\begin{array}{l}\text { Spe- } \\
\text { cific } \\
\text { con- } \\
\text { duct- } \\
\text { ance } \\
(\mu \mathrm{S} / \mathrm{cm})\end{array}$ & $\begin{array}{c}\mathrm{pH} \\
\text { (standard } \\
\text { units) }\end{array}$ & $\begin{array}{l}\text { Water } \\
\text { temper- } \\
\text { ature } \\
\left({ }^{\circ} \mathrm{C}\right)\end{array}$ & $\begin{array}{l}\text { Dissolved } \\
\text { oxygen } \\
(\mathrm{mg} / \mathrm{L})\end{array}$ \\
\hline
\end{tabular}

Deer Creek well (DCW2, fig. 2)

$\begin{array}{lllllll}10-04-89 & 1300 & 7.27 & 811 & 6.9 & 13.5 & -. \\ 11-08-89 & 1000 & 6.30 & 763 & 6.9 & 13.0 & \ldots \\ 12-05-89 & 1230 & 6.53 & 780 & 7.0 & 10.0 & -. \\ 01-04-90 & 1100 & 6.90 & 720 & 7.0 & 9.0 & -- \\ 02-07-90 & 0940 & 6.53 & 730 & 7.0 & 7.0 & 1.8 \\ 03-06-90 & 1000 & 6.69 & 730 & 7.1 & 6.0 & 13.0 \\ 03-21-90 & 1500 & 5.60 & 784 & 7.0 & 7.0 & -- \\ 04-03-90 & 1000 & 5.61 & 725 & 7.0 & 5.0 & -- \\ 05-02-90 & 1100 & 5.52 & 750 & 7.0 & 7.0 & 1.2 \\ 06-05-90 & 1300 & 5.19 & 742 & 6.8 & 8.0 & 0.8 \\ 07-02-90 & 1115 & 5.93 & 600 & 7.4 & 17.0 & 9.5 \\ 08-02-90 & 0850 & 5.74 & 592 & -. & 13.5 & 10.1 \\ 08-20-90 & 1410 & 5.40 & 606 & -. & 18.0 & -. \\ 08-26-90 & 1340 & -. & 680 & 7.5 & 22.0 & -. \\ 09-06-90 & 0750 & 5.76 & 615 & 7.8 & 17.0 & 6.7\end{array}$

Deer Creek well (DCW3, fig. 2))

\begin{tabular}{|c|c|c|c|c|c|c|}
\hline $\begin{array}{l}10-05-89 \\
11-08-89 \\
12-05-89 \\
01-04=90 \\
02-07-90\end{array}$ & $\begin{array}{l}0900 \\
1130 \\
1330 \\
1220 \\
0830\end{array}$ & $\begin{array}{l}9.51 \\
9.60 \\
8.96 \\
9.19 \\
9.29\end{array}$ & $\begin{array}{l}647 \\
635 \\
590 \\
635 \\
590\end{array}$ & $\begin{array}{l}7.2 \\
7.2 \\
7.2 \\
7.2 \\
7.1\end{array}$ & $\begin{array}{r}13.5 \\
12.0 \\
11.0 \\
8.0 \\
7.0\end{array}$ & $\begin{array}{l}-- \\
-- \\
-- \\
-- \\
2.7\end{array}$ \\
\hline $\begin{array}{l}03-06-90 \\
03-21-90 \\
04-03-90 \\
05-02-90 \\
06-05-90\end{array}$ & $\begin{array}{l}1110 \\
1540 \\
1130 \\
1200 \\
1150\end{array}$ & $\begin{array}{l}9.34 \\
7.60 \\
8.04 \\
8.28 \\
6.94\end{array}$ & $\begin{array}{l}645 \\
658 \\
620 \\
672 \\
653\end{array}$ & $\begin{array}{l:l}7 & .2 \\
7 & .0 \\
7 & .2 \\
7 & .2 \\
7 & .0\end{array}$ & $\begin{array}{l}7.0 \\
7.0 \\
7.0 \\
8.0 \\
7.0\end{array}$ & $\begin{array}{l}2.3 \\
-- \\
-- \\
1.7 \\
1.4\end{array}$ \\
\hline $\begin{array}{l}07-02-90 \\
08-02-90 \\
09-06-90\end{array}$ & $\begin{array}{l}1215 \\
1130 \\
1030\end{array}$ & $\begin{array}{l}5.59 \\
5.48 \\
5.07\end{array}$ & $\begin{array}{l}515 \\
605 \\
600\end{array}$ & $\begin{array}{l}6.5 \\
--1 \\
7.1\end{array}$ & $\begin{array}{l}11.0 \\
13.0 \\
14.0\end{array}$ & $\begin{array}{l}1.2 \\
1.6 \\
1.2\end{array}$ \\
\hline
\end{tabular}


Table 21. Onsite measurements of selected water-quality constituents at selected ground-water monitoring sites in the Big Spring basin, Clayton County, Iowa, water year 1990--Continued

\begin{tabular}{|c|c|c|c|c|c|c|}
\hline Date & $\begin{array}{c}\text { Time } \\
\text { (24-hour) }\end{array}$ & $\begin{array}{l}\text { Instan- } \\
\text { taneous } \\
\text { dis- } \\
\text { charge } \\
\left(\mathrm{ft} \mathrm{t}^{3} / \mathrm{s}\right)\end{array}$ & $\begin{array}{l}\text { Spe- } \\
\text { cific } \\
\text { con- } \\
\text { duct- } \\
\text { ance } \\
(\mu \mathrm{S} / \mathrm{cm})\end{array}$ & $\begin{array}{c}\mathrm{pH} \\
\text { (standard } \\
\text { units) }\end{array}$ & $\begin{array}{l}\text { Water } \\
\text { temper- } \\
\text { ature } \\
\left({ }^{\circ} \mathrm{C}\right)\end{array}$ & $\begin{array}{l}\text { Dissolv } \\
\text { oxygen } \\
\text { (mg/L) }\end{array}$ \\
\hline & Dee & \multicolumn{2}{|c|}{ Creek tile line } & (DCT2, $\mathrm{fi}$ & ig. 2) & \\
\hline $\begin{array}{l}11-08-89 \\
12-05-89 \\
01-04-90 \\
02-07-90 \\
03-06-90\end{array}$ & $\begin{array}{l}0915 \\
0900 \\
0820 \\
1030 \\
0800\end{array}$ & $\begin{array}{l}0.003 \\
.0006 \\
.0005 \\
.002 \\
.0008\end{array}$ & $\begin{array}{l}475 \\
510 \\
567 \\
518 \\
590\end{array}$ & $\begin{array}{l}6.3 \\
7.0 \\
6.9 \\
6.7 \\
7.0\end{array}$ & $\begin{array}{r}10.5 \\
7.0 \\
6.0 \\
4.0 \\
3.0\end{array}$ & $\begin{array}{l}-- \\
-- \\
-- \\
-- \\
--\end{array}$ \\
\hline $\begin{array}{l}04-03-90 \\
05-02-90 \\
06-05-90 \\
07-02-90 \\
08-02-90\end{array}$ & $\begin{array}{l}0815 \\
0815 \\
1100 \\
0940 \\
0815\end{array}$ & $\begin{array}{l}.002 \\
.0006 \\
.003 \\
.001 \\
.004\end{array}$ & $\begin{array}{l}700 \\
621 \\
900 \\
770 \\
740\end{array}$ & $\begin{array}{l}6.7 \\
6.8 \\
-- \\
5.9 \\
-.\end{array}$ & $\begin{array}{c}4.0 \\
7.0 \\
-- \\
17.0 \\
14.0\end{array}$ & $\begin{array}{l}-- \\
-- \\
-- \\
--\end{array}$ \\
\hline $09-06-90$ & 0730 & $\begin{array}{l}.004 \\
\text { Big }\end{array}$ & $\begin{array}{r}705 \\
\text { ring }\end{array}$ & $\begin{array}{r}6.6 \\
i g .2)\end{array}$ & 17.0 & -- \\
\hline $\begin{array}{l}10-05-89 \\
11-08-89 \\
12-05-89 \\
01-04-90 \\
01-22-90\end{array}$ & $\begin{array}{l}1145 \\
1500 \\
1400 \\
1300 \\
1125\end{array}$ & $\begin{array}{l}-- \\
-- \\
-- \\
--\end{array}$ & $\begin{array}{l}735 \\
680 \\
700 \\
715 \\
635\end{array}$ & $\begin{array}{l}7.1 \\
7.1 \\
7.3 \\
7.3 \\
5.0\end{array}$ & $\begin{array}{r}11.0 \\
10.0 \\
10.0 \\
10.0 \\
9.5\end{array}$ & $\begin{array}{c}-. \\
\cdots \\
10.6 \\
9.6 \\
--\end{array}$ \\
\hline $\begin{array}{l}02-06-90 \\
02-08-90 \\
02-12-90 \\
03-05-90 \\
03-08-90\end{array}$ & $\begin{array}{l}1445 \\
1545 \\
1105 \\
1245 \\
1730\end{array}$ & $\begin{array}{l}23 \\
38 \\
-- \\
14 \\
--\end{array}$ & $\begin{array}{r}760 \\
\ldots- \\
-- \\
635 \\
630\end{array}$ & $\begin{array}{l}7.1 \\
7.2 \\
7.0 \\
7.1 \\
7.2\end{array}$ & $\begin{array}{l}8.5 \\
9.0 \\
8.5 \\
9.0 \\
8.5\end{array}$ & $\begin{array}{l}7.8 \\
7.2 \\
-- \\
-- \\
--\end{array}$ \\
\hline $\begin{array}{l}03-09-90 \\
03-10-90 \\
03-12-90 \\
03-16-90 \\
04-02-90\end{array}$ & $\begin{array}{l}1630 \\
0815 \\
1200 \\
1330 \\
1340\end{array}$ & $\begin{array}{l}35 \\
-- \\
-- \\
-- \\
15\end{array}$ & $\begin{array}{r}352 \\
375 \\
-- \\
568 \\
713\end{array}$ & $\begin{array}{l}7.0 \\
7.0 \\
7.1 \\
7.2 \\
7.3\end{array}$ & $\begin{array}{l}8.5 \\
6.0 \\
6.0 \\
8.0 \\
9.0\end{array}$ & $\begin{array}{l}8.2 \\
7.0 \\
7.5 \\
-. \\
--\end{array}$ \\
\hline
\end{tabular}


Table 21. Onsite measurements of selected water-quality constituents at selected ground-water monitoring sites in the Big Spring basin, Clayton County, Iowa, water year 1990--Continued

\begin{tabular}{|c|c|c|c|c|c|c|}
\hline Date & $\begin{array}{c}\text { Time } \\
\text { (24-hour) }\end{array}$ & $\begin{array}{l}\text { Instan- } \\
\text { taneous } \\
\text { dis- } \\
\text { charge } \\
\left(\mathrm{ft}^{3} / \mathrm{s}\right)\end{array}$ & $\begin{array}{l}\text { Spe- } \\
\text { cific } \\
\text { con- } \\
\text { duct- } \\
\text { ance } \\
(\mu \mathrm{S} / \mathrm{cm})\end{array}$ & $\begin{array}{c}\mathrm{pH} \\
\text { (standard } \\
\text { units) }\end{array}$ & $\begin{array}{l}\text { Water } \\
\text { temper- } \\
\text { ature } \\
\left({ }^{\circ} \mathrm{C}\right)\end{array}$ & $\begin{array}{l}\text { Dissolved } \\
\text { oxygen } \\
\text { (mg/L) }\end{array}$ \\
\hline \multicolumn{7}{|c|}{ Big Spring--Continued } \\
\hline $\begin{array}{l}05-01-90 \\
06-06-90 \\
06-22-90 \\
06-23-90 \\
07-02-90\end{array}$ & $\begin{array}{l}1410 \\
1030 \\
1700 \\
0700 \\
1600\end{array}$ & $\begin{array}{l}14 \\
20 \\
--\end{array}$ & $\begin{array}{l}710 \\
741 \\
740 \\
725 \\
748\end{array}$ & $\begin{array}{l}7.2 \\
7.0 \\
6.8 \\
6.8 \\
6.8\end{array}$ & $\begin{array}{c}9.0 \\
10.5 \\
\cdots \\
\cdots \\
10.0\end{array}$ & $\begin{array}{l}8.2 \\
8.4 \\
-. \\
-- \\
9.0\end{array}$ \\
\hline $\begin{array}{l}07-20-90 \\
08-01-90 \\
08-19-90 \\
08-19-90 \\
08-20-90\end{array}$ & $\begin{array}{l}1240 \\
1320 \\
1320 \\
1520 \\
1600\end{array}$ & $\begin{array}{l}-- \\
22 \\
-- \\
-- \\
--\end{array}$ & $\begin{array}{l}738 \\
755 \\
757 \\
756 \\
762\end{array}$ & $\begin{array}{l}6.7 \\
6.7 \\
6.7 \\
6.7 \\
6.7\end{array}$ & $\begin{array}{l}10.5 \\
10.5 \\
10.5 \\
10.5 \\
10.5\end{array}$ & $\begin{array}{l}-- \\
9.0 \\
\cdots \\
\cdots \\
\cdots\end{array}$ \\
\hline $\begin{array}{l}08-26-90 \\
08-27-90 \\
08-28-90 \\
08-30-90 \\
09-05-90\end{array}$ & $\begin{array}{l}1215 \\
1045 \\
1040 \\
1200 \\
1430\end{array}$ & $\begin{array}{l}-- \\
-- \\
-- \\
-- \\
--\end{array}$ & $\begin{array}{l}618 \\
663 \\
715 \\
756 \\
763\end{array}$ & $\begin{array}{l}6.7 \\
6.7 \\
6.7 \\
6.6 \\
6.7\end{array}$ & $\begin{array}{l}11.5 \\
11.5 \\
11.5 \\
11.0 \\
11.0\end{array}$ & $\begin{array}{l}-- \\
-- \\
-- \\
-- \\
--\end{array}$ \\
\hline
\end{tabular}


Table 22. Concentrations of major ions at selected ground-water monitoring sites in the Big Spring basin, Clayton County, Iowa, water year 1990

[Dissolved constituents are in milligrams per liter; --, data not collected]

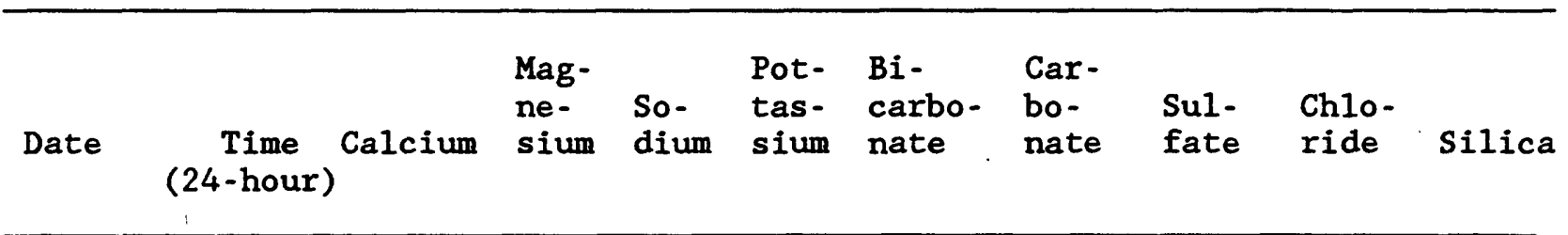

\begin{tabular}{|c|c|c|c|c|c|c|c|c|c|c|}
\hline \multirow[b]{2}{*}{$\begin{array}{l}10-04-89 \\
11-08-89 \\
12-05-89 \\
08-02-90 \\
09-06-90\end{array}$} & & & eer $C$ & reek & well & $\mathrm{W} 2$, & 2) & & & \\
\hline & $\begin{array}{l}1300 \\
1000 \\
1230 \\
1030 \\
0930\end{array}$ & $\begin{array}{l}130 \\
120 \\
120 \\
140 \\
120\end{array}$ & $\begin{array}{l}36 \\
35 \\
36 \\
37 \\
38\end{array}$ & $\begin{array}{l}7.7 \\
6.2 \\
6.8 \\
6.3 \\
7.4\end{array}$ & $\begin{array}{r}0.7 \\
1.9 \\
.6 \\
<.1 \\
.9\end{array}$ & $\begin{array}{l}-- \\
450 \\
410 \\
\cdots \\
360\end{array}$ & $\begin{array}{l}\cdots \\
0 \\
0 \\
0\end{array}$ & $\begin{array}{l}96 \\
81 \\
84 \\
81 \\
83\end{array}$ & $\begin{array}{l}27 \\
26 \\
26 \\
35 \\
38\end{array}$ & $\begin{array}{c}11 \\
11 \\
\vdots- \\
9.8 \\
11\end{array}$ \\
\hline \multicolumn{11}{|c|}{ Deer Creek well (DCW3, fig. 2) } \\
\hline $\begin{array}{l}10-05-89 \\
11-08-89 \\
12-05-89 \\
08-02-90 \\
09-06-90\end{array}$ & $\begin{array}{l}0900 \\
1130 \\
1330 \\
1215 \\
1030\end{array}$ & $\begin{array}{c}100 \\
100 \\
3.4 \\
120 \\
81\end{array}$ & $\begin{array}{l}29 \\
28 \\
1.6 \\
29 \\
28\end{array}$ & $\begin{array}{l}6.6 \\
5.7 \\
5.9 \\
5.7 \\
6.4\end{array}$ & $\begin{array}{r}2.0 \\
.6 \\
1.6 \\
1.2 \\
1.9\end{array}$ & $\begin{array}{l}-- \\
460 \\
460 \\
- \\
430\end{array}$ & $\begin{array}{l}\cdots \\
0 \\
0 \\
\cdots \\
0\end{array}$ & $\begin{array}{l}19 \\
15 \\
8.8 \\
20 \\
15\end{array}$ & $\begin{array}{l}2 \\
2.5 \\
2.5 \\
1.0 \\
1.0\end{array}$ & $\begin{array}{l}27 \\
27 \\
\therefore- \\
29 \\
28\end{array}$ \\
\hline \multicolumn{11}{|c|}{ Big Spring (fig. 2) } \\
\hline $\begin{array}{l}10-05-89 \\
12-05-89 \\
01-04-90 \\
03-12-90 \\
04-02-90\end{array}$ & $\begin{array}{l}1145 \\
1400 \\
1300 \\
1200 \\
1340\end{array}$ & $\begin{array}{l}90 \\
91 \\
84 \\
40 \\
83\end{array}$ & $\begin{array}{l}38 \\
40 \\
40 \\
16 \\
32\end{array}$ & $\begin{array}{c}21 \\
19 \\
21 \\
5.9 \\
12\end{array}$ & $\begin{array}{c}3.8 \\
3.8 \\
3.2 \\
25 \\
4.8\end{array}$ & $\begin{array}{l}390 \\
400 \\
390 \\
\ldots \\
--\end{array}$ & $\begin{array}{l}0 \\
0 \\
0 \\
\cdots \\
-.\end{array}$ & $\begin{array}{l}43 \\
36 \\
43 \\
29 \\
40\end{array}$ & $\begin{array}{l}31 \\
32 \\
15 \\
27 \\
29\end{array}$ & $\begin{array}{l}15 \\
\cdots \\
-- \\
12 \\
15\end{array}$ \\
\hline $06-06-90$ & 1030 & 85 & 36 & 14 & 3.6 & 330 & 0 & 34 & 28 & - \\
\hline
\end{tabular}


Table 23. Selected nitrogen, phosphorus, and carbon species at ground-water monitoring sites in the Big Spring basin, Clayton County, Iowa, water year 1990

[Total constituents in milligrams per liter; N, nitrogen; $P$, phosphorus; $C$, carbon; <, less than detection level indicated; --, data not collected]

\begin{tabular}{|c|c|c|c|c|c|c|}
\hline Date & $\begin{array}{c}\text { Time } \\
\text { (24-hour) }\end{array}$ & $\begin{array}{l}\text { Nitrite } \\
\text { plus } \\
\text { nitrate, } \\
\text { total } \\
\text { (as } N \text { ) }\end{array}$ & $\begin{array}{l}\text { Ammonia, } \\
\text { total } \\
(\text { as } N)\end{array}$ & $\begin{array}{l}\text { Organic } \\
\text { nitrogen, } \\
\text { total } \\
\text { (as N) }\end{array}$ & $\begin{array}{l}\text { Ortho- } \\
\text { phos - } \\
\text { phorus, } \\
\text { total } \\
\text { (as P) }\end{array}$ & $\begin{array}{l}\text { Organic } \\
\text { carbon, } \\
\text { total } \\
\text { (as C) }\end{array}$ \\
\hline
\end{tabular}

Deer Creek well (DCW2, fig. 2)

$\begin{array}{lcccccc}10-04-89 & 1300 & * 0.10 & *<0.10 & * 0.20 & *<0.10 & 2.0 \\ 11-08-89 & 1000 & *<.10 & *<.10 & *<.10 & *<.10 & 2.0 \\ 12-05-89 & 1230 & *<.10 & *<.10 & * .10 & *<.10 & 1.3 \\ 01-04-90 & 1100 & <.10 & <.10 & <.10 & <.10 & 2.3 \\ 02-07-90 & 0940 & <.10 & <.10 & .10 & <.10 & 2.6 \\ 03-06-90 & 1000 & <.10 & <.10 & .20 & <.10 & 1.7 \\ 03-21-90 & 1500 & .80 & <.10 & .20 & <.10 & 2.4 \\ 04-03-90 & 1000 & .90 & <.10 & .10 & <.10 & 1.9 \\ 05-02-90 & 1100 & .30 & <.10 & <.10 & <.10 & 1.8 \\ 06-05-90 & 1300 & 3.7 & <.10 & .20 & <.10 & 2.9 \\ 07-02-90 & 1115 & 12 & <.10 & .30 & <.10 & 3.4 \\ 08-02-90 & 1030 & 15 & .80 & .60 & .20 & 3.2 \\ 09-06-90 & 0930 & 16 & .20 & .50 & <.10 & 3.5\end{array}$

Deer Creek well (DCW3; fig. 2)

\begin{tabular}{|c|c|c|c|c|c|c|c|}
\hline $\begin{array}{l}10-05-89 \\
11-08-89 \\
12-05-89 \\
01-04-90 \\
02-07-90\end{array}$ & $\begin{array}{l}0900 \\
1130 \\
1330 \\
1220 \\
0830\end{array}$ & $\begin{array}{r}*<0.10 \\
*<.10 \\
*<.10 \\
<.10 \\
.10\end{array}$ & $\begin{array}{r}* 0.20 \\
* \quad .20 \\
*<.10 \\
<.10 \\
.20\end{array}$ & $\begin{array}{r}*<0.10 \\
*<\quad .10 \\
* \quad .10 \\
.10 \\
.50\end{array}$ & $\begin{array}{l}*<0 \\
*< \\
*< \\
<\end{array}$ & $\begin{array}{l}.10 \\
.10 \\
.10 \\
.10 \\
.30\end{array}$ & $\begin{array}{l}1.2 \\
1.8 \\
3.8 \\
2.7 \\
4.0\end{array}$ \\
\hline $\begin{array}{l}03-06-90 \\
03-21-90 \\
04-03-90 \\
05-02-90 \\
06-05-90\end{array}$ & $\begin{array}{l}1110 \\
1540 \\
1130 \\
1200 \\
1150\end{array}$ & $\begin{array}{l}<.10 \\
<.10 \\
.10 \\
<.10 \\
.10\end{array}$ & $\begin{array}{l}.10 \\
.20 \\
.20 \\
.20 \\
.20\end{array}$ & $\begin{array}{l}.20 \\
.10 \\
.30 \\
.10 \\
.30\end{array}$ & $\begin{array}{l}< \\
<\end{array}$ & $\begin{array}{l}.10 \\
.10 \\
.20 \\
.20 \\
.20\end{array}$ & $\begin{array}{l}1.4 \\
1.3 \\
1.7 \\
1.5 \\
3.4\end{array}$ \\
\hline $\begin{array}{l}07-02-90 \\
08-02-90 \\
09-06-90\end{array}$ & $\begin{array}{l}1215 \\
1130 \\
1030\end{array}$ & $\begin{array}{r}<.10 \\
<.10 \\
<.10\end{array}$ & $\begin{array}{r}.20 \\
<.10 \\
.10\end{array}$ & $\begin{array}{l}.30 \\
.10 \\
.20\end{array}$ & $\begin{array}{l}< \\
<\end{array}$ & $\begin{array}{l}.10 \\
.10 \\
.10\end{array}$ & $\begin{array}{l}4.1 \\
1.7 \\
1.5\end{array}$ \\
\hline
\end{tabular}


Table 23. Selected nitrogen, phosphorus, and carbon species at ground-water monitoring sites in the Big Spring basin, Clayton County, Iowa, water year 1990--Continued

\begin{tabular}{|c|c|c|c|c|c|c|}
\hline Date & $\begin{array}{c}\text { Time } \\
\text { (24-hour) }\end{array}$ & $\begin{array}{l}\text { Nitrite } \\
\text { plus } \\
\text { nitrate, } \\
\text { total } \\
\text { (as } N \text { ) }\end{array}$ & $\begin{array}{l}\text { Ammonia, } \\
\text { total } \\
\text { (as N) }\end{array}$ & $\begin{array}{l}\text { Organic } \\
\text { nitrogen, } \\
\text { total } \\
\text { (as } N \text { ) }\end{array}$ & $\begin{array}{l}\text { Ortho- } \\
\text { phos- } \\
\text { phorus, } \\
\text { total } \\
\text { (as P) }\end{array}$ & $\begin{array}{l}\text { Organic } \\
\text { carbon, } \\
\text { total } \\
\text { (as C) }\end{array}$ \\
\hline
\end{tabular}

Deer Creek lysimeter (DCLA, fig. 2)

$\begin{array}{llrrrrr}10-06-89 & 0950 & *<0.10 & *<0.10 & *<0.10 & *<0.10 & 1.5 \\ 03-21-90 & 1410 & <.10 & <.10 & .10 & <.10 & 1.2 \\ 04-04-90 & 0800 & <.10 & <.10 & <.10 & <.10 & 1.3 \\ 05-02-90 & 0845 & <.10 & <.10 & <.10 & <.10 & -. \\ 05-10-90 & 0810 & <.10 & <.10 & .40 & <.10 & 1.3 \\ 06-07-90 & 0810 & <.10 & <.10 & <.10 & .20 & 1.4 \\ 06-19-90 & 1201 & <.10 & <.10 & .20 & <.10 & 1.4 \\ 07-02-90 & 0910 & <.10 & <.10 & .10 & <.10 & 1.3 \\ 08-03-90 & 0830 & .10 & <.10 & .20 & <.10 & 1.5 \\ 09-07-90 & 0740 & <.10 & <.10 & <.10 & <.10 & 1.4\end{array}$

Deer Creek lysimeter (DCLB, fig. 2)

$\begin{array}{rrrrrrr}10-06-89 & 0952 & *<0.10 & * .0 .10 & * 0.10 & *<0.10 & 1.7 \\ 03-21-90 & 1411 & .10 & <.10 & .10 & <.10 & 1.8 \\ 04-04-90 & 0801 & <.10 & <.10 & <.10 & <.10 & 1.5 \\ 05-02-90 & 0846 & <.10 & <.10 & <.10 & <.10 & 1.1 \\ 05-10-90 & 0811 & <.10 & <.10 & .10 & <.10 & 1.5 \\ 06-06-90 & 0757 & <.10 & <.10 & .10 & .10 & 3.9 \\ 06-19-90 & 1202 & <.10 & <.10 & .20 & <.10 & 1.7 \\ 07-02-90 & 0911 & <.10 & <.10 & .10 & <.10 & 1.7 \\ 08-03-90 & 0831 & . .70 & <.10 & .20 & <.10 & 1.8 \\ 09-07-90 & 0742 & <.10 & <.10 & .10 & <.10 & 1.7\end{array}$


Table 23. Selected nitrogen, phosphorus, and carbon species at ground-water monitoring sites in the Big Spring basin, Clayton County, Iowa, water year 1990--Continued

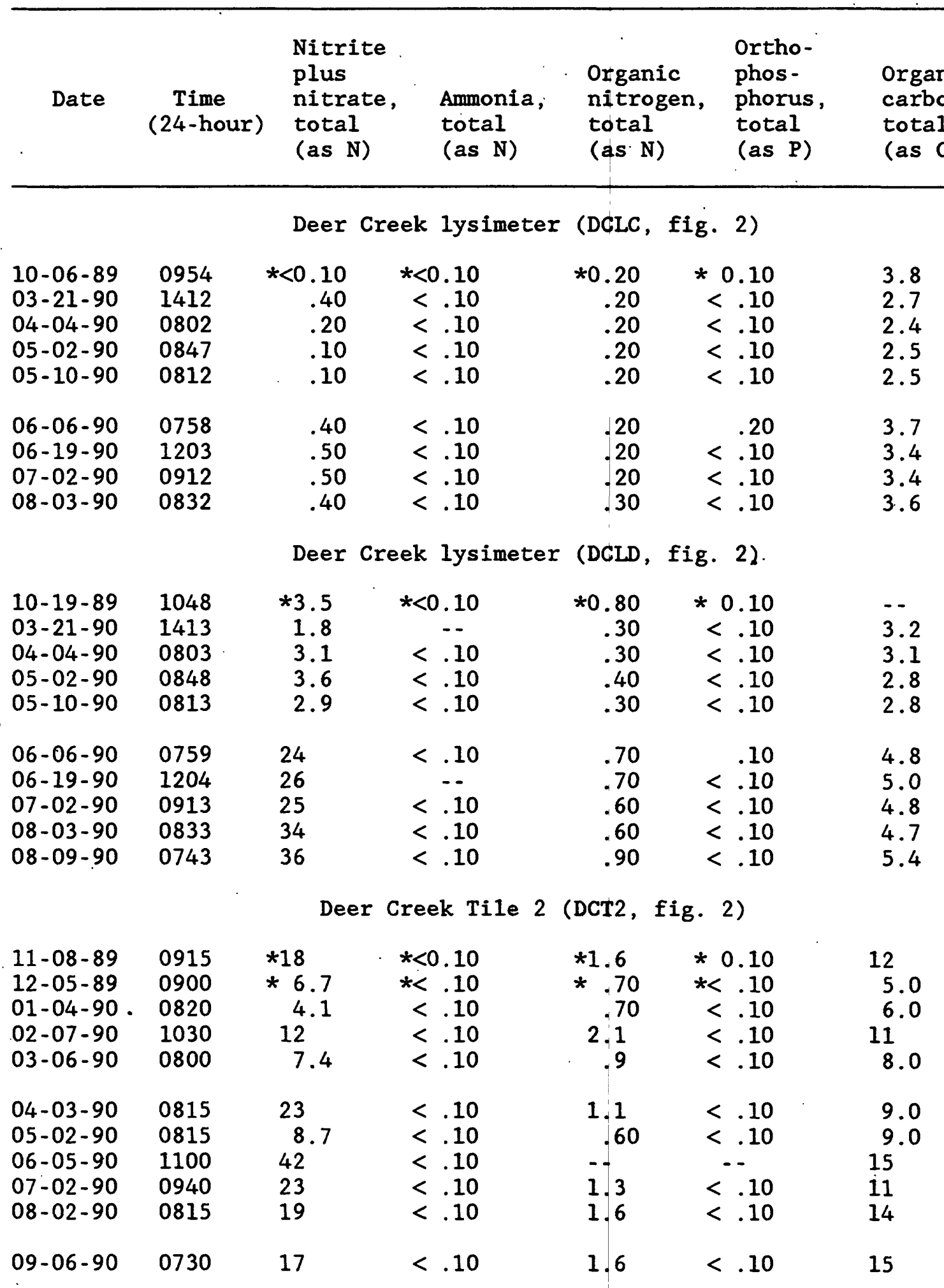


Table 23. Selected nitrogen, phosphorus, and carbon species at ground-water monitoring sites in the Big Spring basin, Clayton County, Iowa, water year 1990--Continued

\begin{tabular}{|c|c|c|c|c|c|c|}
\hline Date & $\begin{array}{c}\text { Time } \\
(24-\text { hour })\end{array}$ & $\begin{array}{l}\text { Nitrite } \\
\text { plus } \\
\text { nitrate, } \\
\text { total } \\
(\text { as } N)\end{array}$ & $\begin{array}{l}\text { Ammonia, } \\
\text { tota1 } \\
\text { (as N) }\end{array}$ & $\begin{array}{l}\text { Organic } \\
\text { nitrogen, } \\
\text { total } \\
\text { (as } N \text { ) }\end{array}$ & $\begin{array}{l}\text { Ortho- } \\
\text { phos- } \\
\text { phorus, } \\
\text { total } \\
\text { (as P) }\end{array}$ & $\begin{array}{l}\text { Organic } \\
\text { carbon, } \\
\text { total } \\
\text { (as C) }\end{array}$ \\
\hline & \multicolumn{6}{|c|}{ Big Spring (fig. 2) } \\
\hline $\begin{array}{l}10-05-89 \\
11-08-89 \\
12-05-89 \\
01-04-90 \\
01-22-90\end{array}$ & $\begin{array}{l}1145 \\
1500 \\
1400 \\
1300 \\
1125\end{array}$ & $\begin{array}{r}* 5.9 \\
* 5.7 \\
* 5.9 \\
* 6.3 \\
3.7\end{array}$ & $\begin{array}{c}*<0.10 \\
*<.10 \\
*<.10 \\
*<.10 \\
1.0\end{array}$ & $\begin{array}{c}* 0.50 \\
*<.10 \\
* \quad .20 \\
* \quad .20 \\
2.9\end{array}$ & $\begin{array}{c}* 0.50 \\
* \quad .50 \\
* .40 \\
* .40 \\
1.0\end{array}$ & $\begin{array}{c}1.0 \\
1.3 \\
<. .10 \\
1.3 \\
12\end{array}$ \\
\hline $\begin{array}{l}02-06-90 \\
02-08-90 \\
02-12-90 \\
03-05-90 \\
03-08-90\end{array}$ & $\begin{array}{l}1445 \\
0915 \\
1105 \\
1245 \\
1730\end{array}$ & $\begin{array}{l}5.0 \\
4.4 \\
3.0 \\
4.9 \\
3.8\end{array}$ & $\begin{array}{r}.20 \\
.40 \\
1.8 \\
<.10 \\
.80\end{array}$ & $\begin{array}{l}.70 \\
1.9 \\
2.9 \\
1.1 \\
5.3\end{array}$ & $\begin{array}{l}.80 \\
1.0 \\
1.0 \\
.80 \\
1.1\end{array}$ & $\begin{array}{c}4.5 \\
9.4 \\
14 \\
7.6 \\
19\end{array}$ \\
\hline $\begin{array}{l}03-09-90 \\
03-10-90 \\
03-12-90 \\
03-16-90 \\
04-02-90\end{array}$ & $\begin{array}{l}1630 \\
0815 \\
1200 \\
1330 \\
1340\end{array}$ & $\begin{array}{r}3.4 \\
3.8 \\
* 5.4 \\
8.1 \\
* 7.8\end{array}$ & $\begin{array}{r}1.6 \\
1.6 \\
* 1.4 \\
* 40 \\
.10\end{array}$ & $\begin{array}{r}6.4 \\
6.2 \\
* 5.0 \\
1.5 \\
* \quad .20\end{array}$ & $\begin{array}{r}1.2 \\
1.3 \\
* 1.1 \\
* \quad .70 \\
.30\end{array}$ & $\begin{array}{r}36 \\
33 \\
27 \\
9.7 \\
2.6\end{array}$ \\
\hline $\begin{array}{l}05-01-90 \\
06-06-90 \\
06-22-90 \\
06-23-90 \\
07-02-90\end{array}$ & $\begin{array}{l}1410 \\
1030 \\
1700 \\
0700 \\
1600\end{array}$ & $\begin{array}{r}6.0 \\
* 9.1 \\
10 \\
12 \\
14\end{array}$ & $\begin{array}{r}<.10 \\
*<.10 \\
<.10 \\
<.10 \\
<.10\end{array}$ & $\begin{array}{r}.20 \\
* \quad .20 \\
.60 \\
.50 \\
.20\end{array}$ & $\begin{array}{r}.30 \\
* \quad .30 \\
.30 \\
.40 \\
.20\end{array}$ & $\begin{array}{l}1.2 \\
1.9 \\
4.5 \\
3.4 \\
2.5\end{array}$ \\
\hline $\begin{array}{l}07-20-90 \\
08-01-90 \\
08-19-90 \\
08-20-90 \\
08-24-90\end{array}$ & $\begin{array}{l}1240 \\
1320 \\
1320 \\
1600 \\
0600\end{array}$ & $\begin{array}{l}10 \\
9.7 \\
9.4 \\
9.2 \\
8.9\end{array}$ & $\begin{array}{l}<.10 \\
<.10 \\
<.10 \\
<.10 \\
<.10\end{array}$ & $\begin{array}{l}.30 \\
.30 \\
.20 \\
.50 \\
.40\end{array}$ & $\begin{array}{l}.30 \\
.30 \\
-. \\
-. \\
.40\end{array}$ & $\begin{array}{r}2.1 \\
3.0 \\
-. \\
-. \\
2.3\end{array}$ \\
\hline $\begin{array}{l}08-24-90 \\
08-25-90 \\
08-26-90 \\
08-27-90 \\
08-28-90\end{array}$ & $\begin{array}{l}1600 \\
1000 \\
1215 \\
1045 \\
1040\end{array}$ & $\begin{array}{l}7.4 \\
9.8 \\
12 . \\
14 \\
16\end{array}$ & $\begin{array}{r}.20 \\
.20 \\
.10 \\
<.10 \\
<.10\end{array}$ & $\begin{array}{l}9.1 \\
2.9 \\
1.3 \\
.90 \\
.60\end{array}$ & $\begin{array}{l}.40 \\
.40 \\
.40 \\
.40 \\
.30\end{array}$ & $\begin{array}{l}5.5 \\
4.7 \\
4.6 \\
3.7 \\
-.\end{array}$ \\
\hline $\begin{array}{l}08-30-90 \\
09-05-90\end{array}$ & $\begin{array}{l}1200 \\
1430\end{array}$ & $\begin{array}{l}14 \\
12\end{array}$ & $\begin{array}{l}<.10 \\
<.10\end{array}$ & $\begin{array}{l}.40 \\
.20\end{array}$ & $\begin{array}{l}.20 \\
.20\end{array}$ & $\begin{array}{l}2.0 \\
2.9\end{array}$ \\
\hline
\end{tabular}

Dissolved constituents. 
Table 24. Selected pesticides at ground-water monitoring sites in the Big Spring basin, Clayton County, Iowa, water year 1990

[Total-recoverable constituents in micrograms per liter; <, less than the detection level indicated]

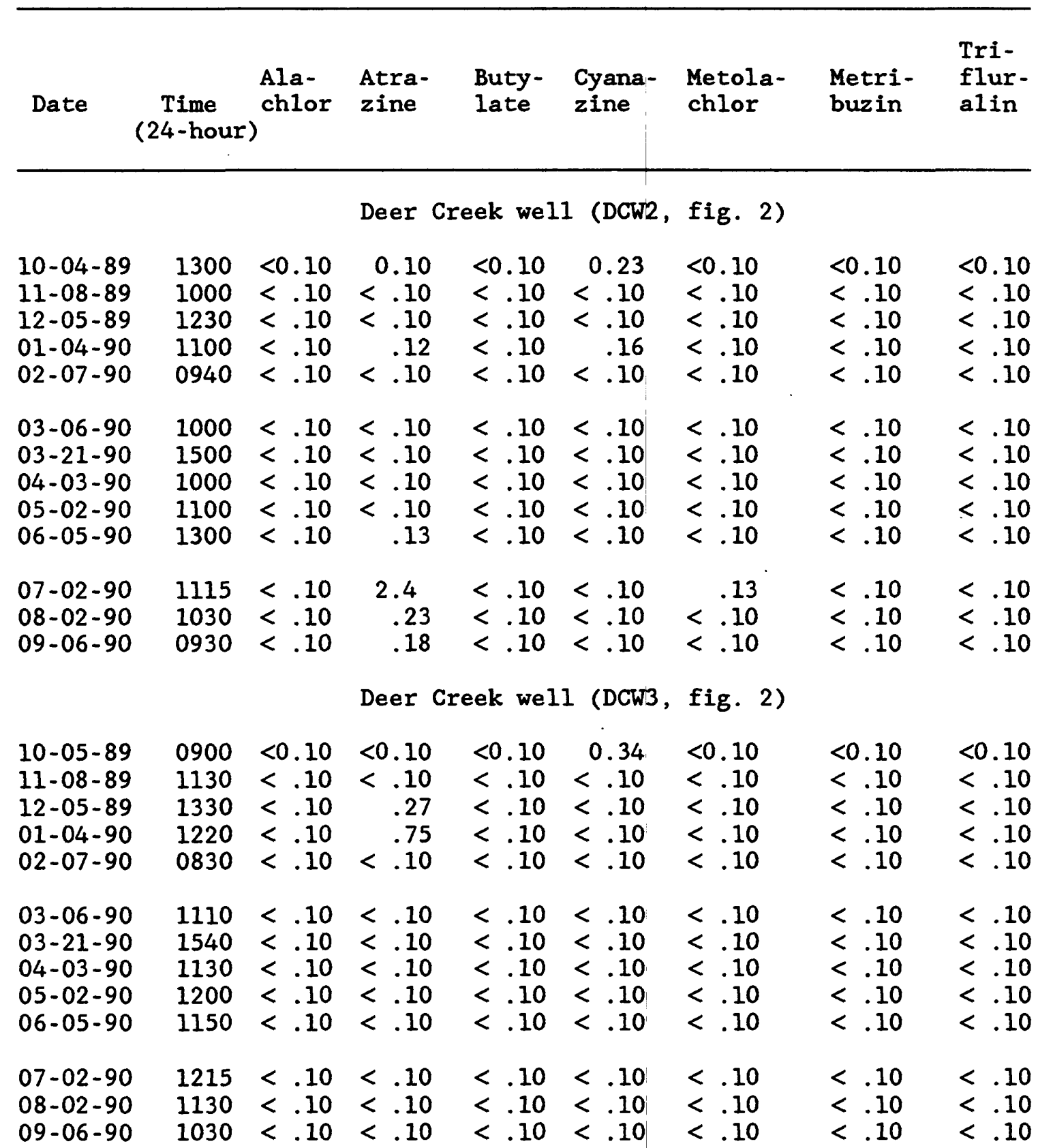


Table 24. Selected pesticides at ground-water monitoring sites in the Big Spring basin, Clayton County, Iowa, water year 1990--Continued

\begin{tabular}{|c|c|c|c|c|c|c|c|c|}
\hline Date & $\begin{array}{c}\text { Time } \\
\text { (24-hour) }\end{array}$ & $\begin{array}{l}\text { Ala- } \\
\text { chlor }\end{array}$ & $\begin{array}{l}\text { Atra- } \\
\text { zine }\end{array}$ & $\begin{array}{l}\text { Buty- } \\
\text { late }\end{array}$ & $\begin{array}{l}\text { Cyana- } \\
\text { zine }\end{array}$ & $\begin{array}{l}\text { Metola- } \\
\text { chlor }\end{array}$ & $\begin{array}{l}\text { Metri- } \\
\text { buzin }\end{array}$ & $\begin{array}{l}\text { Tri- } \\
\text { flur- } \\
\text { alin }\end{array}$ \\
\hline
\end{tabular}

Deer Creek lysimeter (DCLA, fig. 2)

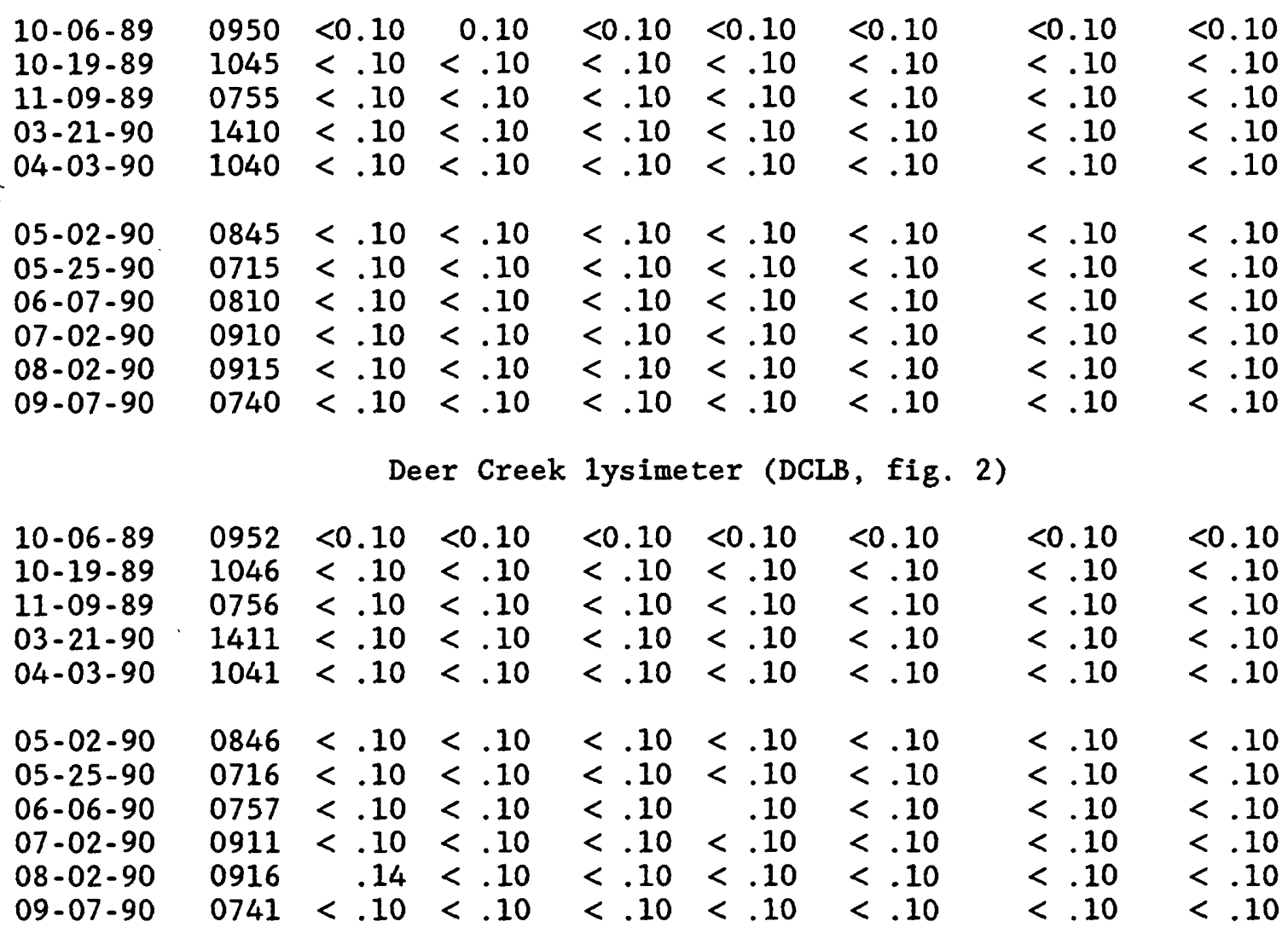


Table 24. Selected pesticides at ground-water monitoring sites in the Big Spring basin, Clayton County, Iowa, water year 1990--Continued

\begin{tabular}{|c|c|c|c|c|c|c|c|c|}
\hline Date & $\begin{array}{c}\text { Time } \\
\text { (24-hour) }\end{array}$ & $\begin{array}{l}\text { Ala- } \\
\text { chlor }\end{array}$ & $\begin{array}{l}\text { Atra- } \\
\text { zine }\end{array}$ & $\begin{array}{l}\text { Buty- } \\
\text { late }\end{array}$ & $\begin{array}{l}\text { Cyana- } \\
\text { zine }\end{array}$ & $\begin{array}{l}\text { Metola- } \\
\text { chlor }\end{array}$ & $\begin{array}{l}\text { Metri- } \\
\text { buzin }\end{array}$ & $\begin{array}{l}\text { Tri- } \\
\text { flur- } \\
\text { alin }\end{array}$ \\
\hline
\end{tabular}

Deer Creek lysimeter (DCLC, fig. 2)

\begin{tabular}{|c|c|c|c|c|c|c|c|c|}
\hline $\begin{array}{l}10-06-89 \\
10-19-89 \\
11-09-89 \\
03-21-90 \\
04-03-90\end{array}$ & $\begin{array}{l}0954 \\
1047 \\
0757 \\
1412 \\
1042\end{array}$ & $\begin{array}{l}<0.10 \\
<.10 \\
<.10 \\
<.10 \\
<.10\end{array}$ & $\begin{array}{r}0.57 \\
.59 \\
.31 \\
.14 \\
<.10\end{array}$ & $\begin{array}{l}<0.10 \\
<.10 \\
<.10 \\
<.10 \\
<.10\end{array}$ & $\begin{array}{l}<0.10 \\
<.10 \\
<.10 \\
<.10 \\
<.10\end{array}$ & $\begin{array}{l}<0.10 \\
<.10 \\
<.10 \\
<.10 \\
<.10\end{array}$ & $\begin{array}{l}<0.10 \\
<.10 \\
<.10 \\
<.10 \\
<.10\end{array}$ & $\begin{array}{l}<0.10 \\
<.10 \\
<.10 \\
<.10 \\
<.10\end{array}$ \\
\hline $\begin{array}{l}05-02-90 \\
05-25-90 \\
06-06-90 \\
07-02-90 \\
08-02-90\end{array}$ & $\begin{array}{l}0847 \\
0717 \\
0758 \\
0912 \\
0917\end{array}$ & $\begin{array}{l}<.10 \\
<.10 \\
<.10 \\
<.10 \\
<.10\end{array}$ & $\begin{array}{r}.10 \\
.10 \\
<.10 \\
.12 \\
.16\end{array}$ & $\begin{array}{l}<.10 \\
<.10 \\
<.10 \\
<.10 \\
<.10\end{array}$ & $\begin{array}{l}<.10 \\
<.10 \\
<.10 \\
<.10 \\
<.10\end{array}$ & $\begin{array}{l}<.10 \\
<.10 \\
<.10 \\
<.10 \\
<.10\end{array}$ & $\begin{array}{l}<.10 \\
<.10 \\
<.10 \\
<.10 \\
<.10\end{array}$ & $\begin{array}{l}<.10 \\
<.10 \\
<.10 \\
<.10 \\
<.10\end{array}$ \\
\hline & \multicolumn{8}{|c|}{ Deer Creek } \\
\hline $\begin{array}{l}11-09-89 \\
03-21-90 \\
04-03-90 \\
05-02-90 \\
05-25-90\end{array}$ & $\begin{array}{l}0758 \\
1413 \\
1043 \\
0848 \\
0718\end{array}$ & $\begin{array}{l}<0.10 \\
<.10 \\
<.10 \\
<.10 \\
<.10\end{array}$ & $\begin{array}{r}0.35 \\
.16 \\
.12 \\
.14 \\
1.0\end{array}$ & $\begin{array}{l}<0.10 \\
<.10 \\
<.10 \\
<.10 \\
<.10\end{array}$ & $\begin{array}{l}<0.10 \\
<.10 \\
<.10 \\
<.10 \\
<.10\end{array}$ & $\begin{array}{l}<0.10 \\
<.10 \\
<.10 \\
<.10 \\
.34\end{array}$ & $\begin{array}{l}<0.10 \\
<.10 \\
<.10 \\
<.10 \\
<.10\end{array}$ & $\begin{array}{l}<0.10 \\
<.10 \\
<.10 \\
<.10 \\
<.10\end{array}$ \\
\hline $\begin{array}{l}06-06-90 \\
07-02-90 \\
08-02-90 \\
09-07-90\end{array}$ & $\begin{array}{l}0759 \\
0913 \\
0918 \\
0743\end{array}$ & $\begin{array}{l}<.10 \\
<.10 \\
<.10 \\
<.10\end{array}$ & $\begin{array}{l}1.3 \\
3.6 \\
1.8 \\
3.1\end{array}$ & $\begin{array}{l}<.10 \\
<.10 \\
<.10 \\
<.10\end{array}$ & $\begin{array}{l}<.10 \\
<.10 \\
<.10 \\
<.10\end{array}$ & $\begin{array}{l}.55 \\
.83 \\
.33 \\
.27\end{array}$ & $\begin{array}{l}<.10 \\
<.10 \\
<.10 \\
<.10\end{array}$ & $\begin{array}{l}<.10 \\
<.10 \\
<.10 \\
<.10\end{array}$ \\
\hline
\end{tabular}

Deer Creek tile 2 (DCT2, fig. 2)

\begin{tabular}{|c|c|c|c|c|c|c|c|c|}
\hline $\begin{array}{l}11-08-89 \\
12-05-89 \\
01-04-90 \\
02-07-90 \\
03-06-90\end{array}$ & $\begin{array}{l}0915 \\
0900 \\
0820 \\
1030 \\
0800\end{array}$ & $\begin{array}{l}<0.10 \\
<.10 \\
<.10 \\
<.10 \\
<.10\end{array}$ & $\begin{array}{r}0.14 \\
.10 \\
<.10 \\
.10 \\
<.10\end{array}$ & $\begin{array}{l}<0.10 \\
<.10 \\
<.10 \\
<.10 \\
<.10\end{array}$ & $\begin{array}{l}<0.10 \\
<.10 \\
<.10 \\
<.10 \\
<.10\end{array}$ & $\begin{array}{l}<0.10 \\
<.10 \\
<.10 \\
<.10 \\
<.10\end{array}$ & $\begin{array}{l}<0.10 \\
<.10 \\
<.10 \\
<.10 \\
<.10\end{array}$ & $\begin{array}{l}<0.10 \\
<.10 \\
<.10 \\
<.10 \\
<.10\end{array}$ \\
\hline $\begin{array}{l}04-03-90 \\
05-02-90 \\
06-05-90 \\
07-02-90 \\
08-02-90\end{array}$ & $\begin{array}{l}0815 \\
0815 \\
1100 \\
0940 \\
0815\end{array}$ & $\begin{array}{l}<.10 \\
<.10 \\
<.10 \\
<.10 \\
<.10\end{array}$ & $\begin{array}{l}.17 \\
.10 \\
.37 \\
.48 \\
.42\end{array}$ & $\begin{array}{l}<.10 \\
<.10 \\
<.10 \\
<.10 \\
<.10\end{array}$ & $\begin{array}{l}<.10 \\
<.10 \\
<.10 \\
<.10 \\
<.10\end{array}$ & $\begin{array}{l}<.10 \\
<.10 \\
.85 \\
.16 \\
<.10\end{array}$ & $\begin{array}{l}<.10 \\
<.10 \\
<.10 \\
<.10 \\
<.10\end{array}$ & $\begin{array}{l}<.10 \\
<.10 \\
.23 \\
<.10 \\
<.10\end{array}$ \\
\hline $09-06-90$ & 0730 & $<.10$ & $<.10$ & $<.10$ & $<.10$ & $<.10$ & $<.10$ & $<.10$ \\
\hline
\end{tabular}


Table 24. Selected pesticides at ground-water monitoring sites in the Big Spring basin, Clayton County, Iowa, water year 1990--Continued

\begin{tabular}{|c|c|c|c|c|c|c|c|c|}
\hline Date & $\begin{array}{c}\text { Time } \\
\text { (24-hour) }\end{array}$ & $\begin{array}{l}\text { Ala: } \\
\text { chlor }\end{array}$ & $\begin{array}{l}\text { Atra- } \\
\text { zine }\end{array}$ & $\begin{array}{l}\text { Buty- } \\
\text { late }\end{array}$ & $\begin{array}{l}\text { Cyana- } \\
\text { zine }\end{array}$ & $\begin{array}{l}\text { Metola- } \\
\text { chlor }\end{array}$ & $\begin{array}{l}\text { Metri- } \\
\text { buzin }\end{array}$ & $\begin{array}{l}\text { Tri- } \\
\text { flur- } \\
\text { alin }\end{array}$ \\
\hline
\end{tabular}

\begin{tabular}{|c|c|c|c|c|c|c|c|c|}
\hline & & & Big & ing & ig. 2) & & & \\
\hline $\begin{array}{l}10-05-89 \\
11-08-89 \\
12-05-89 \\
01-04-90 \\
01-22-90\end{array}$ & $\begin{array}{l}1145 \\
1500 \\
1400 \\
1300 \\
1125\end{array}$ & $\begin{array}{l}<0.10 \\
<.10 \\
<.10 \\
<.10 \\
.122\end{array}$ & $\begin{array}{r}0.38 \\
.22 \\
.19 \\
.14 \\
1.4\end{array}$ & $\begin{array}{l}<0.10 \\
<.10 \\
<.10 \\
<.10 \\
<.10\end{array}$ & $\begin{array}{r}0.26 \\
<\quad .10 \\
<.10 \\
<.10 \\
.24\end{array}$ & $\begin{array}{l}<0.10 \\
<.10 \\
<.10 \\
<.10 \\
<.10\end{array}$ & $\begin{array}{l}<0.10 \\
<.10 \\
<.10 \\
<.10 \\
<.10\end{array}$ & $\begin{array}{l}<0.10 \\
<.10 \\
<.10 \\
<.10 \\
<.10\end{array}$ \\
\hline $\begin{array}{l}02-06-90 \\
02-07-90 \\
02-12-90 \\
03-05-90 \\
03-08-90\end{array}$ & $\begin{array}{l}1445 \\
1545 \\
1105 \\
1245 \\
1730\end{array}$ & $\begin{array}{l}<.10 \\
<.10 \\
<.10 \\
<.10 \\
<.10\end{array}$ & $\begin{array}{l}.69 \\
1.2 \\
2.7 \\
.68 \\
1.0\end{array}$ & $\begin{array}{l}<.10 \\
<.10 \\
<.10 \\
<.10 \\
<.10\end{array}$ & $\begin{array}{r}<.10 \\
<.10 \\
.16 \\
<.10 \\
<.10\end{array}$ & $\begin{array}{c}<.10 \\
<.10 \\
2.1 \\
<.10 \\
<.10\end{array}$ & $\begin{array}{l}<.10 \\
<.10 \\
<.10 \\
<.10 \\
<.10\end{array}$ & $\begin{array}{l}<.10 \\
<.10 \\
<.10 \\
<.10 \\
<.10\end{array}$ \\
\hline $\begin{array}{l}03-09-90 \\
03-10-90 \\
03-12-90 \\
03-16-90 \\
04-02-90\end{array}$ & $\begin{array}{l}1630 \\
0815 \\
1200 \\
1330 \\
1340\end{array}$ & $\begin{array}{r}<.10 \\
.13 \\
.13 \\
<.10 \\
<.10\end{array}$ & $\begin{array}{l}3.0 \\
2.7 \\
4.5 \\
2.1 \\
.37\end{array}$ & $\begin{array}{l}<.10 \\
<.10 \\
<.10 \\
<.10 \\
<.10\end{array}$ & $\begin{array}{r}.22 \\
.19 \\
.22 \\
.29 \\
<.10\end{array}$ & $\begin{array}{l}<.10 \\
<.10 \\
<.10 \\
<.10 \\
<.10\end{array}$ & $\begin{array}{l}<.10 \\
<.10 \\
<.10 \\
<.10 \\
<.10\end{array}$ & $\begin{array}{l}<.10 \\
<.10 \\
<.10 \\
<.10 \\
<.10\end{array}$ \\
\hline $\begin{array}{l}05-01-90 \\
06-06-90 \\
06-22-90 \\
06-23-90 \\
07-02-90\end{array}$ & $\begin{array}{l}1410 \\
1030 \\
1700 \\
0700 \\
1600\end{array}$ & $\begin{array}{r}<.10 \\
.77 \\
.25 \\
.39 \\
.12\end{array}$ & $\begin{array}{l}.19 \\
1.6 \\
2.3 \\
1.4 \\
1.8\end{array}$ & $\begin{array}{l}<.10 \\
<.10 \\
<.10 \\
<.10 \\
<.10\end{array}$ & $\begin{array}{r}<.10 \\
.47 \\
.40 \\
.45 \\
.21\end{array}$ & $\begin{array}{l}<.10 \\
<.10 \\
\\
<.18 \\
<.10 \\
<.10\end{array}$ & $\begin{array}{l}<.10 \\
<.10 \\
<.10 \\
<.10 \\
<.10\end{array}$ & $\begin{array}{l}<.10 \\
<.10 \\
<.10 \\
<.10 \\
<.10\end{array}$ \\
\hline $\begin{array}{l}07-20-90 \\
08-01-90 \\
08-19-90 \\
08-20-90 \\
08-24-90\end{array}$ & $\begin{array}{l}1240 \\
1320 \\
1520 \\
1600 \\
0600\end{array}$ & $\begin{array}{l}<.10 \\
<.10 \\
<.10 \\
<.10 \\
<.10\end{array}$ & $\begin{array}{l}.98 \\
.64 \\
.61 \\
.49 \\
.44\end{array}$ & $\begin{array}{l}<.10 \\
<.10 \\
<.10 \\
<.10 \\
<.10\end{array}$ & $\begin{array}{l}<.10 \\
<.10 \\
<.10 \\
<.10 \\
<.10\end{array}$ & $\begin{array}{l}<.10 \\
<.10 \\
<.10 \\
<.10 \\
<.10\end{array}$ & $\begin{array}{l}<.10 \\
<.10 \\
<.10 \\
<.10 \\
<.10\end{array}$ & $\begin{array}{l}<.10 \\
<.10 \\
<.10 \\
<.10 \\
<.10\end{array}$ \\
\hline $\begin{array}{l}08-24-90 \\
08-25-90 \\
08-26-90 \\
08-27-90 \\
08-28-90\end{array}$ & $\begin{array}{l}1600 \\
0600 \\
1215 \\
1045 \\
1040\end{array}$ & $\begin{array}{l}<.10 \\
<.10 \\
<.10 \\
<.10 \\
<.10\end{array}$ & $\begin{array}{l}.40 \\
.89 \\
.93 \\
.86 \\
1.2\end{array}$ & $\begin{array}{l}<.10 \\
<.10 \\
<.10 \\
<.10 \\
<.10\end{array}$ & $\begin{array}{r}<.10 \\
.12 \\
.15 \\
.12 \\
<.10\end{array}$ & $\begin{array}{l}<.10 \\
<.10 \\
<.10 \\
<.10 \\
<.10\end{array}$ & $\begin{array}{l}<.10 \\
<.10 \\
<.10 \\
<.10 \\
<.10\end{array}$ & $\begin{array}{l}<.10 \\
<.10 \\
<.10 \\
<.10 \\
<.10\end{array}$ \\
\hline $\begin{array}{l}08-30-90 \\
09-05-90\end{array}$ & $\begin{array}{l}1200 \\
1430\end{array}$ & $\begin{array}{l}<.10 \\
<.10\end{array}$ & $\begin{array}{l}.43 \\
.32\end{array}$ & $\begin{array}{l}<.10 \\
<.10\end{array}$ & $\begin{array}{l}<.10 \\
<.10\end{array}$ & $\begin{array}{l}<.10 \\
<.10\end{array}$ & $\begin{array}{l}<.10 \\
<.10\end{array}$ & $\begin{array}{l}<.10 \\
<.10\end{array}$ \\
\hline
\end{tabular}


Table 25. Daily mean water levels in the Galena aquifer, Clayton County, Iowa, water year 1990

[Water levels in feet below land surface; ---, data not available to calculate mean values]

\begin{tabular}{|c|c|c|c|c|c|c|c|c|c|c|c|c|}
\hline Day & Oct. & Nov. & Dec. & Jan. & Fob. & Mar. & Apr. & May & June & July & Aug. & pt \\
\hline \multicolumn{13}{|c|}{ Well BS1-B (fig. 2) } \\
\hline $\begin{array}{l}1 \\
2 \\
3 \\
4 \\
5\end{array}$ & $\begin{array}{l}--- \\
--- \\
--- \\
---\end{array}$ & $\begin{array}{l}--- \\
--- \\
--- \\
--\end{array}$ & $\begin{array}{l}--- \\
--- \\
--- \\
---\end{array}$ & $\begin{array}{r}--- \\
--- \\
10.34 \\
10.33\end{array}$ & $\begin{array}{r}9.95 \\
9.94 \\
9.96 \\
10.02 \\
9.80\end{array}$ & $\begin{array}{l}9.58 \\
8.87 \\
8.71 \\
8.87 \\
9.12\end{array}$ & $\begin{array}{l}7.50 \\
7.57 \\
7.60 \\
7.62 \\
7.71\end{array}$ & $\begin{array}{l}7.80 \\
7.82 \\
7.87 \\
7.88 \\
7.92\end{array}$ & $\begin{array}{l}6.55 \\
6.48 \\
6.28 \\
6.31 \\
6.30\end{array}$ & $\begin{array}{l}6.09 \\
6.21 \\
6.31 \\
6.46 \\
6.59\end{array}$ & $\begin{array}{l}6.58 \\
6.77 \\
6.93 \\
6.91 \\
7.00\end{array}$ & $\begin{array}{l}--- \\
--- \\
-- \\
-- \\
--\end{array}$ \\
\hline $\begin{array}{r}6 \\
7 \\
8 \\
9 \\
10\end{array}$ & $\begin{array}{l}9.69 \\
9.80 \\
9.86 \\
9.88 \\
9.91\end{array}$ & $\begin{array}{r}--- \\
--- \\
9.52 \\
9.56 \\
9.65\end{array}$ & $\begin{array}{l}--- \\
--- \\
--- \\
---\end{array}$ & $\begin{array}{r}10.32 \\
10.30 \\
10.28 \\
9.87 \\
9.42\end{array}$ & $\begin{array}{l}8.69 \\
8.56 \\
8.12 \\
7.78 \\
8.11\end{array}$ & $\begin{array}{l}9.30 \\
9.32 \\
7.68 \\
6.10 \\
6.20\end{array}$ & $\begin{array}{l}7.78 \\
7.86 \\
7.90 \\
7.94 \\
7.85\end{array}$ & $\begin{array}{l}7.98 \\
8.02 \\
8.11 \\
8.08 \\
7.92\end{array}$ & $\begin{array}{l}6.38 \\
6.44 \\
6.50 \\
6.61 \\
6.73\end{array}$ & $\begin{array}{l}6.68 \\
6.73 \\
6.80 \\
6.91 \\
6.99\end{array}$ & $\begin{array}{l}6.99 \\
7.08 \\
7.25 \\
7.38 \\
7.49\end{array}$ & $\begin{array}{l}--- \\
--- \\
--- \\
---\end{array}$ \\
\hline $\begin{array}{l}11 \\
12 \\
13 \\
14 \\
15\end{array}$ & $\begin{array}{r}9.91 \\
9.98 \\
10.00 \\
10.07 \\
10.00\end{array}$ & $\begin{array}{l}9.67 \\
9.67 \\
9.65 \\
9.67 \\
9.68\end{array}$ & $\begin{array}{l}--- \\
--- \\
--- \\
---\end{array}$ & $\begin{array}{r}9.39 \\
9.93 \\
10.04 \\
10.06 \\
10.13\end{array}$ & $\begin{array}{l}8.55 \\
8.70 \\
8.38 \\
8.75 \\
9.03\end{array}$ & $\begin{array}{l}5.57 \\
5.40 \\
5.16 \\
4.46 \\
4.01\end{array}$ & $\begin{array}{l}7.78 \\
7.79 \\
7.82 \\
7.81 \\
7.82\end{array}$ & $\begin{array}{l}7.89 \\
7.79 \\
7.78 \\
7.79 \\
7.84\end{array}$ & $\begin{array}{l}6.80 \\
6.79 \\
6.63 \\
6.27 \\
6.16\end{array}$ & $\begin{array}{l}7.03 \\
7.09 \\
7.10 \\
7.13 \\
7.21\end{array}$ & $\begin{array}{l}7.57 \\
7.58 \\
7.77 \\
7.92 \\
8.00\end{array}$ & $\begin{array}{l}--- \\
--- \\
--- \\
---\end{array}$ \\
\hline $\begin{array}{l}16 \\
17 \\
18 \\
19 \\
20\end{array}$ & $\begin{array}{l}9.70 \\
9.54 \\
9.60 \\
9.59 \\
9.62\end{array}$ & \begin{tabular}{r}
9.71 \\
9.77 \\
\hdashline-- \\
---
\end{tabular} & $\begin{array}{r}--- \\
--- \\
--- \\
10.03\end{array}$ & $\begin{array}{c}10.02 \\
9.10 \\
9.16 \\
9.54 \\
9.67\end{array}$ & $\begin{array}{l}9.17 \\
9.30 \\
9.32 \\
9.44 \\
9.49\end{array}$ & $\begin{array}{l}3.86 \\
4.53 \\
5.15 \\
5.62 \\
5.93\end{array}$ & $\begin{array}{l}7.83 \\
7.88 \\
7.90 \\
7.88 \\
7.82\end{array}$ & $\begin{array}{l}7.84 \\
7.90 \\
7.96 \\
7.43 \\
6.22\end{array}$ & $\begin{array}{l}6.07 \\
5.96 \\
5.86 \\
5.77 \\
5.82\end{array}$ & $\begin{array}{l}7.33 \\
7.43 \\
7.43 \\
7.28 \\
7.06\end{array}$ & $\begin{array}{l}8.09 \\
8.05 \\
7.94 \\
7.61 \\
7.11\end{array}$ & $\begin{array}{r}--- \\
--13 \\
6.13 \\
6.21 \\
6.28\end{array}$ \\
\hline $\begin{array}{l}21 \\
22 \\
23 \\
24 \\
25\end{array}$ & $\begin{array}{c}9.71 \\
--- \\
--- \\
---\end{array}$ & $\begin{array}{l}--- \\
--\overline{-} \\
--- \\
---\end{array}$ & $\begin{array}{r}10.04 \\
10.14 \\
10.09 \\
--- \\
--\end{array}$ & $\begin{array}{l}9.77 \\
9.84 \\
9.84 \\
9.87 \\
9.82\end{array}$ & $\begin{array}{l}9.50 \\
9.45 \\
9.30 \\
9.36 \\
9.49\end{array}$ & $\begin{array}{l}6.14 \\
6.39 \\
6.59 \\
6.72 \\
6.82\end{array}$ & $\begin{array}{l}7.75 \\
7.70 \\
7.68 \\
7.71 \\
7.77\end{array}$ & $\begin{array}{l}5.58 \\
5.46 \\
5.55 \\
5.70 \\
5.80\end{array}$ & $\begin{array}{l}5.94 \\
5.54 \\
5.05 \\
5.06 \\
5.24\end{array}$ & $\begin{array}{l}7.01 \\
7.05 \\
7.18 \\
7.30 \\
7.41\end{array}$ & $\begin{array}{l}6.22 \\
6.04 \\
6.29 \\
6.35 \\
-.-\end{array}$ & $\begin{array}{l}6.32 \\
6.41 \\
6.45 \\
6.50 \\
6.53\end{array}$ \\
\hline $\begin{array}{l}26 \\
27 \\
28 \\
29 \\
30 \\
31\end{array}$ & $\begin{array}{l}--- \\
--- \\
--- \\
---\end{array}$ & $\begin{array}{l}--- \\
--- \\
--- \\
---\end{array}$ & $\begin{array}{l}--- \\
--- \\
--- \\
--- \\
---\end{array}$ & $\begin{array}{r}9.86 \\
9.87 \\
9.96 \\
9.92 \\
9.94 \\
10.01\end{array}$ & $\begin{array}{l}9.49 \\
9.60 \\
9.65\end{array}$ & $\begin{array}{l}6.98 \\
7.07 \\
7.16 \\
7.26 \\
7.35 \\
7.45\end{array}$ & $\begin{array}{l}7.77 \\
7.80 \\
7.82 \\
7.82 \\
7.81\end{array}$ & $\begin{array}{l}5.89 \\
6.00 \\
6.10 \\
6.15 \\
6.31 \\
6.45\end{array}$ & $\begin{array}{l}5.36 \\
5.52 \\
5.69 \\
5.84 \\
5.95\end{array}$ & $\begin{array}{l}7.49 \\
7.29 \\
6.37 \\
6.10 \\
6.15 \\
6.35\end{array}$ & $\begin{array}{l}--- \\
--- \\
-- \\
-- \\
--\end{array}$ & $\begin{array}{l}6.62 \\
6.64 \\
6.73 \\
6.77 \\
6.79\end{array}$ \\
\hline \multicolumn{13}{|c|}{ We1l BS2-E (fig. 2) } \\
\hline $\begin{array}{l}1 \\
2 \\
3 \\
4 \\
5\end{array}$ & $\begin{array}{l}154.54 \\
154.60 \\
154.59 \\
154.58 \\
154.56\end{array}$ & $\begin{array}{l}154.42 \\
154.44 \\
154.43 \\
154.46 \\
154.49\end{array}$ & $\begin{array}{l}154.58 \\
154.62 \\
154.60 \\
154.60 \\
154.62\end{array}$ & $\begin{array}{l}154.75 \\
154.74 \\
154.79 \\
154.84 \\
154.83\end{array}$ & $\begin{array}{l}154.70 \\
154.71 \\
154.72 \\
154.72 \\
153.61\end{array}$ & $\begin{array}{l}154.12 \\
153.79 \\
154.17 \\
154.26 \\
154.40\end{array}$ & $\begin{array}{l}154.43 \\
154.39 \\
154.37 \\
154.37 \\
154.38\end{array}$ & $\begin{array}{l}154.34 \\
154.36 \\
154.37 \\
154.35 \\
154.35\end{array}$ & $\begin{array}{l}153.98 \\
153.71 \\
153.70 \\
153.82 \\
153.86\end{array}$ & $\begin{array}{l}153.43 \\
153.51 \\
153.55 \\
153.63 \\
153.67\end{array}$ & $\begin{array}{l}153.95 \\
153.92 \\
153.93 \\
153.69 \\
153.85\end{array}$ & $\begin{array}{l}--- \\
--- \\
--- \\
---\end{array}$ \\
\hline $\begin{array}{r}6 \\
7 \\
8 \\
9 \\
10\end{array}$ & $\begin{array}{l}154.55 \\
154.55 \\
154.56 \\
154.56 \\
154.58\end{array}$ & $\begin{array}{l}154.50 \\
154.47 \\
154.48 \\
154.52 \\
154.51\end{array}$ & $\begin{array}{l}154.64 \\
154.64 \\
154.61 \\
154.61 \\
154.64\end{array}$ & $\begin{array}{l}154.83 \\
154.83 \\
154.80 \\
153.90 \\
154.42\end{array}$ & $\begin{array}{l}153.61 \\
153.39 \\
151.96 \\
153.69 \\
154.20\end{array}$ & $\begin{array}{r}154.47 \\
154.49 \\
--- \\
--.\end{array}$ & $\begin{array}{l}154.39 \\
154.40 \\
154.40 \\
154.40 \\
154.36\end{array}$ & $\begin{array}{l}154.35 \\
154.35 \\
154.38 \\
154.31 \\
154.28\end{array}$ & $\begin{array}{l}153.99 \\
154.03 \\
154.03 \\
154.04 \\
154.06\end{array}$ & $\begin{array}{l}153.69 \\
153.70 \\
153.72 \\
153.76 \\
153.77\end{array}$ & $\begin{array}{l}153.92 \\
153.94 \\
153.95 \\
153.96 \\
153.96\end{array}$ & $\begin{array}{r}153.49 \\
-=- \\
--- \\
---\end{array}$ \\
\hline $\begin{array}{l}11 \\
12 \\
13 \\
14 \\
15\end{array}$ & $\begin{array}{l}154.58 \\
154.62 \\
154.60 \\
154.59 \\
154.59\end{array}$ & $\begin{array}{l}154.55 \\
154.51 \\
154.54 \\
154.53 \\
154.55\end{array}$ & $\begin{array}{l}154.64 \\
154.64 \\
154.66 \\
154.69 \\
154.69\end{array}$ & $\begin{array}{l}154.65 \\
154.76 \\
154.75 \\
154.75 \\
154.70\end{array}$ & $\begin{array}{l}154.37 \\
154.10 \\
154.23 \\
154.45 \\
154.48\end{array}$ & $\begin{array}{r}--- \\
153.40 \\
151.60 \\
152.38\end{array}$ & $\begin{array}{l}154.35 \\
154.34 \\
154.34 \\
154.34 \\
154.35\end{array}$ & $\begin{array}{l}154.29 \\
154.29 \\
154.33 \\
154.32 \\
154.32\end{array}$ & $\begin{array}{l}154.06 \\
154.06 \\
153.91 \\
153.95 \\
154.02\end{array}$ & $\begin{array}{l}153.78 \\
153.86 \\
153.86 \\
153.87 \\
153.89\end{array}$ & $\begin{array}{l}153.98 \\
153.99 \\
154.07 \\
154.21 \\
154.24\end{array}$ & $\begin{array}{l}--- \\
--- \\
--- \\
---\end{array}$ \\
\hline $\begin{array}{l}16 \\
17 \\
18 \\
19 \\
20\end{array}$ & $\begin{array}{l}154.41 \\
154.48 \\
154.52 \\
154.49 \\
154.50\end{array}$ & $\begin{array}{l}154.55 \\
154.59 \\
154.59 \\
154.57 \\
154.60\end{array}$ & $\begin{array}{l}154.70 \\
154.71 \\
154.73 \\
154.72 \\
154.73\end{array}$ & $\begin{array}{l}154.54 \\
153.11 \\
154.53 \\
154.60 \\
154.66\end{array}$ & $\begin{array}{l}154.56 \\
154.58 \\
154.58 \\
154.64 \\
154.63\end{array}$ & $\begin{array}{l}153.68 \\
154.18 \\
154.60 \\
154.66 \\
154.64\end{array}$ & $\begin{array}{l}154.36 \\
154.37 \\
154.37 \\
154.36 \\
154.34\end{array}$ & $\begin{array}{l}154.30 \\
154.31 \\
154.32 \\
153.92 \\
153.58\end{array}$ & $\begin{array}{l}153.76 \\
153.54 \\
153.56 \\
153.60 \\
153.64\end{array}$ & $\begin{array}{l}153.99 \\
154.01 \\
153.87 \\
153.51 \\
153.61\end{array}$ & $\begin{array}{l}154.24 \\
154.21 \\
154.20 \\
153.89 \\
154.00\end{array}$ & $\begin{array}{l}--- \\
--- \\
---\end{array}$ \\
\hline $\begin{array}{l}21 \\
22 \\
23 \\
24 \\
25\end{array}$ & $\begin{array}{l}154.55 \\
154.56 \\
154.56 \\
154.57 \\
154.57\end{array}$ & $\begin{array}{l}154.57 \\
154.58 \\
154.57 \\
154.56 \\
154.59\end{array}$ & $\begin{array}{l}154.75 \\
154.75 \\
154.73 \\
154.73 \\
154.74\end{array}$ & $\begin{array}{l}154.70 \\
154.72 \\
154.71 \\
154.76 \\
154.74\end{array}$ & $\begin{array}{l}154.60 \\
154.53 \\
154.49 \\
154.63 \\
154.62\end{array}$ & $\begin{array}{l}154.62 \\
154.56 \\
154.50 \\
154.50 \\
154.50\end{array}$ & $\begin{array}{l}154.32 \\
154.30 \\
154.27 \\
154.27 \\
154.29\end{array}$ & $\begin{array}{l}153.64 \\
153.69 \\
153.75 \\
153.80 \\
153.83\end{array}$ & $\begin{array}{l}153.68 \\
152.22 \\
152.40 \\
152.83 \\
153.03\end{array}$ & $\begin{array}{l}153.74 \\
153.80 \\
153.87 \\
153.98 \\
154.00\end{array}$ & $\begin{array}{l}154.04 \\
154.09 \\
154.12 \\
154.01 \\
142.54\end{array}$ & $\begin{array}{l}--- \\
--- \\
--- \\
--\end{array}$ \\
\hline $\begin{array}{l}26 \\
27 \\
28 \\
29 \\
30 \\
31\end{array}$ & $\begin{array}{l}154.56 \\
154.56 \\
154.55 \\
154.54 \\
154.47 \\
154.40\end{array}$ & $\begin{array}{l}154.59 \\
154.57 \\
154.60 \\
154.58 \\
154.59\end{array}$ & $\begin{array}{l}154.77 \\
154.76 \\
154.76 \\
154.76 \\
154.75 \\
154.75\end{array}$ & $\begin{array}{l}154.74 \\
154.77 \\
154.75 \\
154.72 \\
154.76 \\
154.73\end{array}$ & $\begin{array}{l}154.59 \\
154.64 \\
154.63\end{array}$ & $\begin{array}{l}154.45 \\
154.44 \\
154.45 \\
154.45 \\
154.44 \\
154.44\end{array}$ & $\begin{array}{l}154.29 \\
154.29 \\
154.29 \\
154.31 \\
154.34\end{array}$ & $\begin{array}{l}153.87 \\
153.90 \\
153.90 \\
153.91 \\
153.96 \\
153.98\end{array}$ & $\begin{array}{l}152.50 \\
153.04 \\
153.21 \\
153.29 \\
153.34\end{array}$ & $\begin{array}{l}154.01 \\
153.74 \\
153.73 \\
153.77 \\
153.86 \\
153.95\end{array}$ & $\begin{array}{l}--- \\
--- \\
-- \\
--- \\
---\end{array}$ & $\begin{array}{l}--- \\
--- \\
---\end{array}$ \\
\hline
\end{tabular}


Table 25. Daily mean water levels in the Galena aquifer, Clayton County, Iowa, water year 1990--Continued

\begin{tabular}{|c|c|c|c|c|c|c|c|c|c|c|c|c|}
\hline Day & Oct. & Nov. & Dec. & $\operatorname{Jan}$ & Feb. & Mar. & Apr. & May & June & July & Aus. & Sept. \\
\hline & & & & & 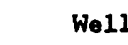 & $S 3-A$ & 8. 2) & & & & & \\
\hline $\begin{array}{l}1 \\
2 \\
3 \\
4 \\
5\end{array}$ & $\begin{array}{l}-- \\
--\infty \\
-- \\
-- \\
--\end{array}$ & $\begin{array}{l}167.93 \\
167.93 \\
167.81 \\
167.64 \\
167.66\end{array}$ & $\begin{array}{l}167.99 \\
168.04 \\
168.03 \\
167.66 \\
167.71\end{array}$ & $\begin{array}{l}167.95 \\
167.91 \\
167.70 \\
167.75 \\
167.90\end{array}$ & 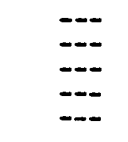 & 167.90 & 167.83 & 167.71 & 166.85 & $\begin{array}{l}--\infty \\
--\infty \\
--\infty \\
--\infty \\
--\infty\end{array}$ & $\begin{array}{r}166.61 \\
\ldots \\
\ldots \\
\ldots \\
-\end{array}$ & 163.58 \\
\hline $\begin{array}{r}6 \\
7 \\
8 \\
9 \\
10\end{array}$ & $\begin{array}{l}167.93 \\
168.00 \\
167.96 \\
167.87 \\
167.89\end{array}$ & $\begin{array}{l}167.93 \\
167.81 \\
167.67 \\
167.65 \\
167.87\end{array}$ & $\begin{array}{l}167.97 \\
168.17 \\
168.03 \\
167.81 \\
167.95\end{array}$ & $\begin{array}{l}167.93 \\
167.71 \\
167.46 \\
167.74 \\
167.82\end{array}$ & $\begin{array}{r}167.92 \\
- \\
- \\
- \\
-\end{array}$ & $\begin{array}{l}--- \\
--- \\
--- \\
--\infty \\
--\infty\end{array}$ & $\begin{array}{l}--\infty \\
--\infty \\
--\infty \\
--\infty \\
--\infty\end{array}$ & $\begin{array}{l}--- \\
--- \\
--- \\
--\infty\end{array}$ & $\begin{array}{l}-- \\
-- \\
--\infty \\
-\infty \\
--\infty\end{array}$ & $\begin{array}{l}-- \\
-- \\
--\infty \\
--\infty \\
--\infty\end{array}$ & $\begin{array}{l}--\infty \\
--\infty \\
--\infty \\
-- \\
--\end{array}$ & $\begin{array}{l}-- \\
-- \\
-- \\
-- \\
--\end{array}$ \\
\hline $\begin{array}{l}11 \\
12 \\
13 \\
14 \\
15\end{array}$ & $\begin{array}{l}167.75 \\
167.96 \\
167.94 \\
167.88 \\
167.80\end{array}$ & $\begin{array}{l}167.86 \\
167.99 \\
167.79 \\
167.88 \\
167.90\end{array}$ & $\begin{array}{l}168.04 \\
167.95 \\
167.91 \\
167.97 \\
168.00\end{array}$ & $\begin{array}{l}-- \\
--\infty \\
-\infty \\
-\infty \\
-\infty\end{array}$ & $\begin{array}{l}-- \\
--\infty \\
-\infty \\
--\infty \\
--\infty\end{array}$ & $\begin{array}{l}-- \\
-- \\
-- \\
-- \\
--\end{array}$ & $\begin{array}{l}--- \\
--- \\
--\infty \\
--\infty\end{array}$ & $\begin{array}{l}--\infty \\
--\infty \\
-\infty \\
-\infty \\
--\infty\end{array}$ & $\begin{array}{l}--\infty \\
--\infty \\
--\infty \\
-\infty\end{array}$ & $\begin{array}{l}--- \\
--\infty \\
--\infty \\
--\infty \\
--\infty\end{array}$ & $\begin{array}{l}-\infty \\
-\infty \\
=-- \\
-\infty\end{array}$ & $\begin{array}{l}-\dot{-} \\
--- \\
--- \\
---\end{array}$ \\
\hline $\begin{array}{l}16 \\
17 \\
18 \\
19 \\
20\end{array}$ & $\begin{array}{l}167.97 \\
168.09 \\
168.16 \\
168.02 \\
167.80\end{array}$ & $\begin{array}{l}167.95 \\
167.87 \\
168.09 \\
167.91 \\
167.96\end{array}$ & $\begin{array}{l}168.03 \\
167.98 \\
168.05 \\
168.02 \\
168.03\end{array}$ & $\begin{array}{l}--0 \\
--- \\
--- \\
--\infty \\
--\end{array}$ & $\begin{array}{l}--- \\
--- \\
--- \\
--- \\
--\end{array}$ & $\begin{array}{l}-- \\
-- \\
-- \\
-- \\
--\end{array}$ & $\begin{array}{l}--- \\
--- \\
--- \\
--- \\
---\end{array}$ & $\begin{array}{l}--\infty \\
--\infty \\
-\infty \\
--\infty \\
--\end{array}$ & $\begin{array}{l}--\infty \\
-- \\
-\infty \\
--\infty \\
--\infty\end{array}$ & $\begin{array}{l}-- \\
-- \\
--- \\
--\infty \\
---\end{array}$ & $\begin{array}{l}-- \\
-- \\
-- \\
--\end{array}$ & $\begin{array}{l}-- \\
-- \\
-- \\
-- \\
--\end{array}$ \\
\hline $\begin{array}{l}21 \\
22 \\
23 \\
24 \\
25\end{array}$ & $\begin{array}{l}167.88 \\
167.97 \\
167.97 \\
167.99 \\
167.99\end{array}$ & $\begin{array}{l}168.05 \\
168.07 \\
168.05 \\
167.85 \\
167.86\end{array}$ & $\begin{array}{l}168.14 \\
168.13 \\
167.98 \\
167.81 \\
167.67\end{array}$ & $\begin{array}{l}--\infty \\
-\infty \\
-\infty \\
-\infty \\
-\infty\end{array}$ & $\begin{array}{l}-- \\
-\infty \\
-\infty \\
-\infty \\
-\infty\end{array}$ & $\begin{array}{l}-- \\
-- \\
-- \\
--\end{array}$ & $\begin{array}{l}--- \\
--- \\
-- \\
-- \\
--\infty\end{array}$ & $\begin{array}{l}--- \\
--- \\
--- \\
--- \\
---\end{array}$ & $\begin{array}{l}-- \\
-- \\
-- \\
-- \\
---\end{array}$ & $\begin{array}{l}-- \\
-- \\
-- \\
-- \\
---\end{array}$ & $\begin{array}{l}-- \\
-- \\
-- \\
--\end{array}$ & $\begin{array}{l}-- \\
-- \\
--- \\
--- \\
--\end{array}$ \\
\hline $\begin{array}{l}26 \\
27 \\
28 \\
29 \\
30 \\
31\end{array}$ & $\begin{array}{l}167.98 \\
167.96 \\
167.95 \\
167.90 \\
167.80 \\
167.82\end{array}$ & $\begin{array}{l}167.97 \\
167.74 \\
168.17 \\
168.08 \\
168.04\end{array}$ & $\begin{array}{l}167.87 \\
167.75 \\
167.80 \\
167.91 \\
167.90 \\
167.76\end{array}$ & 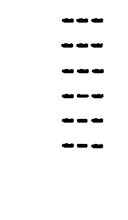 & $\begin{array}{c}-\infty \\
-\infty\end{array}$ & BS4-A & $\begin{array}{l}-- \\
--\infty \\
--\infty \\
--\infty\end{array}$ & 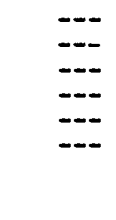 & $\begin{array}{l}-- \\
-- \\
--- \\
-- \\
--\end{array}$ & $\begin{array}{l}-- \\
-- \\
-- \\
-- \\
-- \\
-- \\
.\end{array}$ & $\begin{array}{l}-- \\
-- \\
-- \\
-- \\
-\infty \\
--\end{array}$ & $\begin{array}{l}-- \\
--- \\
-- \\
-- \\
--\end{array}$ \\
\hline $\begin{array}{l}1 \\
2 \\
3 \\
4 \\
5\end{array}$ & $\begin{array}{l}219.54 \\
219.76 \\
220.11 \\
219.98 \\
219.49\end{array}$ & $\begin{array}{l}220.35 \\
220.38 \\
220.23 \\
219.97 \\
219.91\end{array}$ & $\begin{array}{l}220.68 \\
220.72 \\
220.87 \\
220.30 \\
220.38\end{array}$ & $\begin{array}{l}221.24 \\
221.14 \\
221.04 \\
221.22 \\
221.33\end{array}$ & $\begin{array}{l}221 \cdot 44 \\
221.58 \\
221.58 \\
221.53 \\
221.43\end{array}$ & $\begin{array}{l}221.40 \\
221.21 \\
221.39 \\
221.28 \\
221.45\end{array}$ & $\begin{array}{l}219.59 \\
219.78 \\
219.81 \\
219.52 \\
219.64\end{array}$ & $\begin{array}{l}220.14 \\
220.19 \\
220.21 \\
220.03 \\
220.00\end{array}$ & $\begin{array}{l}219.47 \\
219.16 \\
219.27 \\
219.50 \\
219.19\end{array}$ & $\begin{array}{l}216.94 \\
216.75 \\
216.49 \\
216.53 \\
216.61\end{array}$ & $\begin{array}{l}215.97 \\
215.88 \\
215.77 \\
215.78 \\
215.97\end{array}$ & $\begin{array}{l}--\infty \\
--\infty \\
--- \\
--\infty \\
---\end{array}$ \\
\hline $\begin{array}{r}6 \\
7 \\
8 \\
9 \\
10\end{array}$ & $\begin{array}{l}219.71 \\
219.91 \\
219.83 \\
219.72 \\
219.72\end{array}$ & $\begin{array}{l}220.30 \\
220.17 \\
220.03 \\
220.03 \\
220.28\end{array}$ & $\begin{array}{l}220.74 \\
221.15 \\
220.99 \\
220.60 \\
220.79\end{array}$ & $\begin{array}{l}221.37 \\
221.17 \\
220.95 \\
221.10 \\
221.24\end{array}$ & $\begin{array}{l}221 \cdot 51 \\
221 \cdot 50 \\
221 \cdot 26 \\
221.37 \\
221.41\end{array}$ & $\begin{array}{l}221.64 \\
221.63 \\
221.22 \\
221.19 \\
221.18\end{array}$ & $\begin{array}{l}219.78 \\
219.85 \\
219.75 \\
219.67 \\
219.73\end{array}$ & $\begin{array}{l}219.99 \\
219.84 \\
219.89 \\
219.77 \\
219.94\end{array}$ & $\begin{array}{l}219.20 \\
219.22 \\
219.06 \\
219.14 \\
219.24\end{array}$ & $\begin{array}{l}216.58 \\
216.37 \\
216.24 \\
216.34 \\
216.31\end{array}$ & $\begin{array}{l}216.06 \\
215.95 \\
215.85 \\
215.79 \\
215.75\end{array}$ & $\begin{array}{l}--- \\
--\infty \\
--\infty \\
--\infty \\
--\infty\end{array}$ \\
\hline $\begin{array}{l}11 \\
12 \\
13 \\
14 \\
15\end{array}$ & $\begin{array}{l}219.60 \\
219.92 \\
219.95 \\
219.84 \\
219.76\end{array}$ & $\begin{array}{l}220.30 \\
220.49 \\
220.19 \\
220.29 \\
220.32\end{array}$ & $\begin{array}{l}221.02 \\
220.90 \\
220.79 \\
220.92 \\
221.00\end{array}$ & $\begin{array}{l}221.11 \\
221 \cdot 52 \\
221 \cdot 55 \\
221 \cdot 21 \\
221.36\end{array}$ & $\begin{array}{l}221.46 \\
221 \cdot 29 \\
221 \cdot 47 \\
221 \cdot 74 \\
221.37\end{array}$ & $\begin{array}{l}221.02 \\
220.86 \\
220.91 \\
220.57 \\
220.43\end{array}$ & $\begin{array}{l}219.91 \\
219.88 \\
219.79 \\
219.67 \\
219.71\end{array}$ & $\begin{array}{l}220.17 \\
220.11 \\
220.20 \\
220.12 \\
220.07\end{array}$ & $\begin{array}{l}219.08 \\
218.77 \\
218.76 \\
218.87 \\
218.81\end{array}$ & $\begin{array}{l}216.22 \\
216.30 \\
216.29 \\
216.00 \\
215.90\end{array}$ & $\begin{array}{r}215.76 \\
215.79 \\
\\
-\end{array}$ & $\begin{array}{l}-\infty \\
-\infty \\
-\infty \\
-\infty\end{array}$ \\
\hline $\begin{array}{l}16 \\
17 \\
18 \\
19 \\
20\end{array}$ & $\begin{array}{l}220.03 \\
220.30 \\
220.44 \\
220.21 \\
219.85\end{array}$ & $\begin{array}{l}220.43 \\
220.31 \\
220.65 \\
220.40 \\
220.42\end{array}$ & $\begin{array}{l}221.07 \\
220.98 \\
221.12 \\
221.13 \\
221.16\end{array}$ & $\begin{array}{l}221.39 \\
221.33 \\
221.63 \\
221.60 \\
221.22\end{array}$ & $\begin{array}{l}221 \cdot 32 \\
221 \cdot 79 \\
221 \cdot 51 \\
221 \cdot 75 \\
221.73\end{array}$ & $\begin{array}{l}220.52 \\
220.45 \\
220.60 \\
220.76 \\
220.58\end{array}$ & $\begin{array}{l}219.68 \\
220.00 \\
220.10 \\
219.85 \\
219.79\end{array}$ & $\begin{array}{l}219.91 \\
220.07 \\
220.24 \\
219.95 \\
220.12\end{array}$ & $\begin{array}{l}218.62 \\
218.49 \\
218.56 \\
218.35 \\
218.18\end{array}$ & $\begin{array}{l}215.97 \\
216.03 \\
216.08 \\
216.01 \\
215.96\end{array}$ & $\begin{array}{l}--\infty \\
--- \\
--- \\
--- \\
---\end{array}$ & $\begin{array}{l}--\infty \\
--\infty \\
--\infty \\
--\infty \\
--\infty\end{array}$ \\
\hline $\begin{array}{l}21 \\
22 \\
23 \\
24 \\
25\end{array}$ & $\begin{array}{l}219.91 \\
220.11 \\
220.14 \\
220.19 \\
220.21\end{array}$ & $\begin{array}{l}220.62 \\
220.62 \\
220.61 \\
220.35 \\
220.35\end{array}$ & $\begin{array}{l}221.37 \\
221.49 \\
221.30 \\
220.90 \\
220.67\end{array}$ & $\begin{array}{l}221.16 \\
221.23 \\
220.99 \\
221.20 \\
221.22\end{array}$ & $\begin{array}{l}221 \cdot 39 \\
221 \cdot 13 \\
221 \cdot 16 \\
221 \cdot 58 \\
221.88\end{array}$ & $\begin{array}{l}220.20 \\
220.13 \\
220.54 \\
220.39 \\
220.16\end{array}$ & $\begin{array}{l}219.92 \\
219.81 \\
219.65 \\
219.65 \\
219.80\end{array}$ & $\begin{array}{l}220.29 \\
220.28 \\
220.10 \\
220.04 \\
219.89\end{array}$ & $\begin{array}{l}218.14 \\
217.88 \\
217.87 \\
217.82 \\
217.71\end{array}$ & $\begin{array}{l}215.99 \\
215.94 \\
215.94 \\
215.97 \\
216.00\end{array}$ & $\begin{array}{l}--\infty \\
-- \\
-- \\
-\sim \\
--\end{array}$ & $\begin{array}{l}-- \\
-- \\
-- \\
-- \\
--\end{array}$ \\
\hline $\begin{array}{l}26 \\
27 \\
28 \\
29 \\
30 \\
31\end{array}$ & $\begin{array}{l}220.23 \\
220.21 \\
220.20 \\
220.13 \\
220.09 \\
220.15\end{array}$ & $\begin{array}{l}220.54 \\
220.27 \\
220.86 \\
220.83 \\
220.74\end{array}$ & $\begin{array}{l}220.96 \\
220.85 \\
220.97 \\
221.09 \\
221.06 \\
220.93\end{array}$ & $\begin{array}{l}221.30 \\
221.29 \\
221.59 \\
221.30 \\
221.35 \\
221.49\end{array}$ & $\begin{array}{l}221.52 \\
221.65 \\
221.70\end{array}$ & $\begin{array}{l}220.25 \\
220.13 \\
219.98 \\
219.88 \\
219.80 \\
219.71\end{array}$ & $\begin{array}{l}219.73 \\
219.64 \\
219.57 \\
219.78 \\
220.03\end{array}$ & $\begin{array}{l}219.87 \\
219.82 \\
219.76 \\
219.77 \\
219.80 \\
219.70\end{array}$ & $\begin{array}{l}217.52 \\
217.36 \\
217.12 \\
217.04 \\
216.99\end{array}$ & $\begin{array}{l}216.01 \\
215.93 \\
215.84 \\
215.77 \\
215.91 \\
216.00\end{array}$ & 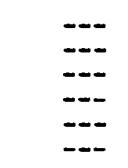 & $\begin{array}{l}-\infty \\
-\infty \\
--\infty \\
-\infty\end{array}$ \\
\hline
\end{tabular}


Table 26. Daily mean discharge and specific conductance at Big Spring, Clayton County, Iowa, water year 1990

[--, data not available to calculate mean values]

\begin{tabular}{|c|c|c|c|c|c|c|c|c|c|c|c|c|}
\hline Day & Oct. & Nov. & Dec. & Jan. & Feb. & Mar. & Apr. & May & June & July & Aug. & Sept. \\
\hline \multicolumn{13}{|c|}{ Daily wean discharge, in cubic feet per second } \\
\hline $\begin{array}{l}1 \\
2 \\
3 \\
4 \\
5\end{array}$ & $\begin{array}{l}12 \\
12 \\
12 \\
12 \\
11\end{array}$ & $\begin{array}{l}12 \\
12 \\
12 \\
12 \\
12\end{array}$ & $\begin{array}{l}10 \\
10 \\
10 \\
10 \\
10\end{array}$ & $\begin{array}{l}9.5 \\
9.6 \\
9.6 \\
9.7 \\
9.7\end{array}$ & $\begin{array}{l}10 \\
10 \\
10 \\
10 \\
24\end{array}$ & $\begin{array}{l}13 \\
26 \\
18 \\
15 \\
28\end{array}$ & $\begin{array}{l}16 \\
16 \\
16 \\
16 \\
16\end{array}$ & $\begin{array}{l}16 \\
15 \\
15 \\
16 \\
15\end{array}$ & $\begin{array}{l}21 \\
27 \\
35 \\
27 \\
26\end{array}$ & $\begin{array}{l}36 \\
35 \\
34 \\
30 \\
29\end{array}$ & $\begin{array}{l}21 \\
21 \\
20 \\
24 \\
22\end{array}$ & $\begin{array}{l}48 \\
42 \\
41 \\
39 \\
35\end{array}$ \\
\hline $\begin{array}{r}6 \\
7 \\
8 \\
9 \\
10\end{array}$ & $\begin{array}{l}11 \\
11 \\
11 \\
10 \\
10\end{array}$ & $\begin{array}{l}11 \\
11 \\
11 \\
11 \\
11\end{array}$ & $\begin{array}{l}10 \\
10 \\
10 \\
10 \\
10\end{array}$ & $\begin{array}{l}9.7 \\
9.7 \\
9.7 \\
24 \\
17\end{array}$ & $\begin{array}{l}36 \\
31 \\
65 \\
38 \\
19\end{array}$ & $\begin{array}{r}33 \\
11 \\
132 \\
87 \\
29\end{array}$ & $\begin{array}{l}16 \\
16 \\
16 \\
16 \\
16\end{array}$ & $\begin{array}{l}15 \\
15 \\
15 \\
16 \\
17\end{array}$ & $\begin{array}{l}25 \\
23 \\
22 \\
21 \\
20\end{array}$ & $\begin{array}{l}29 \\
28 \\
27 \\
26 \\
25\end{array}$ & $\begin{array}{l}20 \\
19 \\
19 \\
19 \\
19\end{array}$ & $\begin{array}{l}31 \\
30 \\
30 \\
28 \\
28\end{array}$ \\
\hline $\begin{array}{l}11 \\
12 \\
13 \\
14 \\
15\end{array}$ & $\begin{array}{l}11 \\
10 \\
10 \\
10 \\
11\end{array}$ & $\begin{array}{l}11 \\
11 \\
11 \\
11 \\
11\end{array}$ & $\begin{array}{l}10 \\
10 \\
9.9 \\
9.8 \\
9.7\end{array}$ & $\begin{array}{l}11 \\
10 \\
10 \\
10 \\
10\end{array}$ & $\begin{array}{l}14 \\
16 \\
21 \\
13 \\
13\end{array}$ & $\begin{array}{l}74 \\
37 \\
31 \\
65 \\
42\end{array}$ & $\begin{array}{l}16 \\
16 \\
15 \\
16 \\
16\end{array}$ & $\begin{array}{l}16 \\
16 \\
16 \\
16 \\
15\end{array}$ & $\begin{array}{l}20 \\
20 \\
23 \\
24 \\
22\end{array}$ & $\begin{array}{l}25 \\
24 \\
23 \\
22 \\
22\end{array}$ & $\begin{array}{l}18 \\
18 \\
18 \\
18 \\
17\end{array}$ & $\begin{array}{l}28 \\
29 \\
28 \\
29 \\
28\end{array}$ \\
\hline $\begin{array}{l}16 \\
17 \\
18 \\
19 \\
20\end{array}$ & $\begin{array}{l}12 \\
12 \\
11 \\
12 \\
11\end{array}$ & $\begin{array}{l}11 \\
11 \\
10 \\
11 \\
11\end{array}$ & $\begin{array}{l}9.7 \\
9.7 \\
9.6 \\
9.6 \\
9.6\end{array}$ & $\begin{array}{l}11 \\
49 \\
14 \\
11 \\
11\end{array}$ & $\begin{array}{l}12 \\
12 \\
12 \\
11 \\
11\end{array}$ & $\begin{array}{l}31 \\
26 \\
23 \\
21 \\
20\end{array}$ & $\begin{array}{l}15 \\
15 \\
15 \\
15 \\
16\end{array}$ & $\begin{array}{r}16 \\
16 \\
15 \\
24 \\
41\end{array}$ & $\begin{array}{l}28 \\
34 \\
35 \\
33 \\
31\end{array}$ & $\begin{array}{l}21 \\
20 \\
22 \\
30 \\
33\end{array}$ & $\begin{array}{l}17 \\
18 \\
19 \\
25 \\
25\end{array}$ & $\begin{array}{l}27 \\
25 \\
26 \\
26 \\
25\end{array}$ \\
\hline $\begin{array}{l}21 \\
22 \\
23 \\
24 \\
25\end{array}$ & $\begin{array}{l}11 \\
11 \\
11 \\
11 \\
11\end{array}$ & $\begin{array}{l}11 \\
11 \\
11 \\
11 \\
11\end{array}$ & $\begin{array}{l}9.5 \\
9.5 \\
9.5 \\
9.5 \\
9.5\end{array}$ & $\begin{array}{l}10 \\
10 \\
10 \\
10 \\
10\end{array}$ & $\begin{array}{l}11 \\
11 \\
11 \\
11 \\
11\end{array}$ & $\begin{array}{l}29 \\
20 \\
19 \\
18 \\
18\end{array}$ & $\begin{array}{l}16 \\
17 \\
17 \\
17 \\
17\end{array}$ & $\begin{array}{l}37 \\
33 \\
29 \\
28 \\
27\end{array}$ & $\begin{array}{l}30 \\
62 \\
68 \\
58 \\
51\end{array}$ & $\begin{array}{l}26 \\
25 \\
23 \\
21 \\
21\end{array}$ & $\begin{array}{r}24 \\
22 \\
21 \\
23 \\
187\end{array}$ & $\begin{array}{l}26 \\
25 \\
24 \\
24 \\
25\end{array}$ \\
\hline \multirow[t]{2}{*}{$\begin{array}{l}26 \\
27 \\
28 \\
29 \\
30 \\
31\end{array}$} & $\begin{array}{l}11 \\
11 \\
11 \\
11 \\
11 \\
12\end{array}$ & $\begin{array}{l}11 \\
11 \\
11 \\
11 \\
10\end{array}$ & $\begin{array}{l}9.4 \\
9.5 \\
9.5 \\
9.5 \\
9.5 \\
9.5\end{array}$ & $\begin{array}{l}10 \\
10 \\
10 \\
10 \\
10 \\
10\end{array}$ & $\begin{array}{l}10 \\
10 \\
10\end{array}$ & $\begin{array}{l}18 \\
18 \\
17 \\
17 \\
17 \\
17\end{array}$ & $\begin{array}{l}17 \\
17 \\
17 \\
17 \\
16\end{array}$ & $\begin{array}{l}26 \\
24 \\
24 \\
24 \\
22 \\
21\end{array}$ & $\begin{array}{l}60 \\
49 \\
44 \\
41 \\
38\end{array}$ & $\begin{array}{l}20 \\
27 \\
27 \\
25 \\
24 \\
22\end{array}$ & $\begin{array}{r}117 \\
96 \\
71 \\
62 \\
55 \\
51\end{array}$ & $\begin{array}{l}23 \\
24 \\
24 \\
23 \\
23\end{array}$ \\
\hline & \multicolumn{12}{|c|}{ Daily mean specific conductance, in microsiemens per centimeter at 25 degrees Celsius } \\
\hline $\begin{array}{l}1 \\
2 \\
3 \\
4 \\
5\end{array}$ & \begin{tabular}{l}
750 \\
750 \\
--- \\
\hdashline--
\end{tabular} & $\begin{array}{l}745 \\
744 \\
744 \\
742 \\
744\end{array}$ & $\begin{array}{l}764 \\
762 \\
762 \\
760 \\
760\end{array}$ & $\begin{array}{l}750 \\
751 \\
752 \\
754 \\
753\end{array}$ & $\begin{array}{l}742 \\
740 \\
744 \\
763 \\
789\end{array}$ & $\begin{array}{l}682 \\
700 \\
736 \\
723 \\
681\end{array}$ & 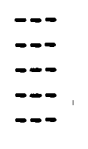 & $\begin{array}{l}722 \\
724 \\
725 \\
726 \\
726\end{array}$ & $\begin{array}{l}743 \\
743 \\
741 \\
742 \\
---\end{array}$ & $\begin{array}{l}736 \\
747 \\
752 \\
753 \\
753\end{array}$ & $\begin{array}{l}755 \\
756 \\
756 \\
754 \\
758\end{array}$ & $\begin{array}{l}752 \\
759 \\
760 \\
766 \\
766\end{array}$ \\
\hline $\begin{array}{r}6 \\
7 \\
8 \\
9 \\
10\end{array}$ & $\begin{array}{l}--- \\
--- \\
--- \\
--\end{array}$ & $\begin{array}{l}742 \\
740 \\
741 \\
748 \\
752\end{array}$ & $\begin{array}{l}759 \\
758 \\
757 \\
758 \\
756\end{array}$ & $\begin{array}{l}751 \\
751 \\
761 \\
769 \\
778\end{array}$ & $\begin{array}{l}796 \\
770 \\
676 \\
539 \\
481\end{array}$ & $\begin{array}{l}654 \\
647 \\
635 \\
378 \\
392\end{array}$ & 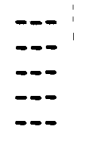 & $\begin{array}{l}726 \\
728 \\
728 \\
729 \\
729\end{array}$ & $\begin{array}{l}733 \\
736 \\
735 \\
729 \\
722\end{array}$ & \begin{tabular}{l}
754 \\
\hdashline 754 \\
755 \\
755
\end{tabular} & $\begin{array}{l}760 \\
755 \\
754 \\
752 \\
746\end{array}$ & $\begin{array}{l}763 \\
764 \\
768 \\
766 \\
766\end{array}$ \\
\hline $\begin{array}{l}11 \\
12 \\
13 \\
14 \\
15\end{array}$ & $\begin{array}{l}752 \\
750 \\
749 \\
755 \\
764\end{array}$ & $\begin{array}{l}752 \\
750 \\
748 \\
747 \\
747\end{array}$ & $\begin{array}{l}754 \\
752 \\
751 \\
752 \\
753\end{array}$ & $\begin{array}{l}787 \\
786 \\
781 \\
766 \\
742\end{array}$ & $\begin{array}{l}500 \\
516 \\
539 \\
552 \\
545\end{array}$ & $\begin{array}{l}452 \\
438 \\
469 \\
540 \\
537\end{array}$ & $\begin{array}{l}727 \\
729 \\
725 \\
724 \\
723\end{array}$ & $\begin{array}{l}730 \\
728 \\
724 \\
727 \\
729\end{array}$ & $\begin{array}{l}723 \\
730 \\
736 \\
742 \\
746\end{array}$ & $\begin{array}{l}756 \\
757 \\
756 \\
753 \\
753\end{array}$ & $\begin{array}{l}750 \\
755 \\
751 \\
755 \\
757\end{array}$ & $\begin{array}{l}766 \\
765 \\
764 \\
773 \\
762\end{array}$ \\
\hline $\begin{array}{l}16 \\
17 \\
18 \\
19 \\
20\end{array}$ & $\begin{array}{l}774 \\
778 \\
775 \\
771 \\
759\end{array}$ & $\begin{array}{l}748 \\
751 \\
754 \\
753 \\
751\end{array}$ & $\begin{array}{l}754 \\
754 \\
755 \\
758 \\
759\end{array}$ & $\begin{array}{l}734 \\
733 \\
728 \\
721 \\
694\end{array}$ & $\begin{array}{l}549 \\
560 \\
571 \\
583 \\
592\end{array}$ & $\begin{array}{l}572 \\
601 \\
619 \\
632 \\
646\end{array}$ & $\begin{array}{l}722 \\
720 \\
720 \\
722 \\
722\end{array}$ & $\begin{array}{l}729 \\
731 \\
734 \\
734 \\
---\end{array}$ & $\begin{array}{l}741 \\
743 \\
733 \\
727 \\
729\end{array}$ & $\begin{array}{l}753 \\
751 \\
754 \\
747 \\
739\end{array}$ & $\begin{array}{l}758 \\
756 \\
758 \\
756 \\
757\end{array}$ & $\begin{array}{l}761 \\
761 \\
759 \\
760 \\
759\end{array}$ \\
\hline $\begin{array}{l}21 \\
22 \\
23 \\
24 \\
25\end{array}$ & $\begin{array}{l}752 \\
747 \\
743 \\
741 \\
742\end{array}$ & $\begin{array}{l}750 \\
751 \\
755 \\
756 \\
759\end{array}$ & $\begin{array}{l}758 \\
758 \\
759 \\
759 \\
759\end{array}$ & $\begin{array}{l}651 \\
655 \\
682 \\
693 \\
687\end{array}$ & $\begin{array}{l}604 \\
618 \\
635 \\
643 \\
662\end{array}$ & $\begin{array}{l}664 \\
673 \\
679 \\
689 \\
698\end{array}$ & $\begin{array}{l}721 \\
720 \\
721 \\
721 \\
720\end{array}$ & \begin{tabular}{l}
--- \\
\hdashline-- \\
743 \\
741
\end{tabular} & $\begin{array}{l}739 \\
738 \\
717 \\
725 \\
726\end{array}$ & $\begin{array}{l}-7- \\
731 \\
737 \\
745 \\
740\end{array}$ & $\begin{array}{l}753 \\
748 \\
750 \\
753 \\
659\end{array}$ & $\begin{array}{l}748 \\
747 \\
750 \\
748 \\
741\end{array}$ \\
\hline $\begin{array}{l}26 \\
27 \\
28 \\
29 \\
30 \\
31\end{array}$ & $\begin{array}{l}742 \\
746 \\
748 \\
748 \\
746 \\
746\end{array}$ & $\begin{array}{l}761 \\
761 \\
761 \\
761 \\
763\end{array}$ & $\begin{array}{l}759 \\
760 \\
759 \\
757 \\
757 \\
-. .\end{array}$ & $\begin{array}{l}688 \\
692 \\
702 \\
717 \\
733 \\
743\end{array}$ & $\begin{array}{l}679 \\
678 \\
676\end{array}$ & $\begin{array}{l}707 \\
712 \\
717 \\
--- \\
--\end{array}$ & $\begin{array}{l}721 \\
722 \\
723 \\
723 \\
722\end{array}$ & $\begin{array}{l}750 \\
749 \\
740 \\
742 \\
743 \\
743\end{array}$ & $\begin{array}{l}733 \\
747 \\
722 \\
720 \\
711\end{array}$ & $\begin{array}{l}735 \\
741 \\
751 \\
755 \\
755 \\
756\end{array}$ & $\begin{array}{l}617 \\
660 \\
670 \\
700 \\
723 \\
741\end{array}$ & $\begin{array}{l}745 \\
747 \\
746 \\
748 \\
---\end{array}$ \\
\hline
\end{tabular}


Table 27. Daily median $p H$ and daily mean water temperature at Big Spring, Clayton County, Iowa, water year 1990

[--, data not available to calculate median values]

\begin{tabular}{|c|c|c|c|c|c|c|c|c|c|c|c|c|}
\hline Day & Oct. & Nov. & Dec. & Jan. & Feb. & Mar. & Apr. & May & June & July & Aug. & Sept. \\
\hline \multicolumn{13}{|c|}{ Daily median $\mathrm{pH}$, in standard units } \\
\hline $\begin{array}{l}1 \\
2 \\
3 \\
4 \\
5\end{array}$ & $\begin{array}{l}6.7 \\
6.7 \\
6.8 \\
6.8 \\
6.8\end{array}$ & $\begin{array}{l}6.8 \\
6.8 \\
6.8 \\
6.8 \\
6.8\end{array}$ & $\begin{array}{l}7.0 \\
7.0 \\
7.0 \\
7.0 \\
7.0\end{array}$ & $\begin{array}{l}7.1 \\
7.1 \\
7.1 \\
7.0 \\
7.0\end{array}$ & $\begin{array}{l}6.8 \\
6.8 \\
6.8 \\
6.8 \\
6.8\end{array}$ & $\begin{array}{l}6.8 \\
6.9 \\
6.9 \\
6.8 \\
6.8\end{array}$ & \begin{tabular}{l}
--- \\
\hdashline-- \\
\hdashline-- \\
---
\end{tabular} & $\begin{array}{l}6.9 \\
6.9 \\
6.9 \\
6.9 \\
6.9\end{array}$ & $\begin{array}{l}6.8 \\
6.8 \\
6.8- \\
6.8 \\
-.-\end{array}$ & $\begin{array}{l}6.8 \\
6.8 \\
6.8 \\
6.8 \\
6.8\end{array}$ & $\begin{array}{l}6.7 \\
6.7 \\
6.7 \\
6.7 \\
6.7\end{array}$ & $\begin{array}{l}6.6 \\
6.7 \\
6.7 \\
6.7 \\
6.8\end{array}$ \\
\hline $\begin{array}{r}6 \\
7 \\
8 \\
9 \\
10\end{array}$ & $\begin{array}{l}6.8 \\
6.8 \\
6.8 \\
6.8 \\
6.8\end{array}$ & $\begin{array}{l}6.8 \\
6.8 \\
6.8 \\
6.8 \\
6.7\end{array}$ & $\begin{array}{l}7.0 \\
7.0 \\
7.0 \\
7.0 \\
7.0\end{array}$ & $\begin{array}{l}7.0 \\
7.0 \\
7.0 \\
7.0 \\
6.9\end{array}$ & $\begin{array}{l}6.8 \\
6.8 \\
6.7 \\
6.6 \\
6.5\end{array}$ & $\begin{array}{l}6.9 \\
6.9 \\
6.9 \\
6.6 \\
6.6\end{array}$ & $\begin{array}{l}--- \\
--- \\
--- \\
---\end{array}$ & $\begin{array}{l}6.9 \\
6.9 \\
6.9 \\
6.9 \\
6.9\end{array}$ & $\begin{array}{l}6.8 \\
6.8 \\
6.8 \\
6.8 \\
6.7\end{array}$ & $\begin{array}{l}6.8 \\
-1.8 \\
6.8 \\
6.8\end{array}$ & $\begin{array}{l}6.7 \\
6.7 \\
6.7 \\
6.7 \\
6.7\end{array}$ & $\begin{array}{l}6.8 \\
7.0 \\
7.0 \\
7.0 \\
7.0\end{array}$ \\
\hline $\begin{array}{l}11 \\
12 \\
13 \\
14 \\
15\end{array}$ & $\begin{array}{l}6.8 \\
6.8 \\
6.8 \\
6.8 \\
6.8\end{array}$ & $\begin{array}{l}6.7 \\
6.7 \\
6.7 \\
6.8 \\
6.8\end{array}$ & $\begin{array}{l}7.0 \\
7.0 \\
7.1 \\
7.1 \\
7.1\end{array}$ & $\begin{array}{l}6.9 \\
6.9 \\
6.9 \\
6.9 \\
6.8\end{array}$ & $\begin{array}{l}6.6 \\
6.6 \\
6.6 \\
6.6 \\
6.6\end{array}$ & $\begin{array}{l}6.7 \\
6.5 \\
6.6 \\
6.7 \\
6.7\end{array}$ & $\begin{array}{l}6.9 \\
6.9 \\
6.9 \\
6.9 \\
6.9\end{array}$ & $\begin{array}{l}6.9 \\
6.9 \\
6.9 \\
6.9 \\
6.9\end{array}$ & $\begin{array}{l}6.8 \\
6.7- \\
6.8 \\
6.8 \\
6.8\end{array}$ & $\begin{array}{l}6.8 \\
6.7 \\
6.7 \\
6.8 \\
6.7\end{array}$ & $\begin{array}{l}6.7 \\
6.7 \\
6.7 \\
6.7 \\
6.7\end{array}$ & $\begin{array}{l}7.0 \\
7.0 \\
7.0 \\
7.0 \\
7.0\end{array}$ \\
\hline $\begin{array}{l}16 \\
17 \\
18 \\
19 \\
20\end{array}$ & $\begin{array}{l}6.8 \\
6.8 \\
6.8 \\
6.8 \\
6.8\end{array}$ & $\begin{array}{l}6.8 \\
6.8 \\
6.9 \\
6.9 \\
7.0\end{array}$ & $\begin{array}{l}7.1 \\
7.1 \\
7.1 \\
7.1 \\
7.2\end{array}$ & $\begin{array}{l}6.8 \\
6.8 \\
6.8 \\
6.7 \\
6.7\end{array}$ & $\begin{array}{l}6.6 \\
6.7 \\
6.7 \\
6.7 \\
6.7\end{array}$ & $\begin{array}{l}6.7 \\
6.7 \\
6.8 \\
6.8 \\
6.8\end{array}$ & $\begin{array}{l}6.9 \\
6.9 \\
6.8 \\
6.8 \\
6.9\end{array}$ & $\begin{array}{l}6.8 \\
6.8 \\
6.8 \\
6.8 \\
6.8\end{array}$ & $\begin{array}{l}6.8 \\
6.8 \\
6.8 \\
6.8 \\
6.8\end{array}$ & $\begin{array}{l}6.7 \\
6.7 \\
6.7 \\
6.7 \\
6.6\end{array}$ & $\begin{array}{l}6.7 \\
6.7 \\
6.7 \\
6.7 \\
6.7\end{array}$ & $\begin{array}{l}7.0 \\
7.0 \\
7.2 \\
7.2 \\
7.2\end{array}$ \\
\hline $\begin{array}{l}21 \\
22 \\
23 \\
24 \\
25\end{array}$ & $\begin{array}{l}6.8 \\
6.8 \\
6.8 \\
6.8 \\
6.8\end{array}$ & $\begin{array}{l}7.0 \\
7.0 \\
7.0 \\
6.9 \\
6.8\end{array}$ & $\begin{array}{l}7.2 \\
7.2 \\
7.2 \\
7.2 \\
7.2\end{array}$ & $\begin{array}{l}6.6 \\
6.6 \\
6.7 \\
6.8 \\
6.8\end{array}$ & $\begin{array}{l}6.7 \\
6.8 \\
6.8 \\
6.8 \\
6.8\end{array}$ & $\begin{array}{l}6.8 \\
6.8 \\
6.8 \\
6.8 \\
6.8\end{array}$ & $\begin{array}{c}6.9 \\
6.8 \\
6.8 \\
-\ldots- \\
-.-\end{array}$ & $\begin{array}{l}6.8 \\
6.8 \\
6.8 \\
6.8 \\
6.8\end{array}$ & $\begin{array}{l}6.8 \\
6.8 \\
6.7 \\
6.7 \\
6.7\end{array}$ & $\begin{array}{l}6.7 \\
6.7 \\
6.7 \\
6.7 \\
6.7\end{array}$ & $\begin{array}{l}6.5 \\
6.5 \\
6.7 \\
6.7 \\
6.7\end{array}$ & $\begin{array}{l}6.8 \\
6.8 \\
6.8 \\
6.8 \\
6.8\end{array}$ \\
\hline $\begin{array}{l}26 \\
27 \\
28 \\
29 \\
30 \\
31\end{array}$ & $\begin{array}{l}6.8 \\
6.8 \\
6.8 \\
6.8 \\
6.8 \\
6.8\end{array}$ & $\begin{array}{l}7.0 \\
7.0 \\
7.0 \\
7.0 \\
7.0\end{array}$ & $\begin{array}{l}7.2 \\
7.2 \\
7.1 \\
7.1 \\
7.1 \\
-.-\end{array}$ & $\begin{array}{l}6.7 \\
6.7 \\
6.7 \\
6.7 \\
6.8 \\
6.8\end{array}$ & $\begin{array}{l}6.8 \\
6.8 \\
6.8\end{array}$ & $\begin{array}{c}6.8 \\
6.8 \\
6.8 \\
--- \\
---\end{array}$ & $\begin{array}{l}6.9 \\
7.0 \\
7.0 \\
7.0 \\
7.0\end{array}$ & $\begin{array}{l}6.8 \\
6.8 \\
6.8 \\
6.8 \\
6.8 \\
6.8\end{array}$ & $\begin{array}{l}6.8 \\
6.8 \\
6.8 \\
6.8 \\
6.8\end{array}$ & $\begin{array}{l}6.7 \\
6.7 \\
6.7 \\
6.7 \\
6.7 \\
6.7\end{array}$ & $\begin{array}{l}6.6 \\
6.6 \\
6.7 \\
6.7 \\
6.6 \\
6.6\end{array}$ & $\begin{array}{c}6.8 \\
6.8 \\
-1.8\end{array}$ \\
\hline
\end{tabular}

Daily mean water temperature, in degrees Celsius

\begin{tabular}{|c|c|c|c|c|c|c|c|c|c|c|c|}
\hline $\begin{array}{l}10.5 \\
10.5 \\
10.5 \\
10.5 \\
10.5\end{array}$ & $\begin{array}{l}10.5 \\
10.5 \\
10.5 \\
10.5 \\
10.0\end{array}$ & $\begin{array}{l}10.0 \\
10.0 \\
10.0 \\
10.0 \\
10.0\end{array}$ & $\begin{array}{l}10.0 \\
10.0 \\
10.0 \\
10.0 \\
10.0\end{array}$ & $\begin{array}{l}9.0 \\
9.0 \\
9.5 \\
9.5 \\
9.5\end{array}$ & $\begin{array}{l}9.0 \\
9.0 \\
9.0 \\
9.0 \\
9.0\end{array}$ & $\begin{array}{l}=- \\
--- \\
-- \\
-\cdots\end{array}$ & $\begin{array}{l}9.0 \\
9.0 \\
9.0 \\
9.0 \\
9.0\end{array}$ & $\begin{array}{l}9.5 \\
9.5 \\
9.5 \\
9.5 \\
-.-\end{array}$ & $\begin{array}{l}10.0 \\
10.0 \\
10.0 \\
10.0 \\
10.0\end{array}$ & $\begin{array}{l}10.5 \\
10.5 \\
10.5 \\
10.5 \\
10.5\end{array}$ & $\begin{array}{l}11.0 \\
11.0 \\
11.0 \\
11.0 \\
11.0\end{array}$ \\
\hline $\begin{array}{l}10.5 \\
10.5 \\
10.5 \\
10.5 \\
10.5\end{array}$ & $\begin{array}{l}10.0 \\
10.0 \\
10.0 \\
10.0 \\
10.0\end{array}$ & $\begin{array}{l}10.0 \\
10.0 \\
10.0 \\
10.0 \\
10.0\end{array}$ & $\begin{array}{r}10.0 \\
10.0 \\
10.0 \\
10.0 \\
9.5\end{array}$ & $\begin{array}{l}9.0 \\
9.0 \\
8.5 \\
8.0 \\
7.5\end{array}$ & $\begin{array}{l}9.0 \\
9.0 \\
8.5 \\
5.5 \\
6.0\end{array}$ & $\begin{array}{l}--- \\
-- \\
-- \\
--- \\
-\end{array}$ & $\begin{array}{l}9.0 \\
9.0 \\
9.0 \\
9.0 \\
9.0\end{array}$ & $\begin{array}{l}9.5 \\
9.5 \\
9.5 \\
9.5 \\
9.5\end{array}$ & $\begin{array}{r}10.0 \\
10.0 \\
10.0 \\
10.0\end{array}$ & $\begin{array}{l}10.5 \\
10.0 \\
10.0 \\
10.0 \\
10.0\end{array}$ & $\begin{array}{l}11.0 \\
10.5 \\
10.5 \\
10.5 \\
10.5\end{array}$ \\
\hline $\begin{array}{l}10.5 \\
10.5 \\
10.5 \\
10.5 \\
10.5\end{array}$ & $\begin{array}{l}10.0 \\
10.0 \\
10.0 \\
10.0 \\
10.0\end{array}$ & $\begin{array}{l}10.0 \\
10.0 \\
10.0 \\
10.0 \\
10.0\end{array}$ & $\begin{array}{l}9.5 \\
9.5 \\
9.5 \\
9.5 \\
9.5\end{array}$ & $\begin{array}{l}8.0 \\
8.0 \\
8.0 \\
8.0 \\
8.0\end{array}$ & $\begin{array}{l}6.5 \\
6.5 \\
6.5 \\
7.5 \\
8.0\end{array}$ & $\begin{array}{l}9.0 \\
9.0 \\
9.0 \\
9.0 \\
9.5\end{array}$ & $\begin{array}{l}9.0 \\
9.0 \\
9.0 \\
9.0 \\
9.0\end{array}$ & $\begin{array}{l}9.5 \\
9.5 \\
9.5 \\
9.5 \\
9.5\end{array}$ & $\begin{array}{l}10.0 \\
10.0 \\
10.0 \\
10.0 \\
10.0\end{array}$ & $\begin{array}{l}10.0 \\
10.0 \\
10.0 \\
10.0 \\
10.5\end{array}$ & $\begin{array}{l}10.5 \\
10.5 \\
10.5 \\
10.5 \\
10.5\end{array}$ \\
\hline $\begin{array}{l}10.5 \\
10.5 \\
10.5 \\
10.5 \\
10.5\end{array}$ & $\begin{array}{l}10.0 \\
10.0 \\
10.0 \\
10.0 \\
10.0\end{array}$ & $\begin{array}{l}10.0 \\
10.0 \\
10.0 \\
10.0 \\
10.0\end{array}$ & $\begin{array}{l}9.5 \\
9.5 \\
9.5 \\
9.5 \\
9.5\end{array}$ & $\begin{array}{l}8.0 \\
8.0 \\
8.5 \\
8.5 \\
8.5\end{array}$ & $\begin{array}{l}8.0 \\
8.0 \\
8.5 \\
8.5 \\
8.5\end{array}$ & $\begin{array}{l}9.5 \\
9.5 \\
9.5 \\
9.5 \\
9.5\end{array}$ & $\begin{array}{l}9.0 \\
9.0 \\
9.5 \\
9.5 \\
9.5\end{array}$ & $\begin{array}{r}9.5 \\
9.5 \\
9.5 \\
10.0 \\
10.0\end{array}$ & $\begin{array}{l}10.0 \\
10.0 \\
10.0 \\
10.0 \\
10.5\end{array}$ & $\begin{array}{l}10.0 \\
10.0 \\
10.0 \\
10.5 \\
10.5\end{array}$ & $\begin{array}{l}10.5 \\
10.5 \\
10.5 \\
10.5 \\
10.5\end{array}$ \\
\hline $\begin{array}{l}10.5 \\
10.5 \\
10.5 \\
10.5 \\
10.5\end{array}$ & $\begin{array}{l}10.0 \\
10.0 \\
10.0 \\
10.0 \\
10.0\end{array}$ & $\begin{array}{l}10.0 \\
10.0 \\
10.0 \\
10.0 \\
10.0\end{array}$ & $\begin{array}{l}9.0 \\
9.0 \\
9.0 \\
9.0 \\
9.0\end{array}$ & $\begin{array}{l}8.5 \\
8.5 \\
8.5 \\
8.5 \\
8.5\end{array}$ & $\begin{array}{l}8.5 \\
8.5 \\
8.5 \\
9.0 \\
9.0\end{array}$ & $\begin{array}{l}9.5 \\
9.5 \\
9.5 \\
---\end{array}$ & $\begin{array}{l}9.5 \\
9.5 \\
9.5 \\
9.5 \\
9.5\end{array}$ & $\begin{array}{l}10.0 \\
10.0 \\
10.0 \\
10.0 \\
10.0\end{array}$ & $\begin{array}{r}--. \\
10.0 \\
10.5 \\
10.0 \\
10.0\end{array}$ & $\begin{array}{l}10.5 \\
10.5 \\
10.5 \\
10.5 \\
11.0\end{array}$ & $\begin{array}{l}10.5 \\
10.5 \\
10.5 \\
10.5 \\
10.5\end{array}$ \\
\hline $\begin{array}{l}10.5 \\
10.0 \\
10.0 \\
10.0 \\
10.0 \\
10.0\end{array}$ & $\begin{array}{l}10.0 \\
10.0 \\
10.0 \\
10.0 \\
10.0\end{array}$ & $\begin{array}{r}10.0 \\
10.0 \\
10.0 \\
10.0 \\
10.0 \\
-\end{array}$ & $\begin{array}{l}9.0 \\
9.0 \\
9.0 \\
9.0 \\
9.0 \\
9.0\end{array}$ & $\begin{array}{l}8.5 \\
8.5 \\
9.0\end{array}$ & $\begin{array}{l}9.0 \\
9.0 \\
9.0 \\
--- \\
---\end{array}$ & $\begin{array}{l}9.0 \\
9.0 \\
9.0 \\
9.0 \\
9.0\end{array}$ & $\begin{array}{l}9.5 \\
9.5 \\
9.5 \\
9.5 \\
9.5 \\
9.5\end{array}$ & $\begin{array}{l}10.0 \\
10.0 \\
10.0 \\
10.0 \\
10.0\end{array}$ & $\begin{array}{l}10.5 \\
10.5 \\
10.5 \\
10.5 \\
10.5 \\
10.5\end{array}$ & $\begin{array}{l}11.5 \\
11.5 \\
11.5 \\
11.5 \\
11.0 \\
11.0\end{array}$ & $\begin{array}{l}10.5 \\
10.5 \\
10.5 \\
10.5 \\
10.5\end{array}$ \\
\hline
\end{tabular}


Table 28. Daily mean suspended-sediment concentrations and daily suspended-sediment load at Big Spring, Clayton County, Iowa, water year 1990

[---, data not available to calculate median values]

\begin{tabular}{|c|c|c|c|c|c|c|c|c|c|c|c|c|}
\hline Day & oct. & Nov. & Dec. & Jan. & Feb. & Mar. & Apr. & May & June & July & Aug. & Sept \\
\hline \multicolumn{13}{|c|}{ Daily mean suspended-sediment concentration, in milligrams per liter } \\
\hline $\begin{array}{l}1 \\
2 \\
3 \\
4 \\
5\end{array}$ & $\begin{array}{r}11 \\
11 \\
11 \\
\hdashline--\end{array}$ & $\begin{array}{l}7 \\
6 \\
5 \\
5 \\
4\end{array}$ & $\begin{array}{l}0 \\
0 \\
0 \\
0 \\
0\end{array}$ & $\begin{array}{l}5 \\
5 \\
5 \\
5 \\
6\end{array}$ & $\begin{array}{r}4 \\
4 \\
3 \\
3 \\
18\end{array}$ & $\begin{array}{r}2 \\
3 \\
--2 \\
2 \\
2\end{array}$ & $\begin{array}{r}--- \\
-- \\
5 \\
---\end{array}$ & $\begin{array}{l}8 \\
7 \\
6 \\
6 \\
7\end{array}$ & $\begin{array}{c}9 \\
15 \\
19- \\
17 \\
16\end{array}$ & $\begin{array}{l}24 \\
23 \\
21 \\
19 \\
18\end{array}$ & $\begin{array}{l}11 \\
18 \\
16 \\
23 \\
25\end{array}$ & $\begin{array}{l}38 \\
31 \\
23 \\
20 \\
24\end{array}$ \\
\hline $\begin{array}{r}6 \\
7 \\
8 \\
9 \\
10\end{array}$ & $\begin{array}{c}--- \\
-\because- \\
-\because- \\
10\end{array}$ & $\begin{array}{l}4 \\
3 \\
2 \\
2 \\
1\end{array}$ & $\begin{array}{l}1 \\
1 \\
1 \\
1 \\
1\end{array}$ & $\begin{array}{r}6 \\
5 \\
5 \\
22 \\
20\end{array}$ & $\begin{array}{l}18 \\
19 \\
42 \\
31 \\
23\end{array}$ & $\begin{array}{r}2 \\
2 \\
578 \\
416 \\
146\end{array}$ & $\begin{array}{c}--- \\
--- \\
--- \\
26\end{array}$ & $\begin{array}{r}6 \\
4 \\
3 \\
9 \\
14\end{array}$ & $\begin{array}{l}14 \\
13 \\
12 \\
10 \\
13\end{array}$ & $\begin{array}{l}18 \\
18 \\
20 \\
21 \\
20\end{array}$ & $\begin{array}{r}20 \\
15 \\
12 \\
7 \\
7\end{array}$ & $\begin{array}{l}32 \\
31 \\
28 \\
25 \\
23\end{array}$ \\
\hline $\begin{array}{l}11 \\
12 \\
13 \\
14 \\
15\end{array}$ & $\begin{array}{l}10 \\
10 \\
10 \\
10 \\
10\end{array}$ & $\begin{array}{l}0 \\
0 \\
0 \\
0 \\
0\end{array}$ & $\begin{array}{l}1 \\
1 \\
1 \\
6 \\
3\end{array}$ & $\begin{array}{l}9 \\
3 \\
1 \\
1 \\
3\end{array}$ & $\begin{array}{r}11 \\
8 \\
7 \\
3 \\
2\end{array}$ & $\begin{array}{r}178 \\
112 \\
68 \\
80 \\
73\end{array}$ & $\begin{array}{l}19 \\
12 \\
10 \\
11 \\
11\end{array}$ & $\begin{array}{l}7 \\
7 \\
7 \\
7 \\
6\end{array}$ & $\begin{array}{l}17 \\
23- \\
18 \\
23 \\
23\end{array}$ & $\begin{array}{l}21 \\
22 \\
24 \\
24 \\
21\end{array}$ & $\begin{array}{r}7 \\
10 \\
12 \\
14 \\
17\end{array}$ & $\begin{array}{l}22 \\
21 \\
21 \\
28 \\
25\end{array}$ \\
\hline $\begin{array}{l}16 \\
17 \\
18 \\
19 \\
20\end{array}$ & $\begin{array}{r}10 \\
2 \\
2 \\
7 \\
15\end{array}$ & $\begin{array}{l}0 \\
0 \\
0 \\
1 \\
1\end{array}$ & $\begin{array}{l}4 \\
4 \\
5 \\
6 \\
7\end{array}$ & $\begin{array}{r}17 \\
23 \\
7 \\
2 \\
2\end{array}$ & $\begin{array}{r}2 \\
2 \\
5 \\
3 \\
---\end{array}$ & $\begin{array}{r}33 \\
16 \\
12 \\
10 \\
9\end{array}$ & $\begin{array}{l}12 \\
12 \\
12 \\
12 \\
10\end{array}$ & $\begin{array}{r}9 \\
6 \\
3 \\
9 \\
21\end{array}$ & $\begin{array}{l}16 \\
16 \\
20 \\
19 \\
17\end{array}$ & $\begin{array}{l}18 \\
46 \\
33 \\
32 \\
35\end{array}$ & $\begin{array}{r}21 \\
26 \\
22 \\
36 \\
8\end{array}$ & $\begin{array}{l}19 \\
18 \\
29 \\
42 \\
31\end{array}$ \\
\hline $\begin{array}{l}21 \\
22 \\
23 \\
24 \\
25\end{array}$ & $\begin{array}{l}21 \\
22 \\
22 \\
21 \\
19\end{array}$ & $\begin{array}{l}3 \\
7 \\
6 \\
5 \\
4\end{array}$ & $\begin{array}{r}7 \\
13 \\
6 \\
6 \\
6\end{array}$ & $\begin{array}{l}2 \\
3 \\
3 \\
3 \\
3\end{array}$ & $\begin{array}{l}2 \\
3 \\
3 \\
2 \\
2\end{array}$ & $\begin{array}{r}7 \\
8 \\
5 \\
9 \\
14\end{array}$ & $\begin{array}{r}6 \\
6 \\
8 \\
--19\end{array}$ & $\begin{array}{r}12 \\
-9 \\
10 \\
11\end{array}$ & $\begin{array}{l}11 \\
24 \\
40 \\
38 \\
24\end{array}$ & $\begin{array}{l}28 \\
19 \\
27 \\
34 \\
21\end{array}$ & $\begin{array}{r}22 \\
10 \\
15 \\
1,360 \\
5,100\end{array}$ & $\begin{array}{r}16 \\
11 \\
9 \\
9 \\
14\end{array}$ \\
\hline $\begin{array}{l}26 \\
27 \\
28 \\
29 \\
30 \\
31\end{array}$ & $\begin{array}{r}10 \\
5 \\
5 \\
5 \\
5 \\
10\end{array}$ & $\begin{array}{l}3 \\
3 \\
3 \\
1 \\
0\end{array}$ & $\begin{array}{l}6 \\
6 \\
7 \\
7 \\
6 \\
6\end{array}$ & $\begin{array}{l}2 \\
4 \\
5 \\
4 \\
4 \\
4\end{array}$ & $\begin{array}{l}2 \\
2 \\
2\end{array}$ & $\begin{array}{r}19 \\
18 \\
22 \\
22 \\
--- \\
--\end{array}$ & $\begin{array}{l}29 \\
39 \\
39 \\
26 \\
14\end{array}$ & $\begin{array}{r}10 \\
9 \\
9 \\
13 \\
12 \\
10\end{array}$ & $\begin{array}{l}39 \\
43 \\
33 \\
29 \\
26\end{array}$ & $\begin{array}{l}18 \\
46 \\
40 \\
29 \\
21 \\
16\end{array}$ & $\begin{array}{r}401 \\
105 \\
33 \\
24 \\
16 \\
27\end{array}$ & \begin{tabular}{r}
42 \\
31 \\
--- \\
\hdashline-- \\
--
\end{tabular} \\
\hline
\end{tabular}

Daily suspended-sediment load, in tons

\begin{tabular}{|c|c|c|c|c|c|c|c|c|c|c|c|c|}
\hline $\begin{array}{l}1 \\
2 \\
3 \\
4 \\
5\end{array}$ & $\begin{array}{r}0.37 \\
.36 \\
.36 \\
---\end{array}$ & $\begin{array}{r}0.22 \\
.20 \\
.17 \\
.16 \\
.14\end{array}$ & $\begin{array}{l}0 \\
0 \\
0 \\
0 \\
0\end{array}$ & $\begin{array}{r}0.13 \\
.12 \\
.12 \\
.15 \\
.17\end{array}$ & $\begin{array}{r}0.10 \\
.10 \\
.09 \\
.09 \\
.70\end{array}$ & $\begin{array}{r}0.07 \\
.16 \\
.10 \\
.19\end{array}$ & $\begin{array}{l}-.- \\
.19 \\
.22 \\
--\end{array}$ & $\begin{array}{r}0.35 \\
.27 \\
.23 \\
.28 \\
.27\end{array}$ & $\begin{array}{l}0.52 \\
.87 \\
1.2 \\
1.1 \\
1.0\end{array}$ & $\begin{array}{l}1.5 \\
1.4 \\
1.3 \\
1.1 \\
1.1\end{array}$ & $\begin{array}{l}0.63 \\
1.00 \\
1.89 \\
1.4\end{array}$ & $\begin{array}{l}2.8 \\
2.2 \\
1.6 \\
1.4 \\
1.5\end{array}$ \\
\hline $\begin{array}{r}6 \\
7 \\
8 \\
9 \\
10\end{array}$ & $\begin{array}{l}--- \\
-\because- \\
.- \\
.26\end{array}$ & $\begin{array}{l}.12 \\
.09 \\
.07 \\
.07 \\
.03\end{array}$ & $\begin{array}{l}.01 \\
.02 \\
.03 \\
.03 \\
.04\end{array}$ & $\begin{array}{r}.15 \\
.14 \\
.13 \\
1.0 \\
.88\end{array}$ & $\begin{array}{l}1.2 \\
1.2 \\
2.9 \\
2.0 \\
1.2\end{array}$ & $\begin{array}{c}.18 \\
30.07 \\
24 \\
8.3\end{array}$ & \begin{tabular}{l}
$\cdots$ \\
\hdashline.- \\
\hdashline-1 \\
$-\cdots$
\end{tabular} & $\begin{array}{l}.24 \\
.18 \\
.13 \\
.38 \\
.64\end{array}$ & $\begin{array}{l}.89 \\
.78 \\
.71 \\
.58 \\
.70\end{array}$ & $\begin{array}{l}1.1 \\
1.1 \\
1.2 \\
1.2 \\
1.2\end{array}$ & $\begin{array}{r}1.1 \\
.79 \\
.63 \\
.34 \\
.35\end{array}$ & $\begin{array}{l}1.9 \\
1.9 \\
1.7 \\
1.5 \\
1.4\end{array}$ \\
\hline $\begin{array}{l}11 \\
12 \\
13 \\
14 \\
15\end{array}$ & $\begin{array}{l}.29 \\
.26 \\
.26 \\
.27 \\
.31\end{array}$ & $\begin{array}{l}0 \\
0 \\
0 \\
0 \\
0\end{array}$ & $\begin{array}{l}.04 \\
.02 \\
.02 \\
.17 \\
.08\end{array}$ & $\begin{array}{l}.28 \\
.08 \\
.03 \\
.02 \\
.09\end{array}$ & $\begin{array}{l}.41 \\
.31 \\
.34 \\
.09 \\
.08\end{array}$ & $\begin{array}{r}11 \\
6.7 \\
3.9 \\
5.0 \\
4.3\end{array}$ & $\begin{array}{l}.82 \\
.50 \\
.40 \\
.47 \\
.50\end{array}$ & $\begin{array}{l}.32 \\
.31 \\
.30 \\
.30 \\
.25\end{array}$ & $\begin{array}{l}.91 \\
1.2 \\
1.0 \\
1.3 \\
1.3\end{array}$ & $\begin{array}{l}1.3 \\
1.3 \\
1.4 \\
1.4 \\
1.3\end{array}$ & $\begin{array}{l}.34 \\
.49 \\
.58 \\
.69 \\
.78\end{array}$ & $\begin{array}{l}1.4 \\
1.3 \\
1.1 \\
1.3 \\
1.1\end{array}$ \\
\hline $\begin{array}{l}16 \\
17 \\
18 \\
19 \\
20\end{array}$ & $\begin{array}{l}.32 \\
.08 \\
.06 \\
.24 \\
.45\end{array}$ & $\begin{array}{l}0 \\
0 \\
0 \\
.03 \\
.03\end{array}$ & $\begin{array}{l}.10 \\
.12 \\
.13 \\
.15 \\
.17\end{array}$ & $\begin{array}{r}.51 \\
1.6 \\
.26 \\
.07 \\
.05\end{array}$ & $\begin{array}{l}.07 \\
.08 \\
.15 \\
.09 \\
-.-\end{array}$ & $\begin{array}{r}2.0 \\
.96 \\
.71 \\
.55 \\
.51\end{array}$ & $\begin{array}{l}.50 \\
.50 \\
.49 \\
.47 \\
.44\end{array}$ & $\begin{array}{r}.37 \\
.27 \\
.12 \\
.50 \\
1.4\end{array}$ & $\begin{array}{l}.97 \\
.97 \\
1.2 \\
1.2 \\
1.1\end{array}$ & $\begin{array}{l}1.0 \\
2.5 \\
1.9 \\
1.9 \\
2.1\end{array}$ & $\begin{array}{c}.95 \\
1.3 \\
1.1 \\
2.2 \\
.51\end{array}$ & $\begin{array}{l}.82 \\
.76 \\
1.3 \\
1.8 \\
1.3\end{array}$ \\
\hline $\begin{array}{l}21 \\
22 \\
23 \\
24 \\
25\end{array}$ & $\begin{array}{l}.62 \\
.66 \\
.66 \\
.63 \\
.56\end{array}$ & $\begin{array}{l}.08 \\
.20 \\
.17 \\
.15 \\
.12\end{array}$ & $\begin{array}{l}.18 \\
.33 \\
.14 \\
.15 \\
.15\end{array}$ & $\begin{array}{l}.06 \\
.08 \\
.08 \\
.08 \\
.07\end{array}$ & $\begin{array}{l}.07 \\
.08 \\
.08 \\
.07 \\
.06\end{array}$ & $\begin{array}{l}.37 \\
.46 \\
.27 \\
.46 \\
.70\end{array}$ & $\begin{array}{r}.26 \\
.25 \\
.37 \\
-.89\end{array}$ & $\begin{array}{l}.75 \\
-.56 \\
.65 \\
.69\end{array}$ & $\begin{array}{l}.70 \\
1.6 \\
2.6 \\
2.5 \\
1.5\end{array}$ & $\begin{array}{l}1.6 \\
1.2 \\
1.6 \\
1.9 \\
1.2\end{array}$ & $\begin{array}{r}1.3 \\
.61 \\
.81 \\
275\end{array}$ & $\begin{array}{l}.68 \\
.47 \\
.40 \\
.36 \\
.55\end{array}$ \\
\hline $\begin{array}{l}26 \\
27 \\
28 \\
29 \\
30 \\
31\end{array}$ & $\begin{array}{l}.29 \\
.16 \\
.15 \\
.14 \\
.15 \\
.32\end{array}$ & $\begin{array}{r}.09 \\
.10 \\
.08 \\
.04\end{array}$ & $\begin{array}{l}.15 \\
.16 \\
.17 \\
.17 \\
.16 \\
.15\end{array}$ & $\begin{array}{l}.06 \\
.11 \\
.12 \\
.12 \\
.11 \\
.09\end{array}$ & $\begin{array}{l}.05 \\
.05 \\
.05\end{array}$ & $\begin{array}{r}.92 \\
.88 \\
1.0 \\
.99 \\
-.-\end{array}$ & $\begin{array}{c}1.3 \\
1.8 \\
1.8 \\
1.2 \\
.59\end{array}$ & $\begin{array}{l}.65 \\
.53 \\
.59 \\
.80 \\
.70 \\
.59\end{array}$ & $\begin{array}{l}2.6 \\
2.7 \\
2.0 \\
1.8 \\
1.6\end{array}$ & $\begin{array}{l}.96 \\
2.7 \\
2.4 \\
1.7 \\
1.3 \\
.96\end{array}$ & $\begin{array}{l}12 \\
3.1 \\
2.2 \\
1.8 \\
1.2 \\
1.9\end{array}$ & \begin{tabular}{c}
1.6 \\
1.3 \\
\hdashline.- \\
\hdashline--
\end{tabular} \\
\hline $\begin{array}{l}\text { otal } \\
\text { easured }\end{array}$ & 8.23 & 2.36 & 3.04 & 6.96 & 11.7 & 104 & 15.1 & 13.6 & 39.1 & 45.8 & 398 & 35.4 \\
\hline
\end{tabular}


Table 29. Daily mean water levels in the Saint Peter aquifer, Clayton County, Iowa, water year 1990

[Water levels in feet below land surface; --, data not available to calculate mean values]

Day Oct. Nov. Dec. Jan. Feb. Mar. Apr. May June July Aug. Sept.

\begin{tabular}{|c|c|c|c|c|c|c|c|c|c|c|c|c|}
\hline \multicolumn{13}{|c|}{ Well BS2-G (fig, 2) } \\
\hline $\begin{array}{l}1 \\
2 \\
3 \\
4 \\
5\end{array}$ & $\begin{array}{l}185.03 \\
185.18 \\
185.42 \\
185.56 \\
185.73\end{array}$ & $\begin{array}{l}185.39 \\
185.45 \\
185.36 \\
185.08 \\
184.99\end{array}$ & $\begin{array}{l}185.48 \\
185.56 \\
185.62 \\
185.18 \\
185.13\end{array}$ & $\begin{array}{l}185.65 \\
185.58 \\
185.52 \\
185.60 \\
185.65\end{array}$ & $\begin{array}{l}185.63 \\
185.73 \\
185.74 \\
185.77 \\
185.66\end{array}$ & $\begin{array}{l}186.00 \\
185.74 \\
185.90 \\
185.96 \\
185.97\end{array}$ & $\begin{array}{r}185.56 \\
185.85 \\
185.80 \\
185.54\end{array}$ & $\begin{array}{r}185.31 \\
-185.91 \\
185.72 \\
185.65\end{array}$ & $\begin{array}{r}185.54 \\
185.37 \\
185.38 \\
185.58 \\
-\end{array}$ & $\begin{array}{l}185.50 \\
185.48 \\
185.32 \\
185.53 \\
185.63\end{array}$ & $\begin{array}{l}185.75 \\
185.62 \\
185.50 \\
185.54 \\
185.82\end{array}$ & $\begin{array}{l}185.64 \\
185.69 \\
185.69 \\
185.68 \\
185.58\end{array}$ \\
\hline $\begin{array}{r}6 \\
7 \\
8 \\
9 \\
10\end{array}$ & $\begin{array}{l}185.38 \\
185.39 \\
185.30 \\
185.21 \\
185.15\end{array}$ & $\begin{array}{l}185.25 \\
185.14 \\
184.97 \\
184.91 \\
185.13\end{array}$ & $\begin{array}{l}185.36 \\
185.72 \\
185.65 \\
185.41 \\
185.46\end{array}$ & $\begin{array}{l}185.69 \\
185.56 \\
185.37 \\
185.38 \\
185.47\end{array}$ & $\begin{array}{l}185.71 \\
185.76 \\
185.57 \\
185.68 \\
185.77\end{array}$ & $\begin{array}{l}186.14 \\
186.21 \\
185.81 \\
185.71 \\
185.73\end{array}$ & $\begin{array}{r}185.79 \\
185.70 \\
-\end{array}$ & $\begin{array}{l}185.61 \\
185.48 \\
185.52 \\
185.44 \\
185.48\end{array}$ & $\begin{array}{l}185.41 \\
185.52 \\
185.43 \\
185.51 \\
185.65\end{array}$ & $\begin{array}{l}185.67 \\
185.53 \\
185.44 \\
185.53 \\
185.72\end{array}$ & $\begin{array}{l}185.81 \\
185.74 \\
185.66 \\
185.67 \\
185.63\end{array}$ & $\begin{array}{l}185.32 \\
185.33 \\
185.57 \\
185.43 \\
185.42\end{array}$ \\
\hline $\begin{array}{l}11 \\
12 \\
13 \\
14 \\
15\end{array}$ & $\begin{array}{l}185.01 \\
185.21 \\
185.24 \\
185.24 \\
185.36\end{array}$ & $\begin{array}{l}185.35 \\
185.45 \\
185.15 \\
185.18 \\
185.18\end{array}$ & $\begin{array}{l}185.59 \\
185.73 \\
185.48 \\
185.52 \\
185.58\end{array}$ & $\begin{array}{l}185.35 \\
185.69 \\
185.82 \\
185.51 \\
185.60\end{array}$ & $\begin{array}{l}185.77 \\
185.60 \\
185.67 \\
185.95 \\
185.67\end{array}$ & $\begin{array}{l}185.58 \\
185.59 \\
185.73 \\
185.41 \\
185.31\end{array}$ & $\begin{array}{r}185.71 \\
185.83 \\
\hdashline-\end{array}$ & $\begin{array}{l}185.63 \\
185.58 \\
185.66 \\
185.60 \\
185.54\end{array}$ & $\begin{array}{l}185.68 \\
185.46 \\
185.39 \\
185.50 \\
185.56\end{array}$ & $\begin{array}{l}185.59 \\
185.73 \\
185.80 \\
185.54 \\
185.53\end{array}$ & $\begin{array}{l}185.80 \\
185.70 \\
185.74 \\
185.73 \\
185.73\end{array}$ & $\begin{array}{l}185.42 \\
185.41 \\
185.43 \\
185.42 \\
185.43\end{array}$ \\
\hline $\begin{array}{l}16 \\
17 \\
18 \\
19 \\
20\end{array}$ & $\begin{array}{l}185.28 \\
185.50 \\
185.68 \\
185.52 \\
185.24\end{array}$ & $\begin{array}{l}185.32 \\
185.16 \\
185.46 \\
185.36 \\
185.28\end{array}$ & $\begin{array}{l}185.63 \\
185.58 \\
185.74 \\
185.78 \\
185.75\end{array}$ & $\begin{array}{l}185.63 \\
185.68 \\
185.95 \\
185.90 \\
185.60\end{array}$ & $\begin{array}{l}185.72 \\
186.04 \\
185.83 \\
186.04 \\
186.13\end{array}$ & $\begin{array}{l}185.45 \\
185.59 \\
185.76 \\
185.98 \\
185.96\end{array}$ & $\begin{array}{r}185.63 \\
185.66 \\
185.80 \\
185.92\end{array}$ & $\begin{array}{l}185.44 \\
185.67 \\
185.66 \\
185.37 \\
185.47\end{array}$ & $\begin{array}{l}185.48 \\
185.36 \\
185.47 \\
185.41 \\
185.34\end{array}$ & $\begin{array}{l}185.52 \\
185.54 \\
185.54 \\
185.51 \\
185.51\end{array}$ & $\begin{array}{l}185.73 \\
185.69 \\
185.73 \\
185.66 \\
185.44\end{array}$ & $\begin{array}{l}185.44 \\
185.45 \\
185.43 \\
185.42 \\
185.43\end{array}$ \\
\hline $\begin{array}{l}21 \\
22 \\
23 \\
24 \\
25\end{array}$ & $\begin{array}{l}185.30 \\
185.34 \\
185.34 \\
185.41 \\
185.43\end{array}$ & $\begin{array}{l}185.40 \\
185.64 \\
185.52 \\
185.29 \\
185.19\end{array}$ & $\begin{array}{l}185.98 \\
186.11 \\
186.05 \\
185.82 \\
185.44\end{array}$ & $\begin{array}{l}185.57 \\
185.60 \\
185.32 \\
185.41 \\
185.41\end{array}$ & $\begin{array}{l}185.84 \\
185.59 \\
185.57 \\
185.87 \\
186.22\end{array}$ & $\begin{array}{l}185.77 \\
185.78 \\
186.09 \\
186.07 \\
185.98\end{array}$ & $\begin{array}{l}185.76 \\
185.77 \\
185.75\end{array}$ & $\begin{array}{l}185.64 \\
185.66 \\
185.58 \\
185.57 \\
185.52\end{array}$ & $\begin{array}{l}185.46 \\
185.27 \\
185.33 \\
185.43 \\
185.49\end{array}$ & $\begin{array}{l}185.63 \\
185.50 \\
185.52 \\
185.51 \\
185.55\end{array}$ & $\begin{array}{l}185.23 \\
185.67 \\
185.73 \\
185.69 \\
185.69\end{array}$ & $\begin{array}{l}185.43 \\
185.43 \\
185.43 \\
185.43 \\
185.43\end{array}$ \\
\hline $\begin{array}{l}26 \\
27 \\
28 \\
29 \\
30 \\
31\end{array}$ & $\begin{array}{l}185.42 \\
185.39 \\
185.38 \\
185.30 \\
185.38 \\
185.26\end{array}$ & $\begin{array}{l}185.35 \\
185.29 \\
185.58 \\
185.73 \\
185.58\end{array}$ & $\begin{array}{l}185.59 \\
185.52 \\
185.50 \\
185.56 \\
185.57 \\
185.46\end{array}$ & $\begin{array}{l}185.47 \\
185.47 \\
185.75 \\
185.49 \\
185.50 \\
185.63\end{array}$ & $\begin{array}{l}186.04 \\
186.08 \\
186.31\end{array}$ & $\begin{array}{l}186.06 \\
186.14 \\
185.94 \\
185.83 \\
185.76 \\
185.67\end{array}$ & $\begin{array}{r}185.51 \\
185.50 \\
185.65 \\
185.44\end{array}$ & $\begin{array}{l}185.60 \\
185.53 \\
185.48 \\
185.54 \\
185.62 \\
185.58\end{array}$ & $\begin{array}{l}185.50 \\
185.47 \\
185.44 \\
185.43 \\
185.43\end{array}$ & $\begin{array}{l}185.62 \\
185.58 \\
185.47 \\
185.37 \\
185.45 \\
185.56\end{array}$ & $\begin{array}{l}185.69 \\
185.71 \\
185.56 \\
185.73 \\
185.48 \\
185.70\end{array}$ & $\begin{array}{l}185.43 \\
185.43 \\
185.43 \\
185.43 \\
185.43\end{array}$ \\
\hline \multicolumn{13}{|c|}{ Well BS4 (fig. 2) } \\
\hline $\begin{array}{l}1 \\
2 \\
3 \\
4 \\
5\end{array}$ & $\begin{array}{l}370.12 \\
370.38 \\
370.57 \\
370.55 \\
370.14\end{array}$ & $\begin{array}{l}370.55 \\
370.60 \\
370.46 \\
370.19 \\
370.09\end{array}$ & $\begin{array}{l}369.99 \\
370.02 \\
370.16 \\
369.64 \\
369.66\end{array}$ & $\begin{array}{l}369.78 \\
369.58 \\
369.40 \\
369.41 \\
369.40\end{array}$ & $\begin{array}{l}369.11 \\
369.27 \\
369.28 \\
369.27 \\
369.19\end{array}$ & $\begin{array}{l}369.62 \\
369.37 \\
369.50 \\
369.39 \\
369.53\end{array}$ & $\begin{array}{l}368.91 \\
369.10 \\
369.14 \\
368.88 \\
368.98\end{array}$ & $\begin{array}{l}368.95 \\
369.04 \\
369.08 \\
368.88 \\
368.85\end{array}$ & $\begin{array}{l}369.43 \\
369.15 \\
369.27 \\
369.57 \\
369.37\end{array}$ & $\begin{array}{l}370.28 \\
370.28 \\
370.18 \\
370.27 \\
370.47\end{array}$ & $\begin{array}{l}371.19 \\
371.13 \\
371.05 \\
371.04 \\
371.25\end{array}$ & 371.69 \\
\hline $\begin{array}{r}6 \\
7 \\
8 \\
9 \\
10\end{array}$ & $\begin{array}{l}370.28 \\
370.45 \\
370.40 \\
370.31 \\
370.27\end{array}$ & $\begin{array}{l}370.42 \\
370.31 \\
370.17 \\
370.09 \\
370.30\end{array}$ & $\begin{array}{l}369.97 \\
370.36 \\
370.28 \\
369.93 \\
370.08\end{array}$ & $\begin{array}{l}369.37 \\
369.13 \\
368.83 \\
368.87 \\
368.94\end{array}$ & $\begin{array}{l}369.27 \\
369.26 \\
369.04 \\
369.11 \\
369.15\end{array}$ & $\begin{array}{l}369.73 \\
369.72 \\
369.34 \\
369.27 \\
369.23\end{array}$ & $\begin{array}{l}369.11 \\
369.20 \\
369.14 \\
369.06 \\
369.09\end{array}$ & $\begin{array}{l}368.83 \\
368.70 \\
368.72 \\
368.60 \\
368.72\end{array}$ & $\begin{array}{l}369.43 \\
369.49 \\
369.42 \\
369.55 \\
369.75\end{array}$ & $\begin{array}{l}370.56 \\
370.45 \\
370.38 \\
370.52 \\
370.56\end{array}$ & $\begin{array}{l}371.40 \\
371.36 \\
371.29 \\
371.26 \\
371.23\end{array}$ & $\begin{array}{l}--- \\
--- \\
---\end{array}$ \\
\hline $\begin{array}{l}11 \\
12 \\
13 \\
14 \\
15\end{array}$ & $\begin{array}{l}370.12 \\
370.33 \\
370.33 \\
370.23 \\
370.13\end{array}$ & $\begin{array}{l}370.28 \\
370.45 \\
370.14 \\
370.18 \\
370.16\end{array}$ & $\begin{array}{l}370.30 \\
370.21 \\
370.10 \\
370.21 \\
370.29\end{array}$ & $\begin{array}{l}368.77 \\
369.12 \\
369.14 \\
368.83 \\
368.93\end{array}$ & $\begin{array}{l}369.24 \\
369.10 \\
369.24 \\
369.54 \\
369.24\end{array}$ & $\begin{array}{l}369.08 \\
368.96 \\
369.04 \\
368.76 \\
368.64\end{array}$ & $\begin{array}{l}369.23 \\
369.20 \\
369.10 \\
368.94 \\
368.92\end{array}$ & $\begin{array}{l}368.96 \\
368.94 \\
369.10 \\
369.07 \\
369.06\end{array}$ & $\begin{array}{l}369.70 \\
369.48 \\
369.49 \\
369.65 \\
369.67\end{array}$ & $\begin{array}{l}370.53 \\
370.65 \\
370.73 \\
370.54 \\
370.49\end{array}$ & $\begin{array}{r}371.26 \\
371.31 \\
\hdashline-. \\
\hdashline-\end{array}$ & 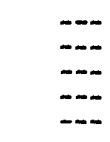 \\
\hline $\begin{array}{l}16 \\
17 \\
18 \\
19 \\
20\end{array}$ & $\begin{array}{l}370.32 \\
370.59 \\
370.79 \\
370.59 \\
370.24\end{array}$ & $\begin{array}{l}370.21 \\
370.04 \\
370.29 \\
370.08 \\
370.05\end{array}$ & $\begin{array}{l}370.36 \\
370.31 \\
370.45 \\
370.48 \\
370.51\end{array}$ & $\begin{array}{l}368.94 \\
368.86 \\
369.18 \\
369.17 \\
368.84\end{array}$ & $\begin{array}{l}369.15 \\
369.60 \\
369.40 \\
369.66 \\
369.74\end{array}$ & $\begin{array}{l}368.77 \\
368.80 \\
369.03 \\
369.33 \\
369.29\end{array}$ & $\begin{array}{l}368.85 \\
369.11 \\
369.17 \\
368.92 \\
368.84\end{array}$ & $\begin{array}{l}368.94 \\
369.07 \\
369.25 \\
369.01 \\
369.19\end{array}$ & $\begin{array}{r}369.58 \\
369.56 \\
369.75 \\
\hdashline-2\end{array}$ & $\begin{array}{l}370.61 \\
370.71 \\
370.78 \\
370.74 \\
370.72\end{array}$ & 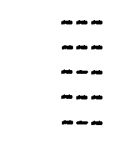 & 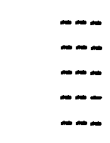 \\
\hline $\begin{array}{l}21 \\
22 \\
23 \\
24 \\
25\end{array}$ & $\begin{array}{l}370.24 \\
370.39 \\
370.43 \\
370.48 \\
370.47\end{array}$ & $\begin{array}{l}370.21 \\
370.19 \\
370.17 \\
369.91 \\
369.85\end{array}$ & $\begin{array}{l}370.75 \\
370.91 \\
370.74 \\
370.34 \\
370.04\end{array}$ & $\begin{array}{l}368.79 \\
368.84 \\
368.61 \\
368.78 \\
368.78\end{array}$ & $\begin{array}{l}369.45 \\
369.19 \\
369.18 \\
369.51 \\
369.92\end{array}$ & $\begin{array}{l}368.99 \\
368.95 \\
369.39 \\
369.35 \\
369.21\end{array}$ & $\begin{array}{l}368.92 \\
368.83 \\
368.67 \\
368.63 \\
368.72\end{array}$ & $\begin{array}{l}369.43 \\
369.50 \\
369.42 \\
369.43 \\
369.35\end{array}$ & 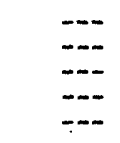 & $\begin{array}{l}370.79 \\
370.80 \\
370.86 \\
370.93 \\
370.99\end{array}$ & 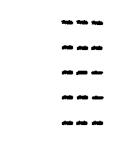 & $\begin{array}{l}--- \\
--- \\
--- \\
--\end{array}$ \\
\hline $\begin{array}{l}26 \\
27 \\
28 \\
29 \\
30 \\
31\end{array}$ & $\begin{array}{l}370.47 \\
370.45 \\
370.44 \\
370.38 \\
370.33 \\
370.37\end{array}$ & $\begin{array}{l}369.98 \\
369.66 \\
370.16 \\
370.14 \\
370.05\end{array}$ & $\begin{array}{l}370.17 \\
369.96 \\
369.95 \\
369.95 \\
369.83 \\
369.60\end{array}$ & $\begin{array}{l}368.85 \\
368.85 \\
369.18 \\
368.94 \\
369.02 \\
369.15\end{array}$ & $\begin{array}{l}369.69 \\
369.79 \\
369.89\end{array}$ & $\begin{array}{l}369.35 \\
369.31 \\
369.22 \\
369.16 \\
369.11 \\
369.04\end{array}$ & $\begin{array}{l}368.65 \\
368.54 \\
368.45 \\
368.62 \\
368.84\end{array}$ & $\begin{array}{l}369.40 \\
369.44 \\
369.47 \\
369.54 \\
369.63 \\
369.59\end{array}$ & 370.19 & $\begin{array}{l}371.03 \\
370.99 \\
370.94 \\
370.91 \\
371.06 \\
371.19\end{array}$ & 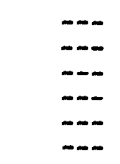 & $\begin{array}{l}--- \\
--- \\
--- \\
--\end{array}$ \\
\hline
\end{tabular}


Table 30. Onsite determinations and chemical analyses of samples from streams in the Big Spring basin during low-flow conditions, May 29-30, 1990

[ft ${ }^{3} / \mathrm{s}$, cubic feet per second: $\mu \mathrm{S} / \mathrm{cm}$, microsiemens per centimeter at 25 degrees Celsius; ${ }^{\circ} \mathrm{C}$, degrees Celsius; $\mathrm{N}$, nitrogen; $\mathrm{P}$, phosphorus; $\mathrm{C}$, carbon; <, less than; --, missing data]

\begin{tabular}{|c|c|c|c|c|c|c|c|c|c|c|c|}
\hline $\begin{array}{l}\text { Site } \\
\text { desig- } \\
\text { nation }\end{array}$ & Date & Time & $\begin{array}{l}\text { Dis- } \\
\text { charge }\end{array}$ & $\begin{array}{l}\text { Spe- } \\
\text { cific } \\
\text { con- } \\
\text { duct- } \\
\text { ance }\end{array}$ & $\begin{array}{c}\mathrm{pH} \\
\text { (standard } \\
\text { units) }\end{array}$ & $\begin{array}{l}\text { Water } \\
\text { temper- } \\
\text { ature } \\
\text { ('C) }\end{array}$ & $\begin{array}{l}\text { Mitrite } \\
\text { plus } \\
\text { nitrate, } \\
\text { dis- } \\
\text { solved } \\
\text { (m8/L } \\
\text { as } N \text { ) }\end{array}$ & $\begin{array}{l}\text { Ammonia, } \\
\text { dis- } \\
\text { solved - } \\
\text { (mg/L } / L \text {. } \\
\text { as } N \text {. }\end{array}$ & $\begin{array}{l}\text { Organic } \\
\text { nitrogen, } \\
\text { dis- } \\
\text { solved } \\
\text { (mg/L } \\
\text { as N) }\end{array}$ & $\begin{array}{l}\text { Ortho- } \\
\text { phos- } \\
\text { phorus, } \\
\text { dis- } \\
\text { solved } \\
\text { (mg/L } \\
\text { as P) }\end{array}$ & $\begin{array}{l}\text { Total } \\
\text { organic } \\
\text { carbon } \\
\text { mg/L } \\
\text { (as C) }\end{array}$ \\
\hline $\begin{array}{l}\text { BS5 } \\
\text { BS6 } \\
\text { BS4 } \\
\text { BS2 } \\
\text { BS3 }\end{array}$ & $\begin{array}{l}5-30-90 \\
5-30-90 \\
5-30-90 \\
5-30-90 \\
5-30-90\end{array}$ & $\begin{array}{l}0710 \\
0840 \\
0930 \\
1020 \\
1100\end{array}$ & $\begin{array}{r}0.20 \\
.61 \\
.61 \\
.60 \\
1.55\end{array}$ & $\begin{array}{l}713 \\
688 \\
700 \\
693 \\
655\end{array}$ & $\begin{array}{l}8.0 \\
7.8 \\
7.8 \\
8.2 \\
8.4\end{array}$ & $\begin{array}{l}10.0 \\
10.5 \\
11.0 \\
11.0 \\
15.0\end{array}$ & $\begin{array}{c}5.5 \\
4.5 \\
10 \\
9.4 \\
7.7\end{array}$ & $\begin{array}{l}<0.10 \\
<.10 \\
<.10 \\
<.10 \\
<.10\end{array}$ & $\begin{array}{r}0.50 \\
.40 \\
.20 \\
.40 \\
.40\end{array}$ & $\begin{array}{r}0.20 \\
.10 \\
\times .10 \\
.20 \\
.10\end{array}$ & $\begin{array}{l}4.3 \\
3.3 \\
2.2 \\
2.7 \\
2.9\end{array}$ \\
\hline $\begin{array}{l}\mathrm{BS1} \\
\mathrm{RC13} \\
\mathrm{RC26} \\
\mathrm{RC25} \\
\mathrm{RC21}\end{array}$ & $\begin{array}{l}5-30-90 \\
5-30-90 \\
5-30-90 \\
5-30-90 \\
5-30-90\end{array}$ & $\begin{array}{l}-\overline{1100} \\
1305 \\
0820 \\
1600\end{array}$ & $\begin{array}{r}0 \\
.35 \\
.95 \\
.12 \\
1.07\end{array}$ & $\begin{array}{l}954 \\
630 \\
798 \\
644\end{array}$ & $\begin{array}{l}-. \overline{7} \\
8.0 \\
7.5 \\
8.2\end{array}$ & $\begin{array}{l}15.5 \\
21.5 \\
10.5 \\
22.5\end{array}$ & $\begin{array}{c}-18 \\
18 \\
4.9 \\
7.4 \\
6.8\end{array}$ & $\begin{array}{l}2.0 \\
<.10 \\
<.10 \\
<.10\end{array}$ & $\begin{array}{l}1.6 \\
.20 \\
.60 \\
.60\end{array}$ & $\begin{array}{r}-.90 \\
.90 \\
.50 \\
<.10 \\
.10\end{array}$ & $\begin{array}{l}6 . \overline{-} \\
2.0 \\
2.8 \\
4.2\end{array}$ \\
\hline $\begin{array}{l}\text { RC20 } \\
\text { RC11 } \\
\text { RC10 } \\
\text { RC15 } \\
\text { DC2 }\end{array}$ & $\begin{array}{l}5-30-90 \\
5-30-90 \\
5-30-90 \\
5-30-90 \\
5-30-90\end{array}$ & $\begin{array}{l}1500 \\
1655 \\
1620 \\
1635 \\
1515\end{array}$ & $\begin{array}{r}2.76 \\
3.42 \\
3.61 \\
3.96 \\
.43\end{array}$ & $\begin{array}{l}711 \\
675 \\
625 \\
680 \\
670\end{array}$ & $\begin{array}{l}8.0 \\
8.3 \\
8.9 \\
7.5 \\
7.3\end{array}$ & $\begin{array}{l}21.5 \\
22.0 \\
22.0 \\
21.0 \\
20.0\end{array}$ & $\begin{array}{l}8.1 \\
7.0 \\
6.6 \\
5.3 \\
1.9\end{array}$ & $\begin{array}{r}<.10 \\
<.10 \\
<.10 \\
.10 \\
.20\end{array}$ & $\begin{array}{l}.60 \\
.50 \\
.60 \\
.70 \\
.80\end{array}$ & $\begin{array}{l}.20 \\
.20 \\
.10 \\
.20 \\
.50\end{array}$ & $\begin{array}{l}3.6 \\
3.7 \\
4.5 \\
4.6 \\
4.2\end{array}$ \\
\hline $\begin{array}{l}\mathrm{F} 45 \\
\mathrm{RC} 29 \\
\mathrm{RC} 16 \\
\mathrm{RC} 17 \\
\mathrm{SC} 10\end{array}$ & $\begin{array}{l}5-30-90 \\
5-30-90 \\
5-30-90 \\
5-30-90 \\
5-30-90\end{array}$ & $\begin{array}{l}1255 \\
1130 \\
0950 \\
0800 \\
0845\end{array}$ & $\begin{array}{r}5.54 \\
.47 \\
6.28 \\
7.09 \\
.42\end{array}$ & $\begin{array}{r}690 \\
660 \\
695 \\
680 \\
1,020\end{array}$ & $\begin{array}{l}7.3 \\
7.4 \\
7.6 \\
7.4 \\
8.0\end{array}$ & $\begin{array}{l}18.0 \\
15.0 \\
17.0 \\
16.0 \\
11.0\end{array}$ & $\begin{array}{l}5.5 \\
6.4 \\
6.4 \\
6.4 \\
15\end{array}$ & $\begin{array}{r}.20 \\
<.10 \\
.20 \\
.20 \\
.20\end{array}$ & $\begin{array}{l}.70 \\
.30 \\
.80 \\
.80 \\
.90\end{array}$ & $\begin{array}{l}.20 \\
.10 \\
.20 \\
.20 \\
2.0\end{array}$ & $\begin{array}{l}4.3 \\
2.4 \\
4.8 \\
4.9 \\
3.6\end{array}$ \\
\hline $\begin{array}{l}\text { SC13 } \\
\text { SC3 } \\
\text { SC6 } \\
\text { SCU1 } \\
\text { SCU2 }\end{array}$ & $\begin{array}{l}5-30-90 \\
5-30-90 \\
5-30-90 \\
5-30-90 \\
5-30-90\end{array}$ & $\begin{array}{l}0720 \\
1030 \\
1600 \\
1250 \\
1230\end{array}$ & $\begin{array}{l}.95 \\
.90 \\
.36 \\
.14 \\
.02\end{array}$ & $\begin{array}{r}1,000 \\
1,250 \\
1,180 \\
890 \\
713\end{array}$ & $\begin{array}{l}8.0 \\
8.4 \\
8.3 \\
8.2 \\
8.0\end{array}$ & $\begin{array}{l}28.0 \\
18.0 \\
22.0 \\
23.0 \\
18.0\end{array}$ & $\begin{array}{l}2.4 \\
6.0 \\
6.2 \\
2.6 \\
5.6\end{array}$ & $\begin{array}{r}.70 \\
.70 \\
.50 \\
<.10 \\
<.10\end{array}$ & $\begin{array}{l}.60 \\
1.0 \\
1.3 \\
.60 \\
.30\end{array}$ & $\begin{array}{r}7.2 \\
7.0 \\
4.4 \\
<.10 \\
<.10\end{array}$ & $\begin{array}{r}3.7 \\
4.6 \\
8.6 \\
5.2 \\
. \quad 2.2\end{array}$ \\
\hline $\begin{array}{l}\text { L23S } \\
\text { SC5 } \\
\text { SC1 } \\
\text { SC14 } \\
\text { SC2 }\end{array}$ & $\begin{array}{l}5-30-90 \\
5-30-90 \\
5-30-90 \\
5-30-90 \\
5-29-90\end{array}$ & $\begin{array}{l}1420 \\
1300 \\
1525 \\
0955 \\
1715\end{array}$ & $\begin{array}{r}.56 \\
1.13 \\
.70 \\
.23 \\
.80\end{array}$ & $\begin{array}{l}720 \\
600 \\
855 \\
720 \\
770\end{array}$ & $\begin{array}{l}8.7 \\
8.4 \\
8.7 \\
8.1 \\
8.3\end{array}$ & $\begin{array}{l}23.0 \\
17.0 \\
22.0 \\
11.0 \\
27.0\end{array}$ & $\begin{array}{l}6.4 \\
7.6 \\
6.8 \\
6.7 \\
4.7\end{array}$ & $\begin{array}{r}.10 \\
<.10 \\
.10 \\
<.10 \\
.40\end{array}$ & $\begin{array}{l}1.7 \\
.70 \\
1.0 \\
.60 \\
2.1\end{array}$ & $\begin{array}{r}.20 \\
.20 \\
1.1 \\
.10 \\
1.0\end{array}$ & $\begin{array}{l}7.4 \\
4.3 \\
7.0 \\
3.1 \\
8.8\end{array}$ \\
\hline $\begin{array}{l}\mathrm{SC}_{4} \\
\mathrm{RC} 18 \\
\mathrm{RC} 19 \\
\mathrm{~F} 47 \\
\mathrm{RC} 24\end{array}$ & $\begin{array}{l}5-29-90 \\
5-30-90 \\
5-30-90 \\
5-30-90 \\
5-30-90\end{array}$ & $\begin{array}{l}1550 \\
0755 \\
0945 \\
1135 \\
1325\end{array}$ & $\begin{array}{l}1.30 \\
7.33 \\
6.72 \\
6.38 \\
5.93\end{array}$ & $\begin{array}{l}890 \\
706 \\
713 \\
712 \\
663\end{array}$ & $\begin{array}{l}8.2 \\
8.0 \\
8.2 \\
8.2 \\
8.8\end{array}$ & $\begin{array}{l}23.0 \\
15.5 \\
17.0 \\
18.0 \\
20.5\end{array}$ & $\begin{array}{l}4.8 \\
5.7 \\
5.6 \\
5.5 \\
5.0\end{array}$ & $\begin{array}{r}.40 \\
.20 \\
.10 \\
<.10 \\
<.10\end{array}$ & $\begin{array}{l}1.3 \\
1.0 \\
.70 \\
.80 \\
.70\end{array}$ & $\begin{array}{r}1.7 \\
.30 \\
.20 \\
.30 \\
.20\end{array}$ & $\begin{array}{l}9.0 \\
6.3 \\
4.8 \\
4.7 \\
6.3\end{array}$ \\
\hline $\begin{array}{l}\mathrm{RC22} \\
\mathrm{RC2} \\
\mathrm{BC} 1 \\
\mathrm{BC2}\end{array}$ & $\begin{array}{l}5-30-90 \\
5-30-90 \\
5-29-90 \\
5-29-90\end{array}$ & $\begin{array}{l}1515 \\
1655 \\
1520 \\
1710\end{array}$ & $\begin{array}{r}3.85 \\
3.29 \\
.32 \\
.34\end{array}$ & $\begin{array}{l}640 \\
621 \\
676 \\
602\end{array}$ & $\begin{array}{l}8.9 \\
8.8 \\
8.4 \\
8.3\end{array}$ & $\begin{array}{l}23.0 \\
24.0 \\
21.5 \\
23.0\end{array}$ & $\begin{array}{l}4.7 \\
4.0 \\
1.7 \\
1.7\end{array}$ & $\begin{array}{l}<.10 \\
<.10 \\
<.10 \\
<.10\end{array}$ & $\begin{array}{l}.70 \\
.50 \\
.40 \\
.60\end{array}$ & $\begin{array}{l}.20 \\
.10 \\
.10 \\
.20\end{array}$ & $\begin{array}{l}5.2 \\
5.0 \\
3.2 \\
4.1\end{array}$ \\
\hline
\end{tabular}


Table 31. Selected pesticides in samples from streams in the Big Spring basin during low-flow conditions, May 29-30, 1990

[Total-recoverable constituents in micrograms per liter; <, less than; --, data not collected]

\begin{tabular}{|c|c|c|c|c|c|c|c|c|c|}
\hline \multicolumn{2}{|c|}{$\begin{array}{l}\text { Site } \\
\text { desig- } \\
\text { nation Date } \\
(\text { fig. 16) }\end{array}$} & (24-hour) & $\begin{array}{l}\text { Ala- } \\
\text { chlor }\end{array}$ & $\begin{array}{l}\text { Atra- } \\
\text { zine }\end{array}$ & $\begin{array}{l}\text { Buty- } \\
\text { late }\end{array}$ & $\begin{array}{c}\text { Cyana- } \\
\text { zine }\end{array}$ & $\begin{array}{l}\text { Metola- } \\
\text { chlor }\end{array}$ & $\begin{array}{l}\text { Metri- } \\
\text { buzin }\end{array}$ & $\begin{array}{l}\text { Tri- } \\
\text { flur- } \\
\text { alin }\end{array}$ \\
\hline $\begin{array}{l}\text { ES5 } \\
\text { ES6 } \\
\text { ES4 } \\
\text { ES2 } \\
\text { BS3 }\end{array}$ & $\begin{array}{l}5-30-90 \\
5-30-90 \\
5-30-90 \\
5-30-90 \\
5-30-90\end{array}$ & $\begin{array}{l}0710 \\
0840 \\
0930 \\
1020 \\
1110\end{array}$ & $\begin{array}{l}<- \\
<0.10 \\
<.10 \\
<.10 \\
<.10\end{array}$ & $\begin{array}{l}-. \\
0.22 \\
.23 \\
.23 \\
.26\end{array}$ & $\begin{array}{l}<- \\
<0.10 \\
<.10 \\
<.10 \\
<.10\end{array}$ & $\begin{array}{r}-.1 \\
<0.10 \\
<.10 \\
.10 \\
<.10\end{array}$ & $\begin{array}{l}<-. \\
<0.10 \\
<.10 \\
<.10 \\
<.10\end{array}$ & $\begin{array}{l}-- \\
<0.10 \\
<.11 \\
.23 \\
<.10\end{array}$ & $\begin{array}{l}<- \\
<0.10 \\
<.10 \\
<.10 \\
<.10\end{array}$ \\
\hline $\begin{array}{l}\mathrm{RS} 1 \\
\mathrm{RC} 13 \\
\mathrm{RC26} \\
\mathrm{RC25} \\
\mathrm{RC} 21\end{array}$ & $\begin{array}{l}5-30-90 \\
5-30-90 \\
5-30-90 \\
5-30-90 \\
5-30-90\end{array}$ & $\begin{array}{l}1100 \\
1305 \\
0820 \\
1600\end{array}$ & 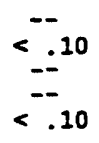 & $\begin{array}{l}-- \\
.30 \\
=- \\
.19\end{array}$ & $\begin{array}{l}<. \\
<-10 \\
<-.10\end{array}$ & $\begin{array}{l}<-.10 \\
\angle-- \\
<.10\end{array}$ & $\begin{array}{l}--.10 \\
--- \\
<.10\end{array}$ & $\begin{array}{l}<-.10 \\
--- \\
<.10\end{array}$ & $\begin{array}{l}--.10 \\
\angle-. \\
<.10\end{array}$ \\
\hline $\begin{array}{l}\mathrm{RC} 20 \\
\mathrm{RC} 11 \\
\mathrm{RC} 10 \\
\mathrm{RC15} \\
\mathrm{DC2}\end{array}$ & $\begin{array}{l}5-30-90 \\
5-30-90 \\
5-30-90 \\
5-30-90 \\
5-30-90\end{array}$ & $\begin{array}{l}1500 \\
1655 \\
1615 \\
1635 \\
1515\end{array}$ & $\begin{array}{l}<.10 \\
<.10 \\
<.10 \\
<.10\end{array}$ & $\begin{array}{l}.24 \\
.29 \\
.34 \\
.69 \\
-.\end{array}$ & $\begin{array}{l}<.10 \\
<.10 \\
<.10 \\
<.10\end{array}$ & $\begin{array}{l}<.10 \\
<.10 \\
<.16 \\
.10\end{array}$ & $\begin{array}{l}<.10 \\
<.10 \\
<.10 \\
<.10\end{array}$ & $\begin{array}{l}<.10 \\
<.10 \\
<.10 \\
<.10\end{array}$ & $\begin{array}{l}<.10 \\
<.10 \\
<.10 \\
<.10\end{array}$ \\
\hline $\begin{array}{l}\text { F45 } \\
\text { RC29 } \\
\text { RC16 } \\
\text { RC17 } \\
\text { SC10 }\end{array}$ & $\begin{array}{l}5-30-90 \\
5-30-90 \\
5-30-90 \\
5-30-90 \\
5-30-90\end{array}$ & $\begin{array}{l}1255 \\
1130 \\
0950 \\
0800 \\
0805\end{array}$ & $\begin{array}{l}<.10 \\
<.10 \\
<.10 \\
<.10 \\
<.10\end{array}$ & $\begin{array}{r}.71 \\
<.10 \\
.30 \\
.49 \\
.98\end{array}$ & $\begin{array}{l}<.10 \\
<.10 \\
<.10 \\
<.10 \\
<.10\end{array}$ & $\begin{array}{r}.10 \\
<.10 \\
.10 \\
.14 \\
.19\end{array}$ & $\begin{array}{r}.22 \\
<.10 \\
.10 \\
.10 \\
<.10\end{array}$ & $\begin{array}{l}<.10 \\
<.10 \\
<.10 \\
<.10 \\
<.10\end{array}$ & $\begin{array}{l}<.10 \\
<.10 \\
<.10 \\
<.10 \\
<.10\end{array}$ \\
\hline $\begin{array}{l}\text { SC13 } \\
\text { SC3 } \\
\text { SC6 } \\
\text { SCU1 } \\
\text { SCU2 }\end{array}$ & $\begin{array}{l}5-30-90 \\
5-30-90 \\
5-30-90 \\
5-30-90 \\
5-30-90\end{array}$ & $\begin{array}{l}0720 \\
1030 \\
1600 \\
1250 \\
1230\end{array}$ & $\begin{array}{r}.27 \\
.34 \\
.23 \\
<.10 \\
-.-\end{array}$ & $\begin{array}{l}.11 \\
.36 \\
.38 \\
.39 \\
-.\end{array}$ & $\begin{array}{l}<.10 \\
<.10 \\
<.10 \\
<.10\end{array}$ & $\begin{array}{l}<.10 \\
<.10 \\
<.10 \\
<.10 \\
--\end{array}$ & $\begin{array}{l}<.10 \\
<.10 \\
1.3 \\
<.10 \\
-\end{array}$ & $\begin{array}{l}<.10 \\
<.10 \\
<.10 \\
<.10 \\
--\end{array}$ & $\begin{array}{l}<.10 \\
<.10 \\
<.10 \\
<.10\end{array}$ \\
\hline $\begin{array}{l}\text { L23S } \\
\text { SC5 } \\
\text { SC1 } \\
\text { SC14 } \\
\text { SC2 }\end{array}$ & $\begin{array}{l}5-30-90 \\
5-30-90 \\
5-30-90 \\
5-30-90 \\
5-29-90\end{array}$ & $\begin{array}{l}1420 \\
1300 \\
1200 \\
0955 \\
1715\end{array}$ & $\begin{array}{l}<.10 \\
<.10 \\
<-.10 \\
<.10\end{array}$ & $\begin{array}{l}.37 \\
.33 \\
-. \\
.33 \\
.19\end{array}$ & $\begin{array}{l}<.10 \\
<.10 \\
<.10 \\
<.10\end{array}$ & $\begin{array}{l}<.10 \\
<.10 \\
<.10 \\
<.10\end{array}$ & $\begin{array}{l}<.10 \\
<.10 \\
-.10 \\
<.10\end{array}$ & $\begin{array}{l}<.10 \\
<.10 \\
-.10 \\
<.10\end{array}$ & $\begin{array}{l}<.10 \\
<.10 \\
-.1 \\
<.10 \\
<.10\end{array}$ \\
\hline $\begin{array}{l}\text { SC4 } \\
\text { RC18 } \\
\text { RC19 } \\
\text { F47 } \\
\text { RC24 }\end{array}$ & $\begin{array}{l}5-29-90 \\
5-30-90 \\
5-30-90 \\
5-30-90 \\
5-30-90\end{array}$ & $\begin{array}{l}1550 \\
0755 \\
0945 \\
1135 \\
1325\end{array}$ & $\begin{array}{r}.68 \\
.22 \\
<.10 \\
.18 \\
.16\end{array}$ & $\begin{array}{l}1.0 \\
.54 \\
.43 \\
.47 \\
.48\end{array}$ & $\begin{array}{l}<.10 \\
<.10 \\
<.10 \\
<.10 \\
<.10\end{array}$ & $\begin{array}{r}<.10 \\
.14 \\
.20 \\
.21 \\
.21\end{array}$ & $\begin{array}{r}.14 \\
<.10 \\
.12 \\
.12 \\
<.10\end{array}$ & $\begin{array}{l}<.10 \\
<.10 \\
<.10 \\
<.10 \\
<.10\end{array}$ & $\begin{array}{l}<.10 \\
<.10 \\
<.10 \\
<.10 \\
<.10\end{array}$ \\
\hline $\begin{array}{l}\mathrm{RC22} \\
\mathrm{RC2} \\
\mathrm{BC1} \\
\mathrm{BC2}\end{array}$ & $\begin{array}{l}5-30-90 \\
5-30-90 \\
5-29-90 \\
5-29-90\end{array}$ & $\begin{array}{l}1515 \\
1655 \\
1520 \\
1710\end{array}$ & $\begin{array}{r}.12 \\
.10 \\
<.10 \\
.13\end{array}$ & $\begin{array}{l}.60 \\
.52 \\
.13 \\
.45\end{array}$ & $\begin{array}{l}<.10 \\
<.10 \\
<.10 \\
<.10\end{array}$ & $\begin{array}{r}.21 \\
\times .17 \\
<.10 \\
<.10\end{array}$ & $\begin{array}{l}<.10 \\
<.10 \\
<.10 \\
<.10\end{array}$ & $\begin{array}{l}<.10 \\
<.10 \\
<.10 \\
<.10\end{array}$ & $\begin{array}{l}<.10 \\
<.10 \\
<.10 \\
<.10\end{array}$ \\
\hline
\end{tabular}


Table 32. Onsite determinations, nitrogen species, and suspended sediment in a selected reach of Roberts Creek, Clayton County, Iowa, April-November 1990

$\left[\mathrm{ft}^{3} / \mathrm{s}\right.$, cubic feet per second; ${ }^{\circ} \mathrm{C}$, degrees Celsius; $\mu \mathrm{S} / \mathrm{cm}$, microsiemens per centimeter at 25 degrees Celsius; nitrogen species and suspended-sediment concentrations in milligrams per liter; $<$, less than detection level indicated; --, missing data]

\begin{tabular}{|c|c|c|c|c|c|c|c|c|}
\hline Date & Time & $\begin{array}{l}\text { Instan- } \\
\text { taneous } \\
\text { dis- } \\
\text { chafge } \\
\left(\mathrm{ft}^{3} / \mathrm{s}\right)\end{array}$ & $\begin{array}{l}\text { Temper- } \\
\text { ature } \\
\left({ }^{\circ} \mathrm{C}\right)\end{array}$ & $\begin{array}{l}\text { Spe- } \\
\text { cific } \\
\text { con- } \\
\text { duct- } \\
\text { ance pH } \\
(\mu S / \mathrm{cm})(\text { units }\end{array}$ & $\begin{array}{l}\text { Total } \\
\text { nitrite } \\
\text { plus } \\
\text { nitrate } \\
\text { ) (as N) }\end{array}$ & $\begin{array}{l}\text { Total } \\
\text { ammonia } \\
\text { (as N) }\end{array}$ & $\begin{array}{l}\text { Total } \\
\text { organic } \\
\text { nitrógen } \\
\quad(\text { as } N)\end{array}$ & $\begin{array}{l}\text { Sus- } \\
\text { pended } \\
\text { sedi- } \\
\text { ment }\end{array}$ \\
\hline
\end{tabular}

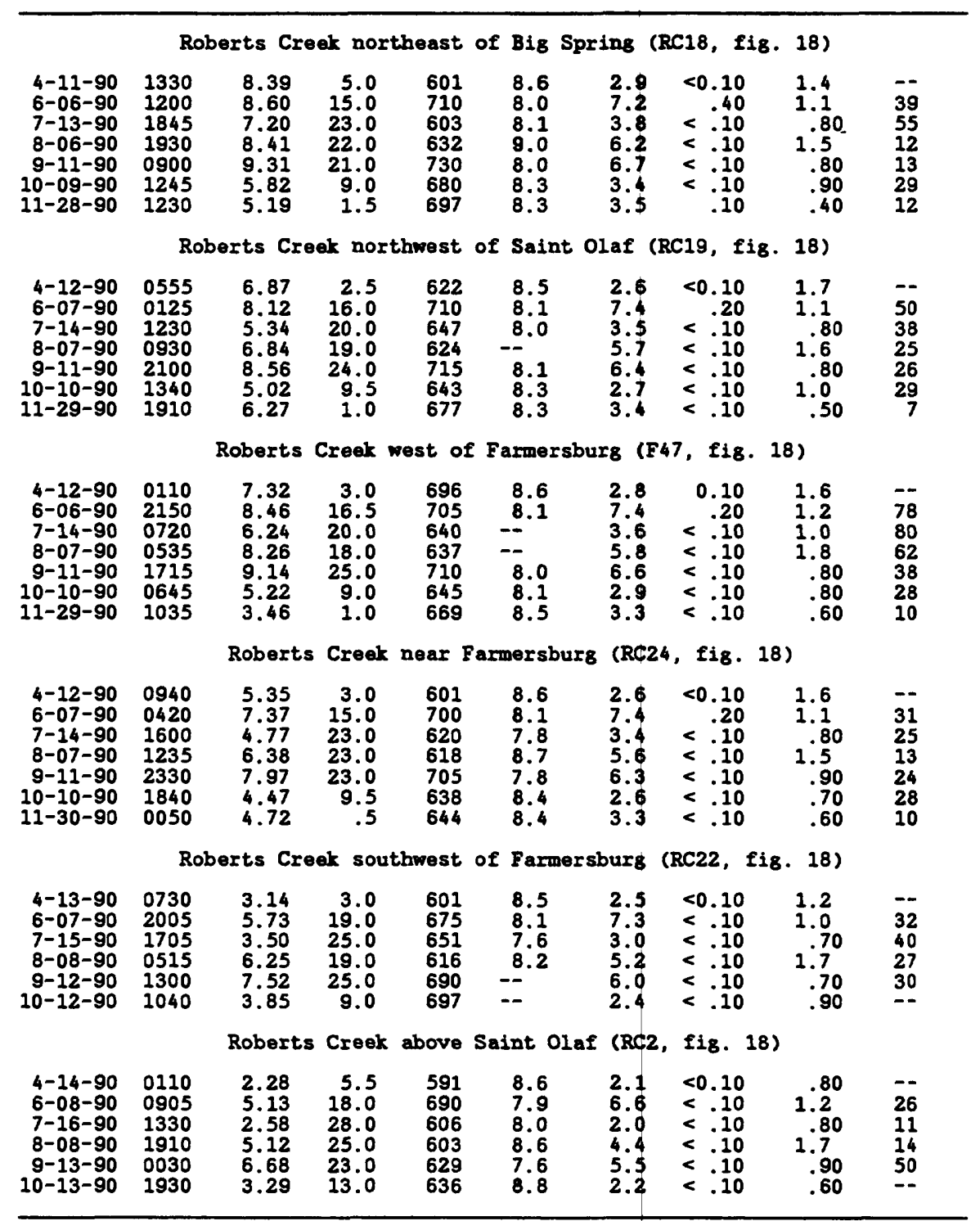


Table 33. Herbicide and herbicide metabolite concentrations in a selected reach of Roberts Creek, Clayton County, Iowa, April-November 1990

[Total-recoverable constituents in micrograms per liter; DDAR, deisopropylatrazine plus deethylatrazine-to-atrazine ratio; $<$, less than detection level indicated]

\begin{tabular}{|c|c|c|c|c|c|c|c|}
\hline Date & $\begin{array}{c}\text { Time } \\
\text { (24-hour) }\end{array}$ & $\begin{array}{l}\text { Ala- } \\
\text { chlor }\end{array}$ & $\begin{array}{r}\text { Atra- } \\
\text { zine }\end{array}$ & $\begin{array}{l}\text { Cyana- } \\
\text { ane }\end{array}$ & DDAR & $\begin{array}{l}\text { Deiso- } \\
\text { propy 1- } \\
\text { atra- } \\
\text { zine }\end{array}$ & $\begin{array}{l}\text { Deethyl- Metola- } \\
\text { atra- chlor } \\
\text { zine }\end{array}$ \\
\hline
\end{tabular}

\begin{tabular}{rccccccccc}
\hline \multicolumn{1}{c}{ Roberts Creek northeast of B18 Spring } & (RC18, 118. 18)- \\
$4-11-90$ & 1330 & 0.11 & 0.68 & $<0.20$ & 0.27 & $<0.05$ & 0.15 & $<0.05$ \\
$6-06-90$ & 1200 & 2.3 & 4.8 & 3.7 & .25 & .34 & .67 & 1.0 \\
$7-13-90$ & 1845 & $<.05$ & .37 & $<.20$ & .69 & $<.05$ & .21 & .05 \\
$8-06-90$ & 1930 & .06 & 2.49 & $<.20$ & .48 & .25 & .77 & .15 \\
$9-11-90$ & 0900 & .06 & .24 & $<.20$ & 1.4 & .08 & .21 & $<.05$ \\
$10-09-90$ & 1245 & .19 & .40 & $<.20$ & .59 & .06 & .14 & .11 \\
$11-28-90$ & 1230 & $<.05$ & .09 & $<.20$ & 1.4 & $<.05$ & .10 & $<.05$
\end{tabular}

Roberts Creek northwest of Saint Olaf (RC19, fig. 18)

$\begin{array}{rrrrrrrrrr}4-12-90 & 0110 & 0.12 & 0.60 & <0.20 & 0.45 & 0.07 & 0.16 & 0.05 \\ 6-06-90 & 2150 & 3.0 & 5.8 & 2.9 & .23 & .37 & .73 & .97 \\ 7-14-90 & 0720 & <.05 & .41 & <.20 & .59 & <.05 & .20 & 0.05 \\ 8-07-90 & 0535 & .05 & 2.6 & <.20 & .48 & .25 & .82 & .17 \\ 9-11-90 & 1715 & .05 & .21 & <.20 & 1.6 & .08 & .20 & <.05 \\ 10-10-90 & 0645 & .14 & .28 & <.20 & .76 & .05 & .13 & .09 \\ 11-29-90 & 1035 & <.05 & .08 & <.20 & 1.6 & <.05 & .05 & .05\end{array}$

Roberts Creek west of Farmersburg (F47, fig. 18)

$\begin{array}{rrrrrrrrr}4-12-90 & 0555 & 0.11 & 0.54 & <0.20 & 0.48 & 0.05 & 0.17 & 0.05 \\ 6-07-90 & 0125 & 3.2 & 6.3 & 2.5 & .22 & .37 & .79 & 1.1 \\ 7-14-90 & 1230 & <.05 & .37 & <.20 & .62 & <.05 & .19 & <.05 \\ 8-07-90 & 0930 & .06 & 2.3 & <.20 & .49 & .21 & .76 & .17 \\ 9-11-90 & 2100 & .05 & .22 & <.20 & 1.4 & .06 & .21 & 0.05 \\ 10-10-90 & 1340 & .14 & .25 & <.20 & .69 & <.05 & .14 & .07 \\ 11-29-90 & 1910 & <.05 & .10 & <.20 & 1.6 & <.05 & .13 & .05\end{array}$

Roberts Creek near Farmersburg (RC24, f18. 18)

$\begin{array}{rrrrrrrrrr}4-12-90 & 0940 & <0.05 & 0.36 & <0.20 & 0.69 & 0.06 & 0.15 & <0.05 \\ 6-07-90 & 0420 & 3.1 & 6.4 & 2.6 & .21 & .36 & .76 & 1.0 \\ 7-14-90 & 1600 & <.05 & .39 & <.20 & .59 & <.05 & .19 & <.05 \\ 8-07-90 & 1235 & & .05 & 2.2 & <.20 & .49 & .22 & .70 & .16 \\ 9-11-90 & 2330 & <.05 & .21 & <.20 & 1.4 & .06 & .19 & 0.05 \\ 10-10-90 & 1840 & & .12 & .21 & <.20 & .72 & <.05 & .12 & .07 \\ 11-30-90 & 0050 & <.05 & .09 & <.20 & 1.5 & <.05 & .11 & .05\end{array}$

Roberts Creek southwest of Farmersburg (RC22, fig. 18)

\begin{tabular}{|c|c|c|c|c|c|c|c|c|}
\hline $\begin{array}{r}4-13-90 \\
6-07-90 \\
7-15-90 \\
8-08-90 \\
9-12-90 \\
10-12-90\end{array}$ & $\begin{array}{l}0730 \\
2005 \\
1705 \\
0515 \\
1300 \\
1040\end{array}$ & $\begin{array}{r}0.12 \\
3.4 \\
<\quad .05 \\
.05 \\
<.05 \\
.15\end{array}$ & $\begin{array}{l}0.48 \\
9.0 \\
.15 \\
1.8 \\
.21 \\
.30\end{array}$ & $\begin{array}{l}<0.20 \\
1.8 \\
<\quad .20 \\
<.20 \\
<.20 \\
<.20\end{array}$ & $\begin{array}{r}0.61 \\
.20 \\
.62 \\
.56 \\
1.4 \\
.90\end{array}$ & $\begin{array}{r}0.07 \\
<.45 \\
.05 \\
.19 \\
.06 \\
.06\end{array}$ & $\begin{array}{l}0.18 \\
1.0 \\
.07 \\
.69 \\
.19 \\
.17\end{array}$ & $\begin{array}{r}0.05 \\
1.2 \\
<\quad .05 \\
.20 \\
<.05 \\
.08\end{array}$ \\
\hline & \multicolumn{8}{|c|}{ Roberts Creek above Saint Olaf (RC2, fig. 18) } \\
\hline $\begin{array}{r}4-14-90 \\
6-08-90 \\
7-16-90 \\
8-08-90 \\
9-13-90 \\
10-13-90\end{array}$ & $\begin{array}{l}0110 \\
0905 \\
1330 \\
1910 \\
0030 \\
1930\end{array}$ & $\begin{array}{l}0.06 \\
3.6 \\
<\quad .05 \\
<.05 \\
.06 \\
.12\end{array}$ & $\begin{array}{r}0.37 \\
17 \\
.46 \\
.95 \\
.19 \\
.29\end{array}$ & $\begin{array}{l}<0.20 \\
2.5 \\
<\quad .20 \\
<.20 \\
<.20 \\
<\quad .20\end{array}$ & $\begin{array}{r}0.70 \\
.08 \\
.55 \\
.50 \\
1.7 \\
.85\end{array}$ & $\begin{array}{r}0.06 \\
1.05 \\
<\quad .05 \\
.31 \\
.18 \\
.06\end{array}$ & $\begin{array}{l}0.16 \\
.05 \\
.21 \\
.11 \\
.08 \\
.15\end{array}$ & $\begin{array}{r}<0.05 \\
1.4 \\
<\quad .05 \\
.09 \\
.06 \\
.08\end{array}$ \\
\hline
\end{tabular}


Table 34. Herbicide concentrations in a selected reach of Roberts Creek, Clayton County, Iowa, April-November 1990

[Concentrations in micrograms per liter; <, less than detection level indicated]

Date $\begin{gathered}\text { Time Ametryn Prometon } \\ (24-\text { hour })\end{gathered}$

Roberts Creek northeast of Big Spring (RC18, fig. 18)

\begin{tabular}{|c|c|c|c|c|c|c|c|}
\hline $\begin{array}{r}4-11-90 \\
6-06-90 \\
7-13-90 \\
8-06-90 \\
9-11-90 \\
10-09-90 \\
11-28-90\end{array}$ & $\begin{array}{l}1330 \\
1200 \\
1845 \\
1930 \\
0900 \\
1245 \\
1230\end{array}$ & $\begin{array}{l}<0.05 \\
<.05 \\
<.05 \\
<.05 \\
<.05 \\
<.05 \\
<.05\end{array}$ & $\begin{array}{l}<0.05 \\
<.05 \\
<.05 \\
<.05 \\
<.05 \\
<.05 \\
<.05\end{array}$ & $\begin{array}{l}<0.05 \\
<.05 \\
<.05 \\
<.05 \\
<.05 \\
<.05 \\
<.05\end{array}$ & $\begin{array}{l}<0.05 \\
<.09 \\
<.05 \\
<.05 \\
<.05 \\
<.05 \\
<.05\end{array}$ & $\begin{array}{l}<0.05 \\
<.22 \\
<.05 \\
<.05 \\
<.05 \\
<.05 \\
<.05\end{array}$ & $\begin{array}{l}<0.05 \\
<.05 \\
<.05 \\
<.05 \\
<.05 \\
<.05 \\
<.05\end{array}$ \\
\hline
\end{tabular}

Roberts Creek northwest of Saint Olaf (RC19, fig. 18)

\begin{tabular}{|c|c|c|c|c|c|c|c|}
\hline $\begin{array}{r}4-12-90 \\
6-07-90 \\
7-14-90 \\
8-07-90 \\
9-11-90 \\
10-10-90 \\
11-29-90\end{array}$ & $\begin{array}{l}0555 \\
0125 \\
1230 \\
0930 \\
2100 \\
1340 \\
1910\end{array}$ & $\begin{array}{l}<0.05 \\
<.05 \\
<.05 \\
<.05 \\
<.05 \\
<.05 \\
<.05\end{array}$ & $\begin{array}{l}<0.05 \\
<\quad .05 \\
<.05 \\
<.05 \\
<.05 \\
<.06 \\
<.05\end{array}$ & $\begin{array}{l}<0.05 \\
<\quad .05 \\
<.05 \\
<.05 \\
<.05 \\
<.05 \\
<.05\end{array}$ & $\begin{array}{r}<0.05 \\
<.05 \\
<.05 \\
<.05 \\
<.05 \\
<.05\end{array}$ & $\begin{array}{r}<0.05 \\
<.34 \\
<.05 \\
<.05 \\
<.05 \\
<.05\end{array}$ & $\begin{array}{l}<0.05 \\
<\quad .05 \\
<.05 \\
<.05 \\
<.05 \\
<.05 \\
<.05\end{array}$ \\
\hline & Robe & \multicolumn{6}{|l|}{ Creek } \\
\hline $\begin{array}{r}4-12-90 \\
6-06-90 \\
7-14-90 \\
8-07-90 \\
9-11-90 \\
10-10-90\end{array}$ & $\begin{array}{l}0110 \\
2150 \\
0720 \\
0535 \\
1715 \\
0645 \\
1035\end{array}$ & $\begin{array}{l}<0.05 \\
<\quad .05 \\
<.05 \\
<.05 \\
<.05 \\
<.05 \\
<.05\end{array}$ & $\begin{array}{l}<0.05 \\
<.05 \\
<.05 \\
<.05 \\
<.05 \\
<.05 \\
<.05\end{array}$ & $\begin{array}{l}<0.05 \\
<\quad .05 \\
<.05 \\
<.05 \\
<.05 \\
<.05 \\
<.05\end{array}$ & $\begin{array}{r}<0.05 \\
<.11 \\
<.05 \\
<.05 \\
<.05 \\
<.05\end{array}$ & $\begin{array}{r}<0.05 \\
<.09 \\
<.05 \\
<.05 \\
<.05 \\
<.05\end{array}$ & $\begin{array}{l}<0.05 \\
<.05 \\
<.05 \\
<.05 \\
<.05 \\
<.05 \\
<.05\end{array}$ \\
\hline & \multicolumn{7}{|c|}{ Roberts } \\
\hline $\begin{array}{r}4-12-90 \\
6-07-90 \\
7-14-90 \\
8-07-90 \\
9-11-90 \\
10-10-90 \\
11-30-90\end{array}$ & $\begin{array}{l}0940 \\
0420 \\
1600 \\
1235 \\
2330 \\
1840 \\
0050\end{array}$ & $\begin{array}{l}<0.05 \\
<.05 \\
<.05 \\
<.05 \\
<.05 \\
<.05 \\
<.05\end{array}$ & $\begin{array}{l}<0.05 \\
<\quad .05 \\
<\quad .05 \\
<.05 \\
<.05 \\
<.05 \\
<.05\end{array}$ & $\begin{array}{l}<0.05 \\
<\quad .05 \\
<\quad .05 \\
<.05 \\
<.05 \\
<.05 \\
<\quad .05\end{array}$ & $\begin{array}{r}<0.05 \\
<.13 \\
<.05 \\
<.05 \\
<.05 \\
<.05 \\
<.05\end{array}$ & $\begin{array}{r}<0.05 \\
<.34 \\
<.05 \\
<.05 \\
<.05 \\
<.05\end{array}$ & $\begin{array}{l}<0.05 \\
<.05 \\
<.05 \\
<.05 \\
<.05 \\
<.05 \\
<.05\end{array}$ \\
\hline
\end{tabular}

Roberts Creek southwest of Farmersburg (RC22, fig. 18)

\begin{tabular}{|c|c|c|c|c|c|c|c|}
\hline $\begin{array}{r}4-13-90 \\
6-07-90 \\
7-15-90 \\
8-08-90 \\
9-12-90 \\
10-12-90\end{array}$ & $\begin{array}{l}0730 \\
2005 \\
1705 \\
0515 \\
1300 \\
1040\end{array}$ & $\begin{array}{l}<0.05 \\
<.05 \\
<.05 \\
<.05 \\
<.05 \\
<.05\end{array}$ & $\begin{array}{l}<0.05 \\
<.05 \\
<.05 \\
<.05 \\
<.05 \\
.06\end{array}$ & $\begin{array}{l}<0.05 \\
<.05 \\
<.05 \\
<.05 \\
<.05 \\
<.05\end{array}$ & $\begin{array}{r}<0.05 \\
<.19 \\
<.05 \\
<.05 \\
<.05 \\
<.05\end{array}$ & $\begin{array}{l}<0.05 \\
<.48 \\
<.05 \\
<.05 \\
<.05 \\
<.05\end{array}$ & $\begin{array}{l}<0.05 \\
<.05 \\
<.05 \\
<.05 \\
<.05 \\
<.05\end{array}$ \\
\hline \multicolumn{8}{|c|}{ Roberts Creek above Saint olaf (RC2, fig, 18) } \\
\hline $\begin{array}{r}4-14-90 \\
6-08-90 \\
7-16-90 \\
8-08-90 \\
9-13-90 \\
10-13-90\end{array}$ & $\begin{array}{l}0110 \\
0905 \\
1330 \\
1910 \\
0030 \\
1930\end{array}$ & $\begin{array}{l}<0.05 \\
<.05 \\
<.05 \\
<.05 \\
<.05 \\
<.05\end{array}$ & $\begin{array}{l}<0.05 \\
<.05 \\
<.05 \\
<.05 \\
<.05 \\
<.05\end{array}$ & $\begin{array}{l}<0.05 \\
<.05 \\
<.05 \\
<.05 \\
<.05 \\
<.05\end{array}$ & $\begin{array}{r}<0.05 \\
<.22 \\
<.05 \\
<.05 \\
<.05 \\
<.05\end{array}$ & $\begin{array}{l}<0.05 \\
<.48 \\
<.05 \\
<.05 \\
<.05 \\
<.05\end{array}$ & $\begin{array}{l}<0.05 \\
<.05 \\
<.05 \\
<.05 \\
<.05 \\
<.05\end{array}$ \\
\hline
\end{tabular}

